\title{
Adipose tissue metabolism and cardiometabolic health in obesity
}

Citation for published version (APA):

Stinkens, R. M. E. (2017). Adipose tissue metabolism and cardiometabolic health in obesity: effects of pharmacological and lifestyle interventions. [Doctoral Thesis, Maastricht University]. Maastricht University. https://doi.org/10.26481/dis.20171005rs

Document status and date:

Published: 01/01/2017

DOI:

10.26481/dis.20171005rs

Document Version:

Publisher's PDF, also known as Version of record

\section{Please check the document version of this publication:}

- A submitted manuscript is the version of the article upon submission and before peer-review. There can be important differences between the submitted version and the official published version of record.

People interested in the research are advised to contact the author for the final version of the publication, or visit the DOI to the publisher's website.

- The final author version and the galley proof are versions of the publication after peer review.

- The final published version features the final layout of the paper including the volume, issue and page numbers.

Link to publication

\footnotetext{
General rights rights.

- You may freely distribute the URL identifying the publication in the public portal. please follow below link for the End User Agreement:

www.umlib.nl/taverne-license

Take down policy

If you believe that this document breaches copyright please contact us at:

repository@maastrichtuniversity.nl

providing details and we will investigate your claim.
}

Copyright and moral rights for the publications made accessible in the public portal are retained by the authors and/or other copyright owners and it is a condition of accessing publications that users recognise and abide by the legal requirements associated with these

- Users may download and print one copy of any publication from the public portal for the purpose of private study or research.

- You may not further distribute the material or use it for any profit-making activity or commercial gain

If the publication is distributed under the terms of Article $25 \mathrm{fa}$ of the Dutch Copyright Act, indicated by the "Taverne" license above, 


\section{Adipose tissue metabolism and cardiometabolic health in obesity}

Effects of pharmacological and lifestyle interventions 
The research described in this thesis was performed within NUTRIM School of Nutrition and Translational Research in Metabolism and was supported by Novartis Pharma AG, Unilever and by grants from the Dutch Diabetes Research Foundation (grant NL2009.60.003) and the Research Foundation Flanders (KAN 1507217N).

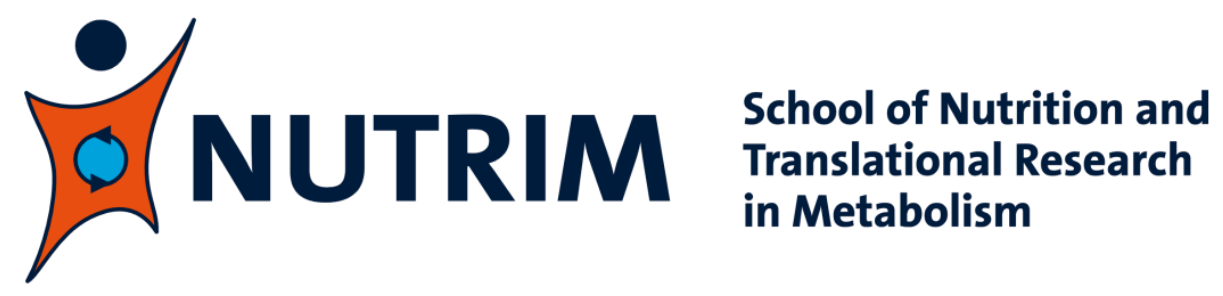

Layout \& cover design: Rudi Stinkens

Printed by: $\quad$ Gildeprint - www.gildeprint.nl

ISBN:

978-94-6233-699-5

(C) Rudi Stinkens, 2017, Maastricht, The Netherlands

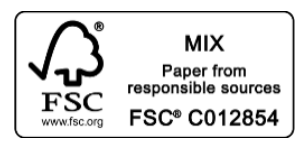

For articles published or accepted for publication, the copyright has been transferred to the respective publisher. No parts of this thesis may be reproduced, stored in a retrieval system, or transmitted in any form or by any means without the permission of the author, or, when appropriate, from the publishers of the manuscript. 


\section{Adipose tissue metabolism and cardiometabolic health in obesity}

Effects of pharmacological and lifestyle interventions

\section{PROEFSCHRIFT}

ter verkrijging van de graad van doctor aan de Universiteit Maastricht, op gezag van de Rector Magnificus, Prof. dr. Rianne M. Letschert volgens het besluit van het College van Decanen, in het openbaar te verdedigen op Donderdag 5 Oktober 2017 om 12.00 uur.

door

Rudi Michel Elisabeth Stinkens 


\section{PROMOTOR}

Prof. dr. E.E. Blaak

\section{CO-PROMOTOR}

Dr. G.H. Goossens

\section{BEOORDELINGSCOMMISSIE}

Prof. dr. C.D.A. Stehouwer (Chair)

Prof. dr. J.F.C. Glatz

Prof. dr. A.H. Kersten

(Wageningen University \& Research)

Prof. dr. L.J.C. van Loon

Prof. dr. B.M. Stallknecht

(University of Copenhagen) 
Voor papa en mama 
TABLE OF CONTENTS 
10 Chapter 1

General introduction

$38 \quad$ Chapter 2

Targeting fatty acid metabolism to improve glucose metabolism

\section{Chapter 3}

Improved insulin sensitivity with angiotensin receptor neprilysin inhibition in individuals with obesity and hypertension

\section{Chapter 4}

Effect of sacubitril/valsartan on exercise induced lipid metabolism in individuals with obesity and hypertension

162 Chapter 5

The effects of angiotensin receptor neprilysin inhibition by sacubitril/valsartan on adipose tissue transcriptome and protein expression in obese hypertensive patients

180 Chapter 6

Exercise training-induced effects on the abdominal subcutaneous adipose tissue phenotype in obese humans

202 Chapter 7

Coordinated regulation of adipose tissue adrenergicand non-adrenergic-mediated lipolysis during exercise in lean and obese individuals: the effect of exercise training

\section{Chapter 8}

General discussion

\section{Chapter 9}

Addenda 
CHAPTER 1 
GENERAL INTRODUCTION 


\section{OBESITY - THE BURDEN OF A BIG PLAYER}

Over the last decades, the worldwide prevalence of obesity has increased enormously and reached pandemic proportions. According to the World Health Organization, $39 \%$ of adults over 18 years of age (38\% of men and $40 \%$ of women) were overweight, while $13 \%$ of the world's adult population (11\% of men and $15 \%$ of women) was obese in 2014 [1]. Furthermore, globally, approximately $6.3 \%$ of the children under the age of 5 years were overweight [2] and on their way to become obese. Clearly, not only the Western society is affected by obesity, also many lowand middle-income countries have increased morbidity and mortality rates due to the increasing prevalence of obesity [1]. Nowadays, the amount of people suffering from obesity exceeds the number of people suffering from malnutrition [3], making obesity the $5^{\text {th }}$ leading cause of death worldwide [1].

Obesity is associated with an increased risk for developing insulin resistance [4] and type 2 diabetes [5]. Peripheral insulin resistance is defined as a reduced ability of insulin to stimulate glucose uptake in peripheral tissues, mainly skeletal muscle [6], while type 2 diabetes is characterized by insulin resistance accompanied with pancreatic beta-cell failure (inability of the pancreatic beta-cells to adequately secrete insulin) [7]. Furthermore, obesity is also related to the development of hypertension and cardiovascular diseases [8], fatty liver disease [9], mental disorders (e.g. depression) [10], musculoskeletal disorders (e.g. osteoarthritis) [11] and certain types of cancer $[12,13]$.

Since obesity is a major public health issue and one of the most important risk factors for the development of cardiometabolic diseases, it is needless to say that the increasing obesity prevalence has major socioeconomic consequences [3]. The World Health Organization defines overweight as a body mass index (BMI) between $25-30 \mathrm{~kg} / \mathrm{m}^{2}$ and obesity as a BMI of $30 \mathrm{~kg} / \mathrm{m}^{2}$ or greater [1]. However, the use of BMI gives an underestimation of the amount of people suffering from obesity and the related health complications globally $[14,15]$ since it does not take into account the body composition (i.e. skeletal muscle mass) and body fat distribution (i.e. abdominal and gluteofemoral fat depot) [16]. Also, the use of universal BMI cut-off points does not consistently reflect adiposity in different ethnic populations. South Asian populations, displaying a greater proportion of body fat for a given BMI than Caucasians [17], have a higher susceptibility to develop type 2 diabetes and coronary artery disease, despite lower BMI values [18]. Therefore, BMI is only an approximation and should be considered as a rough indicator to determine obesity and cardiometabolic disease risk [19]. Nowadays, more sensitive techniques are available and assessment of a patient's metabolic phenotype is needed to enhance diagnosis, prognosis and optimization of interventions [20]. Metabolic phenotyping generates detailed information regarding a patient's (patho)physiological state and makes it possible to stratify subjects into different subgroups. This stratification at baseline may improve the effectiveness of a particular intervention in a specific subgroup of the population [21]. Therefore, detailed metabolic phenotyping is necessary to identify individuals or subgroups of a population that have an increased risk for developing metabolic diseases and to optimize prevention and treatment strategies. 


\section{OBESITY AND CARDIOMETABOLIC HEALTH}

The etiology of obesity is complex and multifactorial. However, the fundamental cause of overweight and obesity is an imbalance between energy intake (i.e. energy-dense diet) and energy expenditure (i.e. physical inactivity), which depends on the interaction between biological, genetic, behavioral, social and environmental factors [22].

The development of obesity is accompanied by a substantial increase in adipose tissue mass. While adipose tissue used to be considered a passive organ, only involved in the storage and release of energy, it has become clear over the last two decades that adipose tissue is an active metabolic and endocrine organ. Moreover, adipose tissue mass per se may not be the most important contributor to the development of obesity-related disorders. For example, abdominal liposuction, which is the surgical removal of abdominal subcutaneous adipose tissue, did not significantly improve obesity-related disturbances like insulin resistance [23]. Furthermore, pharmacological treatment with insulin-sensitizing thiazolidinediones (PPARy agonists) is accompanied by a significant increase in subcutaneous adipose tissue mass [24]. Additionally, patients with lipodystrophy, who are characterized by a partial or complete lack of adipose tissue, have pronounced insulin resistance and a high incidence of type 2 diabetes [25]. Collectively, these findings indicate that total adipose tissue mass per se is not the predominant contributor to the development of obesity-related complications. Body fat distribution and adipose tissue (dys)function seem to play a more prominent role in cardiometabolic health [26, 27]. Adipose tissue accumulation in the upper body (abdominal region) is associated with the development of obesity-related comorbidities and all-cause mortality, while fat accumulation in the lower body (gluteofemoral region) is considered to be even protective against metabolic and cardiovascular disturbances $[28,29]$. Apparently, the functional properties of these adipose tissue depots, seem to play an important role in the disease risk [16]. Adipose tissue dysfunction, which is characterized by an impaired capacity to store lipids in combination with low-grade inflammation, seems to contribute to the development of insulin resistance and impaired glucose metabolism by promoting excessive fat storage in non-adipose tissues (ectopic fat deposition), such as the liver, skeletal muscle, pancreas and heart [26, 30-33]. Furthermore, functional impairments in either of these organs may further contribute to the development of impaired glucose metabolism, type 2 diabetes and cardiovascular disease, as extensively described in chapter 2 of this thesis [34].

Interestingly, around $10-30 \%$ of the obese population seems to be relatively protected against the development of cardiometabolic complications and are therefore referred to as 'metabolically healthy obese' (MHO) $[35,36]$. The reason why these obese individuals are protected from cardiometabolic complications is currently not completely understood, but may be caused by several mechanisms, including a beneficial body fat distribution (low visceral and ectopic fat storage compared to subcutaneous fat depots), normal adipose tissue function (a normal adipose tissue inflammatory phenotype and adipokine secretion pattern) as well as high physical activity and fitness levels, together contributing to preserved insulin sensitivity [37-39]. Importantly, at a given BMI, there are obese individuals who are either very insulin sensitive or extremely insulin resistant. Thus, there is no clear 
BMI-cutoff separating these distinct obese subgroups [35]. It is also important to acknowledge that the criteria to define $\mathrm{MHO}$ have only been established recently $[36,40]$ and studies performed thus far characterized $\mathrm{MHO}$ as the absence of metabolic disorders, such as dyslipidemia, insulin resistance, impaired glucose metabolism and type 2 diabetes. Interestingly, the concept of $\mathrm{MHO}$ may be misleading, since several meta-analyses of prospective cohort studies showed an increased risk of developing type 2 diabetes [41] and cardiovascular disease [42, 43] over time when compared to healthy normal weight subjects. The duration of a $\mathrm{MHO}$ phenotype for 5.5-10.3 years of follow-up was not associated with an increased risk to develop diabetes and cardiovascular disease, compared to metabolically healthy normal weight subjects, but in about one-third of the $\mathrm{MHO}$ subjects, the healthy phenotype was transient [44]. However, a $24 \%$ increased mortality and cardiovascular risk was observed in the $\mathrm{MHO}$ group compared to healthy normal weight subjects, when the MHO phenotype persisted over 10 years of follow up [42]. Clearly, the $\mathrm{MHO}$ individuals may develop a metabolically 'unhealthy' phenotype $[45,46]$ over time and therefore $\mathrm{MHO}$ should not be considered a harmless condition [47, 48]. Major efforts should be made to prevent obesity and obesity-related comorbidities to maintain or reach a metabolically healthy phenotype.

There is substantial evidence to suggest that targeting the renin-angiotensin system and the natriuretic peptide system, either via pharmacological treatment or lifestyle intervention, may beneficially affect cardiometabolic health. In the next paragraphs, these two systems and their metabolic effects will be described in more detail. Thereafter, the potential of physical exercise interventions to enhance metabolic health will be addressed.

\section{THE RENIN-ANGIOTENSIN SYSTEM}

The renin-angiotensin system (RAS) is known as an important regulator of systemic blood pressure and electrolyte homeostasis [49]. It has also been shown that several RAS components are increased in obesity and insulin resistance and are present in different tissues, including the adipose tissue [50-53]. Furthermore, an increased RAS activity has been linked to the development of type 2 diabetes and cardiovascular disease [54, 55].

In the classical (systemic) RAS, renal-derived renin converts circulating angiotensinogen (AGT), mainly produced in the liver, into angiotensin I (ANG I), which is then converted to angiotensin II (ANG II) by the action of angiotensinconverting enzyme (ACE) found in the lung capillaries. ANG II is the main effector hormone of the RAS and binds to the ANG II type $1\left(\mathrm{AT}_{1}\right)$ and ANG II type $2\left(\mathrm{AT}_{2}\right)$ receptors to exert its biological functions. Most of the (patho)physiological effects, such as vasoconstriction, aldosterone production, sodium reabsorption and nervous system activation are induced by binding of ANG II to AT ${ }_{1}$. In contrast, binding of $A N G$ II to $A T_{2}$ counteracts the $A T_{1}$-mediated effects and promotes vasodilation, natriuresis and anti-inflammatory responses. Clearly, $A T_{1}$ and $A T_{2}$ receptors induce opposite physiological and metabolic effects [54]. 


\section{Metabolic effects of the renin-angiotensin system}

Different RAS components have been identified in several tissues, such as the adipose tissue [50,51], skeletal muscle and pancreas [52, 53] and a role for the local RAS in obesity-related metabolic disturbances has been suggested [54, 56]. The metabolic effects of RAS on adipose tissue, skeletal muscle and pancreatic function or metabolism will be addressed below.

\section{The renin-angiotensin system and adipose tissue function}

The adipose tissue of obese, insulin resistant and type 2 diabetic patients is characterized by enlarged (hypertrophic) adipocytes, which can be attributable to a prolonged positive energy balance and impaired adipocyte differentiation. On the one hand, ANG II has been shown to promote adipocyte growth and differentiation via several mechanisms [57,58], but on the other hand it has also been shown to inhibit differentiation of cultured human preadipocytes, mediated via the $\mathrm{AT}_{1}$ receptor [59] and to reduce insulin-induced adipocyte differentiation in human preadipocytes [60]. Long-term RAS blockade, with the angiotensin receptor blocker (ARB) valsartan, reduced abdominal subcutaneous adipocyte size and increased the amount of small adipocytes in obese individuals with impaired glucose metabolism [61].

Furthermore, ANG II has been shown to increase lipid synthesis and storage in both rodent and human adipocytes [58] and to reduce adipose tissue lipolysis [6264], while blockade of the RAS reversed these effects [65]. However, conflicting results have also been reported, with ANG II increasing adipose tissue lipolysis in lean, but not in obese men [66] and RAS blockade not affecting adipose tissue lipolysis in obese subjects [67].

Adipocyte size plays an important role in adipokine expression and secretion, with a more pro-inflammatory phenotype of hypertrophic adipocytes. It has been shown that ANG II increased, while RAS blockade decreased adipose tissue gene expression of pro-inflammatory markers in rodents, as reviewed [50, 51]. In human adipose tissue, long-term RAS blockade with valsartan reduced the expression of macrophage infiltration markers [61] and several studies observed that ARB treatment altered circulating adipokine concentrations, although conflicting results have been reported [50].

Another important contributor to adipose tissue lipid handling is adipose tissue blood flow (ATBF), which is reduced in obese, insulin resistant and type 2 diabetic individuals, in both the fasting and postprandial state, as reviewed elsewhere [68]. While ANG II reduces ATBF [62, 69], local RAS blockade with losartan increased human abdominal subcutaneous ATBF [69]. In line, long-term RAS blockade with valsartan increased both fasting and postprandial ATBF [61] and may have contributed to the previously observed valsartan-induced increase in insulin sensitivity [70].

Taken together, the RAS may affect adipose tissue function by altering adipocyte size and lipid metabolism, inflammation and adipose tissue blood flow, thereby contributing to the development of both insulin resistance and type 2 diabetes. However, the metabolic effects of the RAS go beyond the adipose tissue and also affect skeletal muscle and the pancreas. 


\section{The renin-angiotensin system and skeletal muscle metabolism}

An increased RAS activity may cause impairments in skeletal muscle metabolism by affecting tissue perfusion, insulin signaling and mitochondrial function. The vascular system delivers nutrients and hormones to the skeletal muscle and local tissue perfusion may regulate skeletal muscle metabolism and contractile performance. Local infusion of ANG II in the gastrocnemius muscle reduced skeletal muscle blood flow in both lean and obese individuals [62], while chronic RAS blockade, with the ACE inhibitor (ACEi) captopril, increased postprandial forearm blood flow in subjects with type 2 diabetes [71]. However, an increased basal and insulin-stimulated total forearm blood flow was not observed after 2 weeks of ACEi treatment in obese insulin resistant subjects [72]. Thus, it is not completely clear whether the RAS plays a major role in the regulation of total skeletal muscle blood flow and/or microvascular function under physiological conditions.

As reviewed elsewhere [50,52], evidence suggest that the RAS may contribute to impaired insulin signaling in skeletal muscle, either directly or indirectly via the induction of oxidative stress. Importantly, most results are obtained from rodent studies and need further investigation in humans.

An impaired mitochondrial function in skeletal muscle has been suggested to contribute to the development of insulin resistance and type 2 diabetes, but whether reduced mitochondrial function is a cause or consequence of these disturbances remains to be established [73]. Nevertheless, chronic ANG II infusion in mice increased mitochondrial reactive oxygen species, decreased expression of genes involved in mitochondrial biogenesis and reduced mitochondrial content in C2C12 myocytes [74]. Furthermore, ANG II reduced muscle mitochondrial content, increased intramuscular triacylglycerol concentrations and reduced glycemic control in mice, while RAS blockade partially reversed these effects, leading to an increased fat oxidation, a decreased intramuscular triacylglycerol concentration and an improved glucose tolerance [74]. In contrast, treatment with the ACEi ramipril for two weeks had no significant effects on whole-body substrate oxidation, intramuscular triacylglycerol content and insulin sensitivity in obese insulin resistant men [72].

\section{The renin-angiotensin system and pancreatic beta-cell function}

Evidence suggests that elevated RAS activity may also contribute to an impaired insulin secretion [50]. In rodents, RAS activation reduced pancreatic islet blood flow, induced pancreatic islet fibrosis, oxidative stress, inflammation and impaired insulin secretion, whereas RAS blockade, with ACEi or ARB, improved pancreatic islet functionality and morphology and increased glucose tolerance, as reviewed [50]. Furthermore, in patients with impaired glucose metabolism, long-term RAS blockade, with valsartan, showed beneficial effects on pancreatic insulin secretion [70].

Taken together, the RAS may affect adipose tissue, skeletal muscle and pancreatic function and metabolism via different mechanisms, thereby contributing to the development of insulin resistance, an impaired insulin secretion and type 2 diabetes. Therefore, blockade of the RAS may exert protective effects against the development of metabolic disturbances. 


\section{Pharmacological modulation of the renin-angiotensin system}

Meta-analyses of comparative outcome trials have shown that RAS blockade, with either ACEi or ARB, reduced the incidence of new-onset type 2 diabetes by 20$30 \%$ in populations at high risk for developing type 2 diabetes [75, 76]. More recently, the prospective NAVIGATOR trial also showed that ARB treatment with valsartan, in addition to lifestyle modification, reduced the incidence of type 2 diabetes by $14 \%$ in subjects with impaired glucose homeostasis after a median follow-up of 5.3 years [77]. However, less pronounced improvements in glucose metabolism have been shown by the prospective DREAM trial [78]. In this trial, ACE inhibition, compared to placebo, non-significantly reduced the incidence of type 2 diabetes by $9 \%$ after a median follow-up of 3 years in subjects with impaired glucose homeostasis but without cardiovascular disease. Nevertheless, ACEi treatment significantly reduced 2-hour glucose concentrations and increased regression to normoglycemia [78]. Differences in study design, population and treatment duration may underlie the somewhat different outcomes in the DREAM trial and NAVIGATOR trials. Taken together, most randomized clinical trials indicate that RAS blockade may protect against the development of type 2 diabetes $[75,76]$.

\section{THE NATRIURETIC PEPTIDE SYSTEM}

Although data are not entirely consistent [79, 80], several community-based cohort studies have shown a lower activity of the natriuretic peptide (NP) system in obese subjects, which has been shown to be related the development of type 2 diabetes and chronic metabolic diseases [81-83]. As described below, modulation of the NP system can be protective against the development of insulin resistance and type 2 diabetes via multiple mechanisms, making it an interesting target to combat metabolic diseases.

The natriuretic peptide system contains several hormones of which Atrial NP (ANP), Brain-type NP (BNP) and C-type NP (CNP) are the most abundant ones. ANP and BNP are secreted from the cardiac atria [84] and ventricles [79], respectively, in response to cardiac wall stress, but also via other factors, such as weight loss [85], exercise [86], cold exposure [87] and hypoxia [88]. They bind to guanylyl cyclase-coupled receptors: type A (NPRA) and type B (NPRB), which are expressed in several tissues, including the adipose tissue [89]. While NPRA mediates the majority of the metabolic effects of ANP and BNP, type-C NP receptor (NPRC) is known as a membrane-bound clearance receptor that binds and incorporates circulating NP into cytoplasm where NP are inactivated [90, 91]. In addition to the NPRC-mediated degradation, NP are also degraded via extracellular proteases, such as the insulin-degrading enzyme [92] and neprilysin (NEP: neutral endopeptidase 24.11) [93] of which the latter is mainly expressed in the kidneys, but also present in adipocytes [94].

The lower NP effects in obesity and type 2 diabetes can be explained by a reduced cardiac NP secretion, a reduced tissue NPRA signaling and/or an increased systemic and tissue clearance, leading to reduced circulating NP concentrations. These reduced NP concentrations have been implicated in the development of insulin resistance and type 2 diabetes [83, 95-98], while higher NP concentrations 
are associated with a lower prevalence of new-onset type 2 diabetes [99, 100]. Beside changes in circulating NP concentrations, changes in the NP-receptors have also been observed. A decreased NPRA receptor expression at the level of abdominal subcutaneous adipose tissue, together with an increased NPRC receptor expression at both abdominal subcutaneous and omental adipose tissue has been observed in obese subjects with or without type 2 diabetes as compared to lean individuals, thereby altering the NPRA/NPRC ratio [80, 101-104]. This altered receptor expression, in combination with an increased NEP expression in adipose tissue of obese and insulin resistant subjects [94], may cause an increased NP clearance, leading to reduced NP bioavailability, thereby contributing to the development of cardiometabolic disturbances.

\section{Metabolic effects of the natriuretic peptide system}

The NP system was primarily known for the regulation of blood pressure and its physiological effects on the cardiovascular system, body fluid and electrolyte homeostasis [105, 106]. Nowadays, it is established that the NP system also exert effects on several key metabolic organs such as the adipose tissue and skeletal muscle, as reviewed elsewhere [107-109].

\section{The natriuretic peptide system and adipose tissue function}

The natriuretic peptides have been shown to stimulate lipolysis in human adipocytes, with ANP as the strongest effector, followed by BNP and CNP [110]. ANP and BNP stimulated in vitro lipolysis as much as the non-selective $\beta$ adrenergic receptor agonist isoproterenol, while in situ microdialysis experiments confirmed these NP-mediated lipolytic effects in abdominal subcutaneous adipose tissue of healthy young men [110]. Even under local $\alpha_{2^{-}}$and $\beta_{1 / 2}$-adrenergic blockade, a substantial non-adrenergic-mediated lipolysis was observed in subcutaneous adipose tissue of healthy young lean [111] and overweight men [112]. Intravenous infusion of ANP also acutely increased plasma concentrations of glycerol and free fatty acids (FFA) in young healthy lean and obese men, independently of the activation of the sympathetic nervous system [113]. Beside lipid mobilization, intravenous infusion of ANP also rapidly increased lipid oxidation in healthy normal weight men, both in the fasted [114, 115] and postprandial state [116]. When directly infused into the human subcutaneous adipose tissue of young lean men, ANP increased extracellular glycerol concentration and enhanced adipose tissue blood flow [110], together contributing to an increased lipid mobilization. This NP-mediated lipolysis revealed to be a cyclic guanosine monophosphate/protein kinase G (cGMP/PKG) dependent pathway, which induces the phosphorylation of perilipin 1 and hormone sensitive lipase [117].

ANP-treated human adipocytes also showed an increased activation of AMPprotein kinase (AMPK), a major metabolic energy sensor and master regulator of metabolic homeostasis [118]. Indeed, ANP increased energy expenditure and oxidative capacity and also increased mitochondrial biogenesis and function in differentiated human adipocytes [87, 118].

Beside effects on adipose tissue lipid mobilization and oxidation, NP have also been shown to possess anti-inflammatory properties $[119,120]$, to induce expression and secretion of the insulin-sensitizing factor adiponectin in both 
chronic heart failure patients [121] and healthy men [122], and to reduce systemic leptin concentrations in healthy men [123].

The natriuretic peptide system and skeletal muscle metabolism

In skeletal muscle, a NP-induced increase in peroxisome proliferator-activated receptor-gamma coactivator-1 alpha (PGC-1 $\alpha$ ) and proliferator-activated receptordelta (PPAR- $\delta$ ) mRNA expression as well as an increase in mitochondrial mass has been observed in $\mathrm{C} 2 \mathrm{C} 12$ myocytes [124]. In line, in human myotubes, ANP caused an increase in PGC-1a mRNA expression, which was paralleled by an upregulation of several genes and proteins involved in oxidative phosphorylation (OXPHOS), although mitochondrial proliferation and mitochondrial mass were not affected by ANP treatment [125].

Taken together, these findings suggest that NP may enhance the oxidative capacity of skeletal muscle.

The natriuretic peptide system and pancreatic beta-cell function

At the level of the pancreas, intravenous infusion of ANP increased insulin secretion in human subjects $[114,116,126]$ and improved pancreatic $\beta$-cell function in rodents [127]. ANP directly enhanced glucose-stimulated insulin secretion in cultured pancreatic islets of Langerhans [127] and induced $\beta$-cell growth in isolated rat pancreatic islets [128].

Interestingly, NP treatment may also induce changes at the level of the gastrointestinal tract. Intravenous BNP infusion, in young healthy lean men, showed a fasting-induced increase in total and acylated ghrelin concentrations, decreased the subjective rating of hunger and increased the feeling of satiety, without significantly changing circulating peptide YY, glucagon-like peptide 1, oxyntomodulin, pancreatic polypeptide, leptin and adiponectin concentrations [129]. Furthermore, intravenous infusion of BNP showed a decreased gastric emptying in a rodent model [130]. Also a novel gut-heart crosstalk has been described in rodents in which ANP mediates the blood pressure-lowering effects of glucagon-like peptide-1 (GLP-1) agonists [131]. The GLP-1 agonist, liraglutide, induced ANP secretion from the cardiac atrium and the blood pressure lowering effect of liraglutide was abrogated in Nppa (ANP) knockout mice.

Taken together, these data clearly show the involvement of the NP system in metabolic homeostasis and indicate that modulation of the NP system can be protective against the development of insulin resistance and type 2 diabetes via multiple mechanisms. Targeting the NP system may therefore serve as an effective strategy to combat these metabolic diseases. 


\section{PHARMACOLOGICAL MODULATION OF THE RENIN-ANGIOTENSIN SYSTEM}

\section{AND THE NATRIURETIC PEPTIDE SYSTEM}

As discussed above, obesity, type 2 diabetes and cardiovascular diseases are interrelated and may be characterized by a reduced NP system activity, an increased RAS activity as well as similar metabolic impairments. Recently, a novel dual-acting drug (sacubitril/valsartan) has been developed that combines ARB and NEP inhibition and facilitates beneficial effects of NP and other neprilysin substrates, while inhibiting the detrimental effects of the RAS [132]. In this respect, the latter cardiovascular medication, targeting the RAS and NP system, may also have positive metabolic effects. Moreover, the dual-acting mechanism of sacubitril/valsartan may have synergistic beneficial effects on several metabolic parameters. As described earlier in this thesis, the ARB valsartan improved insulin sensitivity [70] and beneficially affects adipocyte size and lipid metabolism, inflammation and adipose tissue blood flow [61]. The additional effects of the neprilysin inhibitor, sacubitril, may further enhance metabolic health (i.e. insulin sensitivity) via the increased NP concentrations, which also beneficially affect lipid mobilization from adipose tissue [114], postprandial lipid oxidation [116], adiponectin release and muscular oxidative capacity [125, 133]. Combined, sacubitril/valsartan facilitates beneficial effects of NP and other neprilysin substrates, while inhibiting the detrimental effects of the RAS. However, until recently, the metabolic effects of sacubitril/valsartan have not been investigated. In chapters 3,4 and 5 of this thesis, the effects of sacubitril/valsartan on metabolic parameters, such as peripheral insulin sensitivity, whole-body and local subcutaneous adipose tissue lipolysis as well as energy expenditure and substrate oxidation were investigated in obese hypertensive patients [134].

\section{LIFESTYLE INTERVENTION}

The management and treatment of obesity and obesity-related disorders is more complex than only achieving weight loss. The rational should not only focus on the maintenance of fat mass loss, but also on prolonged cardiometabolic risk reduction and health improvement. Lifestyle characteristics such as diet, physical inactivity, smoking, alcohol consumption and stress are important factors that influence the development of obesity and related comorbidities, and guidelines recommend changes in these lifestyle characteristics for both prevention and management of metabolic disease [135].

Manipulation of diet and physical activity levels are the first-choice interventions to reverse metabolic disturbances. Even though several large lifestyle intervention trials did not show significant beneficial effects of intensive exercise or combined diet and exercise lifestyle interventions on reducing the risk for cardiovascular outcomes [136, 137], other trials did observe beneficial effects on modifiable risk factors for cardiovascular disease [138], type 2 diabetes incidence and the metabolic syndrome [139]. This indicates that lifestyle interventions do have beneficial effects. Several important large lifestyle intervention trials that used dietary advice, exercise strategies and/or combinations have been performed to prevent type 2 diabetes. In this respect, the Malmö-study [140,141], the Chinese 
Da Qing IGT and Diabetes study [142], the Finnish Diabetes Prevention Study (DPS) [143], the Diabetes Prevention Program in the USA (DPP) [144] and the Study on Lifestyle intervention in Impaired glucose tolerance Maastricht (SLIM) [145] indicated that the incidence of type 2 diabetes was reduced by $40-60 \%$ following intensive (combined) diet and exercise intervention program. Importantly, long-term follow-up of these trials revealed that diabetes risk reduction still existed after 3 to 14 years after cessation of the intervention program [146-149]. Thus, lifestyle interventions clearly show improvements in a variety of health outcomes related to cardiometabolic health and can reduce the incidence of diseases [150]. However, the exact effects of the different components of the lifestyle program are not fully elucidated. While one study revealed that the combination of diet and exercise was more beneficial in reducing insulin resistance [151], another study showed that diet and exercise were equally effective compared to exercise alone to prevent the progression towards type 2 diabetes [142]. Moreover, another study found that the combination of diet and exercise was more effective than either treatment alone [145]. Nevertheless, combined dietary and exercise interventions have been shown to be most effective in reducing body weight [152].

Clearly, both prevention and management of disease progression can be achieved by strategies such as increased physical activity as well as dietary manipulation via a hypocaloric diet or an improved nutritional composition [153]. Interestingly, several dietary components, such as fatty acids, polyphenols and fibers may modulate fatty acid metabolism in tissues like skeletal muscle, liver and pancreas, both acute (i.e. postprandial phase) and more long-term, ultimately improving glucose metabolism, as extensively reviewed in chapter 2 [34]. Physical activity strategies include increased habitual physical activity (e.g. sitting less) and physical exercise levels (e.g. cycling) which lead to increased energy expenditure, improved physical fitness $[154,155]$ and contribute to an improved metabolic health. The exercise-induced improvements in metabolic risk profile have largely been attributed to changes in skeletal muscle metabolism and function, but physical exercise is likely to induce alterations in almost all metabolically active tissues, including the adipose tissue, as will be discussed below.

\section{Physical activity-induced effects on adipose tissue metabolism}

While physical activity is defined as any movement exerted by the skeletal muscle to increase resting energy expenditure, exercise is usually defined as a part of physical activity that is planned and/or structured [156]. When other aspects of energy expenditure or energy intake are not changed, increased physical activity and exercise induce a negative energy balance, resulting in a reduced fat mass [157]. This reduced fat mass depends on the accumulated effect of each bout of exercise and is caused when adipose tissue lipolysis and oxidation exceeds fat storage. Even though, physical activity and exercise-induced effects on adipose tissue metabolism are still not completely understood, several rodent studies suggest that exercise training may improve adipose tissue metabolism and function [158]. However, human studies that investigated the effects of exercise training on the adipose tissue function are scarce and need further investigation [157-159].

The exercise-induced fatty acid mobilization from adipose tissue is influenced by fatty acid re-esterification, adipose tissue lipolysis and adipose tissue blood flow 
(ATBF). A part of the exercise-induced increase of fatty acid mobilization can be attributed to a decreased rate of fatty acid re-esterification [160, 161] and an increased ATBF [162]. The exact mechanism for the increased ATBF is currently unknown and could be explained by an increased cardiac output or by other factors such as increases in circulating catecholamines and natriuretic peptides concentrations [163-165]. This increased ATBF contributes to an increased supply of hormones and signaling molecules (e.g. catecholamines, myokines) to the adipose tissue as well as the supply of mobilized FFA and adipokines to other tissues, such as skeletal muscle [166].

Adipose tissue lipolysis is mostly stimulated by low-intensity exercise and does not increase further at higher exercise intensities [167-169]. This process depends on both the adrenergic pathway, in which catecholamines (adrenalin and noradrenalin) bind to the adrenergic receptors, and the non-adrenergic pathways, that consist of the natriuretic peptide system, insulin, growth hormone and cortisol. In obesity, a blunted catecholamine-mediated lipolysis has been observed in subcutaneous adipose tissue $[170,171]$, in which the anti-lipolytic $\alpha_{2}$-adrenergic receptor becomes more predominant compared to the lipolytic $\beta_{1,2}$-adrenergic receptors [172, 173]. Also, as described earlier in this thesis, the natriuretic peptide concentrations are reduced in obesity and may contribute to an altered exerciseinduced lipolysis. Interestingly, after blocking the $\beta$-adrenergic receptor in subcutaneous adipose tissue during exercise in overweight men, it has been shown that adrenalin is not the primary lipolytic stimulus and that ANP might be of more importance in overweight men [112]. Noteworthy, several cross-sectional studies and exercise intervention studies have shown an increased lipolytic response in isolated adipocytes of active individuals compared to their sedentary counterparts [174-176] or as result of exercise training [177-179], reflecting an increased capacity for lipolysis at the cellular level. Furthermore, there is some evidence that exercise-induced adipose tissue lipolysis is increased with training [157], which is reflected by a similar or even greater lipolysis in spite of lower circulating concentrations of lipolytic hormones during exercise [180-182]. In line, exercise training has been shown to improve the $\beta$-adrenergic responsiveness in overweight [164] and obese men [183] and to improve the natriuretic peptide sensitivity in overweight men [164], which ultimately leads to an increased lipolysis. However, results are not always consistent and are complicated by confounding factors such as recent energy balance [157]. Nevertheless, in general, there seems to be an improved adipose tissue lipolytic sensitivity after training, which causes an increased fat mobilization and may reduce fat mass when the mobilized FFA are oxidized.

Several rodent studies have shown that exercise training may increase adipose tissue mitochondrial biogenesis [184, 185] and function [186-188] and induce browning of white adipose tissue [184, 185, 187, 189, 190], leading to an increased energy expenditure. In line, exercise training in human studies has been shown to increase white adipose tissue gene expression of PGC-1a [191] and oxidative metabolism markers [192], suggesting a higher mitochondrial capacity to oxidize fatty acids.

This increased mitochondrial oxidation can contribute to a reduced fat mass and reduced adipocyte size, which has beneficial effects on disease progression [193] and may lead to an altered adipokine expression [194]. Several rodent studies 
have shown that exercise training may beneficially alter adipokine expression [195, 196], but human data are conflicting. Although there is consistent evidence that exercise increases adipose tissue interleukin-6 (IL-6) expression and secretion after a single bout of exercise [197-199], results of other adipokines, such as adiponectin, leptin, TNF- $\alpha$ after a single bout of exercise or after exercise training are currently limited and conflicting [181, 200-206].

Collectively, these data may suggest that exercise improves adipose tissue metabolism and function and can ultimately contribute to a reduced disease progression and improved peripheral insulin sensitivity. Indeed, transplantation of white adipose tissue from trained animals to untrained recipients markedly improved skeletal muscle glucose uptake [187], suggesting that improvement of adipose tissue function may contribute to the increased peripheral insulin sensitivity after exercise training.

In chapters 6 and 7 of this thesis, the exercise training-induced effects on abdominal subcutaneous adipose tissue metabolism and lipolysis were investigated in obese and lean subjects.

\section{THESIS OUTLINE}

This thesis describes the effects of a pharmacological intervention as well as physical exercise interventions to improve metabolic health in obese individuals, with a focus on adipose tissue metabolism.

As described earlier in this thesis, adipose tissue dysfunction contributes to the development of insulin resistance and impaired glucose metabolism. By targeting fatty acid metabolism in the adipose tissue, liver, skeletal muscle or even the pancreas and the intestine, insulin sensitivity and glucose homeostasis may be improved. In Chapter 2, an extensive overview is provided of the fatty acid metabolism-related pathways in several metabolically active organs that can be targeted by dietary interventions, thereby improving whole-body glucose metabolism and insulin sensitivity.

Targeting the renin angiotensin system and the natriuretic peptide system by cardiovascular medication may improve adipose tissue and metabolic dysfunction, next to its effect on the cardiovascular system and hypertension. To investigate this, we conducted in Chapter 3, a multicenter, randomized, double-blind, doubledummy, parallel-group study to examine the metabolic effects of sacubitril/valsartan, which is a first-in-class angiotensin receptor neprilysin inhibitor, in obese hypertensive patients. By means of a hyperinsulinemic-euglycemic glucose clamp, we investigated the effects on peripheral insulin sensitivity, while whole-body lipolysis was determined using a stable isotope tracer $\left(\left[1,1,2,3,3-{ }^{2} \mathrm{H}\right]-\right.$ glycerol) and abdominal subcutaneous adipose tissue lipolysis was measured with the microdialysis technique. In addition to the collection of adipose tissue biopsies, we also performed indirect calorimetry measurements to assess energy expenditure and substrate utilization in these patients.

Chapter 4 extends the outcomes from the previous chapter and describes the effects of the 8-weeks treatment with sacubitril/valsartan compared to amlodipine on whole-body and adipose tissue lipolysis and lipid oxidation during a single bout of exercise. 
To obtain more detailed insight into possible mechanisms underlying the findings described in chapters 3 and 4, we assessed adipose tissue gene expression patterns using microarray analysis and protein expression profiles of enzymes involved in lipolysis, the natriuretic peptide signaling pathway and mitochondrial oxidative phosphorylation complexes. The results from these analyses are provided in Chapter 5.

There is some evidence that exercise training may improve adipose tissue function, which may contribute to the reduced risk for developing obesity-related insulin resistance and other comorbidities. However, human studies that investigated the effects of exercise training on the adipose tissue function are limited. Chapter 6 addresses the results of a supervised, progressive, combined endurance and resistance exercise training intervention for 12 weeks in well-phenotyped, obese subjects. In this study, we investigated exercise training-induced effects on adipose tissue by measuring abdominal subcutaneous adipocyte morphology, gene and protein expression of markers related to adipose tissue function. Moreover, we determined the exercise training-induced effects on ex vivo adipocyte lipolysis.

The aim of the study, described in Chapter 7, was to elucidate the physiological role of ANP-mediated lipolysis in abdominal subcutaneous adipose tissue of middle-aged obese insulin sensitive, obese insulin resistant and age-matched lean insulin sensitive men. By means of local combined blockade of the $\alpha$ - and $\beta$ adrenergic receptors (using a microdialysis approach), abdominal subcutaneous adipose tissue lipolysis was investigated during a single bout of low-intensity endurance exercise. In addition, we examined whether a combined endurance and resistance exercise training intervention for 12 weeks could improve abdominal subcutaneous adipose tissue lipolysis in obese insulin resistant individuals.

The main conclusions from the studies described in this thesis are discussed in Chapter $\mathbf{8}$ and placed into a broader perspective, accompanied by suggestions for further research. 


\section{REFERENCES}

1. World Health Organization. Fact sheet: Obesity and overweight. Updated June 2016. 2016.

2. World Health Organization. World Health Statistics 2015, Geneva,2015 [Available from: www.who.int/gho/publications/world_health_statistics/2015/en/.

3. Di Cesare M, Bentham J, Stevens GA, Zhou B, Danaei G, Lu Y, Bixby H, Cowan MJ, Riley LM, Hajifathalian K, Fortunato L, Taddei C, Bennett JE, Ikeda N, Zhu D, Zimmermann E, J. ZC. Trends in adult body-mass index in 200 countries from 1975 to 2014: a pooled analysis of 1698 population-based measurement studies with 19.2 million participants. Lancet. 2016;387(10026):1377-96.

4. Despres JP, Lemieux I. Abdominal obesity and metabolic syndrome. Nature. 2006;444(7121):881-7.

5. Kahn SE, Hull RL, Utzschneider KM. Mechanisms linking obesity to insulin resistance and type 2 diabetes. Nature. 2006;444(7121):840-6.

6. Reaven GM. Banting lecture 1988. Role of insulin resistance in human disease. Diabetes. 1988;37(12):1595-607.

7. Porte D, Jr. Banting lecture 1990. Beta-cells in type II diabetes mellitus. Diabetes. 1991;40(2):166-80.

8. Van Gaal LF, Mertens IL, De Block CE. Mechanisms linking obesity with cardiovascular disease. Nature. 2006;444(7121):875-80.

9. Li L, Liu DW, Yan HY, Wang ZY, Zhao SH, Wang B. Obesity is an independent risk factor for non-alcoholic fatty liver disease: evidence from a meta-analysis of 21 cohort studies. Obes Rev. 2016;17(6):510-9.

10. Preiss K, Brennan L, Clarke D. A systematic review of variables associated with the relationship between obesity and depression. Obes Rev. 2013;14(11):906-18.

11. Guh DP, Zhang W, Bansback N, Amarsi Z, Birmingham CL, Anis AH. The incidence of co-morbidities related to obesity and overweight: a systematic review and metaanalysis. BMC Public Health. 2009;9:88.

12. Bhaskaran K, Douglas I, Forbes H, dos-Santos-Silva I, Leon DA, Smeeth L. Bodymass index and risk of 22 specific cancers: a population-based cohort study of 5.24 million UK adults. Lancet. 2014;384(9945):755-65.

13. Lauby-Secretan B, Scoccianti C, Loomis D, Grosse Y, Bianchini F, Straif K, International Agency for Research on Cancer Handbook Working G. Body Fatness and Cancer--Viewpoint of the IARC Working Group. N Engl J Med. 2016;375(8):7948.

14. Hruschka DJ, Hadley C. How much do universal anthropometric standards bias the global monitoring of obesity and undernutrition? Obes Rev. 2016;17(11):1030-39.

15. Tomiyama AJ, Hunger JM, Nguyen-Cuu J, Wells C. Misclassification of cardiometabolic health when using body mass index categories in NHANES 20052012. Int J Obes (Lond). 2016;40(5):883-6.

16. Karpe F, Pinnick KE. Biology of upper-body and lower-body adipose tissue--link to whole-body phenotypes. Nat Rev Endocrinol. 2015;11(2):90-100.

17. Rush EC, Goedecke JH, Jennings C, Micklesfield L, Dugas L, Lambert EV, Plank LD. BMI, fat and muscle differences in urban women of five ethnicities from two countries. Int J Obes (Lond). 2007;31(8):1232-9.

18. Unnikrishnan R, Anjana RM, Mohan V. Diabetes in South Asians: is the phenotype different? Diabetes. 2014;63(1):53-5.

19. Blundell JE, Dulloo AG, Salvador J, Fruhbeck G, BMI ESWGo. Beyond BMI-phenotyping the obesities. Obes Facts. 2014;7(5):322-8.

20. Nicholson JK, Holmes E, Kinross JM, Darzi AW, Takats Z, Lindon JC. Metabolic phenotyping in clinical and surgical environments. Nature. 2012;491(7424):384-92. 
21. Stefan N, Fritsche A, Schick F, Haring HU. Phenotypes of prediabetes and stratification of cardiometabolic risk. Lancet Diabetes Endocrinol. 2016;4(9):789-98.

22. Yumuk V, Tsigos C, Fried M, Schindler K, Busetto L, Micic D, Toplak H, Obesity Management Task Force of the European Association for the Study of O. European Guidelines for Obesity Management in Adults. Obes Facts. 2015;8(6):402-24.

23. Klein S, Fontana L, Young VL, Coggan AR, Kilo C, Patterson BW, Mohammed BS. Absence of an effect of liposuction on insulin action and risk factors for coronary heart disease. N Engl J Med. 2004;350(25):2549-57.

24. Fonseca V. Effect of thiazolidinediones on body weight in patients with diabetes mellitus. Am J Med. 2003;115 Suppl 8A:42S-8S.

25. Ganda OP. Lipoatrophy, lipodystrophy, and insulin resistance. Ann Intern Med. 2000;133(4):304-6.

26. Goossens $\mathrm{GH}$. The role of adipose tissue dysfunction in the pathogenesis of obesityrelated insulin resistance. Physiol Behav. 2008;94(2):206-18.

27. Rosen ED, Spiegelman BM. What we talk about when we talk about fat. Cell. 2014;156(1-2):20-44.

28. Snijder MB, Zimmet PZ, Visser M, Dekker JM, Seidell JC, Shaw JE. Independent and opposite associations of waist and hip circumferences with diabetes, hypertension and dyslipidemia: the AusDiab Study. Int $J$ Obes Relat Metab Disord. 2004;28(3):402-9.

29. Yusuf S, Hawken S, Ounpuu S, Bautista L, Franzosi MG, Commerford P, Lang CC, Rumboldt Z, Onen CL, Lisheng L, Tanomsup S, Wangai P, Jr., Razak F, Sharma AM, Anand SS, Investigators IS. Obesity and the risk of myocardial infarction in 27,000 participants from 52 countries: a case-control study. Lancet. 2005;366(9497):1640-9.

30. Unger RH, Clark GO, Scherer PE, Orci L. Lipid homeostasis, lipotoxicity and the metabolic syndrome. Biochim Biophys Acta. 2010;1801(3):209-14.

31. Virtue S, Vidal-Puig A. Adipose tissue expandability, lipotoxicity and the Metabolic Syndrome--an allostatic perspective. Biochim Biophys Acta. 2010;1801(3):338-49.

32. Snel M, Jonker JT, Schoones J, Lamb H, de Roos A, Pijl H, Smit JW, Meinders AE, Jazet IM. Ectopic fat and insulin resistance: pathophysiology and effect of diet and lifestyle interventions. Int J Endocrinol. 2012;2012:983814.

33. Jocken JW, Goossens GH, Blaak EE. Targeting adipose tissue lipid metabolism to improve glucose metabolism in cardiometabolic disease. EMJ Diabet. 2014;2:73-82.

34. Stinkens R, Goossens GH, Jocken JW, Blaak EE. Targeting fatty acid metabolism to improve glucose metabolism. Obes Rev. 2015;16(9):715-57.

35. Bluher M. The distinction of metabolically 'healthy' from 'unhealthy' obese individuals. Curr Opin Lipidol. 2010;21(1):38-43.

36. van Vliet-Ostaptchouk JV, Nuotio ML, Slagter SN, Doiron D, Fischer K, Foco L, Gaye A, Gogele M, Heier M, Hiekkalinna T, Joensuu A, Newby C, Pang C, Partinen E, Reischl E, Schwienbacher C, Tammesoo ML, Swertz MA, Burton P, Ferretti V, Fortier I, Giepmans L, Harris JR, Hillege HL, Holmen J, Jula A, Kootstra-Ros JE, Kvaloy K, Holmen TL, Mannisto S, Metspalu A, Midthjell K, Murtagh MJ, Peters A, Pramstaller PP, Saaristo T, Salomaa V, Stolk RP, Uusitupa M, van der Harst P, van der Klauw $\mathrm{MM}$, Waldenberger M, Perola M, Wolffenbuttel $\mathrm{BH}$. The prevalence of metabolic syndrome and metabolically healthy obesity in Europe: a collaborative analysis of ten large cohort studies. BMC Endocr Disord. 2014;14:9.

37. Stefan N, Haring $\mathrm{HU}$, Hu FB, Schulze MB. Metabolically healthy obesity: epidemiology, mechanisms, and clinical implications. Lancet Diabetes Endocrinol. 2013;1(2):152-62.

38. Bluher $\mathrm{M}$. Are metabolically healthy obese individuals really healthy? Eur J Endocrinol. 2014;171(6):R209-19.

39. Lavie CJ, De Schutter A, Milani RV. Healthy obese versus unhealthy lean: the obesity paradox. Nat Rev Endocrinol. 2015;11(1):55-62. 
40. Rey-Lopez JP, de Rezende LF, Pastor-Valero M, Tess BH. The prevalence of metabolically healthy obesity: a systematic review and critical evaluation of the definitions used. Obes Rev. 2014;15(10):781-90.

41. Bell JA, Kivimaki M, Hamer M. Metabolically healthy obesity and risk of incident type 2 diabetes: a meta-analysis of prospective cohort studies. Obes Rev. 2014;15(6):504-15.

42. Kramer CK, Zinman B, Retnakaran R. Are metabolically healthy overweight and obesity benign conditions?: A systematic review and meta-analysis. Ann Intern Med. 2013;159(11):758-69.

43. Eckel N, Meidtner K, Kalle-Uhlmann T, Stefan N, Schulze MB. Metabolically healthy obesity and cardiovascular events: A systematic review and meta-analysis. Eur $\mathrm{J}$ Prev Cardiol. 2016;23(9):956-66.

44. Appleton SL, Seaborn CJ, Visvanathan R, Hill CL, Gill TK, Taylor AW, Adams RJ, North West Adelaide Health Study T. Diabetes and cardiovascular disease outcomes in the metabolically healthy obese phenotype: a cohort study. Diabetes Care. 2013;36(8):2388-94.

45. Ryden M, Hrydziuszko O, Mileti E, Raman A, Bornholdt J, Boyd M, Toft E, Qvist V, Naslund E, Thorell A, Andersson DP, Dahlman I, Gao H, Sandelin A, Daub CO, Arner P. The Adipose Transcriptional Response to Insulin Is Determined by Obesity, Not Insulin Sensitivity. Cell Rep. 2016;16(9):2317-26.

46. Munoz-Garach A, Cornejo-Pareja I, Tinahones FJ. Does Metabolically Healthy Obesity Exist? Nutrients. 2016;8(6).

47. Goossens GH. The Metabolic Phenotype in Obesity: Fat Mass, Body Fat Distribution, and Adipose Tissue Function. Obes Facts. 2017;10(3):207-15.

48. Bell JA, Shipley MJ, Kivimaki M. Healthy obesity is not safe obesity. Int J Obes (Lond). 2016;40(8):1333.

49. Berard E, Niel O, Rubio A. Is the renin-angiotensin system actually hypertensive? Pediatr Nephrol. 2014;29(6):951-60.

50. Goossens GH. The renin-angiotensin system in the pathophysiology of type 2 diabetes. Obes Facts. 2012;5(4):611-24.

51. Frigolet ME, Torres N, Tovar AR. The renin-angiotensin system in adipose tissue and its metabolic consequences during obesity. J Nutr Biochem. 2013;24(12):2003-15.

52. Ramalingam L, Menikdiwela K, LeMieux M, Dufour JM, Kaur G, Kalupahana N, Moustaid-Moussa N. The renin angiotensin system, oxidative stress and mitochondrial function in obesity and insulin resistance. Biochim Biophys Acta. 2016.

53. Borghi F, Seva-Pessoa B, Grassi-Kassisse DM. The adipose tissue and the involvement of the renin-angiotensin-aldosterone system in cardiometabolic syndrome. Cell Tissue Res. 2016;366(3):543-8.

54. Goossens GH, Blaak EE, van Baak MA. Possible involvement of the adipose tissue renin-angiotensin system in the pathophysiology of obesity and obesity-related disorders. Obes Rev. 2003;4(1):43-55.

55. Unger $T$. The role of the renin-angiotensin system in the development of cardiovascular disease. Am J Cardiol. 2002;89(2A):3A-9A; discussion 10A.

56. Danser AH. Local renin-angiotensin systems. Mol Cell Biochem. 1996;157(1-2):2116.

57. Darimont C, Vassaux G, Gaillard D, Ailhaud G, Negrel R. In situ microdialysis of prostaglandins in adipose tissue: stimulation of prostacyclin release by angiotensin II. Int J Obes Relat Metab Disord. 1994;18(12):783-8.

58. Jones BH, Standridge MK, Moustaid N. Angiotensin II increases lipogenesis in 3T3L1 and human adipose cells. Endocrinology. 1997;138(4):1512-9.

59. Janke J, Engeli S, Gorzelniak K, Luft FC, Sharma AM. Mature adipocytes inhibit in vitro differentiation of human preadipocytes via angiotensin type 1 receptors. Diabetes. 2002;51(6):1699-707. 
60. Schling P, Loffler G. Effects of angiotensin II on adipose conversion and expression of genes of the renin-angiotensin system in human preadipocytes. Horm Metab Res. $2001 ; 33(4): 189-95$.

61. Goossens GH, Moors CC, van der Zijl NJ, Venteclef N, Alili R, Jocken JW, Essers Y, Cleutjens JP, Clement K, Diamant M, Blaak EE. Valsartan improves adipose tissue function in humans with impaired glucose metabolism: a randomized placebocontrolled double-blind trial. PLoS One. 2012;7(6):e39930.

62. Goossens GH, Blaak EE, Saris WH, van Baak MA. Angiotensin II-induced effects on adipose and skeletal muscle tissue blood flow and lipolysis in normal-weight and obese subjects. J Clin Endocrinol Metab. 2004;89(6):2690-6.

63. Boschmann M, Ringel J, Klaus S, Sharma AM. Metabolic and hemodynamic response of adipose tissue to angiotensin II. Obes Res. 2001;9(8):486-91.

64. Boschmann M, Rosenbaum M, Leibel RL, Segal KR. Metabolic and hemodynamic responses to exercise in subcutaneous adipose tissue and skeletal muscle. Int $\mathrm{J}$ Sports Med. 2002;23(8):537-43.

65. Goossens GH, Blaak EE, Arner P, Saris WH, van Baak MA. Angiotensin II: a hormone that affects lipid metabolism in adipose tissue. Int $\mathrm{J}$ Obes (Lond). 2007;31(2):382-4.

66. Boschmann M, Adams F, Schaller K, Franke G, Sharma AM, Klaus S, Luft FC, Jordan J. Hemodynamic and metabolic responses to interstitial angiotensin II in normal weight and obese men. J Hypertens. 2006;24(6):1165-71.

67. Boschmann M, Kreuzberg U, Engeli S, Adams F, Franke G, Klaua S, Scholze J, Weidinger G, Luft FC, Sharma AM, Jordan J. The effect of oral glucose loads on tissue metabolism during angiotensin II receptor and beta-receptor blockade in obese hypertensive subjects. Horm Metab Res. 2006;38(5):323-9.

68. Frayn KN, Karpe F. Regulation of human subcutaneous adipose tissue blood flow. Int J Obes (Lond). 2014;38(8):1019-26.

69. Goossens GH, McQuaid SE, Dennis AL, van Baak MA, Blaak EE, Frayn KN, Saris $\mathrm{WH}$, Karpe F. Angiotensin II: a major regulator of subcutaneous adipose tissue blood flow in humans. J Physiol. 2006;571(Pt 2):451-60.

70. van der Zijl NJ, Moors CC, Goossens GH, Hermans MM, Blaak EE, Diamant M. Valsartan improves \{beta\}-cell function and insulin sensitivity in subjects with impaired glucose metabolism: a randomized controlled trial. Diabetes Care. 2011;34(4):845-51.

71. Kodama J, Katayama S, Tanaka K, Itabashi A, Kawazu S, Ishii J. Effect of captopril on glucose concentration. Possible role of augmented postprandial forearm blood flow. Diabetes Care. 1990;13(11):1109-11.

72. Goossens GH, Blaak EE, Schiffers PM, Saris WH, van Baak MA. Effect of short-term ACE inhibitor treatment on peripheral insulin sensitivity in obese insulin-resistant subjects. Diabetologia. 2006;49(12):3009-16.

73. Hoeks J, Schrauwen P. Muscle mitochondria and insulin resistance: a human perspective. Trends Endocrinol Metab. 2012;23(9):444-50.

74. Mitsuishi M, Miyashita K, Muraki A, Itoh H. Angiotensin II reduces mitochondrial content in skeletal muscle and affects glycemic control. Diabetes. 2009;58(3):710-7.

75. Jandeleit-Dahm KA, Tikellis C, Reid CM, Johnston CI, Cooper ME. Why blockade of the renin-angiotensin system reduces the incidence of new-onset diabetes. $J$ Hypertens. 2005;23(3):463-73.

76. Gillespie EL, White CM, Kardas M, Lindberg M, Coleman Cl. The impact of ACE inhibitors or angiotensin II type 1 receptor blockers on the development of new-onset type 2 diabetes. Diabetes Care. 2005;28(9):2261-6.

77. McMurray JJ, Holman RR, Haffner SM, Bethel MA, Holzhauer B, Hua TA, Belenkov Y, Boolell M, Buse JB, Buckley BM, Chacra AR, Chiang FT, Charbonnel B, Chow CC, Davies MJ, Deedwania P, Diem P, Einhorn D, Fonseca V, Fulcher GR, Gaciong 
Z, Gaztambide S, Giles T, Horton E, Ilkova H, Jenssen T, Kahn SE, Krum H, Laakso M, Leiter LA, Levitt NS, Mareev V, Martinez F, Masson C, Mazzone T, Meaney E, Nesto R, Pan C, Prager R, Raptis SA, Rutten GE, Sandstroem H, Schaper F, Scheen A, Schmitz O, Sinay I, Soska V, Stender S, Tamas G, Tognoni G, Tuomilehto J, Villamil AS, Vozar J, Califf RM. Effect of valsartan on the incidence of diabetes and cardiovascular events. N Engl J Med. 2010;362(16):1477-90.

78. Bosch J, Yusuf S, Gerstein HC, Pogue J, Sheridan P, Dagenais G, Diaz R, Avezum A, Lanas F, Probstfield J, Fodor G, Holman RR. Effect of ramipril on the incidence of diabetes. N Engl J Med. 2006;355(15):1551-62.

79. Nannipieri M, Seghieri G, Catalano C, Prontera T, Baldi S, Ferrannini E. Defective regulation and action of atrial natriuretic peptide in type 2 diabetes. Horm Metab Res. 2002;34(5):265-70.

80. Dessi-Fulgheri P, Sarzani R, Tamburrini P, Moraca A, Espinosa E, Cola G, Giantomassi L, Rappelli A. Plasma atrial natriuretic peptide and natriuretic peptide receptor gene expression in adipose tissue of normotensive and hypertensive obese patients. J Hypertens. 1997;15(12 Pt 2):1695-9.

81. Wang TJ, Larson MG, Levy D, Benjamin EJ, Leip EP, Wilson PW, Vasan RS. Impact of obesity on plasma natriuretic peptide levels. Circulation. 2004;109(5):594-600.

82. Das SR, Drazner MH, Dries DL, Vega GL, Stanek HG, Abdullah SM, Canham RM, Chung AK, Leonard D, Wians FH, Jr., de Lemos JA. Impact of body mass and body composition on circulating levels of natriuretic peptides: results from the Dallas Heart Study. Circulation. 2005;112(14):2163-8.

83. Khan AM, Cheng S, Magnusson M, Larson MG, Newton-Cheh C, McCabe EL, Coviello AD, Florez JC, Fox CS, Levy D, Robins SJ, Arora P, Bhasin S, Lam CS, Vasan RS, Melander O, Wang TJ. Cardiac natriuretic peptides, obesity, and insulin resistance: evidence from two community-based studies. J Clin Endocrinol Metab. 2011;96(10):3242-9.

84. Nakao K, Ogawa Y, Suga S, Imura H. Molecular biology and biochemistry of the natriuretic peptide system. I: Natriuretic peptides. J Hypertens. 1992;10(9):907-12.

85. Chen-Tournoux A, Khan AM, Baggish AL, Castro VM, Semigran MJ, McCabe EL, Moukarbel G, Reingold J, Durrani S, Lewis GD, Newton-Cheh C, Scherrer-Crosbie M, Kaplan LM, Wang TJ. Effect of weight loss after weight loss surgery on plasma Nterminal pro-B-type natriuretic peptide levels. Am J Cardiol. 2010;106(10):1450-5.

86. Neumayr G, Pfister R, Mitterbauer G, Eibl G, Hoertnagl H. Effect of competitive marathon cycling on plasma $\mathrm{N}$-terminal pro-brain natriuretic peptide and cardiac troponin T in healthy recreational cyclists. Am J Cardiol. 2005;96(5):732-5.

87. Bordicchia M, Liu D, Amri EZ, Ailhaud G, Dessi-Fulgheri P, Zhang C, Takahashi N, Sarzani R, Collins S. Cardiac natriuretic peptides act via p38 MAPK to induce the brown fat thermogenic program in mouse and human adipocytes. J Clin Invest. 2012;122(3):1022-36.

88. Kawashima A, Kubo K, Hirai K, Yoshikawa S, Matsuzawa Y, Kobayashi T. Plasma levels of atrial natriuretic peptide under acute hypoxia in normal subjects. Respir Physiol. 1989;76(1):79-91.

89. Potter LR, Abbey-Hosch S, Dickey DM. Natriuretic peptides, their receptors, and cyclic guanosine monophosphate-dependent signaling functions. Endocr Rev. 2006;27(1):47-72.

90. Koh GY, Nussenzveig DR, Okolicany J, Price DA, Maack T. Dynamics of atrial natriuretic factor-guanylate cyclase receptors and receptor-ligand complexes in cultured glomerular mesangial and renomedullary interstitial cells. J Biol Chem. 1992;267(17):11987-94.

91. Nussenzveig DR, Lewicki JA, Maack T. Cellular mechanisms of the clearance function of type $C$ receptors of atrial natriuretic factor. $J$ Biol Chem. 1990;265(34):20952-8. 
92. Ralat LA, Guo Q, Ren M, Funke T, Dickey DM, Potter LR, Tang WJ. Insulindegrading enzyme modulates the natriuretic peptide-mediated signaling response. J Biol Chem. 2011;286(6):4670-9.

93. Potter LR. Natriuretic peptide metabolism, clearance and degradation. FEBS J. $2011 ; 278(11): 1808-17$.

94. Standeven KF, Hess K, Carter AM, Rice GI, Cordell PA, Balmforth AJ, Lu B, Scott DJ, Turner AJ, Hooper NM, Grant PJ. Neprilysin, obesity and the metabolic syndrome. Int J Obes (Lond). 2011;35(8):1031-40.

95. Walford GA, Ma Y, Christophi CA, Goldberg RB, Jarolim P, Horton E, Mather KJ, Barrett-Connor E, Davis J, Florez JC, Wang TJ, Diabetes Prevention Program Research G. Circulating natriuretic peptide concentrations reflect changes in insulin sensitivity over time in the Diabetes Prevention Program. Diabetologia. 2014;57(5):935-9.

96. Jujic A, Nilsson PM, Persson M, Holst JJ, Torekov SS, Lyssenko V, Groop L, Melander O, Magnusson M. Atrial Natriuretic Peptide in the High Normal Range Is Associated With Lower Prevalence of Insulin Resistance. J Clin Endocrinol Metab. 2016;101(4):1372-80.

97. Magnusson M, Jujic A, Hedblad B, Engstrom G, Persson M, Struck J, Morgenthaler NG, Nilsson P, Newton-Cheh C, Wang TJ, Melander O. Low plasma level of atrial natriuretic peptide predicts development of diabetes: the prospective Malmo Diet and Cancer study. J Clin Endocrinol Metab. 2012;97(2):638-45.

98. Lazo M, Young JH, Brancati FL, Coresh J, Whelton S, Ndumele CE, Hoogeveen R, Ballantyne CM, Selvin E. NH2-terminal pro-brain natriuretic peptide and risk of diabetes. Diabetes. 2013;62(9):3189-93.

99. Pfister R, Sharp S, Luben R, Welsh P, Barroso I, Salomaa V, Meirhaeghe A, Khaw KT, Sattar N, Langenberg C, Wareham NJ. Mendelian randomization study of B-type natriuretic peptide and type 2 diabetes: evidence of causal association from population studies. PLoS Med. 2011;8(10):e1001112.

100. Jujic A, Nilsson PM, Engstrom G, Hedblad B, Melander O, Magnusson M. Atrial natriuretic peptide and type 2 diabetes development--biomarker and genotype association study. PLoS One. 2014;9(2):e89201.

101. Pivovarova O, Gogebakan O, Kloting N, Sparwasser A, Weickert MO, Haddad I, Nikiforova VJ, Bergmann A, Kruse M, Seltmann AC, Bluher M, Pfeiffer AF, Rudovich $N$. Insulin up-regulates natriuretic peptide clearance receptor expression in the subcutaneous fat depot in obese subjects: a missing link between CVD risk and obesity? J Clin Endocrinol Metab. 2012;97(5):E731-9.

102. Ryden M, Backdahl J, Petrus P, Thorell A, Gao H, Coue M, Langin D, Moro C, Arner P. Impaired atrial natriuretic peptide-mediated lipolysis in obesity. Int $\mathrm{J}$ Obes (Lond). 2016;40(4):714-20.

103. Kovacova Z, Tharp WG, Liu D, Wei W, Xie H, Collins S, Pratley RE. Adipose tissue natriuretic peptide receptor expression is related to insulin sensitivity in obesity and diabetes. Obesity (Silver Spring). 2016;24(4):820-8.

104. Verboven K, Hansen D, Moro C, Eijnde BO, Hoebers N, Knol J, Bouckaert W, Dams A, Blaak EE, Jocken JW. Attenuated atrial natriuretic peptide-mediated lipolysis in subcutaneous adipocytes of obese type 2 diabetic men. Clin Sci (Lond). 2016;130(13):1105-14.

105. Clerico A, Vittorini S. The Cardiac Natriuretic Hormone System. In: Clerico A, Emdin M, editors. Natriuretic peptides: The Hormones of the Heart: Springer-Verlag Italia; 2006. p. 21-64.

106. Kerkela R, Ulvila J, Magga J. Natriuretic Peptides in the Regulation of Cardiovascular Physiology and Metabolic Events. J Am Heart Assoc. 2015;4(10):e002423. 
107. Schlueter N, de Sterke A, Willmes DM, Spranger J, Jordan J, Birkenfeld AL. Metabolic actions of natriuretic peptides and therapeutic potential in the metabolic syndrome. Pharmacol Ther. 2014;144(1):12-27.

108. Coue M, Moro C. Natriuretic peptide control of energy balance and glucose homeostasis. Biochimie. 2016;124:84-91.

109. Moro C. Targeting cardiac natriuretic peptides in the therapy of diabetes and obesity. Expert Opin Ther Targets. 2016;20(12):1445-52.

110. Sengenes C, Berlan M, De Glisezinski I, Lafontan M, Galitzky J. Natriuretic peptides: a new lipolytic pathway in human adipocytes. FASEB J. 2000;14(10):1345-51.

111. Moro C, Polak J, Hejnova J, Klimcakova E, Crampes F, Stich V, Lafontan M, Berlan M. Atrial natriuretic peptide stimulates lipid mobilization during repeated bouts of endurance exercise. Am J Physiol Endocrinol Metab. 2006;290(5):E864-9.

112. Moro C, Pillard F, de Glisezinski I, Klimcakova E, Crampes F, Thalamas C, Harant I, Marques MA, Lafontan M, Berlan M. Exercise-induced lipid mobilization in subcutaneous adipose tissue is mainly related to natriuretic peptides in overweight men. Am J Physiol Endocrinol Metab. 2008;295(2):E505-13.

113. Galitzky J, Sengenes C, Thalamas C, Marques MA, Senard JM, Lafontan M, Berlan $M$. The lipid-mobilizing effect of atrial natriuretic peptide is unrelated to sympathetic nervous system activation or obesity in young men. J Lipid Res. 2001;42(4):536-44.

114. Birkenfeld AL, Boschmann M, Moro C, Adams F, Heusser K, Franke G, Berlan M, Luft FC, Lafontan M, Jordan J. Lipid mobilization with physiological atrial natriuretic peptide concentrations in humans. J Clin Endocrinol Metab. 2005;90(6):3622-8.

115. Birkenfeld AL, Boschmann M, Moro C, Adams F, Heusser K, Tank J, Diedrich A, Schroeder C, Franke G, Berlan M, Luft FC, Lafontan M, Jordan J. Beta-adrenergic and atrial natriuretic peptide interactions on human cardiovascular and metabolic regulation. J Clin Endocrinol Metab. 2006;91(12):5069-75.

116. Birkenfeld AL, Budziarek P, Boschmann M, Moro C, Adams F, Franke G, Berlan M, Marques MA, Sweep FC, Luft FC, Lafontan M, Jordan J. Atrial natriuretic peptide induces postprandial lipid oxidation in humans. Diabetes. 2008;57(12):3199-204.

117. Sengenes C, Bouloumie A, Hauner H, Berlan M, Busse R, Lafontan M, Galitzky J. Involvement of a cGMP-dependent pathway in the natriuretic peptide-mediated hormone-sensitive lipase phosphorylation in human adipocytes. J Biol Chem. 2003;278(49):48617-26.

118. Souza SC, Chau MD, Yang Q, Gauthier MS, Clairmont KB, Wu Z, Gromada J, Dole WP. Atrial natriuretic peptide regulates lipid mobilization and oxygen consumption in human adipocytes by activating AMPK. Biochem Biophys Res Commun. 2011;410(3):398-403.

119. Kiemer AK, Vollmar AM. The atrial natriuretic peptide regulates the production of inflammatory mediators in macrophages. Ann Rheum Dis. 2001;60 Suppl 3:iii68-70.

120. Moro C, Klimcakova E, Lolmede K, Berlan M, Lafontan M, Stich V, Bouloumie A, Galitzky J, Arner P, Langin D. Atrial natriuretic peptide inhibits the production of adipokines and cytokines linked to inflammation and insulin resistance in human subcutaneous adipose tissue. Diabetologia. 2007;50(5):1038-47.

121. Tsukamoto O, Fujita M, Kato M, Yamazaki S, Asano Y, Ogai A, Okazaki H, Asai M, Nagamachi Y, Maeda N, Shintani Y, Minamino T, Asakura M, Kishimoto I, Funahashi $\mathrm{T}$, Tomoike $\mathrm{H}$, Kitakaze M. Natriuretic peptides enhance the production of adiponectin in human adipocytes and in patients with chronic heart failure. J Am Coll Cardiol. 2009;53(22):2070-7.

122. Birkenfeld AL, Boschmann M, Engeli S, Moro C, Arafat AM, Luft FC, Jordan J. Atrial natriuretic peptide and adiponectin interactions in man. PLoS One. 2012;7(8):e43238. 
123. Jordan J, Fischer-Posovszky $P$, Reinke J, Daniels $M$, Wabitsch $M$, Engeli $S$, Birkenfeld AL. A novel heart-adipose tissue axis: Atrial natriuretic peptide and leptin interactions in man. Diabetologie und Stoffwechsel. 2016(11 - LB8).

124. Miyashita K, Itoh H, Tsujimoto H, Tamura N, Fukunaga Y, Sone M, Yamahara K, Taura D, Inuzuka M, Sonoyama T, Nakao K. Natriuretic peptides/cGMP/cGMPdependent protein kinase cascades promote muscle mitochondrial biogenesis and prevent obesity. Diabetes. 2009;58(12):2880-92.

125. Engeli S, Birkenfeld AL, Badin PM, Bourlier V, Louche K, Viguerie N, Thalamas C, Montastier E, Larrouy D, Harant I, de Glisezinski I, Lieske S, Reinke J, Beckmann B, Langin D, Jordan J, Moro C. Natriuretic peptides enhance the oxidative capacity of human skeletal muscle. J Clin Invest. 2012;122(12):4675-9.

126. Uehlinger DE, Weidmann P, Gnadinger MP, Hasler L, Bachmann C, Shaw S, Hellmuller B, Lang RE. Increase in circulating insulin induced by atrial natriuretic peptide in normal humans. J Cardiovasc Pharmacol. 1986;8(6):1122-9.

127. Ropero AB, Soriano S, Tuduri E, Marroqui L, Tellez N, Gassner B, Juan-Pico P, Montanya E, Quesada I, Kuhn M, Nadal A. The atrial natriuretic peptide and guanylyl cyclase-A system modulates pancreatic beta-cell function. Endocrinology. 2010;151(8):3665-74.

128. You H, Laychock SG. Atrial natriuretic peptide promotes pancreatic islet beta-cell growth and Akt/Foxo1a/cyclin D2 signaling. Endocrinology. 2009;150(12):5455-65.

129. Vila G, Grimm G, Resl M, Heinisch B, Einwallner E, Esterbauer H, Dieplinger B, Mueller T, Luger A, Clodi M. B-type natriuretic peptide modulates ghrelin, hunger, and satiety in healthy men. Diabetes. 2012;61(10):2592-6.

130. Addisu A, Gower WR, Jr., Landon CS, Dietz JR. B-type natriuretic peptide decreases gastric emptying and absorption. Exp Biol Med (Maywood). 2008;233(4):475-82.

131. Kim M, Platt MJ, Shibasaki T, Quaggin SE, Backx PH, Seino S, Simpson JA, Drucker DJ. GLP-1 receptor activation and Epac2 link atrial natriuretic peptide secretion to control of blood pressure. Nat Med. 2013;19(5):567-75.

132. Langenickel T.H. DWP. Angiotensin receptor-neprilysin inhibition with LCZ696: a novel approach for the treatment of heart failure. Drug Discovery Today: Therapeutic Strategies. 2012;9(4):e131-e9.

133. Coue M, Badin PM, Vila IK, Laurens C, Louche K, Marques MA, Bourlier V, Mouisel E, Tavernier G, Rustan AC, Galgani JE, Joanisse DR, Smith SR, Langin D, Moro C. Defective natriuretic peptide receptor signaling in skeletal muscle links obesity to type 2 diabetes. Diabetes. 2015.

134. Jordan J, Stinkens R, Jax T, Engeli S, Blaak EE, May M, Havekes B, Schindler C, Albrecht D, Pal P, Heise T, Goossens GH, Langenickel T. Metabolic benefits of LCZ696 in obese hypertensive patients: A randomized, double-blind, activecontrolled, parallel-group study. [Poster presentation June 7th, 2015, American Diabetes Association 75th Scientific conference, Boston, Massachusetts]. In press 2015.

135. American Diabetes Association. Standards of Medical Care in Diabetes. Diabetes Care. 2016;39(Suppl 1).

136. Wing RR, Bolin P, Brancati FL, Bray GA, Clark JM, Coday M, Crow RS, Curtis JM, Egan CM, Espeland MA, Evans M, Foreyt JP, Ghazarian S, Gregg EW, Harrison B, Hazuda HP, Hill JO, Horton ES, Hubbard VS, Jakicic JM, Jeffery RW, Johnson KC, Kahn SE, Kitabchi AE, Knowler WC, Lewis CE, Maschak-Carey BJ, Montez MG, Murillo A, Nathan DM, Patricio J, Peters A, Pi-Sunyer X, Pownall H, Reboussin D, Regensteiner JG, Rickman AD, Ryan DH, Safford M, Wadden TA, Wagenknecht LE, West DS, Williamson DF, Yanovski SZ. Cardiovascular effects of intensive lifestyle intervention in type 2 diabetes. N Engl J Med. 2013;369(2):145-54.

137. O'Connor CM, Whellan DJ, Lee KL, Keteyian SJ, Cooper LS, Ellis SJ, Leifer ES, Kraus WE, Kitzman DW, Blumenthal JA, Rendall DS, Miller NH, Fleg JL, Schulman 
KA, McKelvie RS, Zannad F, Pina IL, Investigators H-A. Efficacy and safety of exercise training in patients with chronic heart failure: HF-ACTION randomized controlled trial. JAMA. 2009;301(14):1439-50.

138. Gaede P, Vedel P, Larsen N, Jensen GV, Parving HH, Pedersen O. Multifactorial intervention and cardiovascular disease in patients with type 2 diabetes. $\mathrm{N}$ Engl $\mathrm{J}$ Med. 2003;348(5):383-93.

139. den Boer AT, Herraets IJ, Stegen J, Roumen C, Corpeleijn E, Schaper NC, Feskens E, Blaak EE. Prevention of the metabolic syndrome in IGT subjects in a lifestyle intervention: results from the SLIM study. Nutr Metab Cardiovasc Dis. 2013;23(11):1147-53.

140. Eriksson KF, Lindgarde F. Prevention of type 2 (non-insulin-dependent) diabetes mellitus by diet and physical exercise. The 6-year Malmo feasibility study. Diabetologia. 1991;34(12):891-8.

141. Eriksson KF, Lindgarde F. No excess 12-year mortality in men with impaired glucose tolerance who participated in the Malmo Preventive Trial with diet and exercise. Diabetologia. 1998;41(9):1010-6.

142. Pan XR, Li GW, Hu YH, Wang JX, Yang WY, An ZX, Hu ZX, Lin J, Xiao JZ, Cao HB, Liu PA, Jiang XG, Jiang YY, Wang JP, Zheng $H$, Zhang $H$, Bennett $P H$, Howard BV. Effects of diet and exercise in preventing NIDDM in people with impaired glucose tolerance. The Da Qing IGT and Diabetes Study. Diabetes Care. 1997;20(4):537-44.

143. Tuomilehto J, Lindstrom J, Eriksson JG, Valle TT, Hamalainen H, Ilanne-Parikka P, Keinanen-Kiukaanniemi S, Laakso M, Louheranta A, Rastas M, Salminen V, Uusitupa M, Finnish Diabetes Prevention Study G. Prevention of type 2 diabetes mellitus by changes in lifestyle among subjects with impaired glucose tolerance. $\mathrm{N}$ Engl J Med. 2001;344(18):1343-50.

144. Knowler WC, Barrett-Connor E, Fowler SE, Hamman RF, Lachin JM, Walker EA, Nathan DM, Diabetes Prevention Program Research G. Reduction in the incidence of type 2 diabetes with lifestyle intervention or metformin. $\mathrm{N}$ Engl $\mathrm{J}$ Med. 2002;346(6):393-403.

145. Mensink M, Blaak EE, Corpeleijn E, Saris WH, de Bruin TW, Feskens EJ. Lifestyle intervention according to general recommendations improves glucose tolerance. Obes Res. 2003;11(12):1588-96.

146. Lindstrom J, Ilanne-Parikka P, Peltonen M, Aunola S, Eriksson JG, Hemio K, Hamalainen $H$, Harkonen $P$, Keinanen-Kiukaanniemi $S$, Laakso $M$, Louheranta $A$, Mannelin M, Paturi M, Sundvall J, Valle TT, Uusitupa M, Tuomilehto J, Finnish Diabetes Prevention Study G. Sustained reduction in the incidence of type 2 diabetes by lifestyle intervention: follow-up of the Finnish Diabetes Prevention Study. Lancet. 2006;368(9548):1673-9.

147. Li G, Zhang P, Wang J, Gregg EW, Yang W, Gong Q, Li H, Li H, Jiang Y, An Y, Shuai Y, Zhang B, Zhang J, Thompson TJ, Gerzoff RB, Roglic G, Hu Y, Bennett PH. The long-term effect of lifestyle interventions to prevent diabetes in the China $\mathrm{Da}$ Qing Diabetes Prevention Study: a 20-year follow-up study. Lancet. 2008;371(9626):1783-9.

148. Lindstrom J, Peltonen M, Eriksson JG, llanne-Parikka P, Aunola S, KeinanenKiukaanniemi S, Uusitupa M, Tuomilehto J, Finnish Diabetes Prevention S. Improved lifestyle and decreased diabetes risk over 13 years: long-term follow-up of the randomised Finnish Diabetes Prevention Study (DPS). Diabetologia. 2013;56(2):28493.

149. Knowler WC, Fowler SE, Hamman RF, Christophi CA, Hoffman HJ, Brenneman AT, Brown-Friday JO, Goldberg R, Venditti E, Nathan DM. 10-year follow-up of diabetes incidence and weight loss in the Diabetes Prevention Program Outcomes Study. Lancet. 2009;374(9702):1677-86. 
150. Roumen C, Blaak EE, Corpeleijn E. Lifestyle intervention for prevention of diabetes: determinants of success for future implementation. Nutr Rev. 2009;67(3):132-46.

151. Torjesen PA, Birkeland KI, Anderssen SA, Hjermann I, Holme I, Urdal P. Lifestyle changes may reverse development of the insulin resistance syndrome. The Oslo Diet and Exercise Study: a randomized trial. Diabetes Care. 1997;20(1):26-31.

152. Chin SH, Kahathuduwa CN, Binks M. Physical activity and obesity: what we know and what we need to know. Obes Rev. 2016;17(12):1226-44.

153. Blaak EE. Carbohydrate quantity and quality and cardio-metabolic risk. Curr Opin Clin Nutr Metab Care. 2016;19(4):289-93.

154. Lin X, Zhang X, Guo J, Roberts CK, McKenzie S, Wu WC, Liu S, Song Y. Effects of Exercise Training on Cardiorespiratory Fitness and Biomarkers of Cardiometabolic Health: A Systematic Review and Meta-Analysis of Randomized Controlled Trials. J Am Heart Assoc. 2015;4(7).

155. Cassidy S, Thoma C, Houghton D, Trenell MI. High-intensity interval training: a review of its impact on glucose control and cardiometabolic health. Diabetologia. 2017;60(1):7-23.

156. Caspersen CJ, Powell KE, Christenson GM. Physical activity, exercise, and physical fitness: definitions and distinctions for health-related research. Public Health Rep. 1985;100(2):126-31.

157. Thompson D, Karpe F, Lafontan M, Frayn K. Physical activity and exercise in the regulation of human adipose tissue physiology. Physiol Rev. 2012;92(1):157-91.

158. Stanford KI, Middelbeek RJ, Goodyear LJ. Exercise Effects on White Adipose Tissue: Beiging and Metabolic Adaptations. Diabetes. 2015;64(7):2361-8.

159. Stanford KI, Goodyear LJ. Exercise regulation of adipose tissue. Adipocyte. 2016;5(2):153-62.

160. Hodgetts V, Coppack SW, Frayn KN, Hockaday TD. Factors controlling fat mobilization from human subcutaneous adipose tissue during exercise. J Appl Physiol (1985). 1991;71(2):445-51.

161. Romijn JA, Coyle EF, Sidossis LS, Gastaldelli A, Horowitz JF, Endert E, Wolfe RR. Regulation of endogenous fat and carbohydrate metabolism in relation to exercise intensity and duration. Am J Physiol. 1993;265(3 Pt 1):E380-91.

162. Karpe F, Fielding BA, llic V, Humphreys SM, Frayn KN. Monitoring adipose tissue blood flow in man: a comparison between the (133)xenon washout method and microdialysis. Int J Obes Relat Metab Disord. 2002;26(1):1-5.

163. Brenner BM, Ballermann BJ, Gunning ME, Zeidel ML. Diverse biological actions of atrial natriuretic peptide. Physiol Rev. 1990;70(3):665-99.

164. Moro C, Pillard F, De Glisezinski I, Harant I, Rivi??Re D, Stich V, Lafontan MAX, Crampes FO, Berlan M. Training Enhances ANP Lipid-Mobilizing Action in Adipose Tissue of Overweight Men. Medicine \& Science in Sports \& Exercise. 2005;37(7):1126-32.

165. Samra JS, Simpson EJ, Clark ML, Forster CD, Humphreys SM, Macdonald IA, Frayn $\mathrm{KN}$. Effects of epinephrine infusion on adipose tissue: interactions between blood flow and lipid metabolism. Am J Physiol. 1996;271(5 Pt 1):E834-9.

166. Berggren JR, Hulver MW, Houmard JA. Fat as an endocrine organ: influence of exercise. J Appl Physiol (1985). 2005;99(2):757-64.

167. Mulla NA, Simonsen L, Bulow J. Post-exercise adipose tissue and skeletal muscle lipid metabolism in humans: the effects of exercise intensity. J Physiol. 2000;524 Pt 3:919-28.

168. Moro C, Pillard F, de Glisezinski I, Crampes F, Thalamas C, Harant I, Marques MA, Lafontan M, Berlan M. Sex differences in lipolysis-regulating mechanisms in overweight subjects: effect of exercise intensity. Obesity (Silver Spring). 2007;15(9):2245-55. 
169. Stallknecht B, Kiens B, Helge JW, Richter EA, Galbo H. Interstitial glycerol concentrations in human skeletal muscle and adipose tissue during graded exercise. Acta Physiol Scand. 2004;180(4):367-77.

170. Ryden M, Jocken J, van Harmelen V, Dicker A, Hoffstedt J, Wiren M, Blomqvist L, Mairal A, Langin D, Blaak E, Arner P. Comparative studies of the role of hormonesensitive lipase and adipose triglyceride lipase in human fat cell lipolysis. Am J Physiol Endocrinol Metab. 2007;292(6):E1847-55.

171. Jocken JW, Blaak EE. Catecholamine-induced lipolysis in adipose tissue and skeletal muscle in obesity. Physiol Behav. 2008;94(2):219-30.

172. Mauriege P, Despres JP, Prud'homme D, Pouliot MC, Marcotte M, Tremblay A, Bouchard C. Regional variation in adipose tissue lipolysis in lean and obese men. $J$ Lipid Res. 1991;32(10):1625-33.

173. Reynisdottir S, Wahrenberg H, Carlstrom K, Rossner S, Arner P. Catecholamine resistance in fat cells of women with upper-body obesity due to decreased expression of beta 2-adrenoceptors. Diabetologia. 1994;37(4):428-35.

174. Crampes F, Beauville M, Riviere D, Garrigues M. Effect of physical training in humans on the response of isolated fat cells to epinephrine. J Appl Physiol (1985). 1986;61(1):25-9.

175. Crampes F, Riviere D, Beauville M, Marceron M, Garrigues M. Lipolytic response of adipocytes to epinephrine in sedentary and exercise-trained subjects: sex-related differences. Eur J Appl Physiol Occup Physiol. 1989;59(4):249-55.

176. Riviere D, Crampes F, Beauville M, Garrigues M. Lipolytic response of fat cells to catecholamines in sedentary and exercise-trained women. J Appl Physiol (1985). 1989;66(1):330-5.

177. Despres JP, Bouchard C, Savard R, Prud'homme D, Bukowiecki L, Theriault G. Adaptive changes to training in adipose tissue lipolysis are genotype dependent. Int $\mathrm{J}$ Obes. 1984;8(1):87-95.

178. Despres JP, Bouchard C, Savard R, Tremblay A, Marcotte M, Theriault G. The effect of a 20-week endurance training program on adipose-tissue morphology and lipolysis in men and women. Metabolism. 1984;33(3):235-9.

179. De Glisezinski I, Crampes F, Harant I, Berlan M, Hejnova J, Langin D, Riviere D, Stich V. Endurance training changes in lipolytic responsiveness of obese adipose tissue. Am J Physiol. 1998;275(6 Pt 1):E951-6.

180. van Aggel-Leijssen DP, Saris WH, Wagenmakers AJ, Senden JM, van Baak MA. Effect of exercise training at different intensities on fat metabolism of obese men. $J$ Appl Physiol (1985). 2002;92(3):1300-9.

181. Richterova B, Stich V, Moro C, Polak J, Klimcakova E, Majercik M, Harant I, Viguerie $\mathrm{N}$, Crampes F, Langin D, Lafontan M, Berlan M. Effect of endurance training on adrenergic control of lipolysis in adipose tissue of obese women. J Clin Endocrinol Metab. 2004;89(3):1325-31.

182. de Glisezinski I, Moro C, Pillard F, Marion-Latard F, Harant I, Meste M, Berlan M, Crampes F, Riviere D. Aerobic training improves exercise-induced lipolysis in SCAT and lipid utilization in overweight men. Am $J$ Physiol Endocrinol Metab. 2003;285(5):E984-90.

183. Stich V, de Glisezinski I, Galitzky J, Hejnova J, Crampes F, Riviere D, Berlan M. Endurance training increases the beta-adrenergic lipolytic response in subcutaneous adipose tissue in obese subjects. Int J Obes Relat Metab Disord. 1999;23(4):374-81.

184. Sutherland LN, Bomhof MR, Capozzi LC, Basaraba SA, Wright DC. Exercise and adrenaline increase PGC-1\{alpha\} mRNA expression in rat adipose tissue. J Physiol. 2009;587(Pt 7):1607-17.

185. Trevellin E, Scorzeto M, Olivieri M, Granzotto M, Valerio A, Tedesco L, Fabris R, Serra R, Quarta M, Reggiani C, Nisoli E, Vettor R. Exercise training induces 
mitochondrial biogenesis and glucose uptake in subcutaneous adipose tissue through eNOS-dependent mechanisms. Diabetes. 2014;63(8):2800-11.

186. Stallknecht B, Vinten J, Ploug T, Galbo H. Increased activities of mitochondrial enzymes in white adipose tissue in trained rats. Am J Physiol. 1991;261(3 Pt 1):E410-4.

187. Stanford KI, Middelbeek RJ, Townsend KL, Lee MY, Takahashi H, So K, Hitchcox KM, Markan KR, Hellbach K, Hirshman MF, Tseng YH, Goodyear LJ. A novel role for subcutaneous adipose tissue in exercise-induced improvements in glucose homeostasis. Diabetes. 2015;64(6):2002-14.

188. Vernochet C, Mourier A, Bezy O, Macotela Y, Boucher J, Rardin MJ, An D, Lee KY, Ilkayeva OR, Zingaretti CM, Emanuelli B, Smyth G, Cinti S, Newgard CB, Gibson BW, Larsson NG, Kahn CR. Adipose-specific deletion of TFAM increases mitochondrial oxidation and protects mice against obesity and insulin resistance. Cell Metab. 2012;16(6):765-76.

189. Bostrom P, Wu J, Jedrychowski MP, Korde A, Ye L, Lo JC, Rasbach KA, Bostrom EA, Choi JH, Long JZ, Kajimura S, Zingaretti MC, Vind BF, Tu H, Cinti S, Hojlund K, Gygi SP, Spiegelman BM. A PGC1-alpha-dependent myokine that drives brown-fatlike development of white fat and thermogenesis. Nature. 2012;481(7382):463-8.

190. Cao L, Choi EY, Liu X, Martin A, Wang C, Xu X, During MJ. White to brown fat phenotypic switch induced by genetic and environmental activation of a hypothalamic-adipocyte axis. Cell Metab. 2011;14(3):324-38.

191. Ruschke K, Fishbein L, Dietrich A, Kloting N, Tonjes A, Oberbach A, Fasshauer M, Jenkner J, Schon MR, Stumvoll M, Bluher M, Mantzoros CS. Gene expression of PPARgamma and PGC-1alpha in human omental and subcutaneous adipose tissues is related to insulin resistance markers and mediates beneficial effects of physical training. Eur J Endocrinol. 2010;162(3):515-23.

192. Ronn T, Volkov P, Tornberg A, Elgzyri T, Hansson O, Eriksson KF, Groop L, Ling C. Extensive changes in the transcriptional profile of human adipose tissue including genes involved in oxidative phosphorylation after a 6-month exercise intervention. Acta Physiol (Oxf). 2014;211(1):188-200.

193. Roberts R, Hodson L, Dennis AL, Neville MJ, Humphreys SM, Harnden KE, Micklem KJ, Frayn KN. Markers of de novo lipogenesis in adipose tissue: associations with small adipocytes and insulin sensitivity in humans. Diabetologia. 2009;52(5):882-90.

194. Skurk T, Alberti-Huber C, Herder C, Hauner H. Relationship between adipocyte size and adipokine expression and secretion. J Clin Endocrinol Metab. 2007;92(3):102333.

195. Zachwieja JJ, Hendry SL, Smith SR, Harris RB. Voluntary wheel running decreases adipose tissue mass and expression of leptin mRNA in Osborne-Mendel rats. Diabetes. 1997;46(7):1159-66.

196. Bradley RL, Jeon JY, Liu FF, Maratos-Flier E. Voluntary exercise improves insulin sensitivity and adipose tissue inflammation in diet-induced obese mice. Am J Physiol Endocrinol Metab. 2008;295(3):E586-94.

197. Frydelund-Larsen L, Akerstrom T, Nielsen S, Keller P, Keller C, Pedersen BK. Visfatin mRNA expression in human subcutaneous adipose tissue is regulated by exercise. Am J Physiol Endocrinol Metab. 2007;292(1):E24-31.

198. Holmes AG, Watt MJ, Febbraio MA. Suppressing lipolysis increases interleukin-6 at rest and during prolonged moderate-intensity exercise in humans. J Appl Physiol (1985). 2004;97(2):689-96.

199. Keller C, Keller P, Marshal S, Pedersen BK. IL-6 gene expression in human adipose tissue in response to exercise--effect of carbohydrate ingestion. $J$ Physiol. 2003;550(Pt 3):927-31.

200. Bluher M, Williams CJ, Kloting N, Hsi A, Ruschke K, Oberbach A, Fasshauer M, Berndt J, Schon MR, Wolk A, Stumvoll M, Mantzoros CS. Gene expression of 
adiponectin receptors in human visceral and subcutaneous adipose tissue is related to insulin resistance and metabolic parameters and is altered in response to physical training. Diabetes Care. 2007;30(12):3110-5.

201. Sjogren P, Sierra-Johnson J, Kallings LV, Cederholm T, Kolak M, Halldin M, Brismar $\mathrm{K}$, de Faire U, Hellenius ML, Fisher RM. Functional changes in adipose tissue in a randomised controlled trial of physical activity. Lipids Health Dis. 2012;11:80.

202. Trachta P, Drapalova J, Kavalkova P, Touskova V, Cinkajzlova A, Lacinova Z, Matoulek M, Zelinka T, Widimsky J, Jr., Mraz M, Haluzik M. Three months of regular aerobic exercise in patients with obesity improve systemic subclinical inflammation without major influence on blood pressure and endocrine production of subcutaneous fat. Physiol Res. 2014;63 Suppl 2:S299-308.

203. Moghadasi M, Mohebbi H, Rahmani-Nia F, Hassan-Nia S, Noroozi H, Pirooznia N. High-intensity endurance training improves adiponectin mRNA and plasma concentrations. Eur J Appl Physiol. 2012;112(4):1207-14.

204. Klimcakova E, Polak J, Moro C, Hejnova J, Majercik M, Viguerie N, Berlan M, Langin $D$, Stich V. Dynamic strength training improves insulin sensitivity without altering plasma levels and gene expression of adipokines in subcutaneous adipose tissue in obese men. J Clin Endocrinol Metab. 2006;91(12):5107-12.

205. Polak J, Klimcakova E, Moro C, Viguerie N, Berlan M, Hejnova J, Richterova B, Kraus I, Langin D, Stich V. Effect of aerobic training on plasma levels and subcutaneous abdominal adipose tissue gene expression of adiponectin, leptin, interleukin 6, and tumor necrosis factor alpha in obese women. Metabolism. 2006;55(10):1375-81.

206. Hulver MW, Zheng D, Tanner CJ, Houmard JA, Kraus WE, Slentz CA, Sinha MK, Pories WJ, MacDonald KG, Dohm GL. Adiponectin is not altered with exercise training despite enhanced insulin action. Am $J$ Physiol Endocrinol Metab. 2002;283(4):E861-5. 
CHAPTER 2 


\section{Targeting fatty acid metabolism to improve glucose metabolism}

Stinkens R., Goossens G.H., Jocken J.W., Blaak E.E.

Obesity Reviews, 2015; 16(9):715-57 


\begin{abstract}
Disturbances in fatty acid metabolism in adipose tissue, liver, skeletal muscle, gut and pancreas play an important role in the development of insulin resistance, impaired glucose metabolism and type 2 diabetes mellitus. Alterations in diet composition may contribute to prevent and/or reverse these disturbances through modulation of fatty acid metabolism.

Besides an increased fat mass, adipose tissue dysfunction, characterized by an altered capacity to store lipids and an altered secretion of adipokines, may result in lipid overflow, systemic inflammation and excessive lipid accumulation in nonadipose tissues like liver, skeletal muscle and the pancreas. These impairments together promote the development of impaired glucose metabolism, insulin resistance and type 2 diabetes mellitus. Furthermore, intrinsic functional impairments in either of these organs may contribute to lipotoxicity and insulin resistance. The present review provides an overview of fatty acid metabolismrelated pathways in adipose tissue, liver, skeletal muscle, pancreas and gut, which can be targeted by diet or food components, thereby improving glucose metabolism.
\end{abstract}




\section{INTRODUCTION}

Obesity is considered a global health problem, since it is closely associated with the development of chronic metabolic diseases, including cardiovascular disease, type 2 diabetes mellitus (T2D) and certain types of cancer [1]. The pathogenesis of T2D is characterized by the development of both insulin resistance in peripheral tissues and pancreatic $\beta$-cell failure [2, 3]. Disturbances in fatty acid metabolism play a crucial role in the development of an impaired glucose metabolism and diabetes. Combined dietary and physical activity intervention may reduce the incidence of T2D by $30-60 \%[4,5]$, which may for a considerable part be explained through modulation of fatty acid metabolism [6]. A better understanding of the interaction between diet, fatty acid metabolism, insulin resistance and $\beta$-cell dysfunction is needed to develop novel strategies to prevent impairments in glucose metabolism and, consequently, the development of T2D.

A tight interplay between adipose tissue, skeletal muscle, liver, pancreas and the gut regulate fatty acid metabolism in the human body. Besides an increased fat mass, adipose tissue dysfunction, characterized by an altered capacity to store lipids and low-grade inflammation, plays a major role in the development of insulin resistance and impaired glucose metabolism by promoting excessive fat storage in non-adipose tissues like liver, skeletal muscle, pancreas, the heart and kidneys [710] (Figure 1).

Furthermore, intrinsic functional impairments in either of these organs may contribute to lipotoxicity and insulin resistance. Skeletal muscle is generally considered the most important organ in peripheral insulin resistance [11]. Beside an increased fatty acid (FA) supply [12], a reduced skeletal muscle oxidative capacity $[13,14]$ may contribute to the accumulation of triacylglycerol (TAG) and bioactive lipid metabolites [15-17]. An increased lipid supply to the liver may result in an increased hepatic TAG content and very low-density lipoprotein (VLDL-TAG) output [18], a higher glucose production and output [19-21] and a reduced insulin clearance by the liver [22-24], leading to hyperlipidemia, insulin resistance and glucose intolerance [25]. Furthermore, hyperglycemia may, together with hyperlipidemia-related lipotoxicity in the pancreas, result in decreased glucosestimulated insulin secretion by the pancreatic $\beta$-cells [26].

Recent evidence also indicates that the gut microbiota and its products may contribute to the development of insulin resistance and a disturbed glucose metabolism (Figure 1). There is accumulating evidence that alterations in the gut microbiota composition and function may affect adipose tissue, liver and skeletal muscle lipid and glucose metabolism, at least partly through effects on bile acid metabolism [27-29].

Energy intake and diet composition (i.e. dietary fatty acids, polyphenols, fibers) may have a significant impact on many aspects of fatty acid metabolism in different tissues. These effects can be both acute (i.e. postprandial phase) and more longterm, ultimately affecting health status. The aim of the present review is to provide an overview of fatty acid metabolism related pathways in adipose tissue, liver, skeletal muscle, pancreas and gut that can be targeted by diet or food components and, as a consequence, improve whole-body glucose metabolism and insulin sensitivity. The focus of the present review will be on the major tissues involved in inter-organ substrate metabolism and lipid-induced insulin resistance. Associations 
between an increased myocardial triglyceride content or epicardial fat volume with insulin resistance, T2D and cardiac dysfunction [30-32], and the beneficial effects of diet on these deleterious parameters have been described elsewehere [33-35]. In addition, recent evidence points out that lipid accumulation in the kidneys [36, 37] and bones [38-40] may contribute to disturbances in whole-body glucose metabolism. These findings may be promising areas of future research but are beyond the scope of the present review. 


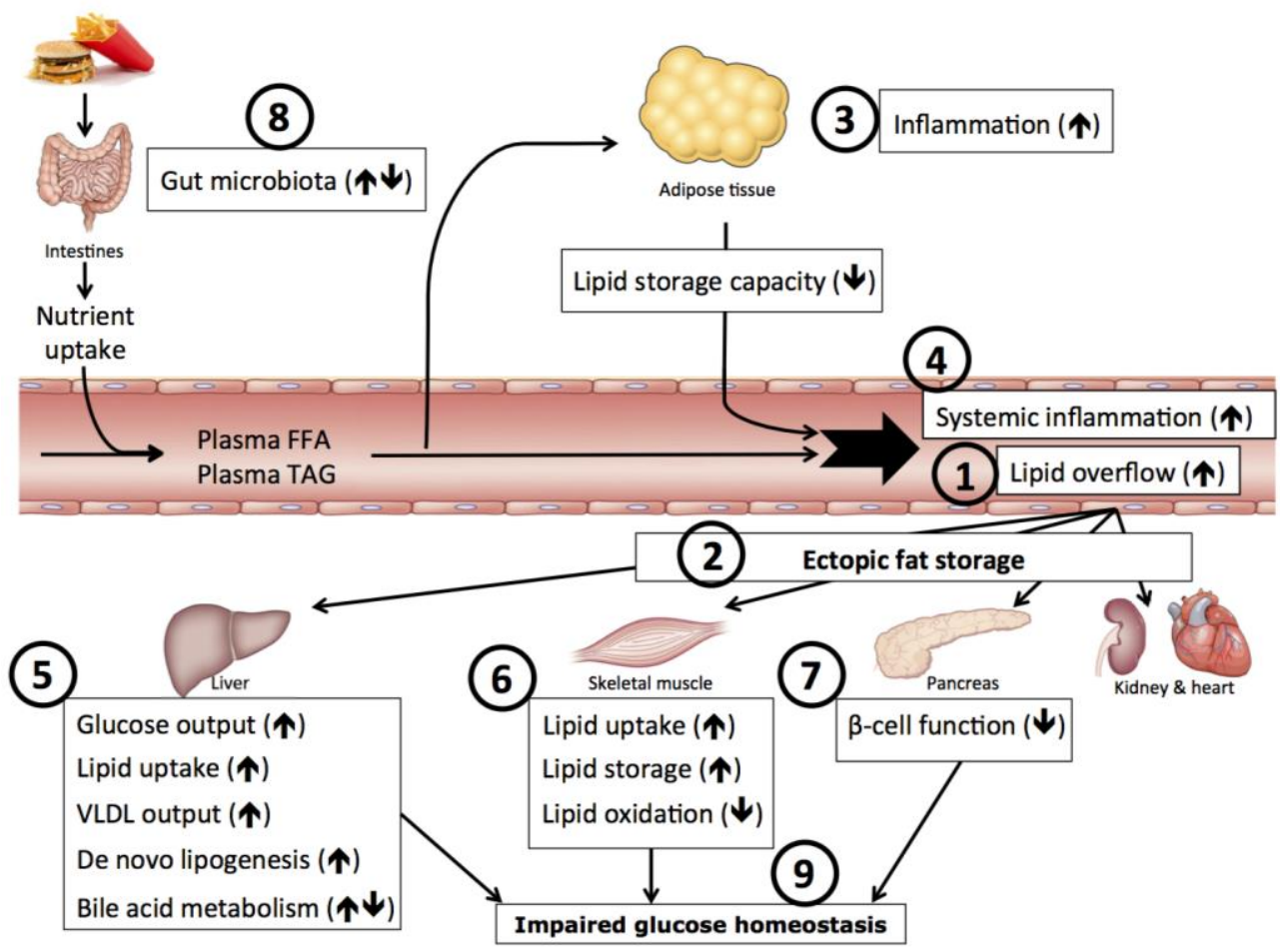

Figure 1. Inter-organ crosstalk in fatty acid metabolism and insulin resistance

An impaired adipose tissue lipid metabolism, as observed in obesity, is characterized by a decreased lipid storage capacity, which contributes to lipid overflow in the circulation (1), resulting in excessive fat storage in peripheral tissues such as skeletal muscle, liver, pancreas, kidney and heart (ectopic fat storage) (2). Furthermore, adipose tissue dysfunction is characterized by an altered expression and secretion of adipokines, inducing a state of chronic low-grade inflammation (3). This inflammatory state may on the one hand affect local adipose tissue lipid metabolism and on the other hand contribute to systemic inflammation (4), which together may affect lipid handling in peripheral tissues such as liver and skeletal muscle and promote insulin resistance through interference with insulin signaling. An increased lipid supply to the liver may result in a higher glucose production, an increased hepatic TAG content and VLDL-TAG output and a reduced insulin clearance by the liver (5). The increased insulin concentration stimulates de novo lipogenesis and, together with an increased VLDL-TAG output, results in hypertriglyceridemia, ultimately leading to insulin resistance and glucose intolerance. In skeletal muscle, besides an increased fatty acid supply and uptake, an impaired muscle lipid turnover may contribute to accumulation of TAG and bioactive lipid metabolites (DAG, LCFA-CoA and ceramides), which may interfere with insulin signaling (6). Hyperglycemia may, together with the formation of hyperlipidemia-related toxic metabolites and lipid accumulation in the pancreas, result in decreased glucose-stimulated insulin secretion by the pancreatic $\beta$-cells (7). Finally, alterations in gut microbiota composition and function (8) may affect adipose tissue, liver and skeletal muscle lipid and glucose metabolism, possibly through effects on SCFA production and bile acid metabolism. Ectopic fat storage is associated with impaired function of the liver, skeletal muscle and pancreas, leading to derangements in whole-body glucose homeostasis and, consequently, type 2 diabetes (9).

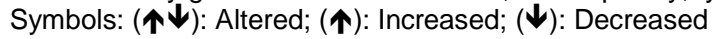




\section{ADIPOSE TISSUE DYSFUNCTION AND IMPAIRED GLUCOSE METABOLISM}

Adipose tissue is highly important in buffering the daily influx of dietary lipids. The buffering capacity of adipose tissue refers both to its ability to suppress the release of free fatty acids (FFA) into the circulation and to increase lipid uptake, which is most pronounced in the postprandial state [41].

Firstly, disturbances in the adipose tissue lipid buffering capacity, like impairments in lipid uptake, lipolysis and fatty acid storage, adipocyte differentiation, adipose tissue expandability and adipose tissue mitochondrial function will be discussed. Subsequently, the browning of white adipose tissue (WAT) in relation to thermogenesis will be adressed. Thirdly, lipid-induced inflammation in relation to impaired glucose metabolism will be described, as depicted in Figure 2. Finally, putative nutritional targets that may improve adipose tissue dysfunction will be addressed.

\section{Lipid uptake in adipose tissue}

In the postprandial period, VLDL- and chylomicron-TAG are hydrolyzed in the process of intravascular lipolysis by lipoprotein lipase (LPL) [42]. The FFA that are liberated by this process can be taken up by the adipose tissue but a significant proportion will also spillover in the circulation and enter the plasma FFA pool [43]. LPL is regulated in a tissue-specific manner by nutrients and hormones [44]. In adipose tissue, the major activator of LPL activity is insulin [45, 46]. Therefore, adipose tissue LPL activity is high in the fed state and low when fasted [47, 48].

Furthermore, research has shown that dexamethasone (a synthetic glucocorticoid) decreased in vitro and in vivo LPL mRNA expression and activity in isolated rat and mice adipose cells and tissue, resulting in increased circulating TAG concentrations [49, 50]. In contrast, dexamethasone increased LPL mRNA and activity in human adipose tissue cultures $[51,52]$. Whether these differences in LPL response reflect species-specific differences remains unclear. Another feedingrelated hormone that could be responsible for changes in LPL expression and activity is glucose-dependent insulinotropic polypeptide (GIP), which increases LPL activity and mRNA in isolated human and rodent adipocytes [53,54], although the regulation of adipose LPL activity by GIP in vivo remains to be established.

Recent studies also showed that LPL activity is altered by glycosylphosphatidylinositol-anchored high-density lipoprotein-binding protein 1 (GPIHBP1), which binds LPL [55] in the interstitial space and transports it to the capillary lumen across endothelial cells [56, 57]. Fasting and peroxisome proliferator-activated receptor (PPAR)-y agonists increased GPIHBP1 expression in rodents, while PPAR- $\alpha$ and PPAR- $\delta$ agonists had little or no effect [58]. Furthermore, hyperglycemia altered GPIHBP1 expression and activity in rodent and bovine endothelial cells [59]. Whether nutritional status or PPAR- $\gamma$ agonists alter GPIHBP1 expression in humans is currently unknown, but several GPIHBP1 mutations have been discovered in humans [60] and result in severe hypertriglyceridemia [55, 56, 61]. Therefore, modulation of GPIHBP1 expression or activity might provide a strategy to treat hypertriglyceridemia, cardiovascular disease and T2D. 
A considerable part of the variation in LPL activity during fasting, exercise and in the postprandial state may be explained by post-translational regulation by apolipoproteins (APO-C1, -2, -3, -5, APO-E) and the angiopoietin-like proteins (ANGPTL-3, -4, -8), as reviewed in detail elsewhere [62]. ANGPTL4, in particular, has been identified as an inhibitor of LPL activity and may be regulated by diet and fasting, since its expression and secretion changes in parallel with circulating FFA concentrations [62-66].

In obese conditions, basal LPL activity has been reported to be increased, whereas FA spillover from LPL-mediated TAG hydrolysis across adipose tissue during hyperinsulinemia is less suppressed as compared to lean healthy individuals [67, 68]. Indeed, the removal of TAG across adipose tissue was found to be impaired in obesity, insulin resistance and T2D, due to a reduced insulin-mediated stimulation of LPL activity [68-73], suggesting less efficient removal of dietary lipids by adipose tissue in these subjects. In line, a recent study has demonstrated that the relative quantity of meal fat stored in adipose tissue after the intake of subsequent meals was significantly reduced in (abdominally) obese versus lean individuals [69], which in turn may contribute to increased lipid spillover and ectopic fat deposition. To define nutritional targets affecting the regulation of LPL activity, which may subsequently reduce lipid spillover, ectopic fat deposition and improve insulin sensitivity, additional studies to determine the role of different LPL modulators in the regulation of its activity in vivo are clearly needed.

In addition to impaired LPL action in obese and insulin resistant conditions, disturbances in the uptake of the liberated FFAs (lipid spillover) may contribute to impaired lipid buffering. FAs are able to cross the endothelial barrier via passive diffusion, which is dependent on the concentration gradient of FAs across the membrane of the adipocyte, or via active transport [41, 43, 74, 75]. Active FA transport is facilitated by several enzymes and proteins, of which numerous FAtransport proteins have been identified in adipocytes [76], including fatty acid translocase/CD36 (CD36), membrane-bound and cytosolic fatty acid binding protein (FABPpm and FABPc, respectively) and fatty acid transporter protein (FATP) [77]. It has been shown that the translocation of CD36 from the intracellular stores to the plasma membrane is regulated by various stimuli, including insulin and increased activation of AMP-activated protein kinase (AMPK) [78-81]. Although CD36 protein expression is higher in the subcutaneous adipose tissue of obese, overweight and T2D compared to lean subjects [82], the net removal of TAG and FAs by adipose tissue is reduced in the former groups, suggestive of impairments in intracellular CD36 trafficking. Taken together, strategies to reduce spillover and increase FFA trapping in adipose tissue, mainly in the postprandial phase, will lead to a reduced lipid spillover and may consequently prevent or at least reduce ectopic fat deposition. On the one hand, this may be achieved by an increased FFA concentration gradient across the adipocyte membrane and a subsequent decreased intracellular FFA concentration. This might be accomplished by increasing FA reesterification and/or oxidation, which will be discussed later in this section. On the other hand, an increased facilitated diffusion by fatty acid transporters like CD36 may possibly increase fatty acid trapping, thereby reducing FFA spillover. Indeed, it has been shown that tissue-specific CD36 transcripts differentially influence fatty acid homeostasis and insulin sensitivity [83]. Adipocyte CD36 appears to be metabolically protective, and its selective upregulation might 
have therapeutic potential in insulin resistance. Nevertheless, it has been shown that CD36 only facilitates fatty acid transport in adipose tissue and muscle when extracellular concentrations are low, suggesting no major regulatory role when circulating FFA concentrations are equal or above those seen in the overnight postabsorptive state [84]. 


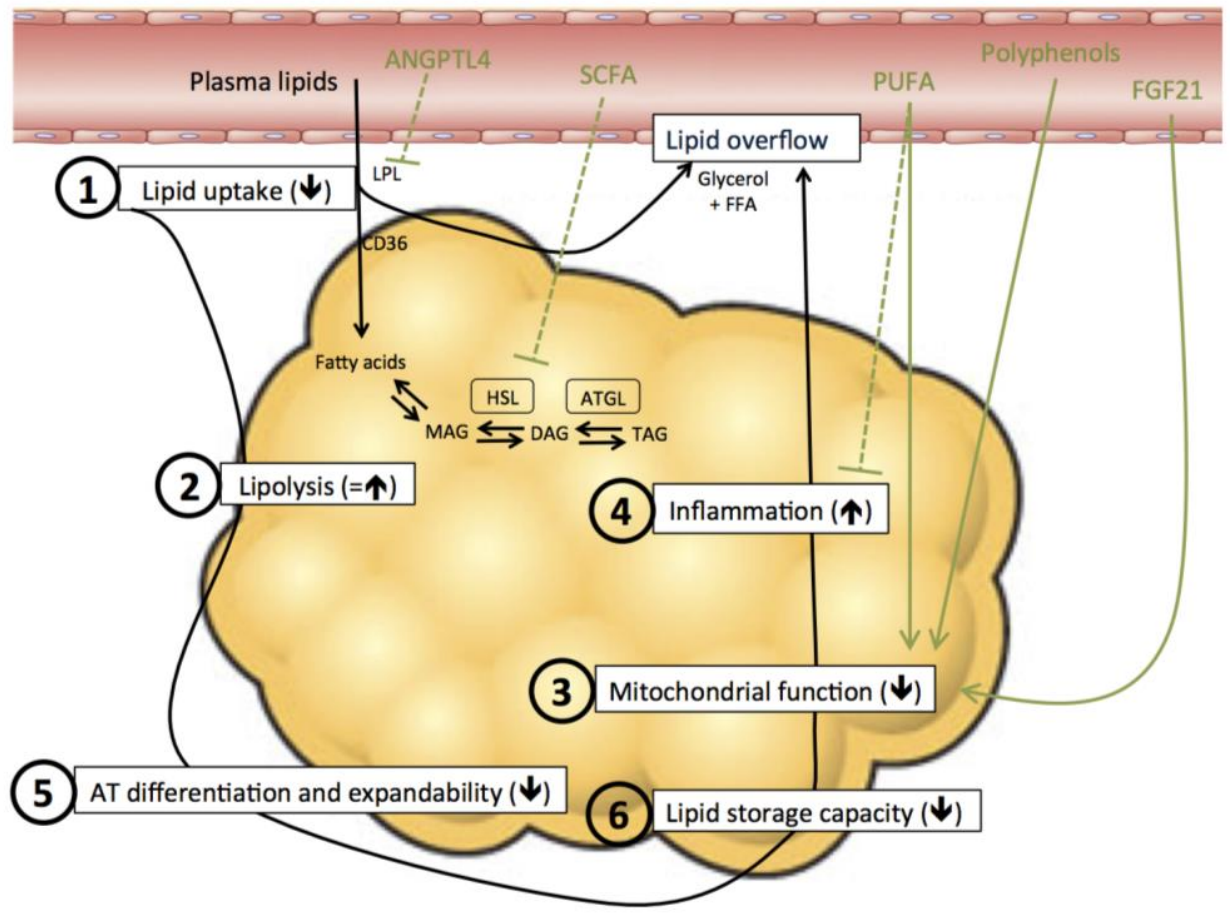

Figure 2. Disturbances in adipose tissue fatty acid metabolism

Adipose tissue lipid uptake (1) is impaired in obesity, insulin resistance and T2D, due to a reduced insulin-mediated stimulation of LPL activity and, possibly, decreased facilitated FFA uptake via CD36 in adipocytes, which contributes to lipid overflow in the circulation. TAG and DAG are broken down by the lipases ATGL and HSL. Fasting lipolysis, expressed per unit fat mass, may be decreased in obese subjects (2). Nevertheless, insulin-mediated suppression of adipose tissue lipolysis per unit fat mass is attenuated in obese individuals, resulting in an increase in whole-body lipolysis during postprandial conditions. A decreased adipose tissue mitochondrial function (3) may lead to impaired scavenging of fatty acids, resulting in increased intracellular FFA concentrations, reducing FFA trapping and an enhanced release of FFA into the circulation. Furthermore, dynamic changes occur in the adipose tissue immune cell populations during the development of obesity, causing a shift from an antiinflammatory towards a more pro-inflammatory phenotype, resulting in a state of low-grade inflammation (4), affecting insulin sensitivity through different mechanisms. Finally, a reduced adipose tissue differentiation and expandability (5), leading to a reduced lipid storage capacity (6), contributes to lipid overflow into the circulation.

Abbreviations: LPL: Lipoprotein lipase; FFA: Free fatty acid; CD36: Fatty acid translocase CD36; TAG: Triacylglycerol; DAG: Diacylglycerol; MAG: monoacylglycerol; ATGL: Adipose triglyceride lipase; HSL: Hormone sensitive lipase; ANGPTL4: Angiopoietin-like protein 4; PUFA: Poly unsaturated fatty acid; SCFA: Short chain fatty acid; FGF21: Fibroblast growth factor 21.

Dashed lines indicate inhibition. Solid lines indicate stimulation. Green lines indicate beneficial effects. Symbols: (=): unchanged; $(\boldsymbol{\uparrow})$ : Increased; $(\boldsymbol{\downarrow})$ : Decreased. 


\section{Fatty acid release from adipose tissue}

When energy is required (e.g. during exercise and fasting), the lipids that have been stored in intracellular lipid droplets (LD's) as TAG, are hydrolyzed via activation of the intracellular lipolytic pathway, which involves different lipases and lipid droplet-associated proteins that are under hormonal control [85].

Catecholamines and natriuretic peptides (NPs) act as lipolytic hormones, while insulin acts as the major anti-lipolytic hormone in human adipose tissue. The net lipolytic effect of catecholamines is determined by the ratio between lipolytic beta $(\beta 1,2$ and 3)- and anti-lipolytic alpha ( $\alpha 2)$-receptors, and subsequent protein kinase A (PKA) activation. NPs act through natriuretic peptide receptor type $A, B$ and $C$ (NPRA, NPRB, NPRC) to increase protein kinase G (PKG) activity [86-88]. Stimulation of intracellular lipolysis is dependent on PKA and PKG-mediated phosphorylation of LD-associated proteins, including perilipin 1 (PLIN-1), hormonesensitive lipase (HSL) and adipose triglyceride lipase (ATGL), as extensively reviewed elsewhere [89, 90].

Whole-body lipolysis under fasting conditions (basal lipolysis) may be increased in obesity because of the increased total adipose tissue mass. However, if adipose tissue would release FFA at the same rate in obese and lean subjects, then circulating FFA would be much higher than observed in obesity (only 20-30\% higher), suggesting that FFA concentrations are not elevated in proportion to fat mass in obese individuals. Indeed, others and we have demonstrated that fasting lipolysis expressed per unit fat mass is rather reduced in obesity [91-93]. This was accompanied by downregulation of the expression of the key lipolytic enzymes HSL and ATGL $[91,94,95]$. Data on PLIN-1 are inconclusive, being reduced [9698] or even elevated [99] in obese adipose tissue. Since humans are in the postprandial state most of the day, insulin-mediated inhibition of adipose tissue lipolysis is a major regulator of lipolytic rate. Insulin-mediated suppression of adipose tissue lipolysis per unit fat mass is attenuated in obese individuals, suggesting that chronic hyperinsulinemia cannot overcome the increase in wholebody lipolysis. Therefore, inhibition of adipose tissue lipolysis might be a therapeutic strategy to limit excess FFA release, thereby alleviating the development of insulin resistance and obesity-associated metabolic abnormalities. Although data are scarce, reduced plasma FFA and glucose levels have been demonstrated in diabetic rats treated with a selective HSL inhibitor for 3-8 hours [100]. Recently, Girousse et al. [101] have shown that mice treated with the same pharmacological HSL inhibitor for 7 days and haploinsufficient $\mathrm{HSL}^{+/-}$mice were paradoxically resistant to diet-induced obesity due to a reduction in FFA uptake and reesterification, suggestive of reshaping the FFA flux in peripheral tissues [101]. Furthermore, in that study, systemic glucose tolerance was improved through induction of de novo lipogenesis in murine and human adipocytes. In addition to selective inhibition of HSL, recent data report about the development of a selective inhibitor of ATGL, Atglistatin [102], and about the mechanisms of inhibition of ATGL by long-chain acyl-coenzyme A [103], highlighting the increasing interest in selective lipase inhibition to correct defects in lipid metabolism for the treatment and prevention of obesity and obesity-associated metabolic diseases.

In contrast to an elevated basal lipolysis, others and we have clearly shown that in vitro and in vivo catecholamine-induced lipolysis is blunted in subcutaneous 
adipose tissue of obese subjects, which persists after significant weight loss [89, $94,95]$. These data suggest that impaired cathecholamine-induced lipolysis may be an important primary factor in the development of obesity. Data are limited with respect to NP-induced lipolysis. However, reduced circulating NP levels [104] and a defective ANP-mediated lipolytic response in subcutaneous adipocytes and adipose tissue from obese subjects have been observed [105]. Therefore, in contrast to the anti-lipolytic approach with selective lipase inhibition, improving catecholamine- and NP-sensitivity has been extensively investigated for the treatment of obesity and obesity-related complications but has, so far, not shown promising results due to cardiovascular side effects and receptor desensitization [106-108].

In addition to classical lipolysis, three recent studies have implicated autophagy, a homeostatic mechanism functioning as a 'self-digestion' system, in selective lipid hydrolysis under basal and catecholamine-stimulated conditions in adipocytes, termed lipophagy [109-111]. These data indicate that activation of cytosolic lipases (i.e. ATGL and HSL) is no longer the sole molecular mechanism to liberate FA from adipocyte TAG stores. Since ATGL and HSL are expressed at much higher level in adipocytes than other cell types it is plausible that under normal physiological conditions adipocytes rely mainly on classical cytosolic lipolysis, while the alternative pathway for lipid breakdown, lipophagy, may become more important in pathophysiological conditions, with a reduced ATGL and HSL activity, to maintain lipid homeostasis. In line, autophagy markers and fluxes appear to be elevated in adipose tissue of obese insulin resistant and T2D subjects [112-115]. In addition, nutritional and hormonal regulation of adipose tissue autophagy is impaired in obese rodents [116].

To summarize, obesity is characterized by an increased basal and a blunted catecholamine and NP-stimulated lipolysis in subcutaneous adipocytes. This altered lipid turnover may be an early factor in the development of increased fat stores and obesity-associated metabolic complications. Modulation of classical lipolysis recently regained interest in the treatment of obesity-related insulin resistance, indicated by the development of selective ATGL and HSL inhibitors. However, to prevent excessive gain in body weight, tissue FFA turnover (uptake, esterification and oxidation) should be adapted accordingly. Furthermore, lipohagy might be increased in adipose tissue of obese subjects as compensatory mechanism to deal with the increased lipid availability due to an attenuated classical lipolysis. Importantly, before considering manipulation of the classical and/or alternative pathway of adipose tissue lipolysis for therapeutic purposes, a better insight into its role in pathophysiology as well as hormonal and nutritional regulation is warranted.

\section{Lipid droplet formation and fatty acid storage in the adipose tissue}

In humans, adipocytes, the major cell type in white and brown adipose tissue (BAT), are specialized for storing lipids in LD. Several proteins bind the LD surface and regulate LD size, fusion and number. They include PAT proteins (i.e. perilipin 1, perilipin 2/adipophilin/ADRP, perilipin 3/TIP47 and perilipin 4/S3-12), CIDE (Cell Death inducing DNA Fragmentation Factor) proteins, Soluble NSF Attachment 
Protein Receptor (SNARE), caveolins and several lipases. LDs are dynamic organelles, constantly forming, growing or shrinking. In recent years, our knowledge of LD dynamics and biogenesis has increased, as reviewed extensively elsewhere [117-123]. Impaired LD expansion and TAG storage capacity may play a role in lipid storage diseases, including obesity and T2D. In obese conditions, the storage capacity of the LD in adipocytes is exceeded. CIDE-C/FSP27 (fat-specific protein 27) and PLIN-1 largely regulate TAG storage in human adipocytes by facilitating lipid transfer from smaller to larger LDs and by regulating intracellular lipolysis. Studies examining the expression of CIDE proteins and PLIN-1 as a function of insulin sensitivity found that mRNA levels of these LD-associated proteins correlate positively with insulin sensitivity in subjects with similar body mass index $[124,125]$. These findings in humans contrast with findings in mice, in which the lack of CIDEC/FSP27 or PLIN-1 protected against high fat diet-induced obesity and insulin resistance [126-128], highlighting the difficulties in extrapolating results from mice to human pathologies.

In summary, although human data are limited, high levels of protein that promote TAG storage, including CIDEC/FSP27 and PLIN-1, might help to sequester lipids in the adipose tissue and to protect against insulin resistance. Furthermore, impaired LD expandability may prevent the recruitment of new adipocytes by either initiating a pro-inflammatory response or by preventing the secretion of yet unidentified factors that promotes recruitment of adipocyte progenitors.

\section{Adipocyte differentiation and adipose tissue expandability}

A unique property of adipose tissue is its capacity to change its dimensions. This can be achieved by fat cell enlargement (hypertrophy) or by recruitment of new adipocytes from the resident pool of progenitor cells (hyperplasia).

During periods of chronic excessive energy intake, the adipose tissue mass expands first by hypertrophy to the point that the maximal fat cell expandability is achieved. Then, signals are released by the adipocyte to stimulate proliferation and/or differentiation of preadipocytes (adipogenesis) [129], leading to an increased number of mature adipocytes. This process is necessary to store the excessively available FAs and to protect cells from detrimental effects of high concentrations of circulating FAs [130]. Interestingly, there seems to be adipose tissue depot-specific patterns in adipose tissue expansion. Overfeeding evokes adipocyte hypertrophy in abdominal subcutaneous adipose tissue, whereas adipocyte hyperplasia, but not hypertrophy, occurs in the femoral subcutaneous fat [131]. Adipogenesis is also accompanied by specific changes in the adipocyte extracellular matrix (ECM), as reviewed elsewhere [132-134], and this ECM remodeling seems essential in adipose tissue expansion. Research showed that ECM processes are disrupted in obesity and impair metabolic function and fat mass expansion [135, 136]. Furthermore, diet induced weight gain by overfeeding, resulted in fat mass expansion and upregulation of genes involved in lipid metabolism and storage, angiogenesis and ECM remoddeling [137].

The inability of the adipose tissue to store excess lipids in newly differentiated adipocytes results in enlargement of existing adipocytes [138]. These hypertrophic adipocytes are less sensitive to the action of insulin and have impaired lipid 
buffering capacity [139]. The adipogenic potential of adipose-derived stem cells (ASCs) depends on their depot specific origin and host characteristics such as age, sex and metabolic status [140]. ASCs from subcutaneous fat have been reported to differentiate better into mature adipocytes than those from visceral fat [141]. In contrast to what was thought in the past, the expandability of the adipose tissue is not an unlimited process. Importantly, impaired adipose tissue expandability has been linked to metabolic derangements. For example, lipodystrophic patients with severely reduced adipose tissue mass, show a substantial reduction in fat storage capacity. Hypertrophic adipocytes in obese individuals, on the other hand, are overloaded with stored TAG. In both conditions, the storage capacity of adipose tissue seems insufficient, resulting in ectopic fat storage and, consequently, insulin resistance [7].

Kim et al. [138] have shown that ectopic fat storage and insulin resistance can be ameliorated when adipose tissue mass can properly expand to accommodate excess calories. Involvement of ASCs in the impaired adipose tissue expandability is only beginning to be explored. It has been reported that the number of mature adipocytes is set during childhood and stays constant throughout adulthood regardless of fat mass changes in humans [142], but it is not yet clear how adipose progenitor cells contribute to this process. Thus, strategies to increase adipose tissue expandability may reduce ectopic fat storage, thereby improving insulin sensitivity, despite possible body weight gain.

\section{Adipose tissue mitochondrial function and browning}

Mitochondria play a central role in the catabolism of nutrients to provide energy that is required for numerous cell functions. During periods of energy requirement, FFAs are liberated to fuel mitochondrial $\beta$-oxidation. There is substantial evidence that adipose tissue mitochondrial mass and function determine metabolic health. Rodent studies have suggested that mitochondria in white adipose tissue contribute to overall fat oxidation [143]. Impaired mitochondrial oxygen consumption in adipose tissue is present in mouse models of obesity and T2D [144], and mitochondrial oxidative pathways are downregulated in human adipose tissue [145]. Importantly, adequate mitochondrial function is essential to maintain adipose tissue function, glucose homeostasis [146] and protects against insulin resistance and T2D [144]. This is further highlighted by a recently large-scale microarray analysis using $>1000$ human abdominal subcutaneous adipose tissue biopsies, revealing that mitochondrial oxidative pathways were markedly downregulated, whereas inflammatory pathways were upregulated, in morbidly obese insulin resistant as compared to morbidly obese insulin sensitive patients [147]. In accordance, mitochondrial mass and electron transport chain genes in adipose tissue are lower in obese and T2D subjects [148, 149]. A recent study has demonstrated that both human adipocyte mitochondrial content and ex vivo respiration (oxygen consumption) were significantly reduced in obese as compared to lean individuals, independent of adipocyte size [150]. A decreased adipose tissue mitochondrial function may lead to an incorrect scavenging of fatty acids, resulting, on the one hand, in increased intracellular FFA concentrations reducing FFA trapping and, on the other hand an enhanced release of FFA, liberated by 
intravascular or intracellular lipolysis into the circulation, contributing to lipid overflow [151]. Interestingly, the 'browning' of white adipocytes, which will be further discussed below, likely involves lipase-generated PPAR ligands, whereby lipolysis may be an initiating factor (e.g. recruiting adult adipocyte progenitor cells into a brown adipocyte lineage) [152].

Traditionally, fat cells have been divided into unilocular white and multilocular brown adipocytes. Brown adipocytes are specialized heat producing cells, and transfer energy from lipid and carbohydrate substrates into heat through the actions of uncoupling protein-1 (UCP-1). In contrast to infants, who have substantial amounts of BAT to maintain body temperature, it was thought for many years that healthy adult humans living under normal environmental temperatures lack BAT. However, ${ }^{18}$ F-fluoro-D-2-deoxy-D-glucose (FDG) positron emission tomography (PET)-positive areas have recently been identified in lean healthy adults, indicating that substantial amounts of BAT are present in humans [153155]. Furthermore, it has been demonstrated that BAT is inversely related to body fat percentage and BMI [153], and BAT activity was increased 1 year after bariatric surgery-induced weight loss in morbidly obese subjects [156]. Recently, BAT has been implicated to improve whole body glucose homeostasis and insulin sensitivity in humans [157]. Moreover, a third category of fat cells has been introduced, namely "beige/brite" adipocytes, which have a brown fat-like morphology within white fat depots [158-161]. Beige adipocytes seem to be programmed to be bifunctional, meaning they are suitable for energy storage in the absence of thermogenic stimuli but capable of switching on heat production [158]. It is not entirely clear yet what distinguishes brown from beige adipocytes, and the relative importance of BAT in energy homeostasis also requires further investigation. Nevertheless, it has been estimated that BAT may contribute to $3-5 \%$ of basal metabolic rate in humans [162]. Therefore, an intriguing question is how BAT can be activated and whether this may induce weight loss in humans.

In addition to cold-induced BAT activity, several factors have been studied that affect activity and recruitment of brown adipocytes. Briefly, these include adrenergic stimulation, natriuretic peptides, irisin, capsinoids, and insulin in humans [162, 163]. In rodents, it has been suggested that, in addition to the abovementioned factors, thyroid hormone, bile acids, fibroblast growth factor 21 (FGF21) $[164,165]$ and bone morphogenetic proteins (BMP) [166] may also play a role. Future studies will likely elucidate whether strategies to increase browning of white adipose tissue hold promise to prevent and/or treat obesity and related impairments in lipid and glucose metabolism.

\section{Lipid-induced inflammation and impaired glucose metabolism}

Adipose tissue used to be considered as a passive fat storage organ. However, it is clear for more than two decades that adipose tissue is an active endocrine organ by the release of a variety of lipids (lipokines) [167] and signaling molecules (adipokines). These factors can both act locally (autocrine and/or paracrine) and at the whole-body level (endocrine function) to exert a wide range of biological effects and to regulate systemic metabolic homeostasis [7]. The endocrine function of adipose tissue is impaired in obese, insulin resistant individuals, which may 
contribute to chronic low-grade inflammation and, consequently, obesity-related insulin resistance and other complications [7]. In addition to adipocytes, adipose tissue contains a wide variety of stromal vascular cells, including endothelial cells, fibroblasts, preadipocytes and immune cells. Although mature adipocytes make up $>90 \%$ of adipose tissue volume, they account for only $20-40 \%$ of the cellular content. Interestingly, immune cells present in adipose tissue have recently been shown to play an important role in adipose tissue biology [164]. The low-grade inflammatory state in obesity is the result of an imbalance between the production of pro-inflammatory and anti-inflammatory factors. It was shown about 10 years ago that many proinflammatory factors are produced by macrophages that infiltrate hypertrophic adipose tissue $[168,169]$. More recent evidence indicates that also other innate and adaptive immune cells are present in adipose tissue, as reviewed elsewhere [170]. Furthermore, it seems that dynamic changes occur in the adipose tissue immune cell populations during the development of obesity, causing a shift from an anti-inflammatory towards a more pro-inflammatory phenotype. It has been demonstrated that there is a relative abundance of M1 polarized macrophages, Treg cells and eosinophils in lean visceral adipose tissue, whereas the proportion of M2 polarized macrophages, Th1 cells, mast cells, dendritic cell, neutrophils and natural killer $\mathrm{T}$ cells is increased in obesity. Intriguingly, many of the latter proinflammatory immune cells have been linked to impairments in glucose homeostasis $[171,172]$ and decreasing the inflammatory status may improve insulin resistance in rodents and humans [173].

An important but still unanswered question is what triggers adipose tissue inflammation in obesity? Lipids act as important signal moieties regulating both metabolism and immune responses. This is exemplified by the induction of hepatic and peripheral insulin resistance [174] and pro-inflammatory responses in adipose tissue [175] by lipids. Various mechanisms seem to be involved in the induction and progression of inflammatory responses in adipose tissue and consequently insulin resistance in obesity. Briefly, lipid-induced activation of innate receptors (Toll-Like Receptors (TLR)) and the inflammasome, death of hypertrophic adipocytes and resulting macrophage infiltration, lipid-induced endoplasmic reticulum stress (ER stress) and the unfolded protein response and, more recently, the gut microbiota have been linked to adipose inflammation and consequently insulin resistance in obesity [176]. On the other hand, activation of PPAR- $y$ by polyunsaturated fatty acids (PUFA) [177] may reduce adipose tissue inflammation [178]. However, it remains to be established to what extent the above-mentioned mechanisms contribute to systemic inflammation and whole-body insulin resistance in humans. Over the last decade, evidence has emerged that ER stress in several organs, including adipose tissue, plays a direct (i.e. as a negative modulator of the insulin signaling pathway) and indirect role (i.e. by promoting lipid accumulation) in the onset of insulin resistance, as reviewed elsewhere [179].

Adipose tissue inflammation may in turn exert detrimental effects on insulin sensitivity through different mechanisms. For example, tumor necrosis factor alpha (TNF- $\alpha$ ) and Interleukin 6 (IL-6) inhibit adipocyte differentiation [180-182] and TNFa induces adipocyte apoptosis in pre- and mature adipocytes [183], which could lead to the enlargement of the remaining fat cells and consequently, reduced adipose tissue lipid buffering. Furthermore, TNF- $\alpha$ and IL-6 both stimulate adipocyte lipolysis [184-187], thereby contributing to systemic FA release, which 
may in turn lead to ectopic fat storage and insulin resistance [188]. In addition, lipid-induced impairments in adipokine secretion may also exert direct effects on peripheral insulin sensitivity [189]. It has been demonstrated that adiponectin increases skeletal muscle fat oxidation and therefore, the decreased adiponectin concentrations in obese conditions may impact fat oxidation, and as such, affect lipid accumulation and insulin sensitivity [190, 191].

As reviewed by Ohashi et al [192], several anti-inflammatory adipokines, such as adiponectin, the family of the C1q/TNF-related Proteins (CTRP3, -6, -9), adipolin and omentin-1 may exert beneficial effects on obesity-related complications. CTRP3 is a novel adipokine, expressed at the AT and found in circulating plasma [193, 194], which regulates hepatic glucose output [195], suppresses chemokine production in response to lauric acid, LPS or TLR stimulation in macrophages and adipocytes [196] and stimulates the expression of adiponectin in primary human adipocytes and cultured 3T3-L1 adipocytes [197]. CTRP6 has been found to increase the expression of the anti-inflammatory cytokine IL-10 in human monocyte-derived macrophages [198], to stimulate activation of AMPK and enhance fatty acid oxidation in skeletal muscle cells [199]. In cultured myocytes, CTRP9 activates phosphorylation of AMPK and protein kinase B (PKB) and promotes insulin stimulated glucose uptake [194]. It also reduced diet-induced weight gain, decreased insulin resistance and hepatic steatosis, with enhanced AMPK activation and fat oxidation in skeletal muscle in CTRP9 transgenic mice [200]. CTRP12, also known as Adipolin (adipose-derived insulin-sensitizing factor) [201], has been reported to activate insulin signaling in the liver and adipose tissue of obese mice [202]. Furthermore, omentin-1, also known as intelectin-1, is abundantly expressed in visceral fat tissue [203], is decreased in obese individuals [204] and increased insulin stimulated glucose uptake in cultured adipocytes in vitro [203].

In addition, dipeptidyl peptidase-4 (DPP4) is released by mature adipocytes and inhibits skeletal muscle insulin signaling [205, 206]. Beside lipolysis and insulin signaling, pro-inflammatory cytokines may also regulate adipocyte mitochondrial function [207]. Of note, not only adipose tissue but also other organs such as the liver, skeletal muscle, heart and pancreas may contribute to lipid-induced systemic inflammation in obesity via secretion of cytokines [208, 209].

Taken together, lipids are important triggers for adipose tissue inflammation and consequently insulin resistance in obesity. Therefore, interventions aimed at improving lipid metabolism may improve glucose metabolism via reduction of adipose tissue and systemic inflammation.

\section{Putative nutritional targets to improve adipose tissue function}

The balance between lipolysis, adipocyte differentiation and mitochondrial function within adipose tissue is important to maintain adequate lipid storage capacity of adipose tissue, thereby preventing lipid overflow in the circulation and ectopic fat deposition. Therefore, improving the lipid buffering capacity of adipose tissue has high potential to increase glucose tolerance and insulin sensitivity. 
Presently, there is a clear need for additional studies to determine the regulators of LPL activity, including the involvement of proteins affecting post-translational mechanisms, like ANGPTL4, which may be regulated by diet [63].

Secondly, as discussed above partial HSL or ATGL inhibition may reduce lipid overflow and possibly improve metabolic profile. Interestingly, intravenous acetate infusion [210] or colonic acetate administration may reduce systemic FFA concentrations, through an effect on HSL phosphorylation [211]. Dietary manipulation of colonic acetate and short chain fatty acid (SCFA) concentration through pre- and probiotics may be a promising target in this respect.

Thirdly, modulation of the adipose tissue lipophagic pathway might be a potential target pathway as well. Recently, we have shown that dietary polyphenols including resveratrol and epigallocatechin-gallate (EGCG), found naturally in red wine and green tea have caloric restriction-like effects in overweight humans [212]. Interestingly, our microarray data showed that an improved adipose tissue lipolysis and function, is associated with selectively targeting of the master regulator of lipophagy, TFEB, in human adipose tissue following resveratrol supplementation [213]. However, it remains to be determined whether lipophagy-mediated lipid catabolism in adipose tissue is directly involved in the potential beneficial effects of polyphenols.

As indicated above, nutritional strategies to improve mitochondrial function, like specific polyphenols or a combination of polyphenols, may be effective in balancing lipid supply to utilization [212, 213], improving thereby adipose tissue function. Moreover, recent data from our group also suggest that a dysbalance between oxygen supply and oxygen utilization leading to an increased adipose tissue oxygen tension may induced adipose tissue dysfunction [214, 215], again illustrating the importance of a normal mitochondrial mass and function.

Factors affecting the activity and recruitment of BAT, may have positive effect with respect to adipose tissue function, body weight control and insulin sensitivity. Currently, most promising may be dietary components affecting our microbial bile acid metabolism, leading to increased circulating FGF21, a mediator of lipid and carbohydrate metabolism, also inducing recruitment of brown adipocytes [216].

The effect of dietary quality on adipose tissue function and ectopic fat accumulation remains an area of particular interest in composing optimal diets that minimize ectopic and abdominal fat accumulation. There is strong evidence that avoidance of high saturated fatty acids (SFA) diet contributes to lower health risks among obese, metabolic syndrome and diabetic patients [217]. There are indications that the monounsaturated fatty acids (MUFA) and/or PUFA have more beneficial effects compared to SFA on the action of insulin [218-221]. Research showed that $n-3$ PUFA (eicosapentaenoic acid (EPA), docosapentaenoic acid (DPA) and docosahexaenoic acid (DHA)) reduced LD formation in 3T3-L1 cells compared to SFA [222]. Further, long chain n-3 PUFA may increase fatty acid oxidation and mitochondrial biogenesis in adipose tissue [223, 224], may inhibit fat cell proliferation [225] and may limit fat cell hypertrophy and hyperplasia [226]. Additionally, positive effect have been ascribed to specifically $n-6$ fatty acids in reducing abdominal fat area, improving insulin sensitivity and in reducing visceral fat/subcutaneous fat ratio compared with a SFA diet [227, 228].

Dietary fat quality not only modulates lipid metabolism, it may also affect low-grade inflammation, as for instance seen by exposure of myotubes or adipocytes to SFA 
which increased IL-6 mRNA and protein expression, possibly via activation of nuclear factor-kB (NF-kB) [229, 230]. A human intervention study with a SFA rich diet for 5 weeks showed higher concentrations of C-reactive protein (CRP), fibrinogen and IL-6 compared to a diet enriched in MUFA [231]. Substantial increase in the PUFA intake can also decrease low-grade inflammation as indicated by circulating CRP, IL-6 or soluble adhesion molecules [232, 233].

In summary, polyphenols, specific dietary fatty acids and pre- and probiotics may be promising nutritional components in improving the balance between lipolysis, autophagy and mitochondrial function and in stimulation of adipose tissue browning, thereby improving adipose tissue function and whole-body glucose homeostasis. For an overview of putative nutritional targets see Table 1.

\section{ALTERED LIVER FUNCTION AND IMPAIRED GLUCOSE METABOLISM}

Obesity is recognized as a major cause of the promotion of metabolic diseases including non-alcoholic fatty liver disease (NAFLD), which is not only linked to an impaired glucose metabolism and diabetes, but also evokes more severe liver diseases like non-alcoholic steatohepatitis (NASH), hepatic cirrhosis and eventually liver cancer.

Indeed, hepatic insulin resistance, which is defined as an impaired suppression of hepatic glucose production [234], may originate from the accumulation of lipid metabolites that interfere with insulin signaling [235]. This accumulation of lipids and lipid metabolites may be caused by multiple factors [236]. Besides that, liver lipid accumulation is also associated with the progression of ER stress, mitochondrial stress and an impaired autophagy, resulting in lipotoxicity [237]. An increased FFA release from visceral adipose tissue (the 'portal hypothesis') or increased intake of dietary fat, followed by a reduction in postprandial lipid clearance and an increased spillover of FFA in the circulation, due to a reduced lipid storage capacity, can explain this phenomenon [238-241]. Furthermore, mitochondrial dysfunction, associated with insulin resistance may precede liver fat accumulation by impairing fatty acid $\beta$-oxidation [242]. Additionally, an increased de novo lipogenesis highly contributes to liver lipid accumulation, ultimately leading to steatosis [243] (Figure 3).

When FA supply to the liver is increased, VLDL-TAG secretion by the liver is also increased, but it appears that this secretion of VLDL-TAG is not sufficient to compensate for the increased uptake, resulting in a net lipid accumulation [244], even though conditions of insulin resistance are marked by an increased production of VLDL-TAG [245]. Below, the mechanisms contributing to liver fat accumulation are briefly delineated.

\section{Hepatic lipid uptake}

In obesity, insulin resistance and T2D, circulating levels of FFA and lipoprotein remnants are increased [246-248] and this causes an increased storage of TAG and lipid metabolites in hepatocytes and may lead to hepatic insulin resistance. This may further increase circulating VLDL-TAG concentrations due to a decreased insulin-mediated suppression of VLDL production by the liver [249-252]. 
In the liver, beside $\mathrm{LPL}$, hepatic lipase $(\mathrm{HL})$ plays an important role in fatty acid metabolism, as it is both a phospholipase and a TAG lipase [253]. An increased HL activity promotes FFA uptake into hepatocytes and has been shown to be increased in obese humans [254-256], insulin resistant rodents [257], type 1 diabetic patients [258] and in NAFLD patients [259].

The rate of plasma FFA uptake by the liver is determined by both the plasma FA concentration and the hepatocellular capacity for FA uptake [260], which depends on the number and activity of transporter proteins on the plasma membrane of the hepatocyte. The main proteins in the hepatic transmembrane FA transport are FATP-2 [261], FATP-5 [262], caveolins, and to a lower extent fatty acid translocase CD36 [263, 264]. FATP-5 knockout mice show resistance to diet-induced obesity and hepatic lipid accumulation, but there is no evidence for involvement of this isoform in human obesity [265]. Also, silencing of hepatic FATP-5 in hyperglycemic mice showed a rapid (2-3 weeks) reduction in liver FFA uptake and a reduction in serum glucose levels, reaching normal glycaemia after 5 weeks [262]. Additionally, expression of FABP-4 and -5 correlated with hepatic fatty acid infiltration in NAFLD patients [266]. Interestingly, it was shown that specific bile acids (BAs) (ursodeoxycholic acid (UDCA) and deoxycholic acid (DCA)) inhibit liver-specific FATP-5 in mice [267], suggesting that specific bile acids can impact hepatic lipid metabolism. Caveolin proteins consisting out of caveolin-1, -2 and -3 , play an important role in protein trafficking and the formation of lipid droplets. Caveolin-1 knockout mice have lower TAG accumulation in the liver and are resistant to the development of diet-induced obesity, suggesting that this protein may be of importance in liver fat accumulation and TAG synthesis [268]. Furthermore, rodent in vivo and in vitro data show that overexpression of caveolin-3 in liver resulted in an improved insulin receptor signaling, insulin sensitivity and glucose metabolism [269]. Normally, CD36 is not highly expressed in liver, but its expression was positively correlated with hepatic TAG content in NAFLD patients, underscoring the importance of this protein in lipid accumulation [270]. Moreover, CD36 deficiency increased insulin sensitivity in muscle, but resulted in an insulin resistant state in the liver of mice [271]. It is evident that further research is required to elucidate the role of these FA transporters in normal physiology as well as pathological conditions.

\section{Hepatic lipolysis}

Rodent in vivo and in vitro data showed that hepatic ATGL (also known as PNPLA2 and desnutrin) knockdown enhances glucose tolerance by increasing hepatic glucose utilization and improved insulin action from hepatic TAG accumulation [272]. Furthermore, HSL-knockout mice also showed reduced hepatic TAG stores and increased hepatic insulin sensitivity [273], but results are not consistent [274]. In adipose tissue, ATGL and HSL are highly expressed and active [275], but in the liver, the contribution of other lipases may also play a role in the catabolism of stored lipids. Several members of the carboxylesterase/lipase family and the patatin-like phospholipase domain-containing protein (PNPLA) family have been suggested as potential TAG hydrolases [276]. One of them, carboxyl esterase3/triglyceride hydrolase-1 (Ces-3/Tgh-1, ortholog of human Ces-1), has gained 
major interest because the recent characterization of Ces-3/Tgh-1-deficient mice provided compelling evidence that the enzyme participates in the assembly and secretion of hepatic VLDL [277]. PNPLA-4 and -5 exhibit TAG-hydrolase, DAGtransacylase and retinylester hydrolase activity in vitro [278], but whether these activities are also relevant in vivo remains to be determined [90]. The adiponutrin/PNPLA-3 I148M polymorphism was reported to be associated with insulin resistance and NAFLD, suggesting a role of importance in hepatic TAG accumulation [279-281]. Furthermore, LD-covering proteins also play a role in the pathophysiology in fatty liver disease, which is characterized by hepatocytes containing LD with excessive neutral lipids [282]. Studies showed that LD proteins such as PLIN-1 and PLIN-2 are highly expressed in liver steatosis [283, 284]. A high fat diet increases expression of PLIN-2 in a PPAR-y dependent manner, resulting in the development of fatty liver [285-287]. Data from a rodent study showed that PLIN-2 knockout enhanced insulin action in the liver, whereas muscle and adipose tissue were not affected [288]. Furthermore, other LD-associated proteins such as FSP27 and CIDEC have also been characterized. FSP27 was expressed in the steatoic liver of a T2D mouse model and the expression was markedly decreased in livers lacking PPAR-y. Forced expression of FSP27 in hepatocytes in vitro or in vivo led to an increase of LD through increased TAG levels, as reviewed elsewhere [289].

In addition to the classical lipolysis, recent evidence suggests a role for autophagy and lysosomal lipid degradation in liver LD (also termed lipophagy) [109, 290], via the action of lysosomal lipase (LAL) [109]. Even though this lipophagy accounts for a high percentage of the lipolysis that takes place in response to lipid challenges and during prolonged starvation in liver [109], also a certain percentage of degradation of lipids in lysosomes may occur continuously in many cell types (e.g. adipose tissue and skeletal muscle) [109]. Further research is warranted to determine the reasons behind the coexistence and possible co-regulation of the two different lipolytic pathways, the one mediated by the cytosolic lipases (i.e. ATGL and HSL) and the other mediated by the autophagic-lysosomal degradation system (i.e. $L A L)$. It is possible that activation of one lipolytic pathway or the other may be related to total capacity (i.e. lipophagy may be able to degrade larger amounts of triglycerides in shorter time). However, it is also possible that the quality and type of the resulting lipolytic products differs between cytosolic and lysosomal lipases, but data on this are largely lacking. Lastly, in light of the growing evidence in support of the heterogeneity of the cellular LD, it is also plausible that the two lipolytic systems target different subpopulations of LD's [291].

In the presence of insulin resistance and hyperinsulinemia, hepatic autophagy has been reported to be reduced [292] and it has been shown that defective autophagy is causal to impaired hepatic insulin sensitivity and glucose homeostasis in obese mice [293]. Since most evidence of autophagy comes from rodent studies, more research is warranted to elucidate the exact mechanism of action of hepatic autophagy and possible lysosomal dysfunction in human insulin resistant subjects. 


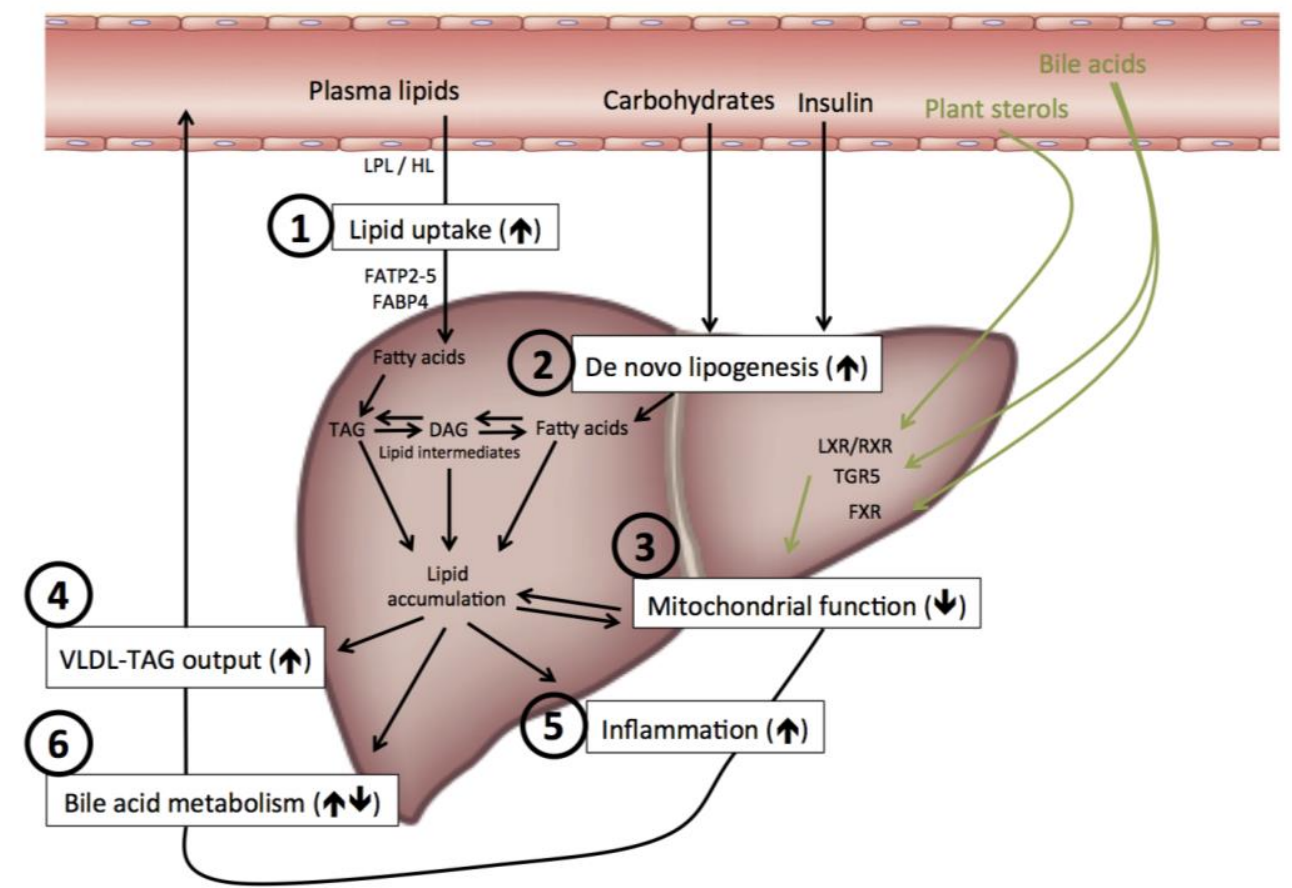

Figure 3. Disturbances in liver fatty acid metabolism

When circulating levels of FFA and lipoprotein remnants are increased, uptake and storage (1) of TAG and lipid metabolites in hepatocytes occur via increased LPL, HL activity and number and activity of transporter proteins (such as FATP2-5, FABP4) leading to hepatic insulin resistance. Increased insulinstimulated de novo lipogenesis (2) contributes to hepatic lipid accumulation. In addition, under insulin resistant conditions hepatic mitochondrial function is decreased (3) and may inhibit FA oxidation, contributing to hepatic lipid accumulation, increased VLDL production (4) and an inflammatory state (5). During conditions of hepatic lipid accumulation, an overproduction of VLDL occurs and together with an increased de novo lipogenesis results in hypertriglyceridemia, a condition often seen in insulin resistance and T2D. Finally, circulating bile acid composition (6) is altered in insulin resistant and T2D patients and might affect liver lipid metabolism via membrane (e.g. TGR5) and nuclear (e.g. RXR) receptor signaling. Interestingly, plant sterols have been shown to be effective nuclear receptor activators. Abbreviations: FFA: Free fatty acids; TAG: Triacylglycerol; DAG: Diacylglycerol; LPL: Lipoprotein lipase; HL: Hepatic lipase; FATP2-5: Fatty acid transport proteins 2-5; FABP4: Fatty acid binding protein 4; VLDL: Very-low-density lipoprotein; TGR5: G-protein coupled receptor TGR5; FXR: Farnesoid X receptor; LXR: Liver X receptor; RXR: Retinoid X receptor.

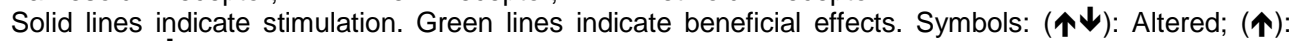
Increased; $(\boldsymbol{\downarrow})$ : Decreased 


\section{De novo lipogenesis}

On the other hand, increased insulin concentrations stimulate de novo lipogenesis (DNL), a process that is characterized by converting carbohydrates towards FAs and generating "new lipids" [294, 295]. The overproduction of VLDL together with an increased DNL, leads to hypertriglyceridemia, a condition often seen in insulin resistance and T2D [245]. During DNL, the conversion of glucose to fatty acids includes a coordinated series of enzymatic reactions of which fatty acid synthase (FAS) is the key rate-limiting enzyme that regulates the conversion of malonyl-CoA into palmitate, which is, thereafter, converted into complex fatty acids [296]. Knockout of FAS has been shown in mice to provoke a fatty liver phenotype upon high carbohydrate feeding, perhaps due to an increase in hepatic malonyl-CoA [297]. Another key enzyme is stearoyl-CoA desaturase 1 (SCD1), which plays a role in the desaturation of fatty acids and is predominantly expressed in liver. Depletion of SCD1 in mice showed a decreased lipogenesis and an increased $\beta$ oxidation [298]. During DNL, the final step of TAG synthesis is catalyzed by diacylglyceroltransferase (DGAT), which consists of DGAT1 and DGAT2. DGAT1 deficient mice are resistant to diet-induced obesity [299], have decreased levels of tissue TAG and are more insulin and leptin sensitive [300]. Furthermore, glucose and insulin regulate the expression of lipogenic enzymes via carbohydrate responsive element binding protein (ChREBP) [301] and sterol regulatory element binding protein-1 (SREBP1c) [302, 303], respectively. Both SREBP1c and ChREBP are involved in the transcriptional regulation of lipogenic genes and have been associated with increased DNL in NAFLD [304]. DNL is a highly regulated process and can lead to adverse metabolic consequences when dysregulated. Therapeutic targeting of this pathway may open a new window of opportunity for combating various lipogenesis-driven pathological conditions like obesity and insulin resistance.

\section{Hepatic mitochondrial function and fatty acid oxidation}

The majority of FAs are oxidized within mitochondria and lower amounts of oxidation takes place in peroxisomes [305], which are dynamic, multifunctional organelles that contribute to several anabolic and catabolic processes and are essential for human health and development (for review, see: [306, 307]). While medium- and short-chain FAs are thought to enter the mitochondrial matrix directly [308], long-chain FAs require carnitine palmitoyltransferase I (CPT-1) to enter the mitochondrial matrix. Insulin regulates CPT-1 by inhibiting transcription of the CPT1 gene [309] and this could lead to increased concentrations of TAG and lipid metabolites inside hepatocytes. In contrast, the peroxisomal $\beta$-oxidation system is CPT-1 independent, but complete FA oxidation does not take place [310] and the chain-shortened acyl-CoAs are consequently exported to the mitochondria where they undergo complete $\beta$-oxidation [311-313].

Research showed that during insulin resistant conditions, hepatic mitochondrial function is altered [314-316]. During insulin resistant conditions, an increased circulating glucose concentration may downregulate PPAR- $\alpha$ [317], which inhibits FA-oxidation in men [318] and contributes to hepatic lipid accumulation and increased VLDL production [319]. Furthermore, as reviewed by Koliaki et al. [320], 
an up-regulation of hepatic mitochondrial $\beta$-oxidation may occur as a result of lipid oversupply. This would stimulate hepatic ATP and reactive oxygen species production, possibly leading to local lipotoxicity, inflammation and oxidative stress and resulting in impairments in mitochondrial function and morphology. Since nondiabetic obese humans show normal or even greater hepatic mitochondrial function compared to lean humans, the authors suggest that hepatic energy metabolism transitionally adapts to chronic lipid overload by up-regulation of oxidative capacity in states of obesity and steatosis, which could be followed by a progressive decline in liver mitochondrial function during prolonged chronic insulin resistance, associated with T2D and NASH [320].

\section{Bile acid metabolism}

Once synthesized in the liver, the free bile acids (BAs) are conjugated with the amino acids taurine or glycine, secreted and stored in the gallbladder. After being released into the intestinal tract to facilitate the absorption of dietary fats and fatsoluble vitamins, about $95 \%$ of BAs are reabsorbed in the lateral ileum and transported back to the liver via the entero-hepatic circulation with only $5 \%$ of BAs excreted into the feces. The reabsorption of bile decreases the need for de novo bile acid synthesis [321, 322] and the loss of BAs in the feces represents the principal means of eliminating cholesterol from the body [321, 323, 324]. The synthesis of BAs by oxidation of cholesterol occurs exclusively in the liver and involves two different pathways. Firstly, the classical pathway converts cholesterol to 7a-hydroxyl-cholesterol to ultimately form 2 major primary BA products: chenodeoxycholic acid (CDCA) and cholic acid (CA) [325]. Secondly, the alternative pathway (the "acidic pathway") converts cholesterol to 27-hydroxycholesterol and produces mainly CDCA [326]. The intestinal microbiota metabolize primary BAs by dehydroxylation and deconjugation to form the secondary BAs lithocholic acid (LCA) and deoxycholic acid (DCA) [321, 323, 324]. The gut microbiota may not only regulate the conversion of $B A$, also the BA may regulate the microbiota composition [327]. Complementary to the aid of absorbing lipids and fat-soluble vitamins in the intestine, accumulating data also show that BAs have a signaling function in regulating biological processes by binding to the nuclear receptor farnesoid X receptor (FXR) and to the G-protein coupled receptor TGR5 (also known as GPR19) in gut and other tissues affecting glucose, lipid and energy metabolism [321, 323, 324], as discussed more extensively below.

\section{FXR-receptor}

The FXR-receptor is highly expressed in the liver, intestine, kidney and adrenal gland [321, 323, 324, 328] and is also shown to play a role in liver regeneration, inflammation and tumorgenesis [321, 324]. Both conjugated and unconjugated BAs can activate FXR and the order of potency is CDCA > LCA = DCA > CA. CA feeding increased hepatic expression of ApoC-2, a LPL activator, specifically through FXR as this effect was not observed in $\mathrm{FXR}^{-/-}$mice [329]. In addition, FXR activation increased the expression of VLDL receptor [330] and syndecan-1 [331], which are responsible for increased clearance of TAG-rich lipoprotein and remnant particles, respectively. Furthermore, BA sequestrants, such as cholestyramine, 
were found to increase plasma TAG, mediated at least in part, through FXR [332], which increased plasma lipoprotein clearance. However, other mechanisms may also be involved in FXR-mediated lipid lowering effects. For example, PPAR- $\alpha$ was induced by CDCA and GW4064 (a synthetic FXR agonist) treatment in HepG2 cells and primary hepatocytes [333]. GW4064 treatment also increased the mRNA expression of PDK4, a PPAR- $\alpha$ target gene involved in the regulation of substrate metabolism, in both rat hepatoma cells, human primary hepatocytes and also resulted in a reduced plasma TAG concentration in vivo in mice [334]. BA or GW4064 induced activation of FXR also increased the expression and secretion of FGF21 [335], a cytokine modulating systematic carbohydrate and lipid metabolism and reducing hepatic TAG levels [336-338]. FGF21 has been reported to inhibit lipogenesis through suppressing the transcriptional activity of SREBP-1c [339] and therefore, the FGF21 pathway may play an important role in FXR-mediated decrease in hepatic TAG levels, which may be of importance in decreasing hepatic insulin resistance [340, 341]. Besides the nuclear receptor $F X R$, the liver $X$ receptor (LXR- $\alpha$ and $L X R-\beta)$ and retinoid $x$ receptor $(R X R)$ play an important role in regulating carbohydrate and lipid metabolism in humans [342, 343]. Interestingly, sitosterol, campesterol and certain oxidized derivatives of phytosterols (oxyphytosterols) are effective LXR activators [344]. A rodent study showed that $L X R-\alpha$ and $L X R-\alpha / \beta$ knockout mice remained glucose tolerant and insulin sensitive, while LXR- $\beta$ knockout mice became highly insulin resistant after a high-fat diet [345]. Treatment of lean and ob/ob mice with the pharmacological LXR activator (GW3695) resulted in lower blood glucose levels and significantly improved whole body insulin sensitivity in the ob/ob mice, but no changes were found in the lean mice [346]. Furthermore, the synthetic LXR- $\alpha / \beta$ activator (T0901317) augmented diet-induced hyperlipidemia, normalized glucose tolerance and improved insulinstimulated glucose uptake in isolated soleus muscle and completely restored glucose transporter 4 (GLUT4) expression and insulin-stimulated AS160 phosphorylation in rat muscle [347]. Although the vast majority of studies have been performed in non-adipose cells/tissues, results in recent years suggest that LXRs may have important modulatory roles on adipose lipid and glucose metabolism [348].

In addition, PPAR- $\gamma$ and RXR agonists have complementary effects on glucose and lipid metabolism in human skeletal muscle [349], as well as in diabetic and obese rodent models [350]. It has also been shown that a RXR ligand (LG100754) improved insulin resistance in vivo in $\mathrm{db} / \mathrm{db}$ mice [351] and a recent study showed that a novel RXR partial agonist (CBt-PMN (11b)) has a glucose-lowering effect and improved insulin secretion and glucose tolerance in the liver of KK- $\mathrm{A}^{\mathrm{y}}$ mice [352].

\section{TGR5-receptor}

TGR5 is a family member of the G-protein coupled receptors (also known as GPR19) and is highly expressed in gallbladder, ileum and colon and in lower concentrations in BAT, liver, muscle and the central nervous system [336]. The activation of TGR5 is highest with the bile acid LCA, followed by DCA, CDCA and CA [323, 336] and results in the activation of PKA [336]. Furthermore, TGR5 activation regulates the expression of genes involved in inflammation [353], 
increases energy expenditure in skeletal muscle and BAT [354] through stimulation of mitochondrial function [355-357] and modulates plasma glucose and lipid concentrations [336, 355, 356, 358]. BA mediated activation of TGR5 has beneficial metabolic effects and the BA composition is altered in patients with insulin resistance [359] and T2D [360]. Since gram-positive bacteria have a more pronounced effect on the transformation of primary to secondary BAs [361, 362], compared to most of the gram-negative bacteria [363], an alteration in the gut microbiota might have a distinct effect on bile acid metabolism and might be an effective strategy to improve insulin sensitivity. Indeed, in the study of Vrieze et al., a vancomycin-induced decrease in gram-positive bacteria was associated with a reduced conversion of primary to secondary BAs and a tendency towards a reduced peripheral insulin sensitivity [364]. Also, transgenic liver overexpression of cholesterol 7a-hydroxylase, protected mice against high-fat diet induced obesity, fatty liver and insulin resistance [365]. Furthermore, glucose metabolism can also be improved in diabetic patients by administration of BA sequestrants, such as colesevelam [366, 367]. Thus, a promising nutritional strategy may be to alter the gut microbiota by modifying the diet by either prebiotics or probiotics and modulating thereby BA metabolism [368, 369].

\section{Hepatic inflammation}

Since insulin resistance and obesity are characterized by a low-grade inflammatory status [370], inflammation induced by the increased lipid accumulation, could also be an underlying cause for the development of hepatic insulin resistance. Of interest, ER stress has been shown to be involved in the development of hepatic inflammation and insulin resistance. First, ER stress can directly modify key hepatic enzymes involved in gluconeogenesis and lipogenesis, and stimulate stress kinases that interfere with insulin signaling. Secondly, ER stress may indirectly induce inflammation and lipotoxicity by promoting fat accumulation in hepatocytes [179, 371, 372].

Furthermore, hepatic peroxisomal FA oxidation causes an increased concentration of reactive oxygen species [373], which in excess cause a decrease in the natural antioxidant concentrations, leading to oxidative stress in hepatocytes [374, 375]. In turn, this oxidative stress causes hepatocyte degeneration and death [374] evoking an inflammatory response [376]. Specialized liver macrophages, called Kupffer cells, play a central role in this inflammatory process since in vitro stimulation of these cells by endotoxin (e.g. lipopolysaccharides (LPS)) or specific FAs (e.g. SFA) and their metabolites, lead to toll-like receptor signal transduction and the production of inflammatory cytokines, including TNF- $\alpha$ and IL-6 [377].

Furthermore, in analogy to the adipokines, the liver-derived proteins (known as hepatokines) [378] such as leukocyte cell-derived chemotaxin 2 (LECT2) regulate cross talk with other tissues and link obesity with skeletal muscle insulin resistance [379].

Taken together, the oversupply of lipids to the liver, together with impaired clearance of circulating remnants, increased de novo lipogenesis and decreased FA oxidation contribute to lipid accumulation in the liver, referred to as hepatic steatosis, which can progress to NAFLD, NASH and liver cancer [380]. 
Putative nutritional targets to reduce liver fat accumulation

All factors that improve adipose tissue function may reduce lipid overflow and spillover to the liver and will, as such, likely reduce hepatic steatosis.

Furthermore, it has been shown that BAs influence energy expenditure and glucose homeostasis via their effects on gluconeogenesis, insulin secretion and insulin sensitivity in both mouse and human studies [355, 358, 381]. BA-mediated activation of TGR5 has beneficial metabolic effects and the BA composition is altered in patients with insulin resistance. Secondary BAs can also affect host metabolism via binding to several nuclear receptors (FXR, LXR, RXR). Therefore, manipulating BA metabolism to increase concentrations of secondary BAs could be an attractive target to tackle obesity and insulin resistance $[358,381]$. An alteration of the gut microbiota might have distinct effects on BA metabolism and might therefore be an effective strategy to improve insulin sensitivity. Beside that, modulating gut microbiota may affect fermentation products from dietary fibers like SCFA and monosaccharides affecting thereby liver gluconeogenesis and lipogenesis, which might directly or indirectly affect glucose homeostasis [382].

The nuclear receptor FXR and LXR are important in regulating liver, muscle and adipose tissue lipid and glucose metabolism in humans and it has been shown that sitosterol, campesterol and certain oxidized derivatives of phytosterols (oxyphytosterols) are effective LXR activators [344].

In summary, in particular nutritional factors targeting BA metabolism, gut microbiota like pre and probiotics and factors affecting the nuclear receptors FXR and LXR like plant sterols seem promising targets in improving liver fat metabolism thereby improving glucose homeostasis (Table 1). 


\section{IMPAIRMENTS IN SKELETAL MUSCLE METABOLISM}

Accumulation of intramuscular TAG (IMTAG) has been associated with skeletal muscle insulin resistance in humans and is already present in young lean offspring of type 2 diabetic parents [12]. Both high fat diets and acute intravenous intralipid infusions result in increased IMTAG stores and a concomitant development of insulin resistance [383-386], highlighting an important role of lipid supply in fattyacid induced insulin resistance. Nevertheless, also several studies report similar IMTAG concentrations in obese insulin sensitive and obese T2D subjects [387, 388] indicating that intramuscular lipids are not directly linked to insulin resistance. Further evidence against a direct role of IMTAG in insulin resistance originates from the observation that endurance trained athletes also have high IMTAG levels and are highly insulin sensitive [389-391]. This so called 'athletes paradox' may be explained by a higher muscle oxidative capacity [389], a higher antioxidant capacity in the athletic group [392] or a higher concentration of intramuscular lipid droplets around the mitochondria after endurance exercise [393]. On the contrary, in obese, insulin resistant and T2D patients, an increased IMTAG concentration has been linked with a reduced oxidative capacity [394-398], which could lead to FA storage, rather than oxidation and thereby provide a mechanism for lipid accumulation within skeletal muscle (Figure 4). By now, it is obvious that the relationship between IMTAG accumulation and insulin sensitivity is not as straightforward as originally thought, but rather the accumulation of bioactive lipid metabolites like diacylglycerol (DAG), long chain fatty acyl-CoA and ceramides are involved in FA-induced insulin resistance [399-403]. Furthermore, others and we recently highlighted that, besides the amount and type of lipid metabolite, also the cellular localization is of major importance for the development of insulin resistance [404-406]. Although results are not consistent [407-411], it was recently described that skeletal muscle inflammation and macrophage markers are increased and associated with insulin resistance in rodents as well as humans and that treatment of high-fat-diet fed mice with the PPAR-y agonist rosiglitazone decreased muscle inflammation and improved local insulin signaling [412]. Also, several studies showed that muscle insulin resistance is associated with ER stress activation, as reviewed elsewhere [179, 413].

Taken together, it is obvious that the relationship between TAG or lipid metabolites is more complex as originally proposed and that understanding lipid turnover within skeletal muscle in addition to the role of increased lipid supply and uptake is crucial for elucidating the relationship between ectopic fat accumulation and insulin resistance. Therefore, the major components of skeletal muscle lipid turnover and intrinsic disturbances are discussed below.

\section{Skeletal muscle lipid uptake}

As mentioned before, there is mixed evidence for the notion that obesity is associated with increased fasting, postprandial, diurnal or nocturnal FFA concentrations. Instead, elevated circulating TAG concentrations in both the fasting and postprandial state may be far more striking associated with insulin resistance as compared to increased FFA concentrations. TAG concentrations have been reported to be increased in the obese insulin resistant state, which may be 
ascribed to an increased liver VLDL production [92] or to impairments in adipose tissue TAG clearance from the circulation [68-73].

In human studies, postprandial systemic TAG concentrations and TAG extraction across forearm muscle were significantly elevated in subjects with impaired glucose metabolism (either subjects with impaired fasting glucose (IFG) or impaired glucose tolerance (IGT)) versus normal glucose tolerant controls [414]. Another study comparing insulin resistant versus control subjects with the same stable isotope methodology could not confirm an increased postprandial muscle TAG extraction, despite elevated TAG concentrations [92]. Although the mixed results remain to be elucidated, this study also confirms the apparent importance of TAG metabolism in insulin resistance, showing that the insulin resistant state was closely related to increased TAG rather than increased FFA concentrations, most likely due to a partitioning towards TAG synthesis in the liver.

The expression and activation of muscle LPL plays a major role in skeletal muscle TAG extraction. It was shown that in mice LPL deletion reduces lipid storage and increases insulin signaling in skeletal muscle [415] and that muscle specific overexpression of LPL causes muscle-specific insulin resistance by causing defects in muscle signaling and action [416], whilst skeletal muscle LPL knockout mice show opposite effects. Whether these effects are translational to humans is currently unknown. Most studies indicate that fasting raises total LPL activity in human skeletal muscle [417, 418], but results are not consistent [44]. Furthermore, a single bout of physical exercise also leads to a marked increase in LPL activity, protein, and mRNA in the exercising muscle [417, 419-422]. In contrast to adipose tissue and liver, insulin infusion decreased skeletal muscle LPL activity in humans [423]. Additionally, muscle LPL activity is inhibited at posttranslational level by ANGPTL4 [424] but little is known about the physiological and molecular mechanisms involved in the regulation of muscle LPL expression. It has been shown that T2D patients have significantly lower plasma ANGPTL4 levels as compared to healthy subjects, thereby suggesting a role for ANGPTL4 in diabetes [63]. Furthermore, skeletal muscle ANGPTL4 expression is increased in human muscle following fasting via elevation of plasma FFA. However, the functional implications of fasting and lipid-induced ANGPTL4 expression in muscle as well as possible impairments in insulin resistant conditions remain to be elucidated. In rodents, ANGPTL4 overexpression and treatment markedly decreased blood glucose concentration, improved glucose tolerance and hyperinsulinemia, but induced hyperlipidemia, fatty liver and hepatomegaly in mice [425]. The FA that are liberated after LPL-mediated lipolysis and those from the plasma FFA pool can be taken up in skeletal muscle via passive diffusion, depending on the concentration gradient over the muscle membrane and via membrane associated carrier proteins [426] like CD36, FABPpm and a family of fatty acid transport proteins (FATP 1-6) [427-430]. Of these FA transporters CD36 has been best characterized [46]. CD36 deficiency has been associated with a functionally important impairment in FA transport in muscle and adipose tissue [427, 431]. In humans, it has been shown that muscle CD36 protein expression may be acutely upregulated by insulin [80, 432] and that this upregulation may be more pronounced in insulin resistant conditions [80, 433]. Furthermore, it has been shown that CD36 translocation to the plasma membrane may be increased in muscle strips of obese subjects with T2D and that FA uptake, as measured with the giant vesicle model, was increased 4- 
fold as compared to overweight and lean controls [434]. Nevertheless, several human in vivo studies showed that the FFA concentrations were comparable between groups and no difference was observed in skeletal muscle FA uptake during fasting or insulin-stimulated conditions in obese versus lean subjects [434], in patients with impaired glucose metabolism versus normal glucose tolerant subjects [414, 435] or in insulin resistant men versus controls [436]. However, results are contradicting, since 2 other studies showed a small increase in muscle FFA uptake during fasting in obese versus lean [437] or insulin resistant versus control subjects [92], despite comparable FFA concentrations. In summary, there may be more human evidence for an elevation of plasma TAG concentrations as compared to FFA in the obese insulin resistant or pre-diabetic state. Although mixed evidence is available for increased muscle TAG extraction, not much evidence is available from in vivo human studies for the notion that skeletal muscle FFA uptake may be largely increased in the insulin resistant state.

\section{Skeletal muscle fatty acid storage}

The FAs that enter the myocyte, bind to cytoplasmic FABPc for transport through the cell [438] and can either be directed towards storage in lipid droplets or towards the mitochondria for oxidation. The synthesis of TAG in the myocyte involves the activity of several glycerol-3-phosphates (GPAT1-4), lipin 1 and DGAT1-2 [439] whilst the activity of SCD1 or $\Delta$-9-desaturase is particularly important for the further metabolism of SFA. Increased IMTAG synthesis via upregulation of lipogenic enzymes (DGAT1, GPAT an SCD1) has been linked to protection against FAinduced insulin resistance in rodents and humans [416]. Recently, Timmers et al. [440] showed that unilateral overexpression of DGAT-1 (involved in the conversion of TAG to DAG) in rat skeletal muscle, could rescue insulin sensitivity despite increased DAG and TAG concentrations, possibly by increasing DAG and TAG turnover [440]. Nevertheless, data on DGAT1 expression in human skeletal muscle are mixed with several studies showing no differences in DGAT1 expression between obese insulin resistant subjects and normal weight sedentary volunteers, endurance trained athletes [441] or in obese volunteers after weight loss [442]. One of the putative mechanisms regulating turnover of in particular SFAs may be related to the activity of SCD1 or $\Delta$-9-desaturase, converting SFAs to MUFAs. Overexpression of SCD1 has been reported to protect L6 myotubes from FAinduced insulin resistance and increased TAG reesterification [443]. Nevertheless, evidence is mixed with another study showing increased SCD1 expression in skeletal muscle of extremely obese humans with severe muscle insulin resistance [444]. Less information is available on the synthesis rate of muscle TAG in vivo in humans. Bergman and coworkers [445] showed that IMTAG concentration and its fractional synthetic rate were not related to insulin action in smokers compared to non-smokers [445]. Additionally, the same group showed that obese prediabetic men had higher muscle TAG concentrations, a lower TAG fractional synthetic rate and a lower oxidative capacity in parallel to a reduced insulin action as compared to obese normal glucose tolerant men [446]. These disturbances in IMTAG metabolism were not found in women. In line, we recently showed that a more pronounced degree of insulin resistance in subjects with IGT (either isolated or in 
combination with IFG) as compared to isolated IFG was accompanied by a reduced fractional synthesis of TAG from dietary palmitate, an increased saturation of the intramuscular FFA pool, a reduced saturation of the DAG and TAG pool and a reduced expression of genes involved in oxidative metabolism, confirming that a reduced muscle lipid synthesis and turnover may be an important characteristic of the insulin resistant muscle [447]. Finally, a reduced incorporation of FFA into TAG in primary myotubes from obese individuals with T2D has been recently observed. The data indicate that the ability to incorporate FAs into TAG is an intrinsic feature of human muscle cells that is reduced in individuals with T2D [448]. In summary, there is evidence that partitioning of FFA towards TAG synthesis may be beneficial for insulin sensitivity and that insulin resistance may be associated with a reduced fractional TAG synthesis in skeletal muscle. 


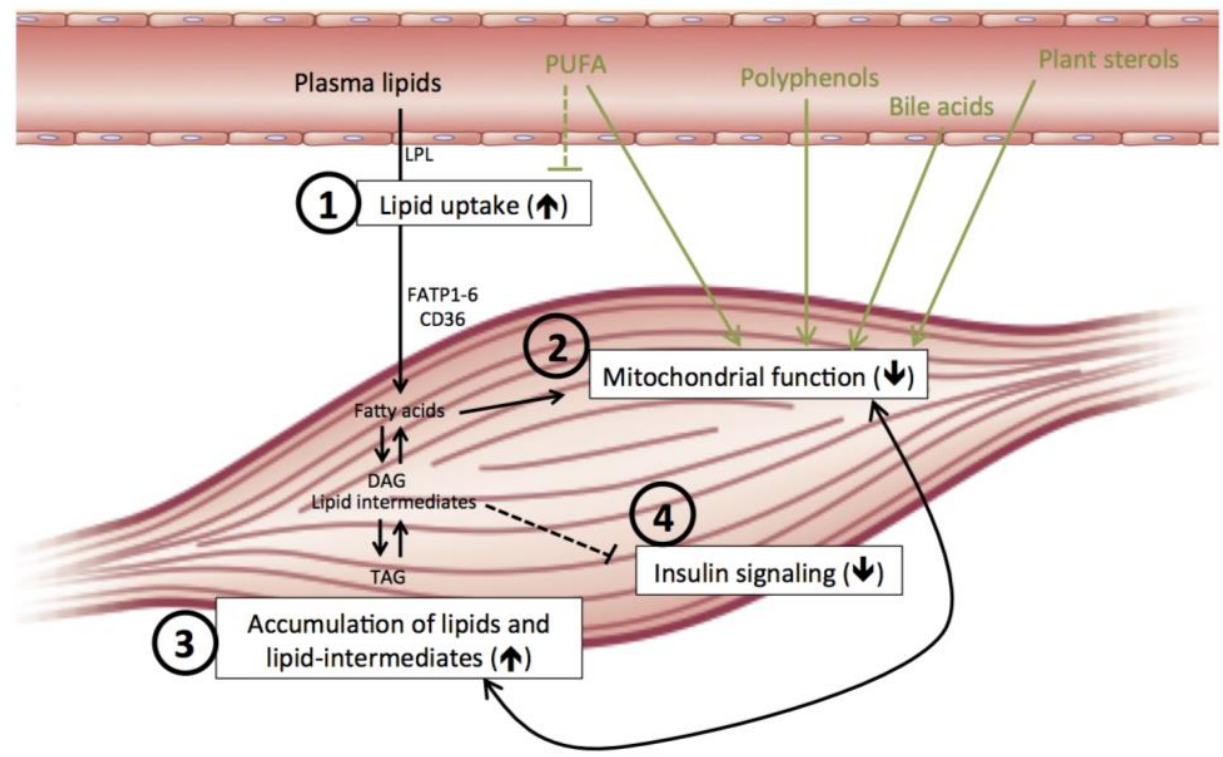

Figure 4. Disturbances in skeletal muscle fatty acid metabolism

Skeletal muscle fatty acid uptake, which is regulated via LPL and fatty acid transport proteins (FATP1-6, CD36), might be higher in subjects with impaired glucose metabolism compared to control subjects (1). In addition, a reduced mitochondrial mass and/or mitochondrial function (2) have been proposed as underlying mechanisms for reduced muscle fat oxidation, contributing to the accumulation of TAG and lipid-intermediates (LCFA-CoA, DAG, ceramides, acylcarnitines) in myocytes (3), interfering with insulin signaling (4) in the obese insulin resistant state. Finally, the capacity to increase intramyocellular fat oxidation during conditions of high FA supply has shown to be impaired in obese, T2D subjects. PUFA's, polyphenols, plant sterols and bile acids may have beneficial effects on skeletal muscle lipid metabolism.

Abbreviations: LPL: Lipoprotein lipase; FATP1-6: Fatty acid transport protein 1-6; CD36: Fatty acid translocase CD36; TAG: Triacylglycerol; DAG: Diacylglycerol; LCFA-CoA: long chain fatty acyl coenzyme A; PUFA: Poly unsaturated fatty acids. Dashed lines indicate inhibition. Solid lines indicate stimulation. Green lines indicate beneficial effects. Symbols: $(\boldsymbol{\uparrow})$ : Increased; $(\boldsymbol{\Downarrow})$ : Decreased 


\section{Skeletal muscle lipolysis}

Besides FA uptake and incorporation into DAG and TAG, also intrinsic disturbances in skeletal muscle lipolysis may contribute to the accumulation of lipids and lipid metabolites in skeletal muscle of obese insulin resistant subjects. As in adipose tissue, the intramuscular lipid stores are hydrolyzed by phosphorylation and translocation of ATGL and HSL, which are under hormonal control of catecholamines, NPs and insulin. HSL deficiency in mice has been shown to increase DAG storage and signs of impaired skeletal muscle insulin sensitivity [274], while ATGL deficient mice have increased TAG accumulation in skeletal muscle and show improved glucose tolerance and insulin sensitivity [449]. In line, in vitro data of Badin et al. [450] showed that overexpression of ATGL in human myotubes promotes DAG and ceramide accumulation and disrupts insulin signaling and action. These data suggest that a dysbalance in lipase expression and activity contributes to an increased accumulation of lipotoxic metabolites, which might interfere with insulin signaling. As far as we know, there is no human in vivo evidence for a significant improvement in insulin sensitivity following inhibition of muscle ATGL activity. However, it has been shown that ATGL deficiency in humans with neutral lipid storage disease with myopathy (NLSDM), when compared to healthy controls, show impaired insulin response to glucose, preserved whole-body insulin sensitivity and a shift toward glucose metabolism in the heart [451].

Furthermore, we showed that skeletal muscle of obese subjects exhibited a blunted $\beta$-adrenergic mediated lipolysis as compared to lean controls in vivo [452]. Additionally, under fasting conditions total glycerol release was reduced across forearm muscle of obese compared to lean men, which was accompanied by a reduced HSL protein content and an increased ATGL protein content without changes in CGI-58, a co-activator of ATGL [453]. This difference in lipase content was accompanied by a $60 \%$ lower ratio of DAG to TAG hydrolase activity implying incomplete muscle lipolysis. Nevertheless, the incomplete lipolysis was not accompanied by DAG accumulation, but total DAG content was rather decreased in obese subjects, again, as already indicated above not supporting an important role of total DAG content in lipid-induced insulin resistance. Furthermore, we recently showed that insulin-mediated suppression of skeletal muscle lipolysis is blunted in T2D compared to normal glucose tolerant subjects and that this is associated with increased accumulation of membrane saturated DAG and protein kinase C (PKC) activation [404]. These data are supported by several other observations that both cellular localization and composition of DAG influence the relationship to insulin sensitivity $[405,406]$. It remains to be elucidated whether intracellular lipases directly contribute to membrane DAG accumulation in human skeletal muscle. However, there are indications that in vitro ATGL mainly generates specific DAG isoforms (1,3-DAGs and 2,3-DAGs) but not 1,2-DAGs, of which the latter is found in the plasma membrane and able to activate PKC, suggesting that muscle lipases are not directly involved in membrane DAG accumulation [90]. Nevertheless, targeting skeletal muscle lipases may constitute an interesting strategy to improve insulin sensitivity in obesity and T2D.

Finally, accumulating evidence suggests that LD covering proteins play an important role in skeletal muscle lipid turnover and the development of insulin 
resistance. The best-characterized group of LD coat proteins is the PLIN family. As mentioned above, PLIN-1 is adipose tissue specific, although PLIN1 mRNA expression is observed in human skeletal muscle [454], while PLIN-2 and PLIN-5 mRNA and protein are abundantly expressed in skeletal muscle [455, 456]. PLIN-3 and PLIN-4 are also present in skeletal muscle, but little is known to date about their function in muscle lipid metabolism. It was recently reported that PLIN-5 protein is highly expressed in skeletal muscle of endurance athletes, corresponding to their higher LD volume and their higher insulin sensitivity [456]. Nevertheless, another study in humans showed no correlation between PLIN-5 protein content in skeletal muscle and insulin sensitivity in obese T2D subjects and BMI-matched control subjects [457]. PLIN-5 and PLIN-2 are co-localized with the LD [458] and recent research using in vitro and in vivo methods showed that PLIN-2 is an important facilitator of IMCL storage and that by improving IMCL storage, PLIN-2 protects against lipotoxicity improving thereby insulin sensitivity [455]. The role of LD dynamics in myocellular insulin resistance is beyond the scope of this review and has been reviewed in more detail elsewhere [399]. Taken together, although the field is still in its infancy, mounting evidence suggests a role for LD covering proteins in the protection against muscle lipotoxicity and insulin resistance. One could hypothesize that storage of lipids in LD is not as harmful as opposed to accumulating lipids elsewhere in the cell (e.g. the plasma membrane).

\section{Skeletal muscle fat oxidation}

The dynamics of fat oxidation and fine tuning with FA uptake and intramyocellular TAG turnover may be very important to prevent accumulation of bioactive lipid metabolites. To meet the complexity of changes in fuel oxidation, the concept of metabolic flexibility has been introduced, defined as the capacity to increase fat oxidation upon increased FA availability and to switch between fat and glucose as the primary fuel source after a meal [459]. The insulin resistant muscle may be characterized by a metabolic inflexibility to regulate substrate oxidation. Indeed, the capacity to increase intramyocellular fat oxidation during conditions of increased FA supply such as fasting has shown to be impaired in obese, T2D subjects [396, 460-462] and also during exercise and $\beta$-adrenergic stimulation in obese and T2D subjects [463, 464]. Additionally, the postprandial suppression of muscle fat oxidation has shown to be impaired in T2D and obesity [396, 435]. Together these data suggest that an impaired metabolic flexibility may contribute to the accumulation of lipid metabolites and may be driven by lipid supply from either extracellular or intracellular sources (lipid turnover) as well as intrinsic impairments in mitochondrial function.

Interestingly, a study in human myotubes indicated that elevated extracellular FAs increase their own oxidation, which may in turn inhibit the oxidation of intramyocellular lipids [465]. Also, inhibition of intracellular adipose tissue lipolysis with acipimox decreased adipose tissue derived FA availability and increased the oxidation of IMTAG derived FA during rest and during exercise [466]. Nevertheless, since circulating FFA concentrations may only be slightly elevated or not increased at all in insulin resistant conditions (see above), an increased lipid overflow from FFA is probably not a major contributor to an impaired utilization of IMTAG in the 
obese insulin resistance state. Since there is increasing support for the notion that lipid overflow in insulin resistance under physiological conditions is rather characterized by increased circulating TAG concentrations than by increased FFA concentrations, it is a plausible option that increased TAG-derived FA oxidation may impair IMTAG utilization in the obese insulin resistant state. However, this option remains to be investigated.

Furthermore, differences in IMTAG oxidation may be caused by differences in IMTAG lipolysis. Interestingly, lipolysis from IMTAG might be necessary for the maintenance of oxidative gene expression and FA oxidation. Recent findings indicate that in particular ATGL-mediated lipolysis generates lipid ligands for PPAR activation and the subsequent transcription of oxidative genes [90]. It was shown that a cycle of FA esterification and rehydrolysis is required for activation of PPAR$\alpha$ and normal mitochondrial and oxidative phosphorylation, at leasat in cardiomyocytes [467]. In vivo tracer data show that in patients with NLSDM (caused by mutations in the ATGL gene) fat oxidative capacity is blunted and can be partly reversed by PPAR- $\alpha$ treatment $[468,469]$. These data highlight the importance of ATGL activity in regulating muscle fat oxidation. Furthermore, it was shown recently that activation of NP signaling in human skeletal muscle enhances mitochondrial oxidative metabolism and fat oxidation [470]. However, further studies are required linking lipolysis and NP signaling to muscle oxidative metabolism and insulin resistance in human.

Finally, a reduced FA transport across the mitochondrial membrane and a reduced mitochondrial mass and/or mitochondrial function have been proposed as underlying causes of a reduced muscle fat oxidation in insulin resistance. In rodent models of obesity and insulin resistance an increased content of malonyl-CoA, an allosteric inhibitor of mitochondrial FA transport, has been shown in skeletal muscle in combination with hyperglycaemia and hyperinsulinaemia and a reduced lipid oxidation [471]. Also, in human skeletal muscle it has been shown that a combination of hyperglycaemia and hyperinsulinaemia increases malonyl-CoA, inhibits functional carnitine palmitoyl transferase 1 (CPT1b, muscle isoform) activity and shunts long chain FA away from oxidation and towards storage in human muscle [472]. Furthermore, two studies have shown that an improvement in fat oxidation by exercise or by lifestyle intervention was accompanied by decreased acetyl-CoA carboxylase (ACC) mRNA expression, involved in the formation of malonyl-CoA, suggesting that a reduced inhibition of mitochondrial FA transport through malonyl-CoA may have contributed to the improved fat oxidative capacity [473, 474]. Furthermore, a lowered mitochondrial transport through CPT1 may contribute to the reduced fat oxidation. In line, heterozygous CPT1b deficiency results in mitochondrial abnormalities and lipid accumulation with elevated TAG and ceramide content at least in mice cardiomyocytes [475]. Moreover, it has been shown that an increased CPT1b activity is sufficient to improve high-fat diet induced insulin resistance [476]. Peroxisome proliferator-activated receptor gamma coactivator-1alpha (PGC-1 $\alpha$ ) is involved in mitochondrial biogenesis by supporting the transcriptional activity of nuclear respiration factor 1 (NRF1), thereby regulating the transcription of genes involved in oxidative metabolism. The expression of genes responsible for oxidative phosphorylation is coordinately downregulated in muscle of T2D individuals $[477,478]$ and muscle specific overexpression of PGC$1 \alpha$ in rodents has been shown to improve lipid utilization, insulin signaling and 
glucose transport [479]. In vivo mitochondrial capacity and function have been reported to be reduced in T2D, independent of mitochondrial content [388, 480, 481], although data are not consistent [482, 483]. Nevertheless, a reduced mitochondrial capacity and a reduced activity of TCA cycle [484] and citrate synthase $(-14 \%)$ [485] have also been reported in primary myotubes from T2D subjects, suggesting that skeletal muscle mitochondrial function is intrinsically impaired in insulin resistance. Moreover, a marked reduction in mitochondrial oxidative and phosphorylation activities, together with intramyocellular lipid accumulation, were found in skeletal muscle in insulin resistant offspring of T2D subjects [486, 487]. Furthermore, the definition of mitochondrial dysfunction may also include a dysbalance balance between $\beta$-oxidation and TCA cycle activity resulting in an accumulation of incompletely oxidized, fatty acid products (i.e. acylcarnitines), which can impair skeletal muscle insulin sensitivity, as extensively reviewed elsewhere [488].

In summary, skeletal muscle fat oxidation is reduced in the obese insulin resistant state. This impaired fat oxidation may contribute to the accumulation of lipid metabolites and may be driven by lipid supply from either extracellular or intracellular sources as well as intrinsic impairments in mitochondrial function. However, whether reduced mitochondrial function is causally related to insulin resistance or rather a consequence of the sedentary lifestyle needs to be investigated in more detail in future research.

\section{Putative nutrional targets to improve skeletal muscle lipid metabolism}

An improvement in adipose tissue and liver function may reduce lipid overflow and low-grade systemic inflammation, thereby reducing the supply of lipids and inflammatory mediators to skeletal muscle, which likely improves skeletal muscle function.

As indicated above, targeting skeletal muscle lipases, LD covering proteins and skeletal muscle fat oxidation/mitochondrial function to improve metabolic flexibility may constitute an interesting strategy to improve insulin sensitivity in obesity and T2D by limiting the accumulation of bioactive lipid metabolites interfering with insulin sensitivity.

As discussed earlier in this review, specific polyphenols or a combination of polyphenols may affect human lipid metabolism. The polyphenolic compound resveratrol, which is abundantly present in grape skin, red wine and peanuts, has been shown to improve in vitro FA oxidation and insulin sensitivity in murine adipocytes [489], as well as in C2C12 myotubes [490] and improved skeletal muscle mitochondrial function in rodents by activating Sirtuin-1 (SIRT-1) and PGC1a [491]. In humans, on the other hand, resveratrol supplementation has recently been shown to improve metabolic profile and flexibility, skeletal muscle mitochondrial function, energy expenditure, glucose control and insulin sensitivity especially in patients with T2D $[492,493]$. We have recently also shown that shortterm supplementation of the combination of EGCG and resveratrol may increase resting energy expenditure and improve metabolic flexibility [212]. Nevertheless, results of clinical trials are contradictory, as recently discussed [494].

Additionally, dietary fat quality may differentially affect (skeletal muscle) lipid 
metabolism and insulin sensitivity [218, 495] Indeed, we recently showed that a PUFA rich meal acutely improved insulin sensitivity in obese insulin resistant subjects as compared to a high-SFA meal, which was accompanied by a reduced TAG-derived skeletal muscle FA uptake, an altered intramyocellular lipid partitioning and a more oxidative transcriptional phenotype [496]. Additionally, studies in human myotubes showed that palmitate induced a lower lipolysis rate [497] and follows a different metabolic pathway as compared to oleic acid [497,498]. Also, a lower palmitate oxidation has been shown in myotubes of T2D subjects as compared to control, whilst oleate oxidation was not significantly different [499]. These studies indicate that dietary fat quality may modulate the nature of lipid-induced insulin resistance through affecting pathways of fatty acid partitioning. Further studies are required to investigate the impact of gender and dietary fat quality on FA dynamics and to investigate the causative role of an impaired TAG synthesis capacity in the etiology of insulin resistance. In summary, polyphenols and modulation of dietary fat quality may affect storage of bioactive lipid metabolites in skeletal muscle, thereby improving insulin sensitivity and glucose homeostasis. Beside that, manipulation the nuclear FXR and LXR receptors by modulating bile acid metabolism or by ingestion of plant sterols may also have positive effects on muscle lipid metabolism and insulin sensitivity, but apparently more human evidence is required. For a summary see Table 1. 


\begin{tabular}{|c|c|c|c|c|c|c|c|c|c|c|c|c|c|c|c|}
\hline 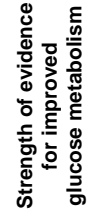 & 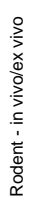 & 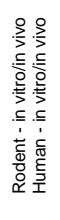 & 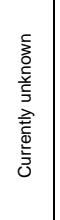 & 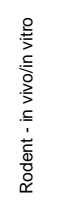 & 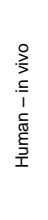 & 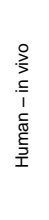 & 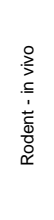 & 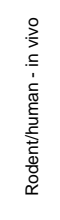 & 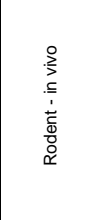 & 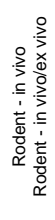 & 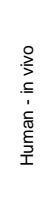 & 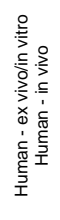 & 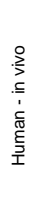 & 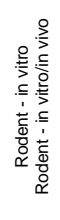 & 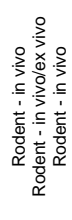 \\
\hline 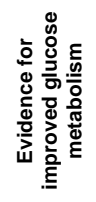 & 䔅 & $\begin{array}{l}\bar{\Xi} \\
\overline{0} \\
\stackrel{0}{\underline{0}}\end{array}$ & 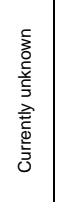 & 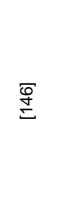 & 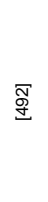 & $\stackrel{E}{E}$ & $\begin{array}{l}\frac{\sigma}{\sigma} \\
\underline{\underline{\theta}}\end{array}$ & $\stackrel{\text { M. }}{\underline{I}}$ & 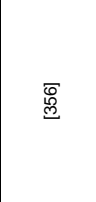 & 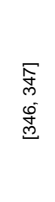 & 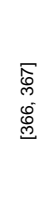 & 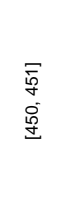 & $\begin{array}{l}\text { 厓 } \\
\end{array}$ & 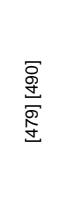 & 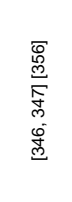 \\
\hline 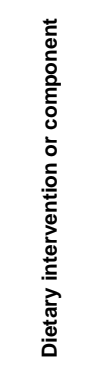 & 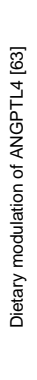 & 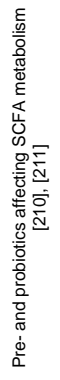 & 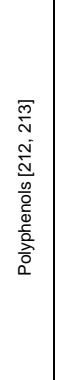 & 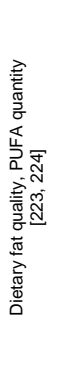 & 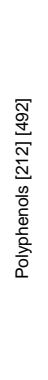 & 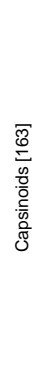 & 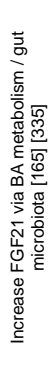 & 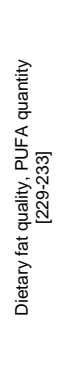 & 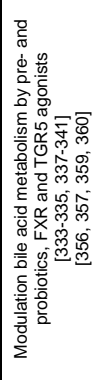 & 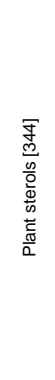 & 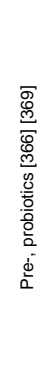 & 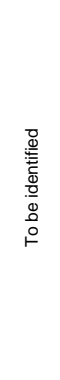 & 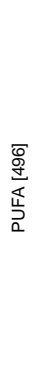 & 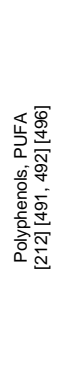 & 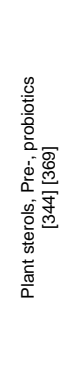 \\
\hline 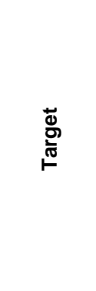 & 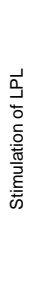 & 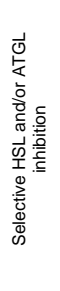 & 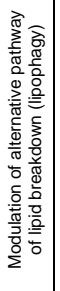 & 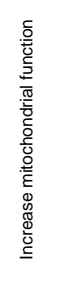 & & 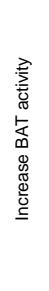 & & 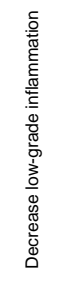 & 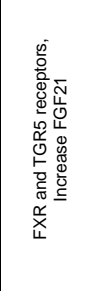 & 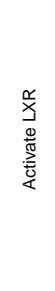 & 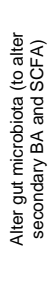 & 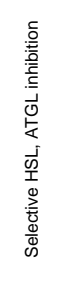 & 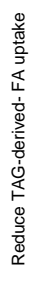 & 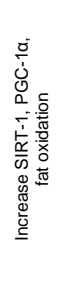 & 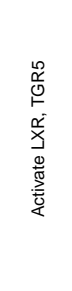 \\
\hline 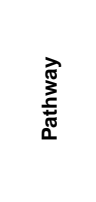 & 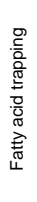 & 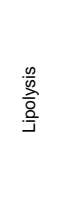 & $\begin{array}{l}\frac{.0}{0} \\
\frac{0}{0} \\
\frac{0}{3}\end{array}$ & 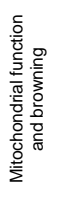 & & & & 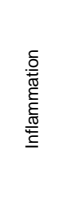 & 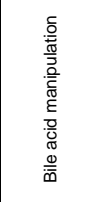 & 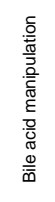 & 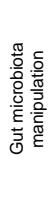 & $\begin{array}{l}\frac{0}{\underline{n}} \\
\frac{\bar{\partial}}{3}\end{array}$ & 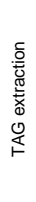 & 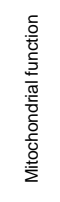 & 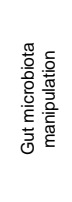 \\
\hline 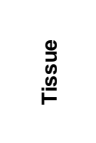 & 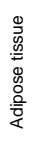 & & & & & & & & 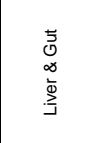 & & & 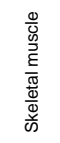 & & & \\
\hline
\end{tabular}




\section{PANCREATIC BETA-CELL FUNCTION}

T2D is a multifactorial disease that develops when insulin resistance is accompanied by pancreatic $\beta$-cell failure [2, 3], which is defined as a deterioration of $\beta$-cell function as well as loss of functional $\beta$-cell mass. In obese conditions, lipid accumulation of TAG in pancreatic islets occurs [500], which is aggravated by the simultaneous presence of hyperglycemia [501-503]. Elevated lipid accumulation can damage the $\beta$-cell functionality (Figure 5 ) and evidence for this mainly comes from studies with isolated islets exposed to high concentrations of FFA for periods of 24-48 $\mathrm{h}$ [504, 505], while evidence from in vivo studies mainly comes from studies with ZDF rats, since suitable pancreas specimens from individuals with increasing degrees of $\beta$-cell dysfunction are scarce [235]. This decreased $\beta$-cell functionality may lead to a decreased insulin secretion even when demands for secretion are increased. However, in order to maintain normal glucose homeostasis, a compensatory increased insulin secretion occurs. Prediabetic patients however, in the long run, fail to compensate adequately for the greater insulin requirements $[2,506-508]$ and will ultimately develop T2D. In this type 2 diabetic state, a decreased $\beta$-cell mass [509] and an increased level of $\beta$-cell apoptosis [510-514] occurs. However, the rate of $\beta$-cell replication and neogenesis was not different between a diabetic and a control group [510].

\section{Putative targets to improve insulin secretion}

The increased rate of $\beta$-cell apoptosis in T2D can originate from several mechanisms [515], including oxidative stress [13, 509, 516, 517], inflammation [518, 519], ER-stress [520-523], mitochondrial overload [524, 525] and lipotoxicity [526]. As extensively reviewed elsewhere, human and animal data suggest that there might be direct effects of bioactive compounds (including polyphenols, vitamins and carotenoids) on enhancing insulin secretion and preventing $\beta$-cell apoptosis, and some compounds might modulate $\beta$-cell proliferation [527]. Targeting these processes within the pancreatic islets, as already discussed in the above paragraphs, may result in decreased $\beta$-cell apoptosis and maintain the insulin secretory capacity, and as such could be a potential therapy to reduce the incidence of T2D. 


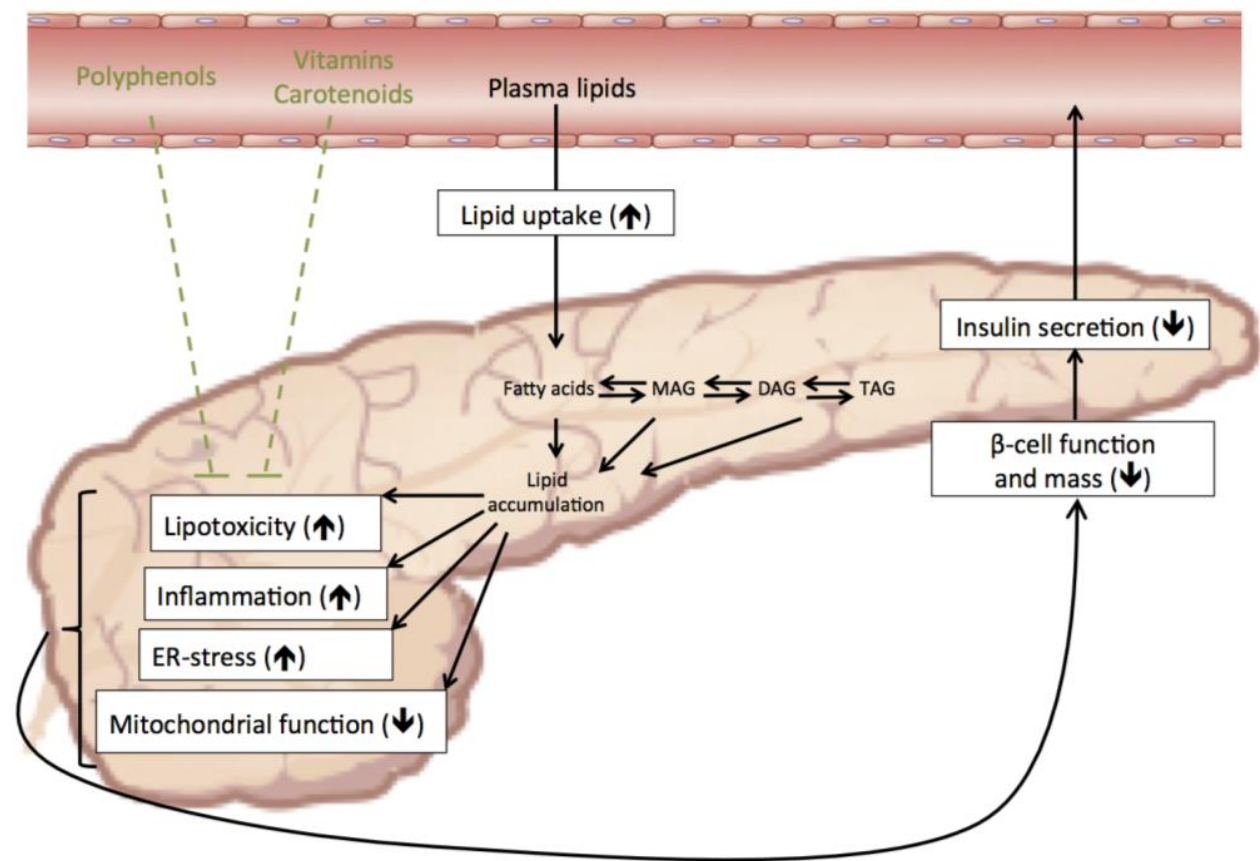

Figure 5. Disturbances in pancreatic fatty acid metabolism

Lipid accumulation in pancreatic islets can result in inflammation, ER-stress, mitochondrial overload and lipotoxicity. These mechanisms damage the $\beta$-cell function and lower $\beta$-cell mass, resulting in a decreased insulin secretion and ultimately leading to the development of T2D. Polyphenols, vitamins and carotenoids may counteract these negative effects.

Abbreviations: TAG: Triacylglycerol; DAG: Diacylglycerol; MAG: monoacylglycerol; FFA: Free fatty acids; ER-stress: Endoplasmic reticulum stress. Dashed lines indicate inhibition. Solid lines indicate stimulation. Green lines indicate beneficial effects. Symbols: $(\boldsymbol{\uparrow})$ : Increased; $\boldsymbol{\downarrow})$ : Decreased 


\section{THE INTESTINE AND GUT MICROBIOTA IN INSULIN RESISTANCE}

The intestinal tract has traditionally been seen as a passive organ, but currently it is seen as an active organ that may affect substrate metabolism via the secretion of several factors such as free fatty acids, incretins and inflammatory factors [528] and may contribute to the development of obesity and insulin resistance $[427,529$, 530]. The intestine plays an important role in lipid homeostasis and contributes significantly to the systemic plasma cholesterol and lipid concentrations [531]. Fat absorption by the small intestine is a very efficient process, which requires several transport mechanisms, as reviewed elsewhere [532]. Fatty acids taken up in the enterocyte can either be oxidized by the enterocyte, [533-535], stored as intracellular LD's [536] or excreted as chylomicrons, which is related to hypertriglyceridemia [537, 538] contributing to T2D [539], metabolic syndrome [540] and coronary artery disease [541, 542]. Targeting these processes can significantly affect postprandial lipid concentrations and insulin sensitivity. Improving intestinal fatty acid oxidation, for example by PPAR- $\alpha$ activation [543] might contribute to reduced lipid accumulation in other organs, such as liver and skeletal muscle. Furthermore, also PUFAs [544, 545] and DAG [546] appear to be effective inducers of enterocyte fatty acid oxidation and may be beneficial in the anti-diabetic and anti-obesity effects in various tissues. Besides fatty acid oxidation, also the absorption and excretion of fatty acids might be altered. As reviewed elsewhere, there are several components that might influence intestinal lipid metabolism [547]. For example, JTT-130, a novel intestine-specific inhibitor of microsomal triglyceride transfer protein (MTP), which is involved in the mobilization and secretion of TAG rich lipoproteins from enterocytes and hepatocytes [548], has been shown to suppress the absorption of dietary fat and cholesterol in the intestine, decreased plasma TAG and total cholesterol levels [549] and improved glucose and lipid metabolism in rodents [550-552].

Furthermore, recent evidence showed that a high-fat diet induced intestinal inflammation [553, 554], which is related to obesity and insulin resistance [555557]. Interestingly, it has recently been shown in beta7 integrin-deficient mice (Beta7(null)) that suppression of the gut immune system decreases HFD-induced insulin resistance [558]. Furthermore, intestinal ER stress has been found in obesity [559] and might contribute to intestinal and systemic inflammation [560], leading to insulin resistance [561]. Taken together, this makes the intestine an attractive therapeutic target.

Importantly, not only the intestine itself, but also its microbiota play an important role in the intestinal lipid metabolism, obesity and insulin resistance. The human adult intestines contain more than $10^{14}$ bacteria from over 1000 species and the genetic material of the intestinal microbes is collectively called the microbiome [562]. The vast majority of all gut microbes include the phyla Gram-negative Bacteroidetes, Proteobacteria and Verrucomicrobia as well as the Gram-positive Firmicutes and Actinobacteria [562-566]. Growing evidence indicates that also our gut and its microbiota play a crucial role in substrate metabolism and the development of obesity, cardiometabolic diseases and T2D, as reviewed extensively elsewhere [567, 568] (Figure 6).

Obese and T2D subjects seem to be characterized by an altered composition of gut microbiota compared to lean and normal glucose tolerant individuals, 
respectively [569-572], although conflicting results have also been reported [570, 573-575]. Interestingly, germ-free mice infected with the gut microbiota of conventionally raised mice demonstrated increased body weight, insulin resistance and glucose intolerance together with reduced food intake and increased oxygen consumption [576]. The body fat gain was related to suppressed gut expression of the LPL inhibitor ANGPTL4, leading to increased fat accumulation in adipocytes and increased energy harvest [570, 577-579].

In obese men, seven days treatment with the antibiotic vancomycin modulated their gut microbiota and decreased peripheral insulin sensitivity [364]. Finally, the transfer of intestinal microbiota from human lean donors to individuals with the metabolic syndrome increased insulin sensitivity, along with increase of intestinal butyrate-producing microbiota [580].

Several mechanisms link our gut microbiota to obesity and diabetes (diabesity), as extensively reviewed elsewhere [581]. Firstly, alterations in gut microbiota composition can increase the amount of LPS through increased production, reduced breakdown or increased translocation across the gut wall, thereby inducing a strong immune response to protect the organism from bacterial infection [582]. This LPS-induced inflammatory state is referred to as 'metabolic endotoxemia' and is accompanied by body weight gain and insulin resistance in animal models [583]. In addition, major microbial products from the fermentation of indigestible carbohydrates, like the SCFAs acetate, butyrate and propionate may affect energy and fat metabolism through various mechanisms [584-587]. The type of substrate is the primary determinant of the SCFA production rate and SCFA ratio, compared to the composition of the intestinal microbiota [584] and the level of substrate fermentability varies with fiber solubility. Soluble fibers are fermented more completely than insoluble fibers [588] and highly fermentable substrates produce higher amounts of SCFA [589], which have physiological effects in different tissues [590]. At first, it has been proposed that they may play a role in harvesting extra energy from the diet, which would be a promoting factor for body weight gain. However, evidence that this largely contributes to human energy balance is not yet convincing [591]. On the other hand, increased SCFA concentrations have rather been associated with distinct generally positive, metabolic effects, affecting hormonal release of glucagon-like peptide 1 (GLP1) and peptide YY (PYY), cell proliferation and differentiation [584, 592-595], regulate adipocyte lipolysis [210, 211], modulate inflammation, affecting adipose tissue fat storage and ectopic fat storage, as has been extensively reviewed elsewhere [596]. Effects of SCFAs may be related to the activation of their related G-protein coupled receptors GPR41 (also known as free fatty acid receptor 3 (FFAR3)) and GPR43 (FFAR2) [596], which are present in the gut epithelium but also in peripheral tissues like adipose tissue and skeletal muscle [595, 597]. Thus, manipulation of SCFA production by prebiotics and probiotics (or a combination of both) may, among other processes, differentially affect human fat and glucose metabolism.

Furthermore, besides converting non-digestible fibers, the microbiota are also closely linked to BA metabolism. On the one hand, microbiota can transform primary to the so-called secondary BAs [598], while on the other hand BAs by themselves exert an anti-microbial effect on the microbiota [599, 600]. As discussed above, the secondary BAs can affect host metabolism via binding to 
several nuclear receptors and GPRs and altering the intestinal microbiota could be a potential therapeutic target to reduce obesity and insulin resistance.

\section{Putative nutritional targets to modulate gut microbiota composition and function}

Altering the diet, either via specific dietary FA or combinations of pre- and probiotics, can rapidly change the human gut microbiota [601-603] and can improve metabolic status [604, 605]. By fermenting dietary fibers that humans cannot (fully) digest themselves, the human microflora produces SCFA [565, 570, $588,606]$, which may play an important role in glucose homeostasis via modulation of human substrate metabolism, as briefly discussed above. Besides endogenous SCFA production, exogenous administration of SCFA has been studied in the context of interest. Thus, an alteration of the SCFA production by microbiota, i.e. via intake of pre- and probiotics, may be a useful tool to prevent obesity and obesity-related insulin resistance. Additionally, prebiotics may also directly affect postprandial glycemic and insulinemic responses by slower glucose absorption [607].

Research has shown that FAs exert their effect on bacterial growth and survival, in part, via altering the microbial cell membrane fluidity and disrupting cell membranes of certain bacteria [608, 609]. As reviewed by Alcock et al. [610], unsaturated fatty acids had a higher anti-microbial effect compared to SFA and within the group of SFA, short chain saturated FA (4 to 12 carbons in length) had a higher bactericidal effect, compared to long chain saturated FA (more than 12 carbons). Furthermore, while MUFA had a lower anti-microbial effect then PUFA of the same chain length, the n-3 PUFA had a greater effect compared to n-6 PUFA, but results are not consistent [610]. Although human evidence is scarce, research showed that the type of dietary fat has an effect on the faecal microbiota. Fava et al. [611] showed that a diet high in SFA increased Faecalibacterium prausnitzii and that MUFA-rich diets reduced the total bacterial numbers, but not the specific bacterial groups. In contrast, Simões et al. [612] showed that high habitual intake of MUFA was associated with lower numbers of bifidobacteria and slightly higher numbers of Bacteroides spp., that habitual n-3 PUFA intake had a significant positive association with Lactobacillus group abundance and that a higher habitual n-6 PUFA intake was associated with a decreased number of bifidobacteria. Santacruz et al. [613] showed that the numbers of Lactobacilli remained the same, eventhough the total PUFA consumption was greatly reduced.

Furthermore, it was shown that a high-fat diet significantly reduced the bifidobacteria [614]. In addition, high-fat diets seem to increase metabolic endotoxemia, which is related to an inflammatory state [583, 615, 616].

The altered gut microbiota composition also affects BA metabolism in rodents [617, 618] as well as in humans [364]. As such, it might be a possible target to combat the development towards obesity and insulin resistance (Table 1), as discussed above. However, further human studies are necessary to understand the exact mechanisms of action of the human microbiome. 


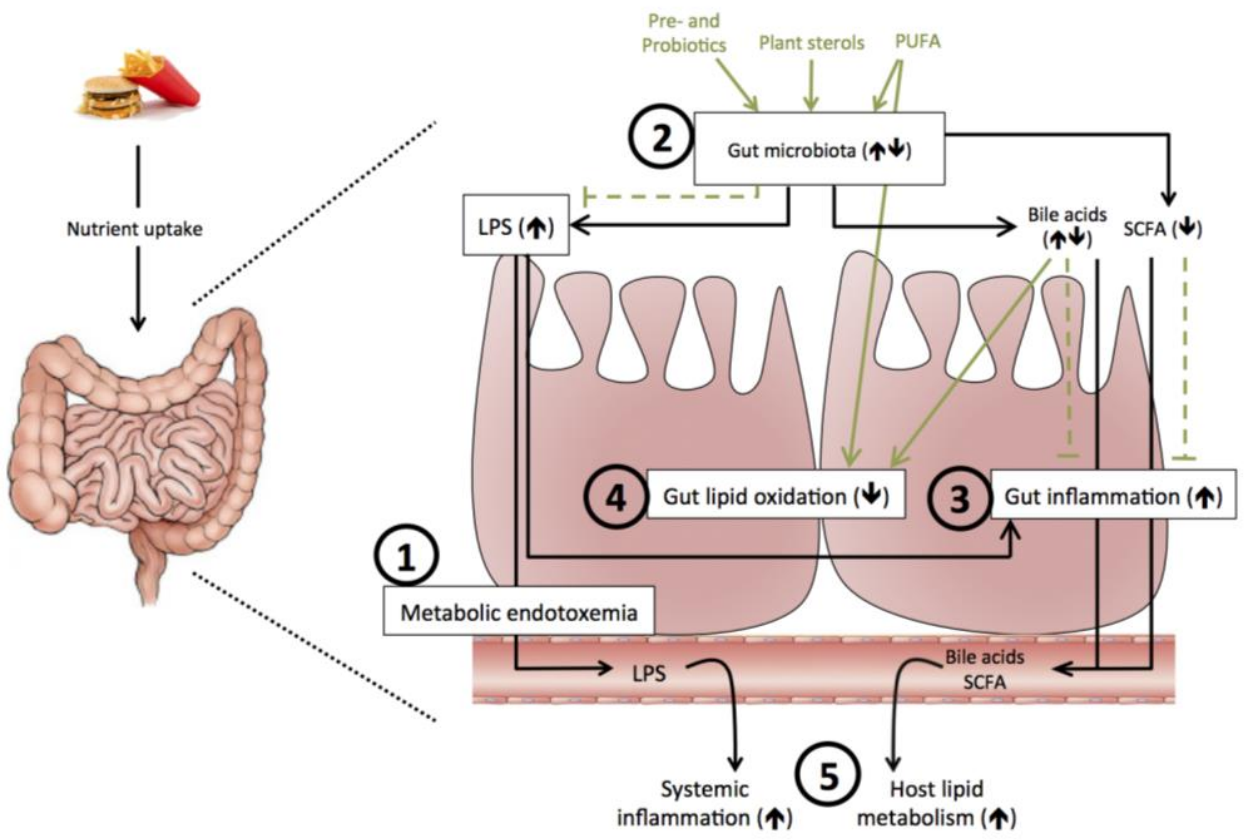

Figure 6. Disturbances in gut microbiota composition and lipid metabolism in the gut affect host metabolism

An altered gut microbiota composition, as seen in obesity and T2D, may increase local and systemic lipopolysaccharide (LPS) concentrations, referred to as metabolic endotoxemia (1) that can lead to systemic inflammation. Besides LPS, also bile acid composition and SCFA concentrations are altered. Pre- and probiotics, plant sterols and PUFAs exert a beneficial effect on gut microbiota composition (2), leading to an altered SCFA production, bile acid composition and a decreased LPS concentration, thereby affecting gut inflammation (3) and gut lipid metabolism (4). In addition, changes in systemic SCFA, bile acids and LPS affect host lipid metabolism and inflammation in adipose tissue, liver and skeletal muscle (5).

Abbreviations: PUFA: Poly unsaturated fatty acids; SCFA: short chain fatty acid; LPS: Lipopolysaccharide. Dashed lines indicate inhibition. Solid lines indicate stimulation. Green lines indicate beneficial effects. Symbols: $(\boldsymbol{} \downarrow)$ : Altered; $(\boldsymbol{\uparrow})$ : Increased; $(\boldsymbol{\downarrow})$ : Decreased 


\section{SUMMARY}

This review focuses on the disturbances in fatty acid metabolism at both the tissue and whole-body level that play a role in the etiology of insulin resistance, $\beta$-cell dysfunction and an impaired glucose metabolism. The most important pathways related to lipid metabolism in adipose tissue, liver, skeletal muscle, pancreas and gut have been discussed, and targets to improve lipid metabolism and glycaemia, which may be modulated by diet, have been described. A detailed overview of pathways and potential targets to improve lipid metabolism and glycaemia in adipose tissue, liver, skeletal muscle, pancreas and gut, which can be modulated by dietary interventions and food components, is provided in Table 1, and will be explained in more detail below.

Within adipose tissue, the balance between fatty acid extraction, lipolysis, adipocyte differentiation and mitochondrial function is important to maintain adequate lipid storage capacity. Improving the lipid storage capacity of adipose tissue prevents lipid overflow in the circulation and subsequent ectopic fat deposition, and therefore has high potential to improve glucose tolerance and insulin sensitivity. Increasing adipose tissue fatty acid uptake, possibly via stimulation of LPL, might reduce lipid overflow. On the other hand, a reduced lipid overflow might also be achieved by partial inhibition of lipolysis (partial lipase inhibition) and/or by modulation of the alternative pathway of lipid breakdown (lipophagy). There is some evidence that partial lipolysis inhibition may be achieved by modulation of SCFA metabolism through pro- and prebiotics and that the lipophagic pathway may be modulated by specific polyphenols, like resveratrol. Furthermore, adipose tissue mitochondrial function seems important in balancing lipid supply and utilization, and thereby affects adipose tissue function. Improved adipose tissue mitochondrial function may be achieved by nutritional strategies such as supplementation with specific polyphenols (or a combination of polyphenols) and altering dietary fatty acid composition. Dietary fat quality not only modulates lipid metabolism but may also affect low-grade inflammation, which in turn may lower the risk of developing insulin resistance and T2D.

An attractive target to tackle obesity and insulin resistance could be manipulation of the bile acid metabolism and/or gut microbiota composition. On the one hand, bile acids influence energy expenditure and glucose homeostasis via their effects on gluconeogenesis, insulin secretion and insulin sensitivity, and an altered concentrations of secondary bile acids can affect host metabolism via binding to several nuclear receptors (e.g. FXR, LXR, RXR), some of which can be activated by sitosterol, campesterol and certain oxidized derivatives of phytosterols (oxyphytosterols). On the other hand, an alteration of the gut microbiota by modulation of the dietary fat quality or pre- and probiotics might have a distinct effect on bile acid composition and metabolism and may also affect fermentation products from dietary fibers (e.g. SCFA and monosaccharides), affecting thereby liver gluconeogenesis and lipogenesis, pathways directly or indirectly affecting glucose homeostasis.

Targeting skeletal muscle lipid turnover and the balance between lipolysis, FA uptake and mitochondrial function/fat oxidation may constitute an interesting strategy to improve insulin sensitivity in obesity and T2D by limiting the accumulation of bioactive lipid-intermediates interfering with insulin sensitivity and 
glucose homeostasis. The most promising dietary intervention to reduce FA uptake and thereby muscle fat accumulation might be an improvement of the adipose tissue lipid buffering capacity through the manipulation of dietary fat quality. On the other hand, an increased activation of SIRT-1 and PGC-1a may result in an enhanced skeletal muscle mitochondrial biogenesis/function, fat oxidation and metabolic flexibility. Putative dietary strategies for the latter pathways may be modulation of the dietary polyphenol content or the modulation of dietary fat quality. In addition, stimulation of the nuclear FXR and LXR receptors by modulation of bile acid metabolism or by ingestion of plant sterols may have positive effects on skeletal muscle lipid metabolism and insulin sensitivity.

Finally, preventing $\beta$-cell apoptosis, and modulation of $\beta$-cell proliferation by bioactive compounds (including polyphenols, vitamins and carotenoids) may maintain the insulin secretory capacity, and as such could be a potential target to reduce the incidence of $\mathrm{T} 2 \mathrm{D}$.

To conclude, the main most promising dietary interventions or components to target fatty acid metabolism in order to improve glucose metabolism would be components to alter the gut microbiota composition, like pre- and probiotics, thereby affecting SCFA- and bile acid metabolism. Furthermore, the use of (specific) polyphenol(s) to increase adipose tissue function possibly by affecting the lipolytic pathway or the alternative pathway for lipid breakdown (lipophagy) and/or adipose tissue and skeletal muscle mitochondrial function may be promising strategies to improve glucose metabolism. Lastly, manipulation of the dietary fat quantity and quality is of particular interest to improve adipose tissue function and skeletal muscle lipid turnover and mitochondrial function. Further human intervention studies are required targeting specific fatty acid related pathways to translate the above indications into relevance for the control of glucose homeostasis and insulin sensitivity. 


\section{REFERENCES}

1. Reaven GM. Banting lecture 1988. Role of insulin resistance in human disease. Diabetes 1988; 37: 1595-1607.

2. Relimpio F. "The relative contributions of insulin resistance and beta-cell dysfunction to the pathophysiology of Type 2 diabetes", by Kahn SE. Diabetologia 2003; 46: 1707.

3. Bergman RN, Ader M, Huecking K, Van Citters G. Accurate assessment of beta-cell function: the hyperbolic correction. Diabetes 2002; 51 Suppl 1: S212-220.

4. Roumen C, Blaak EE, Corpeleijn E. Lifestyle intervention for prevention of diabetes: determinants of success for future implementation. Nutr Rev 2009; 67: 132-146.

5. Tuomilehto J. Nonpharmacologic therapy and exercise in the prevention of type 2 diabetes. Diabetes Care 2009; 32 Suppl 2: S189-193.

6. Mensink M, Blaak EE, Wagenmakers AJ, Saris WH. Lifestyle intervention and fatty acid metabolism in glucose-intolerant subjects. Obes Res 2005; 13: 1354-1362.

7. Goossens $\mathrm{GH}$. The role of adipose tissue dysfunction in the pathogenesis of obesityrelated insulin resistance. Physiol Behav 2008; 94: 206-218.

8. Unger $\mathrm{RH}$, Clark GO, Scherer PE, Orci L. Lipid homeostasis, lipotoxicity and the metabolic syndrome. Biochim Biophys Acta 2010; 1801: 209-214.

9. Virtue S, Vidal-Puig A. Adipose tissue expandability, lipotoxicity and the Metabolic Syndrome--an allostatic perspective. Biochim Biophys Acta 2010; 1801: 338-349.

10. Snel M, Jonker JT, Schoones J, et al. Ectopic fat and insulin resistance: pathophysiology and effect of diet and lifestyle interventions. Int J Endocrinol 2012; 2012: 983814.

11. DeFronzo RA, Jacot E, Jequier E, Maeder E, Wahren J, Felber JP. The effect of insulin on the disposal of intravenous glucose. Results from indirect calorimetry and hepatic and femoral venous catheterization. Diabetes 1981; 30: 1000-1007.

12. Perseghin G, Scifo P, De Cobelli F, et al. Intramyocellular triglyceride content is a determinant of in vivo insulin resistance in humans: a $1 \mathrm{H}-13 \mathrm{C}$ nuclear magnetic resonance spectroscopy assessment in offspring of type 2 diabetic parents. Diabetes 1999; 48: 1600-1606.

13. Schrauwen P, Hesselink MK. Oxidative capacity, lipotoxicity, and mitochondrial damage in type 2 diabetes. Diabetes 2004; 53: 1412-1417.

14. Phielix E, Mensink M. Type 2 diabetes mellitus and skeletal muscle metabolic function. Physiol Behav 2008; 94: 252-258.

15. Goodpaster BH, Theriault R, Watkins SC, Kelley DE. Intramuscular lipid content is increased in obesity and decreased by weight loss. Metabolism 2000; 49: 467-472.

16. Krssak M, Falk Petersen K, Dresner A, et al. Intramyocellular lipid concentrations are correlated with insulin sensitivity in humans: a $1 \mathrm{H}$ NMR spectroscopy study. Diabetologia 1999; 42: 113-116.

17. Pan DA, Lillioja S, Kriketos AD, et al. Skeletal muscle triglyceride levels are inversely related to insulin action. Diabetes 1997; 46: 983-988.

18. Byrne CD, Brindle NP, Wang TW, Hales CN. Interaction of non-esterified fatty acid and insulin in control of triacylglycerol secretion by Hep G2 cells. Biochem $J$ 1991; 280 ( Pt 1): 99-104.

19. Fanelli C, Calderone S, Epifano L, et al. Demonstration of a critical role for free fatty acids in mediating counterregulatory stimulation of gluconeogenesis and suppression of glucose utilization in humans. J Clin Invest 1993; 92: 1617-1622.

20. Ferrannini E, Barrett EJ, Bevilacqua S, DeFronzo RA. Effect of fatty acids on glucose production and utilization in man. J Clin Invest 1983; 72: 1737-1747.

21. Lewis GF, Vranic M, Harley P, Giacca A. Fatty acids mediate the acute extrahepatic effects of insulin on hepatic glucose production in humans. Diabetes 1997; 46: 11111119. 
22. Hennes MM, Dua A, Kissebah AH. Effects of free fatty acids and glucose on splanchnic insulin dynamics. Diabetes 1997; 46: 57-62.

23. Svedberg J, Bjorntorp P, Smith U, Lonnroth P. Free-fatty acid inhibition of insulin binding, degradation, and action in isolated rat hepatocytes. Diabetes 1990; 39: 570574.

24. Wiesenthal $\mathrm{SR}$, Sandhu $\mathrm{H}, \mathrm{McCall} \mathrm{RH}$, et al. Free fatty acids impair hepatic insulin extraction in vivo. Diabetes 1999; 48: 766-774.

25. Frayn KN, Williams $\mathrm{CM}$, Arner $\mathrm{P}$. Are increased plasma non-esterified fatty acid concentrations a risk marker for coronary heart disease and other chronic diseases? Clin Sci (Lond) 1996; 90: 243-253.

26. Tikellis C, Wookey PJ, Candido R, Andrikopoulos S, Thomas MC, Cooper ME. Improved islet morphology after blockade of the renin- angiotensin system in the ZDF rat. Diabetes 2004; 53: 989-997.

27. Cani PD, Delzenne NM. Interplay between obesity and associated metabolic disorders: new insights into the gut microbiota. Curr Opin Pharmacol 2009; 9: 737743.

28. Delzenne NM, Cani PD. Gut microbiota and the pathogenesis of insulin resistance. Curr Diab Rep 2011; 11: 154-159.

29. Delzenne NM, Neyrinck AM, Cani PD. Modulation of the gut microbiota by nutrients with prebiotic properties: consequences for host health in the context of obesity and metabolic syndrome. Microb Cell Fact 2011; 10 Suppl 1: S10.

30. lacobellis G, Leonetti F. Epicardial adipose tissue and insulin resistance in obese subjects. J Clin Endocrinol Metab 2005; 90: 6300-6302.

31. Wang CP, Hsu HL, Hung WC, et al. Increased epicardial adipose tissue (EAT) volume in type 2 diabetes mellitus and association with metabolic syndrome and severity of coronary atherosclerosis. Clin Endocrinol (Oxf) 2009; 70: 876-882.

32. Hammer S, van der Meer RW, Lamb HJ, et al. Short-term flexibility of myocardial triglycerides and diastolic function in patients with type 2 diabetes mellitus. Am J Physiol Endocrinol Metab 2008; 295: E714-718.

33. Kim MK, Tanaka K, Kim MJ, et al. Comparison of epicardial, abdominal and regional fat compartments in response to weight loss. Nutr Metab Cardiovasc Dis 2009; 19: 760-766.

34. lacobellis G, Singh N, Wharton S, Sharma AM. Substantial changes in epicardial fat thickness after weight loss in severely obese subjects. Obesity (Silver Spring) 2008; 16: 1693-1697.

35. Hammer S, van der Meer RW, Lamb HJ, et al. Progressive caloric restriction induces dose-dependent changes in myocardial triglyceride content and diastolic function in healthy men. J Clin Endocrinol Metab 2008; 93: 497-503.

36. de Vries AP, Ruggenenti $P$, Ruan $X Z$, et al. Fatty kidney: emerging role of ectopic lipid in obesity-related renal disease. Lancet Diabetes Endocrinol 2014; 2: 417-426.

37. Guebre-Egziabher F, Alix PM, Koppe L, et al. Ectopic lipid accumulation: A potential cause for metabolic disturbances and a contributor to the alteration of kidney function. Biochimie 2013; 95: 1971-1979.

38. Wei J, Ferron M, Clarke CJ, et al. Bone-specific insulin resistance disrupts wholebody glucose homeostasis via decreased osteocalcin activation. J Clin Invest 2014; 124: 1-13.

39. Veldhuis-Vlug AG, Fliers E, Bisschop PH. Bone as a regulator of glucose metabolism. Neth J Med 2013; 71: 396-400.

40. Gimble JM, Nuttall ME. The relationship between adipose tissue and bone metabolism. Clin Biochem 2012; 45: 874-879.

41. Frayn KN. Adipose tissue as a buffer for daily lipid flux. Diabetologia 2002; 45: 12011210. 
42. Evans K, Burdge GC, Wootton SA, Clark ML, Frayn KN. Regulation of dietary fatty acid entrapment in subcutaneous adipose tissue and skeletal muscle. Diabetes 2002; 51: 2684-2690.

43. Frayn KN, Coppack SW, Fielding BA, Humphreys SM. Coordinated regulation of hormone-sensitive lipase and lipoprotein lipase in human adipose tissue in vivo: implications for the control of fat storage and fat mobilization. Adv Enzyme Regul 1995; 35: 163-178.

44. Yost TJ, Jensen DR, Haugen BR, Eckel RH. Effect of dietary macronutrient composition on tissue-specific lipoprotein lipase activity and insulin action in normalweight subjects. Am J Clin Nutr 1998; 68: 296-302.

45. Brunzell JD, Schwartz RS, Eckel RH, Goldberg AP. Insulin and adipose tissue lipoprotein lipase activity in humans. Int $J$ Obes 1981; 5: 685-694.

46. Goldberg IJ, Eckel $\mathrm{RH}$, Abumrad NA. Regulation of fatty acid uptake into tissues: lipoprotein lipase- and CD36-mediated pathways. J Lipid Res 2009; 50 Suppl: S8690.

47. Ong JM, Kern PA. Effect of feeding and obesity on lipoprotein lipase activity, immunoreactive protein, and messenger RNA levels in human adipose tissue. $J$ Clin Invest 1989; 84: 305-311.

48. Olivecrona $T$, Bergo M, Hultin M, Olivecrona $G$. Nutritional regulation of lipoprotein lipase. Can J Cardiol 1995; 11 Suppl G: 73G-78G.

49. Ong JM, Simsolo RB, Saffari B, Kern PA. The regulation of lipoprotein lipase gene expression by dexamethasone in isolated rat adipocytes. Endocrinology 1992; 130: 2310-2316.

50. Koliwad SK, Kuo T, Shipp LE, et al. Angiopoietin-like 4 (ANGPTL4, fasting-induced adipose factor) is a direct glucocorticoid receptor target and participates in glucocorticoid-regulated triglyceride metabolism. J Biol Chem 2009; 284: 2559325601.

51. Fried SK, Russell CD, Grauso NL, Brolin RE. Lipoprotein lipase regulation by insulin and glucocorticoid in subcutaneous and omental adipose tissues of obese women and men. J Clin Invest 1993; 92: 2191-2198.

52. Ottosson $M$, Marin $\mathrm{P}$, Karason $\mathrm{K}$, Elander A, Bjorntorp $\mathrm{P}$. Blockade of the glucocorticoid receptor with RU 486: effects in vitro and in vivo on human adipose tissue lipoprotein lipase activity. Obes Res 1995; 3: 233-240.

53. Kim SJ, Nian C, McIntosh CH. GIP increases human adipocyte LPL expression through CREB and TORC2-mediated trans-activation of the LPL gene. J Lipid Res 2010; 51: 3145-3157.

54. Kim SJ, Nian C, Mclntosh CH. Resistin knockout mice exhibit impaired adipocyte glucose-dependent insulinotropic polypeptide receptor (GIPR) expression. Diabetes 2013; 62: 471-477.

55. Beigneux AP, Davies BS, Gin P, et al. Glycosylphosphatidylinositol-anchored highdensity lipoprotein-binding protein 1 plays a critical role in the lipolytic processing of chylomicrons. Cell Metab 2007; 5: 279-291.

56. Davies BS, Beigneux AP, Barnes $\mathrm{RH}$, 2nd, et al. GPIHBP1 is responsible for the entry of lipoprotein lipase into capillaries. Cell Metab 2010; 12: 42-52.

57. Young SG, Davies BS, Voss CV, et al. GPIHBP1, an endothelial cell transporter for lipoprotein lipase. J Lipid Res 2011; 52: 1869-1884.

58. Davies BS, Waki H, Beigneux AP, et al. The expression of GPIHBP1, an endothelial cell binding site for lipoprotein lipase and chylomicrons, is induced by peroxisome proliferator-activated receptor-gamma. Mol Endocrinol 2008; 22: 2496-2504.

59. Pei-Ling Chiu A, Wang F, Lal N, et al. Endothelial cells respond to hyperglycemia by increasing the LPL transporter GPIHBP1. Am J Physiol Endocrinol Metab 2014; 306: E1274-1283. 
60. Adeyo O, Goulbourne CN, Bensadoun A, Beigneux AP, Fong LG, Young SG. Glycosylphosphatidylinositol-anchored high-density lipoprotein-binding protein 1 and the intravascular processing of triglyceride-rich lipoproteins. J Intern Med 2012; 272: 528-540.

61. Weinstein MM, Goulbourne CN, Davies BS, et al. Reciprocal metabolic perturbations in the adipose tissue and liver of GPIHBP1-deficient mice. Arterioscler Thromb Vasc Biol 2012; 32: 230-235.

62. Kersten S. Physiological regulation of lipoprotein lipase. Biochim Biophys Acta 2014; 1841: 919-933.

63. Jonker JT, Smit JW, Hammer S, et al. Dietary modulation of plasma angiopoietin-like protein 4 concentrations in healthy volunteers and in patients with type 2 diabetes. Am J Clin Nutr 2013; 97: 255-260.

64. Brands M, Sauerwein HP, Ackermans MT, Kersten S, Serlie MJ. Omega-3 long-chain fatty acids strongly induce angiopoietin-like 4 in humans. J Lipid Res 2013; 54: 615621.

65. Amri EZ, Teboul L, Vannier C, Grimaldi PA, Ailhaud G. Fatty acids regulate the expression of lipoprotein lipase gene and activity in preadipose and adipose cells. Biochem J 1996; 314 ( Pt 2): 541-546.

66. Nagashima K, Lopez C, Donovan D, et al. Effects of the PPARgamma agonist pioglitazone on lipoprotein metabolism in patients with type 2 diabetes mellitus. J Clin Invest 2005; 115: 1323-1332.

67. Sadur $\mathrm{CN}$, Yost TJ, Eckel $\mathrm{RH}$. Insulin responsiveness of adipose tissue lipoprotein lipase is delayed but preserved in obesity. J Clin Endocrinol Metab 1984; 59: 11761182.

68. Riemens SC, Sluiter WJ, Dullaart RP. Enhanced escape of non-esterified fatty acids from tissue uptake: its role in impaired insulin-induced lowering of total rate of appearance in obesity and Type II diabetes mellitus. Diabetologia 2000; 43: 416-426.

69. McQuaid SE, Hodson L, Neville MJ, et al. Downregulation of adipose tissue fatty acid trafficking in obesity: a driver for ectopic fat deposition? Diabetes 2011; 60: 47-55.

70. Panarotto D, Remillard P, Bouffard L, Maheux P. Insulin resistance affects the regulation of lipoprotein lipase in the postprandial period and in an adipose tissuespecific manner. Eur J Clin Invest 2002; 32: 84-92.

71. Coppack SW, Evans RD, Fisher RM, et al. Adipose tissue metabolism in obesity: lipase action in vivo before and after a mixed meal. Metabolism 1992; 41: 264-272.

72. Potts JL, Coppack SW, Fisher RM, Humphreys SM, Gibbons GF, Frayn KN. Impaired postprandial clearance of triacylglycerol-rich lipoproteins in adipose tissue in obese subjects. Am J Physiol 1995; 268: E588-594.

73. Annuzzi G, Giacco R, Patti L, et al. Postprandial chylomicrons and adipose tissue lipoprotein lipase are altered in type 2 diabetes independently of obesity and wholebody insulin resistance. Nutr Metab Cardiovasc Dis 2008; 18: 531-538.

74. Frayn KN, Shadid S, Hamlani R, et al. Regulation of fatty acid movement in human adipose tissue in the postabsorptive-to-postprandial transition. Am J Physiol 1994; 266: E308-317.

75. Ruge T, Hodson L, Cheeseman J, et al. Fasted to fed trafficking of Fatty acids in human adipose tissue reveals a novel regulatory step for enhanced fat storage. $J$ Clin Endocrinol Metab 2009; 94: 1781-1788.

76. Brasaemle DL. Thematic review series: adipocyte biology. The perilipin family of structural lipid droplet proteins: stabilization of lipid droplets and control of lipolysis. $J$ Lipid Res 2007; 48: 2547-2559.

77. Glatz JF, Luiken JJ, Bonen A. Involvement of membrane-associated proteins in the acute regulation of cellular fatty acid uptake. $J$ Mol Neurosci 2001; 16: 123-132; discussion 151-127. 
78. Schwenk RW, Luiken JJ, Bonen A, Glatz JF. Regulation of sarcolemmal glucose and fatty acid transporters in cardiac disease. Cardiovasc Res 2008; 79: 249-258.

79. Buque X, Cano A, Miquilena-Colina ME, Garcia-Monzon C, Ochoa B, Aspichueta P. High insulin levels are required for FAT/CD36 plasma membrane translocation and enhanced fatty acid uptake in obese Zucker rat hepatocytes. Am J Physiol Endocrinol Metab 2012; 303: E504-514.

80. Chabowski A, Coort SL, Calles-Escandon J, et al. Insulin stimulates fatty acid transport by regulating expression of FAT/CD36 but not FABPpm. Am J Physiol Endocrinol Metab 2004; 287: E781-789.

81. Luiken JJ, Dyck DJ, Han XX, et al. Insulin induces the translocation of the fatty acid transporter FAT/CD36 to the plasma membrane. Am J Physiol Endocrinol Metab 2002; 282: E491-495.

82. Bonen A, Tandon NN, Glatz JF, Luiken JJ, Heigenhauser GJ. The fatty acid transporter FAT/CD36 is upregulated in subcutaneous and visceral adipose tissues in human obesity and type 2 diabetes. Int J Obes (Lond) 2006; 30: 877-883.

83. Pietka TA, Schappe T, Conte $\mathrm{C}$, et al. Adipose and muscle tissue profile of CD36 transcripts in obese subjects highlights the role of CD36 in fatty acid homeostasis and insulin resistance. Diabetes Care 2014; 37: 1990-1997.

84. Bush NC, Triay JM, Gathaiya NW, Hames KC, Jensen MD. Contribution of very lowdensity lipoprotein triglyceride fatty acids to postabsorptive free fatty acid flux in obese humans. Metabolism 2014; 63: 137-140.

85. Lafontan M, Langin D. Lipolysis and lipid mobilization in human adipose tissue. Prog Lipid Res 2009; 48: 275-297.

86. Sengenes C, Berlan M, De Glisezinski I, Lafontan M, Galitzky J. Natriuretic peptides: a new lipolytic pathway in human adipocytes. FASEB J 2000; 14: 1345-1351.

87. Sengenes $\mathrm{C}$, Bouloumie $\mathrm{A}$, Hauner $\mathrm{H}$, et al. Involvement of a cGMP-dependent pathway in the natriuretic peptide-mediated hormone-sensitive lipase phosphorylation in human adipocytes. $J$ Biol Chem 2003; 278: 48617-48626.

88. Birkenfeld AL, Boschmann M, Moro C, et al. Lipid mobilization with physiological atrial natriuretic peptide concentrations in humans. J Clin Endocrinol Metab 2005; 90: 3622-3628.

89. Jocken JW, Blaak EE. Catecholamine-induced lipolysis in adipose tissue and skeletal muscle in obesity. Physiol Behav 2008; 94: 219-230.

90. Zechner R, Zimmermann R, Eichmann TO, et al. FAT SIGNALS--lipases and lipolysis in lipid metabolism and signaling. Cell Metab 2012; 15: 279-291.

91. Jocken JW, Langin D, Smit E, et al. Adipose triglyceride lipase and hormonesensitive lipase protein expression is decreased in the obese insulin-resistant state. $J$ Clin Endocrinol Metab 2007; 92: 2292-2299.

92. Bickerton AS, Roberts R, Fielding BA, et al. Adipose tissue fatty acid metabolism in insulin-resistant men. Diabetologia 2008; 51: 1466-1474.

93. Groop LC, Bonadonna RC, Simonson DC, Petrides AS, Shank M, DeFronzo RA. Effect of insulin on oxidative and nonoxidative pathways of free fatty acid metabolism in human obesity. Am J Physiol 1992; 263: E79-84.

94. Langin D, Dicker A, Tavernier G, et al. Adipocyte lipases and defect of lipolysis in human obesity. Diabetes 2005; 54: 3190-3197.

95. Ryden $\mathrm{M}$, Jocken J, van Harmelen V, et al. Comparative studies of the role of hormone-sensitive lipase and adipose triglyceride lipase in human fat cell lipolysis. Am J Physiol Endocrinol Metab 2007; 292: E1847-1855.

96. Ray H, Pinteur C, Frering V, Beylot M, Large V. Depot-specific differences in perilipin and hormone-sensitive lipase expression in lean and obese. Lipids Health Dis 2009; 8: 58.

97. Wang $\mathrm{Y}$, Sullivan $\mathrm{S}$, Trujillo $\mathrm{M}$, et al. Perilipin expression in human adipose tissues: effects of severe obesity, gender, and depot. Obes Res 2003; 11: 930-936. 
98. Mottagui-Tabar S, Ryden M, Lofgren $\mathrm{P}$, et al. Evidence for an important role of perilipin in the regulation of human adipocyte lipolysis. Diabetologia 2003; 46: 789797.

99. Kern PA, Di Gregorio G, Lu T, Rassouli N, Ranganathan G. Perilipin expression in human adipose tissue is elevated with obesity. J Clin Endocrinol Metab 2004; 89: 1352-1358.

100. Claus TH, Lowe DB, Liang Y, et al. Specific inhibition of hormone-sensitive lipase improves lipid profile while reducing plasma glucose. J Pharmacol Exp Ther 2005; 315: 1396-1402.

101. Girousse A, Tavernier G, Valle C, et al. Partial inhibition of adipose tissue lipolysis improves glucose metabolism and insulin sensitivity without alteration of fat mass. PLoS Biol 2013; 11: e1001485.

102. Mayer N, Schweiger M, Romauch M, et al. Development of small-molecule inhibitors targeting adipose triglyceride lipase. Nat Chem Biol 2013; 9: 785-787.

103. Nagy HM, Paar M, Heier C, et al. Adipose triglyceride lipase activity is inhibited by long-chain acyl-coenzyme A. Biochim Biophys Acta 2014; 1841: 588-594.

104. Wang TJ, Larson MG, Levy D, et al. Impact of obesity on plasma natriuretic peptide levels. Circulation 2004; 109: 594-600.

105. Moro C, Lafontan M. Natriuretic peptides and cGMP signaling control of energy homeostasis. Am J Physiol Heart Circ Physiol 2013; 304: H358-368.

106. Mund RA, Frishman WH. Brown adipose tissue thermogenesis: beta3adrenoreceptors as a potential target for the treatment of obesity in humans. Cardiol Rev 2013; 21: 265-269.

107. van Baak MA, Hul GB, Toubro S, et al. Acute effect of L-796568, a novel beta 3adrenergic receptor agonist, on energy expenditure in obese men. Clin Pharmacol Ther 2002; 71: 272-279.

108. Larsen TM, Toubro S, van Baak MA, et al. Effect of a 28-d treatment with L-796568, a novel beta(3)-adrenergic receptor agonist, on energy expenditure and body composition in obese men. Am J Clin Nutr 2002; 76: 780-788.

109. Singh R, Kaushik S, Wang $\mathrm{Y}$, et al. Autophagy regulates lipid metabolism. Nature 2009; 458: 1131-1135.

110. Zhang Y, Goldman S, Baerga R, Zhao Y, Komatsu M, Jin S. Adipose-specific deletion of autophagy-related gene 7 (atg7) in mice reveals a role in adipogenesis. Proc Natl Acad Sci U S A 2009; 106: 19860-19865.

111. Lizaso $\mathrm{A}$, Tan $\mathrm{KT}$, Lee $\mathrm{YH}$. beta-adrenergic receptor-stimulated lipolysis requires the RAB7-mediated autolysosomal lipid degradation. Autophagy 2013; 9: 1228-1243.

112. Zhang Y, Zeng X, Jin S. Autophagy in adipose tissue biology. Pharmacol Res 2012; 66: 505-512.

113. Maixner N, Kovsan J, Harman-Boehm I, Bluher M, Bashan N, Rudich A. Autophagy in adipose tissue. Obes Facts 2012; 5: 710-721.

114. Bluher M. Adipose tissue dysfunction contributes to obesity related metabolic diseases. Best Pract Res Clin Endocrinol Metab 2013; 27: 163-177.

115. Kosacka J, Kern M, Kloting N, et al. Autophagy in adipose tissue of patients with obesity and type 2 diabetes. Mol Cell Endocrinol 2015;

116. Nunez CE, Rodrigues VS, Gomes FS, et al. Defective regulation of adipose tissue autophagy in obesity. Int J Obes (Lond) 2013; 37: 1473-1480.

117. Khor VK, Shen WJ, Kraemer FB. Lipid droplet metabolism. Curr Opin Clin Nutr Metab Care 2013; 16: 632-637.

118. Wilfling F, Haas JT, Walther TC, Jr RV. Lipid droplet biogenesis. Curr Opin Cell Biol 2014; 29C: 39-45.

119. Gonzalez-Baro MR, Lewin TM, Coleman RA. Regulation of Triglyceride Metabolism. II. Function of mitochondrial GPAT1 in the regulation of triacylglycerol biosynthesis and insulin action. Am J Physiol Gastrointest Liver Physiol 2007; 292: G1195-1199. 
120. Coleman RA, Lewin TM, Muoio DM. Physiological and nutritional regulation of enzymes of triacylglycerol synthesis. Annu Rev Nutr 2000; 20: 77-103.

121. Stone SJ, Levin MC, Farese RV, Jr. Membrane topology and identification of key functional amino acid residues of murine acyl-CoA:diacylglycerol acyltransferase-2. $J$ Biol Chem 2006; 281: 40273-40282.

122. Gong J, Sun Z, Wu L, et al. Fsp27 promotes lipid droplet growth by lipid exchange and transfer at lipid droplet contact sites. J Cell Biol 2011; 195: 953-963.

123. Jambunathan S, Yin J, Khan W, Tamori Y, Puri V. FSP27 promotes lipid droplet clustering and then fusion to regulate triglyceride accumulation. PLoS One 2011; 6: e28614.

124. Puri V, Ranjit S, Konda S, et al. Cidea is associated with lipid droplets and insulin sensitivity in humans. Proc Natl Acad Sci U S A 2008; 105: 7833-7838.

125. Guilherme A, Virbasius JV, Puri V, Czech MP. Adipocyte dysfunctions linking obesity to insulin resistance and type 2 diabetes. Nat Rev Mol Cell Biol 2008; 9: 367-377.

126. Nishino N, Tamori Y, Tateya S, et al. FSP27 contributes to efficient energy storage in murine white adipocytes by promoting the formation of unilocular lipid droplets. J Clin Invest 2008; 118: 2808-2821.

127. Martinez-Botas J, Anderson JB, Tessier D, et al. Absence of perilipin results in leanness and reverses obesity in Lepr(db/db) mice. Nat Genet 2000; 26: 474-479.

128. Tansey JT, Sztalryd C, Gruia-Gray J, et al. Perilipin ablation results in a lean mouse with aberrant adipocyte lipolysis, enhanced leptin production, and resistance to dietinduced obesity. Proc Natl Acad Sci U S A 2001; 98: 6494-6499.

129. Krotkiewski M, Bjorntorp P, Sjostrom L, Smith U. Impact of obesity on metabolism in men and women. Importance of regional adipose tissue distribution. J Clin Invest 1983; 72: 1150-1162.

130. Konige $\mathrm{M}$, Wang $\mathrm{H}$, Sztalryd $\mathrm{C}$. Role of adipose specific lipid droplet proteins in maintaining whole body energy homeostasis. Biochim Biophys Acta 2014; 1842: 393401.

131. Tchoukalova YD, Votruba SB, Tchkonia T, Giorgadze N, Kirkland JL, Jensen MD. Regional differences in cellular mechanisms of adipose tissue gain with overfeeding. Proc Natl Acad Sci U S A 2010; 107: 18226-18231.

132. Mariman EC, Wang P. Adipocyte extracellular matrix composition, dynamics and role in obesity. Cell Mol Life Sci 2010; 67: 1277-1292.

133. Sun K, Kusminski CM, Scherer PE. Adipose tissue remodeling and obesity. J Clin Invest 2011; 121: 2094-2101.

134. Chun TH. Peri-adipocyte ECM remodeling in obesity and adipose tissue fibrosis. Adipocyte 2012; 1: 89-95.

135. Strissel KJ, Stancheva Z, Miyoshi H, et al. Adipocyte death, adipose tissue remodeling, and obesity complications. Diabetes 2007; 56: 2910-2918.

136. Divoux A, Tordjman J, Lacasa D, et al. Fibrosis in human adipose tissue: composition, distribution, and link with lipid metabolism and fat mass loss. Diabetes 2010; 59: 2817-2825.

137. Alligier M, Meugnier E, Debard C, et al. Subcutaneous adipose tissue remodeling during the initial phase of weight gain induced by overfeeding in humans. J Clin Endocrinol Metab 2012; 97: E183-192.

138. Kim JY, van de Wall E, Laplante $M$, et al. Obesity-associated improvements in metabolic profile through expansion of adipose tissue. J Clin Invest 2007; 117: 26212637.

139. Danforth E, Jr. Failure of adipocyte differentiation causes type II diabetes mellitus? Nat Genet 2000; 26: 13.

140. Cawthorn WP, Scheller EL, MacDougald OA. Adipose tissue stem cells: the great WAT hope. Trends Endocrinol Metab 2012; 23: 270-277. 
141. Macotela $\mathrm{Y}$, Emanuelli $\mathrm{B}$, Mori MA, et al. Intrinsic differences in adipocyte precursor cells from different white fat depots. Diabetes 2012; 61: 1691-1699.

142. Spalding KL, Arner E, Westermark PO, et al. Dynamics of fat cell turnover in humans. Nature 2008; 453: 783-787.

143. Wilson-Fritch L, Nicoloro S, Chouinard M, et al. Mitochondrial remodeling in adipose tissue associated with obesity and treatment with rosiglitazone. J Clin Invest 2004; 114: 1281-1289.

144. Kusminski CM, Scherer PE. Mitochondrial dysfunction in white adipose tissue. Trends Endocrinol Metab 2012; 23: 435-443.

145. Klimcakova E, Roussel B, Marquez-Quinones A, et al. Worsening of obesity and metabolic status yields similar molecular adaptations in human subcutaneous and visceral adipose tissue: decreased metabolism and increased immune response. $J$ Clin Endocrinol Metab 2011; 96: E73-82.

146. Chen L, Na R, Gu M, et al. Reduction of mitochondrial $\mathrm{H} 2 \mathrm{O} 2$ by overexpressing peroxiredoxin 3 improves glucose tolerance in mice. Aging Cell 2008; 7: 866-878.

147. Qatanani M, Tan $\mathrm{Y}$, Dobrin $\mathrm{R}$, et al. Inverse regulation of inflammation and mitochondrial function in adipose tissue defines extreme insulin sensitivity in morbidly obese patients. Diabetes 2013; 62: 855-863.

148. Bogacka I, Xie H, Bray GA, Smith SR. Pioglitazone induces mitochondrial biogenesis in human subcutaneous adipose tissue in vivo. Diabetes 2005; 54: 1392-1399.

149. Dahlman I, Forsgren M, Sjogren A, et al. Downregulation of electron transport chain genes in visceral adipose tissue in type 2 diabetes independent of obesity and possibly involving tumor necrosis factor-alpha. Diabetes 2006; 55: 1792-1799.

150. Yin X, Lanza IR, Swain JM, Sarr MG, Nair KS, Jensen MD. Adipocyte mitochondrial function is reduced in human obesity independent of fat cell size. J Clin Endocrinol Metab 2014; 99: E209-216.

151. Saris WH, Heymsfield SB. All metabolic roads lead to mitochondrial (dys)-function. Curr Opin Clin Nutr Metab Care 2007; 10: 661-663.

152. Mottillo EP, Bloch AE, Leff T, Granneman JG. Lipolytic products activate peroxisome proliferator-activated receptor (PPAR) alpha and delta in brown adipocytes to match fatty acid oxidation with supply. J Biol Chem 2012; 287: 25038-25048.

153. van Marken Lichtenbelt WD, Vanhommerig JW, Smulders NM, et al. Cold-activated brown adipose tissue in healthy men. N Engl J Med 2009; 360: 1500-1508.

154. Virtanen KA, Lidell ME, Orava J, et al. Functional brown adipose tissue in healthy adults. N Engl J Med 2009; 360: 1518-1525.

155. Saito M, Okamatsu-Ogura $Y$, Matsushita $M$, et al. High incidence of metabolically active brown adipose tissue in healthy adult humans: effects of cold exposure and adiposity. Diabetes 2009; 58: 1526-1531.

156. Vijgen GH, Bouvy ND, Teule GJ, et al. Increase in brown adipose tissue activity after weight loss in morbidly obese subjects. J Clin Endocrinol Metab 2012; 97: E12291233.

157. Chondronikola M, Volpi E, Borsheim E, et al. Brown Adipose Tissue Improves Whole Body Glucose Homeostasis and Insulin Sensitivity in Humans. Diabetes 2014;

158. Wu J, Bostrom P, Sparks LM, et al. Beige adipocytes are a distinct type of thermogenic fat cell in mouse and human. Cell 2012; 150: 366-376.

159. Ishibashi J, Seale P. Medicine. Beige can be slimming. Science 2010; 328: 11131114.

160. Petrovic N, Walden TB, Shabalina IG, Timmons JA, Cannon B, Nedergaard J. Chronic peroxisome proliferator-activated receptor gamma (PPARgamma) activation of epididymally derived white adipocyte cultures reveals a population of thermogenically competent, UCP1-containing adipocytes molecularly distinct from classic brown adipocytes. J Biol Chem 2010; 285: 7153-7164. 
161. Walden TB, Hansen IR, Timmons JA, Cannon B, Nedergaard J. Recruited vs. nonrecruited molecular signatures of brown, "brite," and white adipose tissues. Am $J$ Physiol Endocrinol Metab 2012; 302: E19-31.

162. van Marken Lichtenbelt $\mathrm{W}$. Brown adipose tissue and the regulation of nonshivering thermogenesis. Curr Opin Clin Nutr Metab Care 2012; 15: 547-552.

163. Yoneshiro T, Aita S, Kawai Y, Iwanaga T, Saito M. Nonpungent capsaicin analogs (capsinoids) increase energy expenditure through the activation of brown adipose tissue in humans. Am J Clin Nutr 2012; 95: 845-850.

164. Rosen ED, Spiegelman BM. What we talk about when we talk about fat. Cell 2014; 156: 20-44.

165. Fisher FM, Kleiner S, Douris N, et al. FGF21 regulates PGC-1alpha and browning of white adipose tissues in adaptive thermogenesis. Genes Dev 2012; 26: 271-281.

166. Schulz TJ, Huang $P$, Huang TL, et al. Brown-fat paucity due to impaired BMP signalling induces compensatory browning of white fat. Nature 2013; 495: 379-383.

167. Cao H, Gerhold K, Mayers JR, Wiest MM, Watkins SM, Hotamisligil GS. Identification of a lipokine, a lipid hormone linking adipose tissue to systemic metabolism. Cell 2008; 134: 933-944.

168. Weisberg SP, McCann D, Desai M, Rosenbaum M, Leibel RL, Ferrante AW, Jr. Obesity is associated with macrophage accumulation in adipose tissue. $J$ Clin Invest 2003; 112: 1796-1808.

169. Xu H, Barnes GT, Yang Q, et al. Chronic inflammation in fat plays a crucial role in the development of obesity-related insulin resistance. J Clin Invest 2003; 112: 18211830.

170. Mraz M, Haluzik M. The role of adipose tissue immune cells in obesity and low-grade inflammation. J Endocrinol 2014; 222: R113-127.

171. Mathis D. Immunological goings-on in visceral adipose tissue. Cell Metab 2013; 17: 851-859.

172. Sun S, Ji Y, Kersten S, Qi L. Mechanisms of inflammatory responses in obese adipose tissue. Annu Rev Nutr 2012; 32: 261-286.

173. Lu M, Patsouris $\mathrm{D}$, Li P, et al. A new antidiabetic compound attenuates inflammation and insulin resistance in Zucker diabetic fatty rats. Am J Physiol Endocrinol Metab 2010; 298: E1036-1048.

174. Samuel VT, Petersen KF, Shulman Gl. Lipid-induced insulin resistance: unravelling the mechanism. Lancet 2010; 375: 2267-2277.

175. Shi H, Kokoeva MV, Inouye K, Tzameli I, Yin H, Flier JS. TLR4 links innate immunity and fatty acid-induced insulin resistance. J Clin Invest 2006; 116: 3015-3025.

176. Caricilli AM, Saad MJ. The role of gut microbiota on insulin resistance. Nutrients 2013; 5: 829-851.

177. Chambrier C, Bastard JP, Rieusset J, et al. Eicosapentaenoic acid induces mRNA expression of peroxisome proliferator-activated receptor gamma. Obes Res 2002; 10: 518-525.

178. Bassaganya-Riera J, Misyak S, Guri AJ, Hontecillas R. PPAR gamma is highly expressed in F4/80(hi) adipose tissue macrophages and dampens adipose-tissue inflammation. Cell Immunol 2009; 258: 138-146.

179. Flamment M, Hajduch E, Ferre P, Foufelle F. New insights into ER stress-induced insulin resistance. Trends Endocrinol Metab 2012; 23: 381-390.

180. Sopasakis VR, Sandqvist M, Gustafson B, et al. High local concentrations and effects on differentiation implicate interleukin-6 as a paracrine regulator. Obes Res 2004; 12 : 454-460.

181. Petruschke T, Hauner H. Tumor necrosis factor-alpha prevents the differentiation of human adipocyte precursor cells and causes delipidation of newly developed fat cells. J Clin Endocrinol Metab 1993; 76: 742-747. 
182. Xing H, Northrop JP, Grove JR, Kilpatrick KE, Su JL, Ringold GM. TNF alphamediated inhibition and reversal of adipocyte differentiation is accompanied by suppressed expression of PPARgamma without effects on Pref-1 expression. Endocrinology 1997; 138: 2776-2783.

183. Prins JB, Niesler CU, Winterford CM, et al. Tumor necrosis factor-alpha induces apoptosis of human adipose cells. Diabetes 1997; 46: 1939-1944.

184. Hauner $\mathrm{H}$, Petruschke T, Russ M, Rohrig K, Eckel J. Effects of tumour necrosis factor alpha (TNF alpha) on glucose transport and lipid metabolism of newly-differentiated human fat cells in cell culture. Diabetologia 1995; 38: 764-771.

185. Souza SC, Palmer HJ, Kang YH, et al. TNF-alpha induction of lipolysis is mediated through activation of the extracellular signal related kinase pathway in 3T3-L1 adipocytes. J Cell Biochem 2003; 89: 1077-1086.

186. Zhang HH, Halbleib M, Ahmad F, Manganiello VC, Greenberg AS. Tumor necrosis factor-alpha stimulates lipolysis in differentiated human adipocytes through activation of extracellular signal-related kinase and elevation of intracellular cAMP. Diabetes 2002; 51: 2929-2935.

187. van Hall G, Steensberg A, Sacchetti M, et al. Interleukin-6 stimulates lipolysis and fat oxidation in humans. J Clin Endocrinol Metab 2003; 88: 3005-3010.

188. Kahn BB, Flier JS. Obesity and insulin resistance. J Clin Invest 2000; 106: 473-481.

189. Dyck DJ, Heigenhauser GJ, Bruce CR. The role of adipokines as regulators of skeletal muscle fatty acid metabolism and insulin sensitivity. Acta Physiol (Oxf) 2006; 186: 5-16.

190. Yamauchi T, Kamon J, Ito $\mathrm{Y}$, et al. Cloning of adiponectin receptors that mediate antidiabetic metabolic effects. Nature 2003; 423: 762-769.

191. Yamauchi T, Kamon J, Minokoshi Y, et al. Adiponectin stimulates glucose utilization and fatty-acid oxidation by activating AMP-activated protein kinase. Nat Med 2002; 8: 1288-1295.

192. Ohashi K, Shibata R, Murohara T, Ouchi N. Role of anti-inflammatory adipokines in obesity-related diseases. Trends Endocrinol Metab 2014; 25: 348-355.

193. Wong GW, Krawczyk SA, Kitidis-Mitrokostas C, Revett T, Gimeno R, Lodish HF. Molecular, biochemical and functional characterizations of C1q/TNF family members: adipose-tissue-selective expression patterns, regulation by PPAR-gamma agonist, cysteine-mediated oligomerizations, combinatorial associations and metabolic functions. Biochem J 2008; 416: 161-177.

194. Wong GW, Krawczyk SA, Kitidis-Mitrokostas C, et al. Identification and characterization of CTRP9, a novel secreted glycoprotein, from adipose tissue that reduces serum glucose in mice and forms heterotrimers with adiponectin. FASEB $J$ 2009; 23: 241-258.

195. Peterson JM, Wei Z, Wong GW. C1q/TNF-related protein-3 (CTRP3), a novel adipokine that regulates hepatic glucose output. J Biol Chem 2010; 285: 3969139701.

196. Kopp A, Bala M, Buechler C, et al. C1q/TNF-related protein-3 represents a novel and endogenous lipopolysaccharide antagonist of the adipose tissue. Endocrinology 2010; 151: 5267-5278.

197. Wolfing B, Buechler C, Weigert J, et al. Effects of the new C1q/TNF-related protein (CTRP-3) "cartonectin" on the adipocytic secretion of adipokines. Obesity (Silver Spring) 2008; 16: 1481-1486.

198. Kim MJ, Lee W, Park EJ, Park SY. C1qTNF-related protein-6 increases the expression of interleukin-10 in macrophages. Mol Cells 2010; 30: 59-64.

199. Lee W, Kim MJ, Park EJ, Choi YJ, Park SY. C1qTNF-related protein-6 mediates fatty acid oxidation via the activation of the AMP-activated protein kinase. FEBS Lett 2010; 584: 968-972. 
200. Peterson JM, Wei Z, Seldin MM, Byerly MS, Aja S, Wong GW. CTRP9 transgenic mice are protected from diet-induced obesity and metabolic dysfunction. Am J Physiol Regul Integr Comp Physiol 2013; 305: R522-533.

201. Enomoto T, Ohashi K, Shibata R, et al. Adipolin/C1qdc2/CTRP12 protein functions as an adipokine that improves glucose metabolism. J Biol Chem 2011; 286: 3455234558.

202. Wei Z, Peterson JM, Lei X, et al. C1q/TNF-related protein-12 (CTRP12), a novel adipokine that improves insulin sensitivity and glycemic control in mouse models of obesity and diabetes. J Biol Chem 2012; 287: 10301-10315.

203. Yang RZ, Lee MJ, $\mathrm{Hu} \mathrm{H}$, et al. Identification of omentin as a novel depot-specific adipokine in human adipose tissue: possible role in modulating insulin action. $A m \mathrm{~J}$ Physiol Endocrinol Metab 2006; 290: E1253-1261.

204. de Souza Batista CM, Yang RZ, Lee MJ, et al. Omentin plasma levels and gene expression are decreased in obesity. Diabetes 2007; 56: 1655-1661.

205. Lamers D, Famulla S, Wronkowitz N, et al. Dipeptidyl peptidase 4 is a novel adipokine potentially linking obesity to the metabolic syndrome. Diabetes $2011 ; 60$ : 1917-1925.

206. Giannocco G, Oliveira KC, Crajoinas RO, et al. Dipeptidyl peptidase IV inhibition upregulates GLUT4 translocation and expression in heart and skeletal muscle of spontaneously hypertensive rats. Eur J Pharmacol 2013; 698: 74-86.

207. Sakamoto T, Takahashi N, Sawaragi Y, et al. Inflammation induced by RAW macrophages suppresses UCP1 mRNA induction via ERK activation in 10T1/2 adipocytes. Am J Physiol Cell Physiol 2013; 304: C729-738.

208. Cao X, Gao Z, Robert CE, et al. Pancreatic-derived factor (FAM3B), a novel islet cytokine, induces apoptosis of insulin-secreting beta-cells. Diabetes 2003; 52: 22962303.

209. Shimano M, Ouchi N, Walsh K. Cardiokines: recent progress in elucidating the cardiac secretome. Circulation 2012; 126: e327-332.

210. Fernandes J, Vogt J, Wolever TM. Intravenous acetate elicits a greater free fatty acid rebound in normal than hyperinsulinaemic humans. Eur J Clin Nutr 2012; 66: 10291034.

211. Aberdein N, Schweizer M, Ball D. Sodium acetate decreases phosphorylation of hormone sensitive lipase in isoproterenol-stimulated 3T3-L1 mature adipocytes. Adipocyte 2014; 3: 121-125.

212. Most J, Goossens GH, Jocken JW, Blaak EE. Short-term supplementation with a specific combination of dietary polyphenols increases energy expenditure and alters substrate metabolism in overweight subjects. Int J Obes (Lond) 2014; 38: 698-706.

213. Konings E, Timmers S, Boekschoten MV, et al. The effects of 30 days resveratrol supplementation on adipose tissue morphology and gene expression patterns in obese men. Int J Obes (Lond) 2014; 38: 470-473.

214. Goossens $\mathrm{GH}$, Bizzarri $\mathrm{A}$, Venteclef $\mathrm{N}$, et al. Increased adipose tissue oxygen tension in obese compared with lean men is accompanied by insulin resistance, impaired adipose tissue capillarization, and inflammation. Circulation 2011; 124: 6776.

215. Goossens GH, Blaak EE. Adipose tissue oxygen tension: implications for chronic metabolic and inflammatory diseases. Curr Opin Clin Nutr Metab Care 2012; 15: 539546.

216. Lee $\mathrm{P}$, Linderman JD, Smith $\mathrm{S}$, et al. Irisin and FGF21 are cold-induced endocrine activators of brown fat function in humans. Cell Metab 2014; 19: 302-309.

217. Nettleton JA, Jebb S, Riserus U, Koletzko B, Fleming J. Role of dietary fats in the prevention and treatment of the metabolic syndrome. Ann Nutr Metab 2014; 64: 167178. 
218. Riserus U, Willett WC, Hu FB. Dietary fats and prevention of type 2 diabetes. Prog Lipid Res 2009; 48: 44-51.

219. Storlien LH, Jenkins AB, Chisholm DJ, Pascoe WS, Khouri S, Kraegen EW. Influence of dietary fat composition on development of insulin resistance in rats. Relationship to muscle triglyceride and omega-3 fatty acids in muscle phospholipid. Diabetes 1991; 40: $280-289$.

220. Jebb SA, Lovegrove JA, Griffin BA, et al. Effect of changing the amount and type of fat and carbohydrate on insulin sensitivity and cardiovascular risk: the RISCK (Reading, Imperial, Surrey, Cambridge, and Kings) trial. Am J Clin Nutr 2010; 92: 748-758.

221. Tierney AC, McMonagle J, Shaw DI, et al. Effects of dietary fat modification on insulin sensitivity and on other risk factors of the metabolic syndrome--LIPGENE: a European randomized dietary intervention study. Int J Obes (Lond) 2011; 35: 800809.

222. Barber E, Sinclair AJ, Cameron-Smith D. Comparative actions of omega-3 fatty acids on in-vitro lipid droplet formation. Prostaglandins Leukot Essent Fatty Acids 2013; 89: 359-366.

223. Flachs P, Rossmeisl M, Kuda O, Kopecky J. Stimulation of mitochondrial oxidative capacity in white fat independent of UCP1: a key to lean phenotype. Biochim Biophys Acta 2013; 1831: 986-1003.

224. Flachs $\mathrm{P}$, Horakova $\mathrm{O}$, Brauner $\mathrm{P}$, et al. Polyunsaturated fatty acids of marine origin upregulate mitochondrial biogenesis and induce beta-oxidation in white fat. Diabetologia 2005; 48: 2365-2375.

225. Hensler M, Bardova K, Jilkova ZM, et al. The inhibition of fat cell proliferation by $n-3$ fatty acids in dietary obese mice. Lipids Health Dis 2011; 10: 128.

226. Ruzickova J, Rossmeisl M, Prazak T, et al. Omega-3 PUFA of marine origin limit dietinduced obesity in mice by reducing cellularity of adipose tissue. Lipids 2004; 39: 1177-1185.

227. Summers LK, Fielding BA, Bradshaw HA, et al. Substituting dietary saturated fat with polyunsaturated fat changes abdominal fat distribution and improves insulin sensitivity. Diabetologia 2002; 45: 369-377.

228. Bjermo H, Iggman D, Kullberg J, et al. Effects of $n-6$ PUFAs compared with SFAs on liver fat, lipoproteins, and inflammation in abdominal obesity: a randomized controlled trial. Am J Clin Nutr 2012; 95: 1003-1012.

229. Weigert $\mathrm{C}$, Brodbeck $\mathrm{K}$, Staiger $\mathrm{H}$, et al. Palmitate, but not unsaturated fatty acids, induces the expression of interleukin- 6 in human myotubes through proteasomedependent activation of nuclear factor-kappaB. J Biol Chem 2004; 279: 23942 23952.

230. Ajuwon KM, Spurlock ME. Palmitate activates the NF-kappaB transcription factor and induces IL-6 and TNFalpha expression in 3T3-L1 adipocytes. J Nutr 2005; 135: 18411846.

231. Baer DJ, Judd JT, Clevidence BA, Tracy RP. Dietary fatty acids affect plasma markers of inflammation in healthy men fed controlled diets: a randomized crossover study. Am J Clin Nutr 2004; 79: 969-973.

232. Thies F, Miles EA, Nebe-von-Caron G, et al. Influence of dietary supplementation with long-chain $n-3$ or $n-6$ polyunsaturated fatty acids on blood inflammatory cell populations and functions and on plasma soluble adhesion molecules in healthy adults. Lipids 2001; 36: 1183-1193.

233. Itariu BK, Zeyda M, Hochbrugger EE, et al. Long-chain n-3 PUFAs reduce adipose tissue and systemic inflammation in severely obese nondiabetic patients: a randomized controlled trial. Am J Clin Nutr 2012; 96: 1137-1149.

234. Reaven GM. Pathophysiology of insulin resistance in human disease. Physiol Rev 1995; 75: 473-486. 
235. McGarry JD. Banting lecture 2001: dysregulation of fatty acid metabolism in the etiology of type 2 diabetes. Diabetes 2002; 51: 7-18.

236. Kowal RC, Herz J, Goldstein JL, Esser V, Brown MS. Low density lipoprotein receptor-related protein mediates uptake of cholesteryl esters derived from apoprotein E-enriched lipoproteins. Proc Natl Acad Sci U S A 1989; 86: 5810-5814.

237. Neuschwander-Tetri BA. Hepatic lipotoxicity and the pathogenesis of nonalcoholic steatohepatitis: the central role of nontriglyceride fatty acid metabolites. Hepatology 2010; 52: 774-788.

238. Kabir M, Catalano KJ, Ananthnarayan S, et al. Molecular evidence supporting the portal theory: a causative link between visceral adiposity and hepatic insulin resistance. Am J Physiol Endocrinol Metab 2005; 288: E454-461.

239. Jensen MD. Role of body fat distribution and the metabolic complications of obesity. J Clin Endocrinol Metab 2008; 93: S57-63.

240. Catalano KJ, Stefanovski D, Bergman RN. Critical role of the mesenteric depot versus other intra-abdominal adipose depots in the development of insulin resistance in young rats. Diabetes 2010; 59: 1416-1423.

241. Rytka JM, Wueest S, Schoenle EJ, Konrad D. The portal theory supported by venous drainage-selective fat transplantation. Diabetes 2011; 60: 56-63.

242. Fromenty B, Robin MA, Igoudjil A, Mansouri A, Pessayre D. The ins and outs of mitochondrial dysfunction in NASH. Diabetes Metab 2004; 30: 121-138.

243. Ferre P, Foufelle F. Hepatic steatosis: a role for de novo lipogenesis and the transcription factor SREBP-1c. Diabetes Obes Metab 2010; 12 Suppl 2: 83-92.

244. Wiggins D, Gibbons GF. The lipolysis/esterification cycle of hepatic triacylglycerol. Its role in the secretion of very-low-density lipoprotein and its response to hormones and sulphonylureas. Biochem J 1992; 284 ( Pt 2): 457-462.

245. Kissebah AH, Alfarsi S, Adams PW, Wynn V. Role of insulin resistance in adipose tissue and liver in the pathogenesis of endogenous hypertriglyceridaemia in man. Diabetologia 1976; 12: 563-571.

246. Abbasi F, McLaughlin T, Lamendola C, et al. Fasting remnant lipoprotein cholesterol and triglyceride concentrations are elevated in nondiabetic, insulin-resistant, female volunteers. J Clin Endocrinol Metab 1999; 84: 3903-3906.

247. Watanabe $\mathrm{N}$, Taniguchi $\mathrm{T}$, Taketoh $\mathrm{H}$, et al. Elevated remnant-like lipoprotein particles in impaired glucose tolerance and type 2 diabetic patients. Diabetes Care 1999; 22: 152-156.

248. Chan DC, Watts GF, Barrett PH, Mamo JC, Redgrave TG. Markers of triglyceriderich lipoprotein remnant metabolism in visceral obesity. Clin Chem 2002; 48: 278283.

249. Lewis GF. Fatty acid regulation of very low density lipoprotein production. Curr Opin Lipidol 1997; 8: 146-153.

250. Malmstrom R, Packard CJ, Caslake $M$, et al. Defective regulation of triglyceride metabolism by insulin in the liver in NIDDM. Diabetologia 1997; 40: 454-462.

251. Taskinen MR. Diabetic dyslipidaemia: from basic research to clinical practice. Diabetologia 2003; 46: 733-749.

252. Adeli K, Taghibiglou C, Van Iderstine SC, Lewis GF. Mechanisms of hepatic very low-density lipoprotein overproduction in insulin resistance. Trends Cardiovasc Med 2001; 11: 170-176.

253. Chatterjee C, Sparks DL. Hepatic lipase, high density lipoproteins, and hypertriglyceridemia. Am J Pathol 2011; 178: 1429-1433.

254. Despres JP, Ferland M, Moorjani S, et al. Role of hepatic-triglyceride lipase activity in the association between intra-abdominal fat and plasma HDL cholesterol in obese women. Arteriosclerosis 1989; 9: 485-492. 
255. Carr MC, Hokanson JE, Zambon A, et al. The contribution of intraabdominal fat to gender differences in hepatic lipase activity and low/high density lipoprotein heterogeneity. J Clin Endocrinol Metab 2001; 86: 2831-2837.

256. Pardina E, Baena-Fustegueras JA, Catalan R, et al. Increased expression and activity of hepatic lipase in the liver of morbidly obese adult patients in relation to lipid content. Obes Surg 2009; 19: 894-904.

257. Lewis GF, Murdoch S, Uffelman K, et al. Hepatic lipase mRNA, protein, and plasma enzyme activity is increased in the insulin-resistant, fructose-fed Syrian golden hamster and is partially normalized by the insulin sensitizer rosiglitazone. Diabetes 2004; 53: 2893-2900.

258. Sibley SD, Palmer JP, Hirsch IB, Brunzell JD. Visceral obesity, hepatic lipase activity, and dyslipidemia in type 1 diabetes. J Clin Endocrinol Metab 2003; 88: 3379-3384.

259. Miksztowicz V, Lucero D, Zago V, et al. Hepatic lipase activity is increased in nonalcoholic fatty liver disease beyond insulin resistance. Diabetes Metab Res Rev 2012; 28: 535-541.

260. Bradbury MW. Lipid metabolism and liver inflammation. I. Hepatic fatty acid uptake: possible role in steatosis. Am J Physiol Gastrointest Liver Physiol 2006; 290: G194198.

261. Falcon A, Doege $H$, Fluitt $A$, et al. FATP2 is a hepatic fatty acid transporter and peroxisomal very long-chain acyl-CoA synthetase. Am J Physiol Endocrinol Metab 2010; 299: E384-393.

262. Doege H, Grimm D, Falcon A, et al. Silencing of hepatic fatty acid transporter protein 5 in vivo reverses diet-induced non-alcoholic fatty liver disease and improves hyperglycemia. J Biol Chem 2008; 283: 22186-22192.

263. Coburn CT, Hajri T, Ibrahimi A, Abumrad NA. Role of CD36 in membrane transport and utilization of long-chain fatty acids by different tissues. J Mol Neurosci 2001; 16 : 117-121; discussion 151-117.

264. Su X, Abumrad NA. Cellular fatty acid uptake: a pathway under construction. Trends Endocrinol Metab 2009; 20: 72-77.

265. Doege H, Baillie RA, Ortegon AM, et al. Targeted deletion of FATP5 reveals multiple functions in liver metabolism: alterations in hepatic lipid homeostasis. Gastroenterology 2006; 130: 1245-1258.

266. Westerbacka J, Kolak M, Kiviluoto T, et al. Genes involved in fatty acid partitioning and binding, lipolysis, monocyte/macrophage recruitment, and inflammation are overexpressed in the human fatty liver of insulin-resistant subjects. Diabetes 2007; 56: $2759-2765$.

267. Nie B, Park HM, Kazantzis M, et al. Specific bile acids inhibit hepatic fatty acid uptake in mice. Hepatology 2012; 56: 1300-1310.

268. Fruhbeck G, Lopez M, Dieguez C. Role of caveolins in body weight and insulin resistance regulation. Trends Endocrinol Metab 2007; 18: 177-182.

269. Otsu K, Toya $\mathrm{Y}$, Oshikawa J, et al. Caveolin gene transfer improves glucose metabolism in diabetic mice. Am J Physiol Cell Physiol 2010; 298: C450-456.

270. Greco D, Kotronen A, Westerbacka J, et al. Gene expression in human NAFLD. Am J Physiol Gastrointest Liver Physiol 2008; 294: G1281-1287.

271. Goudriaan JR, Dahlmans VE, Teusink B, et al. CD36 deficiency increases insulin sensitivity in muscle, but induces insulin resistance in the liver in mice. J Lipid Res 2003; 44: 2270-2277.

272. Ong KT, Mashek MT, Bu SY, Mashek DG. Hepatic ATGL knockdown uncouples glucose intolerance from liver TAG accumulation. FASEB J 2013; 27: 313-321.

273. Voshol PJ, Haemmerle G, Ouwens DM, et al. Increased hepatic insulin sensitivity together with decreased hepatic triglyceride stores in hormone-sensitive lipasedeficient mice. Endocrinology 2003; 144: 3456-3462. 
274. Mulder $\mathrm{H}$, Sorhede-Winzell M, Contreras JA, et al. Hormone-sensitive lipase null mice exhibit signs of impaired insulin sensitivity whereas insulin secretion is intact. $J$ Biol Chem 2003; 278: 36380-36388.

275. Schweiger M, Schreiber R, Haemmerle G, et al. Adipose triglyceride lipase and hormone-sensitive lipase are the major enzymes in adipose tissue triacylglycerol catabolism. J Biol Chem 2006; 281: 40236-40241.

276. Wilson PA, Gardner SD, Lambie NM, Commans SA, Crowther DJ. Characterization of the human patatin-like phospholipase family. J Lipid Res 2006; 47: 1940-1949.

277. Wei E, Ben Ali Y, Lyon J, et al. Loss of TGH/Ces3 in mice decreases blood lipids, improves glucose tolerance, and increases energy expenditure. Cell Metab 2010; 11: 183-193.

278. Kienesberger PC, Oberer M, Lass A, Zechner R. Mammalian patatin domain containing proteins: a family with diverse lipolytic activities involved in multiple biological functions. J Lipid Res 2009; 50 Suppl: S63-68.

279. Wang CW, Lin HY, Shin SJ, et al. The PNPLA3 I148M polymorphism is associated with insulin resistance and nonalcoholic fatty liver disease in a normoglycaemic population. Liver Int 2011; 31: 1326-1331.

280. Palmer CN, Maglio C, Pirazzi C, et al. Paradoxical lower serum triglyceride levels and higher type 2 diabetes mellitus susceptibility in obese individuals with the PNPLA3 148M variant. PLoS One 2012; 7: e39362.

281. Kumashiro N, Yoshimura T, Cantley JL, et al. Role of patatin-like phospholipase domain-containing 3 on lipid-induced hepatic steatosis and insulin resistance in rats. Hepatology 2013; 57: 1763-1772.

282. Kawano $\mathrm{Y}$, Cohen DE. Mechanisms of hepatic triglyceride accumulation in nonalcoholic fatty liver disease. J Gastroenterol 2013; 48: 434-441.

283. Fujii $\mathrm{H}$, Ikura $\mathrm{Y}$, Arimoto $\mathrm{J}$, et al. Expression of perilipin and adipophilin in nonalcoholic fatty liver disease; relevance to oxidative injury and hepatocyte ballooning. J Atheroscler Thromb 2009; 16: 893-901.

284. Straub BK, Stoeffel P, Heid H, Zimbelmann R, Schirmacher P. Differential pattern of lipid droplet-associated proteins and de novo perilipin expression in hepatocyte steatogenesis. Hepatology 2008; 47: 1936-1946.

285. Inoue M, Ohtake T, Motomura W, et al. Increased expression of PPARgamma in high fat diet-induced liver steatosis in mice. Biochem Biophys Res Commun 2005; 336: 215-222.

286. Matsusue K, Kusakabe T, Noguchi T, et al. Hepatic steatosis in leptin-deficient mice is promoted by the PPARgamma target gene Fsp27. Cell Metab 2008; 7: 302-311.

287. Schadinger SE, Bucher NL, Schreiber BM, Farmer SR. PPARgamma2 regulates lipogenesis and lipid accumulation in steatotic hepatocytes. Am J Physiol Endocrinol Metab 2005; 288: E1195-1205.

288. Varela GM, Antwi DA, Dhir R, et al. Inhibition of ADRP prevents diet-induced insulin resistance. Am J Physiol Gastrointest Liver Physiol 2008; 295: G621-628.

289. Matsusue K. A physiological role for fat specific protein 27/cell death-inducing DFF45-like effector C in adipose and liver. Biol Pharm Bull 2010; 33: 346-350.

290. Yang Z, Klionsky DJ. Mammalian autophagy: core molecular machinery and signaling regulation. Curr Opin Cell Biol 2010; 22: 124-131.

291. Singh R, Cuervo AM. Lipophagy: connecting autophagy and lipid metabolism. Int $J$ Cell Biol 2012; 2012: 282041.

292. Liu HY, Han J, Cao SY, et al. Hepatic autophagy is suppressed in the presence of insulin resistance and hyperinsulinemia: inhibition of FoxO1-dependent expression of key autophagy genes by insulin. J Biol Chem 2009; 284: 31484-31492.

293. Yang L, Li P, Fu S, Calay ES, Hotamisligil GS. Defective hepatic autophagy in obesity promotes ER stress and causes insulin resistance. Cell Metab 2010; 11: 467478. 
294. Hellerstein MK. De novo lipogenesis in humans: metabolic and regulatory aspects. Eur J Clin Nutr 1999; 53 Suppl 1: S53-65.

295. Schwarz JM, Linfoot P, Dare D, Aghajanian K. Hepatic de novo lipogenesis in normoinsulinemic and hyperinsulinemic subjects consuming high-fat, lowcarbohydrate and low-fat, high-carbohydrate isoenergetic diets. Am J Clin Nutr 2003; 77: 43-50.

296. Jensen-Urstad AP, Semenkovich CF. Fatty acid synthase and liver triglyceride metabolism: housekeeper or messenger? Biochim Biophys Acta 2012; 1821: 747753.

297. Chakravarthy MV, Pan Z, Zhu Y, et al. "New" hepatic fat activates PPARalpha to maintain glucose, lipid, and cholesterol homeostasis. Cell Metab 2005; 1: 309-322.

298. Miyazaki M, Kim YC, Ntambi JM. A lipogenic diet in mice with a disruption of the stearoyl-CoA desaturase 1 gene reveals a stringent requirement of endogenous monounsaturated fatty acids for triglyceride synthesis. J Lipid Res 2001; 42: 10181024.

299. Smith SJ, Cases S, Jensen DR, et al. Obesity resistance and multiple mechanisms of triglyceride synthesis in mice lacking Dgat. Nat Genet 2000; 25: 87-90.

300. Chen HC, Smith SJ, Ladha Z, et al. Increased insulin and leptin sensitivity in mice lacking acyl CoA:diacylglycerol acyltransferase 1. J Clin Invest 2002; 109: 10491055.

301. Herman MA, Peroni OD, Villoria J, et al. A novel ChREBP isoform in adipose tissue regulates systemic glucose metabolism. Nature 2012; 484: 333-338.

302. Strable MS, Ntambi JM. Genetic control of de novo lipogenesis: role in diet-induced obesity. Crit Rev Biochem Mol Biol 2010; 45: 199-214.

303. Kersten S. Mechanisms of nutritional and hormonal regulation of lipogenesis. EMBO Rep 2001; 2: 282-286.

304. Postic C, Girard J. Contribution of de novo fatty acid synthesis to hepatic steatosis and insulin resistance: lessons from genetically engineered mice. J Clin Invest 2008; 118: 829-838.

305. Moczulski D, Majak I, Mamczur D. An overview of beta-oxidation disorders. Postepy Hig Med Dosw (Online) 2009; 63: 266-277.

306. Wanders RJ, Waterham HR. Biochemistry of mammalian peroxisomes revisited. Annu Rev Biochem 2006; 75: 295-332.

307. Schrader M, Fahimi HD. The peroxisome: still a mysterious organelle. Histochem Cell Biol 2008; 129: 421-440.

308. Kerner J, Hoppel C. Fatty acid import into mitochondria. Biochim Biophys Acta 2000; 1486: 1-17.

309. Park EA, Mynatt RL, Cook GA, Kashfi K. Insulin regulates enzyme activity, malonylCoA sensitivity and mRNA abundance of hepatic carnitine palmitoyltransferase-I. Biochem J 1995; 310 ( Pt 3): 853-858.

310. Verhoeven NM, Roe DS, Kok RM, Wanders RJ, Jakobs C, Roe CR. Phytanic acid and pristanic acid are oxidized by sequential peroxisomal and mitochondrial reactions in cultured fibroblasts. J Lipid Res 1998; 39: 66-74.

311. Hashimoto T. Peroxisomal beta-oxidation enzymes. Neurochem Res 1999; 24: 551563.

312. Reddy JK, Mannaerts GP. Peroxisomal lipid metabolism. Annu Rev Nutr 1994; 14: 343-370.

313. Jakobs BS, Wanders RJ. Fatty acid beta-oxidation in peroxisomes and mitochondria: the first, unequivocal evidence for the involvement of carnitine in shuttling propionylCoA from peroxisomes to mitochondria. Biochem Biophys Res Commun 1995; 213: 1035-1041.

314. Kim JA, Wei Y, Sowers JR. Role of mitochondrial dysfunction in insulin resistance. Circ Res 2008; 102: 401-414. 
315. Raffaella C, Francesca B, Italia F, Marina P, Giovanna L, Susanna I. Alterations in hepatic mitochondrial compartment in a model of obesity and insulin resistance. Obesity (Silver Spring) 2008; 16: 958-964.

316. Vial G, Dubouchaud H, Leverve XM. Liver mitochondria and insulin resistance. Acta Biochim Pol 2010; 57: 389-392.

317. Roduit R, Morin J, Masse F, et al. Glucose down-regulates the expression of the peroxisome proliferator-activated receptor-alpha gene in the pancreatic beta -cell. $J$ Biol Chem 2000; 275: 35799-35806.

318. Sidossis LS, Wolfe RR. Glucose and insulin-induced inhibition of fatty acid oxidation: the glucose-fatty acid cycle reversed. Am J Physiol 1996; 270: E733-738.

319. Sidossis LS, Mittendorfer B, Walser E, Chinkes D, Wolfe RR. Hyperglycemia-induced inhibition of splanchnic fatty acid oxidation increases hepatic triacylglycerol secretion. Am J Physiol 1998; 275: E798-805.

320. Koliaki C, Roden M. Hepatic energy metabolism in human diabetes mellitus, obesity and non-alcoholic fatty liver disease. Mol Cell Endocrinol 2013; 379: 35-42.

321. Lefebvre P, Cariou B, Lien F, Kuipers F, Staels B. Role of bile acids and bile acid receptors in metabolic regulation. Physiol Rev 2009; 89: 147-191.

322. Staels B, Fonseca VA. Bile acids and metabolic regulation: mechanisms and clinical responses to bile acid sequestration. Diabetes Care 2009; 32 Suppl 2: S237-245.

323. Thomas C, Pellicciari R, Pruzanski M, Auwerx J, Schoonjans K. Targeting bile-acid signalling for metabolic diseases. Nat Rev Drug Discov 2008; 7: 678-693.

324. Zhang Y, Edwards PA. FXR signaling in metabolic disease. FEBS Lett 2008; 582: 1018.

325. Clayton PT. Disorders of bile acid synthesis. J Inherit Metab Dis 2011; 34: 593-604.

326. Russell DW. The enzymes, regulation, and genetics of bile acid synthesis. Annu Rev Biochem 2003; 72: 137-174.

327. Inagaki T, Moschetta A, Lee YK, et al. Regulation of antibacterial defense in the small intestine by the nuclear bile acid receptor. Proc Natl Acad Sci U S A 2006; 103: 39203925.

328. Lee FY, Lee H, Hubbert ML, Edwards PA, Zhang Y. FXR, a multipurpose nuclear receptor. Trends Biochem Sci 2006; 31: 572-580.

329. Kast HR, Nguyen CM, Sinal CJ, et al. Farnesoid X-activated receptor induces apolipoprotein C-II transcription: a molecular mechanism linking plasma triglyceride levels to bile acids. Mol Endocrinol 2001; 15: 1720-1728.

330. Sirvent A, Claudel $T$, Martin $G$, et al. The farnesoid $X$ receptor induces very low density lipoprotein receptor gene expression. FEBS Lett 2004; 566: 173-177.

331. Anisfeld AM, Kast-Woelbern HR, Meyer ME, et al. Syndecan-1 expression is regulated in an isoform-specific manner by the farnesoid-X receptor. $J$ Biol Chem 2003; 278: 20420-20428.

332. Crouse JR, 3rd. Hypertriglyceridemia: a contraindication to the use of bile acid binding resins. Am J Med 1987; 83: 243-248.

333. Pineda Torra I, Claudel T, Duval C, Kosykh V, Fruchart JC, Staels B. Bile acids induce the expression of the human peroxisome proliferator-activated receptor alpha gene via activation of the farnesoid X receptor. Mol Endocrinol 2003; 17: 259-272.

334. Savkur RS, Bramlett KS, Michael LF, Burris TP. Regulation of pyruvate dehydrogenase kinase expression by the farnesoid X receptor. Biochem Biophys Res Commun 2005; 329: 391-396.

335. Cyphert HA, Ge X, Kohan AB, Salati LM, Zhang Y, Hillgartner FB. Activation of the farnesoid $X$ receptor induces hepatic expression and secretion of fibroblast growth factor 21. J Biol Chem 2012; 287: 25123-25138.

336. Pols TW, Noriega LG, Nomura M, Auwerx J, Schoonjans K. The bile acid membrane receptor TGR5 as an emerging target in metabolism and inflammation. $J$ Hepatol 2011; 54: 1263-1272. 
337. Furihata T, Hosokawa M, Satoh T, Chiba K. Synergistic role of specificity proteins and upstream stimulatory factor 1 in transactivation of the mouse carboxylesterase 2/microsomal acylcarnitine hydrolase gene promoter. Biochem J 2004; 384: 101-110.

338. Konig B, Koch A, Spielmann J, et al. Activation of PPARalpha and PPARgamma reduces triacylglycerol synthesis in rat hepatoma cells by reduction of nuclear SREBP-1. Eur J Pharmacol 2009; 605: 23-30.

339. Zhang $\mathrm{Y}$, Lei $\mathrm{T}$, Huang JF, et al. The link between fibroblast growth factor 21 and sterol regulatory element binding protein 1c during lipogenesis in hepatocytes. Mol Cell Endocrinol 2011; 342: 41-47.

340. D'Adamo E, Cali AM, Weiss R, et al. Central role of fatty liver in the pathogenesis of insulin resistance in obese adolescents. Diabetes Care 2010; 33: 1817-1822.

341. Rijzewijk LJ, van der Meer RW, Lubberink M, et al. Liver fat content in type 2 diabetes: relationship with hepatic perfusion and substrate metabolism. Diabetes 2010; 59: 2747-2754.

342. Robinson-Rechavi M, Escriva Garcia H, Laudet V. The nuclear receptor superfamily. J Cell Sci 2003; 116: 585-586.

343. Steffensen KR, Gustafsson JA. Putative metabolic effects of the liver $X$ receptor (LXR). Diabetes 2004; 53 Suppl 1: S36-42.

344. Plat J, Nichols JA, Mensink RP. Plant sterols and stanols: effects on mixed micellar composition and LXR (target gene) activation. J Lipid Res 2005; 46: 2468-2476.

345. Korach-Andre M, Parini P, Larsson L, Arner A, Steffensen KR, Gustafsson JA. Separate and overlapping metabolic functions of LXRalpha and LXRbeta in C57BI/6 female mice. Am J Physiol Endocrinol Metab 2010; 298: E167-178.

346. Grefhorst A, van Dijk TH, Hammer A, et al. Differential effects of pharmacological liver $X$ receptor activation on hepatic and peripheral insulin sensitivity in lean and ob/ob mice. Am J Physiol Endocrinol Metab 2005; 289: E829-838.

347. Baranowski M, Zabielski P, Blachnio-Zabielska AU, Harasim E, Chabowski A, Gorski J. Insulin-sensitizing effect of LXR agonist T0901317 in high-fat fed rats is associated with restored muscle GLUT4 expression and insulin-stimulated AS160 phosphorylation. Cell Physiol Biochem 2014; 33: 1047-1057.

348. Laurencikiene $\mathrm{J}$, Ryden $\mathrm{M}$. Liver $\mathrm{X}$ receptors and fat cell metabolism. Int $\mathrm{J}$ Obes (Lond) 2012; 36: 1494-1502.

349. Cha BS, Ciaraldi TP, Carter L, et al. Peroxisome proliferator-activated receptor (PPAR) gamma and retinoid $X$ receptor $(R X R)$ agonists have complementary effects on glucose and lipid metabolism in human skeletal muscle. Diabetologia 2001; 44: 444-452.

350. Mukherjee R, Davies PJ, Crombie DL, et al. Sensitization of diabetic and obese mice to insulin by retinoid $X$ receptor agonists. Nature 1997; 386: 407-410.

351. Cesario RM, Klausing K, Razzaghi $\mathrm{H}$, et al. The rexinoid LG100754 is a novel RXR:PPARgamma agonist and decreases glucose levels in vivo. Mol Endocrinol 2001; 15: 1360-1369.

352. Kakuta $\mathrm{H}$, Yakushiji N, Shinozaki R, et al. RXR Partial Agonist CBt-PMN Exerts Therapeutic Effects on Type 2 Diabetes without the Side Effects of RXR Full Agonists. ACS Med Chem Lett 2012; 3: 427-432.

353. Pols TW, Nomura M, Harach T, et al. TGR5 activation inhibits atherosclerosis by reducing macrophage inflammation and lipid loading. Cell Metab 2011; 14: 747-757.

354. Watanabe M, Houten SM, Mataki C, et al. Bile acids induce energy expenditure by promoting intracellular thyroid hormone activation. Nature 2006; 439: 484-489.

355. Houten SM, Watanabe M, Auwerx J. Endocrine functions of bile acids. EMBO J 2006; 25: 1419-1425.

356. Thomas C, Gioiello A, Noriega L, et al. TGR5-mediated bile acid sensing controls glucose homeostasis. Cell Metab 2009; 10: 167-177. 
357. Ockenga J, Valentini L, Schuetz T, et al. Plasma bile acids are associated with energy expenditure and thyroid function in humans. J Clin Endocrinol Metab 2012; 97: 535-542.

358. Prawitt J, Caron S, Staels B. Bile acid metabolism and the pathogenesis of type 2 diabetes. Curr Diab Rep 2011; 11: 160-166.

359. Haeusler RA, Astiarraga B, Camastra S, Accili D, Ferrannini E. Human insulin resistance is associated with increased plasma levels of 12alpha-hydroxylated bile acids. Diabetes 2013; 62: 4184-4191.

360. Andersen E, Karlaganis G, Sjovall J. Altered bile acid profiles in duodenal bile and urine in diabetic subjects. Eur J Clin Invest 1988; 18: 166-172.

361. Begley M, Hill C, Gahan CG. Bile salt hydrolase activity in probiotics. Appl Environ Microbiol 2006; 72: 1729-1738.

362. Batta AK, Salen G, Arora R, Shefer S, Batta M, Person A. Side chain conjugation prevents bacterial 7-dehydroxylation of bile acids. J Biol Chem 1990; 265: 1092510928.

363. Jones BV, Begley M, Hill C, Gahan CG, Marchesi JR. Functional and comparative metagenomic analysis of bile salt hydrolase activity in the human gut microbiome. Proc Natl Acad Sci U S A 2008; 105: 13580-13585.

364. Vrieze A, Out C, Fuentes S, et al. Impact of oral vancomycin on gut microbiota, bile acid metabolism, and insulin sensitivity. J Hepatol 2014; 60: 824-831.

365. Li T, Owsley E, Matozel M, Hsu P, Novak CM, Chiang JY. Transgenic expression of cholesterol 7alpha-hydroxylase in the liver prevents high-fat diet-induced obesity and insulin resistance in mice. Hepatology 2010; 52: 678-690.

366. Beysen C, Murphy EJ, Deines $\mathrm{K}$, et al. Effect of bile acid sequestrants on glucose metabolism, hepatic de novo lipogenesis, and cholesterol and bile acid kinetics in type 2 diabetes: a randomised controlled study. Diabetologia 2012; 55: 432-442.

367. Smushkin G, Sathananthan M, Piccinini F, et al. The effect of a bile acid sequestrant on glucose metabolism in subjects with type 2 diabetes. Diabetes 2013; 62: 10941101.

368. Kootte RS, Vrieze A, Holleman F, et al. The therapeutic potential of manipulating gut microbiota in obesity and type 2 diabetes mellitus. Diabetes Obes Metab 2012; 14: 112-120.

369. Sato $\mathrm{S}$, Nagai $\mathrm{H}$, Igarashi $\mathrm{Y}$. Effect of probiotics on serum bile acids in patients with ulcerative colitis. Hepatogastroenterology 2012; 59: 1804-1808.

370. Lazar MA. The humoral side of insulin resistance. Nat Med 2006; 12: 43-44.

371. Hotamisligil GS. Inflammation and endoplasmic reticulum stress in obesity and diabetes. Int J Obes (Lond) 2008; 32 Suppl 7: S52-54.

372. Pagliassotti MJ. Endoplasmic reticulum stress in nonalcoholic fatty liver disease. Annu Rev Nutr 2012; 32: 17-33.

373. Rao MS, Reddy JK. Peroxisomal beta-oxidation and steatohepatitis. Semin Liver Dis 2001; 21: 43-55.

374. Garcia-Monzon C, Martin-Perez E, lacono OL, et al. Characterization of pathogenic and prognostic factors of nonalcoholic steatohepatitis associated with obesity. $J$ Hepatol 2000; 33: 716-724.

375. Sanyal AJ, Campbell-Sargent C, Mirshahi F, et al. Nonalcoholic steatohepatitis: association of insulin resistance and mitochondrial abnormalities. Gastroenterology 2001; 120: 1183-1192.

376. Angulo P. Nonalcoholic fatty liver disease. N Engl J Med 2002; 346: 1221-1231.

377. Haubrich WS. Kupffer of Kupffer cells. Gastroenterology 2004; 127: 16.

378. Stefan N, Haring HU. The role of hepatokines in metabolism. Nat Rev Endocrinol 2013; 9: 144-152.

379. Lan F, Misu H, Chikamoto $\mathrm{K}$, et al. LECT2 functions as a hepatokine that links obesity to skeletal muscle insulin resistance. Diabetes 2014; 
380. Anderson N, Borlak J. Molecular mechanisms and therapeutic targets in steatosis and steatohepatitis. Pharmacol Rev 2008; 60: 311-357.

381. Pols TW, Noriega LG, Nomura M, Auwerx J, Schoonjans K. The bile acid membrane receptor TGR5: a valuable metabolic target. Dig Dis 2011; 29: 37-44.

382. Nieuwdorp M, Gilijamse PW, Pai N, Kaplan LM. Role of the microbiome in energy regulation and metabolism. Gastroenterology 2014; 146: 1525-1533.

383. Boden G, Lebed B, Schatz M, Homko C, Lemieux S. Effects of acute changes of plasma free fatty acids on intramyocellular fat content and insulin resistance in healthy subjects. Diabetes 2001; 50: 1612-1617.

384. Hegarty BD, Cooney GJ, Kraegen EW, Furler SM. Increased efficiency of fatty acid uptake contributes to lipid accumulation in skeletal muscle of high fat-fed insulinresistant rats. Diabetes 2002; 51: 1477-1484.

385. Schrauwen-Hinderling VB, Kooi ME, Hesselink MK, et al. Intramyocellular lipid content and molecular adaptations in response to a 1-week high-fat diet. Obes Res 2005; 13: 2088-2094.

386. Bachmann OP, Dahl DB, Brechtel K, et al. Effects of intravenous and dietary lipid challenge on intramyocellular lipid content and the relation with insulin sensitivity in humans. Diabetes 2001; 50: 2579-2584.

387. van Loon LJ. Use of intramuscular triacylglycerol as a substrate source during exercise in humans. J Appl Physiol (1985) 2004; 97: 1170-1187.

388. Schrauwen-Hinderling VB, Kooi ME, Hesselink MK, et al. Impaired in vivo mitochondrial function but similar intramyocellular lipid content in patients with type 2 diabetes mellitus and BMI-matched control subjects. Diabetologia 2007; 50: 113-120.

389. Goodpaster BH, He J, Watkins S, Kelley DE. Skeletal muscle lipid content and insulin resistance: evidence for a paradox in endurance-trained athletes. J Clin Endocrinol Metab 2001; 86: 5755-5761.

390. van Loon LJ, Goodpaster BH. Increased intramuscular lipid storage in the insulinresistant and endurance-trained state. Pflugers Arch 2006; 451: 606-616.

391. van Loon LJ, Koopman R, Manders R, van der Weegen W, van Kranenburg GP, Keizer HA. Intramyocellular lipid content in type 2 diabetes patients compared with overweight sedentary men and highly trained endurance athletes. Am J Physiol Endocrinol Metab 2004; 287: E558-565.

392. Anderson EJ, Lustig ME, Boyle KE, et al. Mitochondrial $\mathrm{H} 2 \mathrm{O} 2$ emission and cellular redox state link excess fat intake to insulin resistance in both rodents and humans. $J$ Clin Invest 2009; 119: 573-581.

393. Tarnopolsky MA, Rennie CD, Robertshaw HA, Fedak-Tarnopolsky SN, Devries MC, Hamadeh MJ. Influence of endurance exercise training and sex on intramyocellular lipid and mitochondrial ultrastructure, substrate use, and mitochondrial enzyme activity. Am J Physiol Regul Integr Comp Physiol 2007; 292: R1271-1278.

394. He J, Watkins S, Kelley DE. Skeletal muscle lipid content and oxidative enzyme activity in relation to muscle fiber type in type 2 diabetes and obesity. Diabetes 2001; 50: 817-823.

395. Mensink M, Blaak EE, van Baak MA, Wagenmakers AJ, Saris WH. Plasma free Fatty Acid uptake and oxidation are already diminished in subjects at high risk for developing type 2 diabetes. Diabetes 2001; 50: 2548-2554.

396. Kelley DE, Goodpaster B, Wing RR, Simoneau JA. Skeletal muscle fatty acid metabolism in association with insulin resistance, obesity, and weight loss. Am $J$ Physiol 1999; 277: E1130-1141.

397. Bruce CR, Anderson MJ, Carey AL, et al. Muscle oxidative capacity is a better predictor of insulin sensitivity than lipid status. J Clin Endocrinol Metab 2003; 88: 5444-5451. 
398. Simoneau JA, Veerkamp JH, Turcotte LP, Kelley DE. Markers of capacity to utilize fatty acids in human skeletal muscle: relation to insulin resistance and obesity and effects of weight loss. FASEB J 1999; 13: 2051-2060.

399. Bosma M, Kersten S, Hesselink MK, Schrauwen P. Re-evaluating lipotoxic triggers in skeletal muscle: relating intramyocellular lipid metabolism to insulin sensitivity. Prog Lipid Res 2012; 51: 36-49.

400. Moro C, Bajpeyi S, Smith SR. Determinants of intramyocellular triglyceride turnover: implications for insulin sensitivity. Am J Physiol Endocrinol Metab 2008; 294: E203213.

401. Muoio DM. Intramuscular triacylglycerol and insulin resistance: guilty as charged or wrongly accused? Biochim Biophys Acta 2010; 1801: 281-288.

402. Samuel VT, Shulman GI. Mechanisms for insulin resistance: common threads and missing links. Cell 2012; 148: 852-871.

403. Summers SA. Ceramides in insulin resistance and lipotoxicity. Prog Lipid Res 2006; 45: 42-72.

404. Jocken JW, Goossens GH, Boon H, et al. Insulin-mediated suppression of lipolysis in adipose tissue and skeletal muscle of obese type 2 diabetic men and men with normal glucose tolerance. Diabetologia 2013; 56: 2255-2265.

405. Bergman BC, Hunerdosse DM, Kerege A, Playdon MC, Perreault L. Localisation and composition of skeletal muscle diacylglycerol predicts insulin resistance in humans. Diabetologia 2012; 55: 1140-1150.

406. Nowotny B, Zahiragic L, Krog D, et al. Mechanisms underlying the onset of oral lipidinduced skeletal muscle insulin resistance in humans. Diabetes 2013; 62: 2240-2248.

407. Varma V, Yao-Borengasser A, Rasouli N, et al. Muscle inflammatory response and insulin resistance: synergistic interaction between macrophages and fatty acids leads to impaired insulin action. Am J Physiol Endocrinol Metab 2009; 296: E1300-1310.

408. Bouzakri K, Plomgaard P, Berney T, Donath MY, Pedersen BK, Halban PA. Bimodal effect on pancreatic beta-cells of secretory products from normal or insulin-resistant human skeletal muscle. Diabetes 2011; 60: 1111-1121.

409. Di Gregorio GB, Yao-Borengasser A, Rasouli N, et al. Expression of CD68 and macrophage chemoattractant protein-1 genes in human adipose and muscle tissues: association with cytokine expression, insulin resistance, and reduction by pioglitazone. Diabetes 2005; 54: 2305-2313.

410. Bruun JM, Helge JW, Richelsen B, Stallknecht B. Diet and exercise reduce low-grade inflammation and macrophage infiltration in adipose tissue but not in skeletal muscle in severely obese subjects. Am J Physiol Endocrinol Metab 2006; 290: E961-967.

411. Tam CS, Sparks LM, Johannsen DL, Covington JD, Church TS, Ravussin E. Low macrophage accumulation in skeletal muscle of obese type 2 diabetics and elderly subjects. Obesity (Silver Spring) 2012; 20: 1530-1533.

412. Patsouris D, Cao JJ, Vial G, et al. Insulin resistance is associated with MCP1mediated macrophage accumulation in skeletal muscle in mice and humans. PLoS One 2014; 9: e110653.

413. Deldicque L, Hespel P, Francaux M. Endoplasmic reticulum stress in skeletal muscle: origin and metabolic consequences. Exerc Sport Sci Rev 2012; 40: 43-49.

414. Moors CC, van der Zijl NJ, Diamant M, Blaak EE, Goossens GH. Impaired insulin sensitivity is accompanied by disturbances in skeletal muscle fatty acid handling in subjects with impaired glucose metabolism. Int J Obes (Lond) 2012; 36: 709-717.

415. Wang $\mathrm{H}$, Knaub LA, Jensen DR, et al. Skeletal muscle-specific deletion of lipoprotein lipase enhances insulin signaling in skeletal muscle but causes insulin resistance in liver and other tissues. Diabetes 2009; 58: 116-124.

416. Kim JK, Fillmore JJ, Chen Y, et al. Tissue-specific overexpression of lipoprotein lipase causes tissue-specific insulin resistance. Proc Natl Acad Sci U S A 2001; 98: 7522-7527. 
417. Ladu MJ, Kapsas H, Palmer WK. Regulation of lipoprotein lipase in adipose and muscle tissues during fasting. Am J Physiol 1991; 260: R953-959.

418. Sugden MC, Holness MJ, Howard RM. Changes in lipoprotein lipase activities in adipose tissue, heart and skeletal muscle during continuous or interrupted feeding. Biochem J 1993; 292 ( Pt 1): 113-119.

419. Hamilton MT, Etienne J, McClure WC, Pavey BS, Holloway AK. Role of local contractile activity and muscle fiber type on LPL regulation during exercise. $A m \mathrm{~J}$ Physiol 1998; 275: E1016-1022.

420. Greiwe JS, Holloszy JO, Semenkovich CF. Exercise induces lipoprotein lipase and GLUT-4 protein in muscle independent of adrenergic-receptor signaling. $J$ Appl Physiol (1985) 2000; 89: 176-181.

421. Schrauwen-Hinderling VB, Hesselink MK, Moonen-Kornips E, et al. Short-term training is accompanied by a down regulation of ACC2 mRNA in skeletal muscle. Int J Sports Med 2006; 27: 786-791.

422. Vissing $\mathrm{K}$, Andersen $\mathrm{JL}$, Schjerling $\mathrm{P}$. Are exercise-induced genes induced by exercise? FASEB J 2005; 19: 94-96.

423. Farese RV, Jr., Yost TJ, Eckel RH. Tissue-specific regulation of lipoprotein lipase activity by insulin/glucose in normal-weight humans. Metabolism 1991; 40: 214-216.

424. Dijk W, Kersten S. Regulation of lipoprotein lipase by Angptl4. Trends Endocrinol Metab 2014; 25: 146-155.

425. Xu A, Lam MC, Chan KW, et al. Angiopoietin-like protein 4 decreases blood glucose and improves glucose tolerance but induces hyperlipidemia and hepatic steatosis in mice. Proc Natl Acad Sci U S A 2005; 102: 6086-6091.

426. Votruba SB, Jensen MD. Regional fat deposition as a factor in FFA metabolism. Annu Rev Nutr 2007; 27: 149-163.

427. Abumrad N, Coburn C, Ibrahimi A. Membrane proteins implicated in long-chain fatty acid uptake by mammalian cells: CD36, FATP and FABPm. Biochim Biophys Acta 1999; 1441: 4-13.

428. Glatz JF, Bonen A, Luiken JJ. Exercise and insulin increase muscle fatty acid uptake by recruiting putative fatty acid transporters to the sarcolemma. Curr Opin Clin Nutr Metab Care 2002; 5: 365-370.

429. Wu Q, Ortegon AM, Tsang B, Doege $H$, Feingold KR, Stahl A. FATP1 is an insulinsensitive fatty acid transporter involved in diet-induced obesity. Mol Cell Biol 2006; 26: 3455-3467.

430. Gimeno RE, Ortegon AM, Patel S, et al. Characterization of a heart-specific fatty acid transport protein. J Biol Chem 2003; 278: 16039-16044.

431. Coburn CT, Knapp FF, Jr., Febbraio M, Beets AL, Silverstein RL, Abumrad NA. Defective uptake and utilization of long chain fatty acids in muscle and adipose tissues of CD36 knockout mice. J Biol Chem 2000; 275: 32523-32529.

432. Bonen A, Benton CR, Campbell SE, et al. Plasmalemmal fatty acid transport is regulated in heart and skeletal muscle by contraction, insulin and leptin, and in obesity and diabetes. Acta Physiol Scand 2003; 178: 347-356.

433. Corpeleijn E, Pelsers MM, Soenen S, et al. Insulin acutely upregulates protein expression of the fatty acid transporter CD36 in human skeletal muscle in vivo. $J$ Physiol Pharmacol 2008; 59: 77-83.

434. Bonen A, Parolin ML, Steinberg GR, et al. Triacylglycerol accumulation in human obesity and type 2 diabetes is associated with increased rates of skeletal muscle fatty acid transport and increased sarcolemmal FAT/CD36. FASEB $J$ 2004; 18: 11441146.

435. Corpeleijn E, Mensink M, Kooi ME, Roekaerts PM, Saris WH, Blaak EE. Impaired skeletal muscle substrate oxidation in glucose-intolerant men improves after weight loss. Obesity (Silver Spring) 2008; 16: 1025-1032. 
436. van Hees AM, Jans A, Hul GB, Roche HM, Saris WH, Blaak EE. Skeletal muscle fatty acid handling in insulin resistant men. Obesity (Silver Spring) 2011; 19: 13501359.

437. Jocken JW, Goossens GH, van Hees AM, et al. Effect of beta-adrenergic stimulation on whole-body and abdominal subcutaneous adipose tissue lipolysis in lean and obese men. Diabetologia 2008; 51: 320-327.

438. Glatz JF, Luiken JJ, van Bilsen M, van der Vusse GJ. Cellular lipid binding proteins as facilitators and regulators of lipid metabolism. Mol Cell Biochem 2002; 239: 3-7.

439. Watt MJ. Triglyceride lipases alter fuel metabolism and mitochondrial gene expression. Appl Physiol Nutr Metab 2009; 34: 340-347.

440. Timmers S, de Vogel-van den Bosch J, Hesselink MK, et al. Paradoxical increase in TAG and DAG content parallel the insulin sensitizing effect of unilateral DGAT1 overexpression in rat skeletal muscle. PLoS One 2011; 6: e14503.

441. Amati F, Dube JJ, Alvarez-Carnero E, et al. Skeletal muscle triglycerides, diacylglycerols, and ceramides in insulin resistance: another paradox in endurancetrained athletes? Diabetes 2011; 60: 2588-2597.

442. Dube JJ, Amati F, Toledo FG, et al. Effects of weight loss and exercise on insulin resistance, and intramyocellular triacylglycerol, diacylglycerol and ceramide. Diabetologia 2011; 54: 1147-1156.

443. Pinnamaneni SK, Southgate RJ, Febbraio MA, Watt MJ. Stearoyl CoA desaturase 1 is elevated in obesity but protects against fatty acid-induced skeletal muscle insulin resistance in vitro. Diabetologia 2006; 49: 3027-3037.

444. Hulver MW, Berggren JR, Carper MJ, et al. Elevated stearoyl-CoA desaturase-1 expression in skeletal muscle contributes to abnormal fatty acid partitioning in obese humans. Cell Metab 2005; 2: 251-261.

445. Bergman BC, Perreault L, Hunerdosse DM, Koehler MC, Samek AM, Eckel RH. Intramuscular lipid metabolism in the insulin resistance of smoking. Diabetes 2009; 58: 2220-2227.

446. Perreault L, Bergman BC, Hunerdosse DM, Playdon MC, Eckel RH. Inflexibility in intramuscular triglyceride fractional synthesis distinguishes prediabetes from obesity in humans. Obesity (Silver Spring) 2010; 18: 1524-1531.

447. Moors CC, Blaak EE, van der Zijl NJ, Diamant M, Goossens GH. The effects of longterm valsartan treatment on skeletal muscle fatty acid handling in humans with impaired glucose metabolism. J Clin Endocrinol Metab 2013; 98: E891-896.

448. Sparks LM, Bosma M, Brouwers B, et al. Reduced incorporation of fatty acids into triacylglycerol in myotubes from obese individuals with type 2 diabetes. Diabetes 2014; 63: 1583-1593.

449. Haemmerle G, Lass A, Zimmermann R, et al. Defective lipolysis and altered energy metabolism in mice lacking adipose triglyceride lipase. Science 2006; 312: 734-737.

450. Badin PM, Louche K, Mairal A, et al. Altered skeletal muscle lipase expression and activity contribute to insulin resistance in humans. Diabetes 2011; 60: 1734-1742.

451. Natali A, Gastaldelli A, Camastra S, et al. Metabolic consequences of adipose triglyceride lipase deficiency in humans: an in vivo study in patients with neutral lipid storage disease with myopathy. J Clin Endocrinol Metab 2013; 98: E1540-1548.

452. Blaak EE, Schiffelers SL, Saris WH, Mensink M, Kooi ME. Impaired betaadrenergically mediated lipolysis in skeletal muscle of obese subjects. Diabetologia 2004; 47: 1462-1468.

453. Jocken JW, Roepstorff C, Goossens GH, et al. Hormone-sensitive lipase serine phosphorylation and glycerol exchange across skeletal muscle in lean and obese subjects: effect of beta-adrenergic stimulation. Diabetes 2008; 57: 1834-1841.

454. Gjelstad IM, Haugen F, Gulseth HL, et al. Expression of perilipins in human skeletal muscle in vitro and in vivo in relation to diet, exercise and energy balance. Arch Physiol Biochem 2012; 118: 22-30. 
455. Bosma M, Hesselink MK, Sparks LM, et al. Perilipin 2 improves insulin sensitivity in skeletal muscle despite elevated intramuscular lipid levels. Diabetes 2012; 61: 26792690.

456. Bosma M, Sparks LM, Hooiveld GJ, et al. Overexpression of PLIN5 in skeletal muscle promotes oxidative gene expression and intramyocellular lipid content without compromising insulin sensitivity. Biochim Biophys Acta 2013; 1831: 844-852.

457. Minnaard R, Schrauwen P, Schaart G, et al. Adipocyte differentiation-related protein and OXPAT in rat and human skeletal muscle: involvement in lipid accumulation and type 2 diabetes mellitus. J Clin Endocrinol Metab 2009; 94: 4077-4085.

458. MacPherson RE, Ramos SV, Vandenboom R, Roy BD, Peters SJ. Skeletal muscle PLIN proteins, ATGL and CGI-58, interactions at rest and following stimulated contraction. Am J Physiol Regul Integr Comp Physiol 2013; 304: R644-650.

459. Corpeleijn E, Saris WH, Blaak EE. Metabolic flexibility in the development of insulin resistance and type 2 diabetes: effects of lifestyle. Obes Rev 2009; 10: 178-193.

460. Kelley DE, Simoneau JA. Impaired free fatty acid utilization by skeletal muscle in non-insulin-dependent diabetes mellitus. J Clin Invest 1994; 94: 2349-2356.

461. Blaak EE, Wagenmakers AJ, Glatz JF, et al. Plasma FFA utilization and fatty acidbinding protein content are diminished in type 2 diabetic muscle. Am J Physiol Endocrinol Metab 2000; 279: E146-154.

462. Colberg SR, Simoneau JA, Thaete FL, Kelley DE. Skeletal muscle utilization of free fatty acids in women with visceral obesity. J Clin Invest 1995; 95: 1846-1853.

463. Blaak EE, van Aggel-Leijssen DP, Wagenmakers AJ, Saris WH, van Baak MA. Impaired oxidation of plasma-derived fatty acids in type 2 diabetic subjects during moderate-intensity exercise. Diabetes 2000; 49: 2102-2107.

464. Blaak EE, Van Baak MA, Kemerink GJ, Pakbiers MT, Heidendal GA, Saris WH. Betaadrenergic stimulation of energy expenditure and forearm skeletal muscle metabolism in lean and obese men. Am J Physiol 1994; 267: E306-315.

465. Corpeleijn E, Hessvik NP, Bakke SS, et al. Oxidation of intramyocellular lipids is dependent on mitochondrial function and the availability of extracellular fatty acids. Am J Physiol Endocrinol Metab 2010; 299: E14-22.

466. van Loon LJ, Manders RJ, Koopman R, et al. Inhibition of adipose tissue lipolysis increases intramuscular lipid use in type 2 diabetic patients. Diabetologia 2005; 48: 2097-2107.

467. Haemmerle G, Moustafa T, Woelkart G, et al. ATGL-mediated fat catabolism regulates cardiac mitochondrial function via PPAR-alpha and PGC-1. Nat Med 2011; 17: 1076-1085.

468. Laforet P, Orngreen M, Preisler N, Andersen G, Vissing J. Blocked muscle fat oxidation during exercise in neutral lipid storage disease. Arch Neurol 2012; 69: 530 533.

469. van de Weijer $T$, Havekes B, Bilet $L$, et al. Effects of bezafibrate treatment in a patient and a carrier with mutations in the PNPLA2 gene, causing neutral lipid storage disease with myopathy. Circ Res 2013; 112: e51-54.

470. Engeli S, Birkenfeld AL, Badin PM, et al. Natriuretic peptides enhance the oxidative capacity of human skeletal muscle. J Clin Invest 2012; 122: 4675-4679.

471. Ruderman NB, Saha AK, Vavvas D, Witters LA. Malonyl-CoA, fuel sensing, and insulin resistance. Am J Physiol 1999; 276: E1-E18.

472. Rasmussen BB, Holmback UC, Volpi E, Morio-Liondore B, Paddon-Jones D, Wolfe RR. Malonyl coenzyme $A$ and the regulation of functional carnitine palmitoyltransferase- 1 activity and fat oxidation in human skeletal muscle. $J$ Clin Invest 2002; 110: 1687-1693.

473. Schrauwen P, van Aggel-Leijssen DP, Hul G, et al. The effect of a 3-month lowintensity endurance training program on fat oxidation and acetyl-CoA carboxylase-2 expression. Diabetes 2002; 51: 2220-2226. 
474. Mensink M, Blaak EE, Vidal H, De Bruin TW, Glatz JF, Saris WH. Lifestyle changes and lipid metabolism gene expression and protein content in skeletal muscle of subjects with impaired glucose tolerance. Diabetologia 2003; 46: 1082-1089.

475. He L, Kim T, Long Q, et al. Carnitine palmitoyltransferase-1b deficiency aggravates pressure overload-induced cardiac hypertrophy caused by lipotoxicity. Circulation 2012; 126: 1705-1716.

476. Bruce CR, Hoy AJ, Turner N, et al. Overexpression of carnitine palmitoyltransferase1 in skeletal muscle is sufficient to enhance fatty acid oxidation and improve high-fat diet-induced insulin resistance. Diabetes 2009; 58: 550-558.

477. Mootha VK, Lindgren CM, Eriksson KF, et al. PGC-1alpha-responsive genes involved in oxidative phosphorylation are coordinately downregulated in human diabetes. Nat Genet 2003; 34: 267-273.

478. Patti ME, Butte AJ, Crunkhorn S, et al. Coordinated reduction of genes of oxidative metabolism in humans with insulin resistance and diabetes: Potential role of PGC1 and NRF1. Proc Natl Acad Sci U S A 2003; 100: 8466-8471.

479. Benton CR, Holloway GP, Han XX, et al. Increased levels of peroxisome proliferatoractivated receptor gamma, coactivator 1 alpha (PGC-1alpha) improve lipid utilisation, insulin signalling and glucose transport in skeletal muscle of lean and insulin-resistant obese Zucker rats. Diabetologia 2010; 53: 2008-2019.

480. Phielix E, Schrauwen-Hinderling VB, Mensink $M$, et al. Lower intrinsic ADPstimulated mitochondrial respiration underlies in vivo mitochondrial dysfunction in muscle of male type 2 diabetic patients. Diabetes 2008; 57: 2943-2949.

481. Lowell BB, Shulman GI. Mitochondrial dysfunction and type 2 diabetes. Science 2005; 307: 384-387.

482. Boushel R, Gnaiger E, Schjerling P, Skovbro M, Kraunsoe R, Dela F. Patients with type 2 diabetes have normal mitochondrial function in skeletal muscle. Diabetologia 2007; 50: 790-796.

483. van Tienen FH, Praet SF, de Feyter HM, et al. Physical activity is the key determinant of skeletal muscle mitochondrial function in type 2 diabetes. J Clin Endocrinol Metab 2012; 97: 3261-3269.

484. Gaster M. Reduced TCA flux in diabetic myotubes: A governing influence on the diabetic phenotype? Biochem Biophys Res Commun 2009; 387: 651-655.

485. Ortenblad N, Mogensen M, Petersen I, et al. Reduced insulin-mediated citrate synthase activity in cultured skeletal muscle cells from patients with type 2 diabetes: evidence for an intrinsic oxidative enzyme defect. Biochim Biophys Acta 2005; 1741: 206-214.

486. Petersen KF, Dufour S, Befroy D, Garcia R, Shulman GI. Impaired mitochondrial activity in the insulin-resistant offspring of patients with type 2 diabetes. $N$ Engl J Med 2004; 350: 664-671.

487. Befroy DE, Petersen KF, Dufour S, et al. Impaired mitochondrial substrate oxidation in muscle of insulin-resistant offspring of type 2 diabetic patients. Diabetes 2007; 56 : 1376-1381.

488. Schooneman MG, Vaz FM, Houten SM, Soeters MR. Acylcarnitines: reflecting or inflicting insulin resistance? Diabetes 2013; 62: 1-8.

489. Mercader J, Palou A, Bonet ML. Resveratrol enhances fatty acid oxidation capacity and reduces resistin and Retinol-Binding Protein 4 expression in white adipocytes. $J$ Nutr Biochem 2011; 22: 828-834.

490. Sun $C$, Zhang $F$, Ge X, et al. SIRT1 improves insulin sensitivity under insulinresistant conditions by repressing PTP1B. Cell Metab 2007; 6: 307-319.

491. Lagouge M, Argmann C, Gerhart-Hines Z, et al. Resveratrol improves mitochondrial function and protects against metabolic disease by activating SIRT1 and PGC1alpha. Cell 2006; 127: 1109-1122. 
492. Timmers S, Konings E, Bilet L, et al. Calorie restriction-like effects of 30 days of resveratrol supplementation on energy metabolism and metabolic profile in obese humans. Cell Metab 2011; 14: 612-622.

493. Liu K, Zhou R, Wang B, Mi MT. Effect of resveratrol on glucose control and insulin sensitivity: a meta-analysis of 11 randomized controlled trials. Am J Clin Nutr 2014; 99: 1510-1519.

494. Timmers S, Hesselink MK, Schrauwen P. Therapeutic potential of resveratrol in obesity and type 2 diabetes: new avenues for health benefits? Ann N Y Acad Sci 2013; 1290: 83-89.

495. Roche HM. Fatty acids and the metabolic syndrome. Proc Nutr Soc 2005; 64: 23-29.

496. Jans A, Konings E, Goossens GH, et al. PUFAs acutely affect triacylglycerol-derived skeletal muscle fatty acid uptake and increase postprandial insulin sensitivity. Am J Clin Nutr 2012; 95: 825-836.

497. Bakke SS, Moro C, Nikolic N, et al. Palmitic acid follows a different metabolic pathway than oleic acid in human skeletal muscle cells; lower lipolysis rate despite an increased level of adipose triglyceride lipase. Biochim Biophys Acta 2012; 1821: 1323-1333.

498. Montell E, Turini M, Marotta M, et al. DAG accumulation from saturated fatty acids desensitizes insulin stimulation of glucose uptake in muscle cells. Am J Physiol Endocrinol Metab 2001; 280: E229-237.

499. Gaster M, Rustan AC, Beck-Nielsen H. Differential utilization of saturated palmitate and unsaturated oleate: evidence from cultured myotubes. Diabetes 2005; 54: 648656.

500. Shimabukuro M, Zhou YT, Levi M, Unger RH. Fatty acid-induced beta cell apoptosis: a link between obesity and diabetes. Proc Natl Acad Sci U S A 1998; 95: 2498-2502.

501. Robertson RP, Harmon J, Tran PO, Poitout V. Beta-cell glucose toxicity, lipotoxicity, and chronic oxidative stress in type 2 diabetes. Diabetes 2004; 53 Suppl 1: S119124.

502. Prentki M, Nolan CJ. Islet beta cell failure in type 2 diabetes. J Clin Invest 2006; 116 : 1802-1812.

503. Newsholme P, Keane D, Welters HJ, Morgan NG. Life and death decisions of the pancreatic beta-cell: the role of fatty acids. Clin Sci (Lond) 2007; 112: 27-42.

504. Bollheimer LC, Skelly RH, Chester MW, McGarry JD, Rhodes CJ. Chronic exposure to free fatty acid reduces pancreatic beta cell insulin content by increasing basal insulin secretion that is not compensated for by a corresponding increase in proinsulin biosynthesis translation. J Clin Invest 1998; 101: 1094-1101.

505. Zhou YP, Grill V. Long term exposure to fatty acids and ketones inhibits B-cell functions in human pancreatic islets of Langerhans. J Clin Endocrinol Metab 1995; 80: 1584-1590.

506. Weyer C, Bogardus C, Mott DM, Pratley RE. The natural history of insulin secretory dysfunction and insulin resistance in the pathogenesis of type 2 diabetes mellitus. $J$ Clin Invest 1999; 104: 787-794.

507. Gungor N, Hannon T, Libman I, Bacha F, Arslanian S. Type 2 diabetes mellitus in youth: the complete picture to date. Pediatr Clin North Am 2005; 52: 1579-1609.

508. Poitout V. Beta-cell lipotoxicity: burning fat into heat? Endocrinology 2004; 145: 3563-3565.

509. Sakuraba H, Mizukami H, Yagihashi N, Wada R, Hanyu C, Yagihashi S. Reduced beta-cell mass and expression of oxidative stress-related DNA damage in the islet of Japanese Type II diabetic patients. Diabetologia 2002; 45: 85-96.

510. Butler AE, Janson J, Bonner-Weir S, Ritzel R, Rizza RA, Butler PC. Beta-cell deficit and increased beta-cell apoptosis in humans with type 2 diabetes. Diabetes 2003; 52: $102-110$. 
511. Marchetti P, Del Guerra S, Marselli L, et al. Pancreatic islets from type 2 diabetic patients have functional defects and increased apoptosis that are ameliorated by metformin. J Clin Endocrinol Metab 2004; 89: 5535-5541.

512. Haataja L, Gurlo T, Huang CJ, Butler PC. Islet amyloid in type 2 diabetes, and the toxic oligomer hypothesis. Endocr Rev 2008; 29: 303-316.

513. Giacca A, Xiao C, Oprescu Al, Carpentier AC, Lewis GF. Lipid-induced pancreatic beta-cell dysfunction: focus on in vivo studies. Am J Physiol Endocrinol Metab 2011; 300: E255-262.

514. Meier JJ, Breuer TG, Bonadonna RC, et al. Pancreatic diabetes manifests when beta cell area declines by approximately $65 \%$ in humans. Diabetologia 2012; 55: 13461354.

515. Donath MY, Ehses JA, Maedler K, et al. Mechanisms of beta-cell death in type 2 diabetes. Diabetes 2005; 54 Suppl 2: S108-113.

516. Evans JL, Goldfine ID, Maddux BA, Grodsky GM. Are oxidative stress-activated signaling pathways mediators of insulin resistance and beta-cell dysfunction? Diabetes 2003; 52: 1-8.

517. Green K, Brand MD, Murphy MP. Prevention of mitochondrial oxidative damage as a therapeutic strategy in diabetes. Diabetes 2004; 53 Suppl 1: S110-118.

518. Maedler K, Sergeev P, Ris F, et al. Glucose-induced beta cell production of IL-1beta contributes to glucotoxicity in human pancreatic islets. J Clin Invest 2002; 110: 851860.

519. Donath MY, Storling J, Berchtold LA, Billestrup N, Mandrup-Poulsen T. Cytokines and beta-cell biology: from concept to clinical translation. Endocr Rev 2008; 29: 334350.

520. Scheuner D, Kaufman RJ. The unfolded protein response: a pathway that links insulin demand with beta-cell failure and diabetes. Endocr Rev 2008; 29: 317-333.

521. Back SH, Kaufman RJ. Endoplasmic reticulum stress and type 2 diabetes. Annu Rev Biochem 2012; 81: 767-793.

522. Back SH, Kang SW, Han J, Chung HT. Endoplasmic reticulum stress in the beta-cell pathogenesis of type 2 diabetes. Exp Diabetes Res 2012; 2012: 618396.

523. Evans-Molina C, Hatanaka M, Mirmira RG. Lost in translation: endoplasmic reticulum stress and the decline of beta-cell health in diabetes mellitus. Diabetes Obes Metab 2013; 15 Suppl 3: 159-169.

524. Muoio DM, Newgard CB. Mechanisms of disease: molecular and metabolic mechanisms of insulin resistance and beta-cell failure in type 2 diabetes. Nat Rev Mol Cell Biol 2008; 9: 193-205.

525. Supale S, Li N, Brun T, Maechler P. Mitochondrial dysfunction in pancreatic beta cells. Trends Endocrinol Metab 2012; 23: 477-487.

526. Sharma RB, Alonso LC. Lipotoxicity in the pancreatic Beta cell: not just survival and function, but proliferation as well? Curr Diab Rep 2014; 14: 492.

527. Oh YS, Jun HS. Role of bioactive food components in diabetes prevention: effects on Beta-cell function and preservation. Nutr Metab Insights 2014; 7: 51-59.

528. Nogueira JP, Brites FD. Role of enterocytes in dyslipidemia of insulin-resistant states. Endocrinol Nutr 2013; 60: 179-189.

529. Ding S, Lund PK. Role of intestinal inflammation as an early event in obesity and insulin resistance. Curr Opin Clin Nutr Metab Care 2011; 14: 328-333.

530. Duez H, Lamarche B, Uffelman KD, Valero R, Cohn JS, Lewis GF. Hyperinsulinemia is associated with increased production rate of intestinal apolipoprotein B-48containing lipoproteins in humans. Arterioscler Thromb Vasc Biol 2006; 26: 13571363.

531. Abumrad NA, Davidson NO. Role of the gut in lipid homeostasis. Physiol Rev 2012; 92: 1061-1085. 
532. Iqbal J, Hussain MM. Intestinal lipid absorption. Am J Physiol Endocrinol Metab 2009; 296: E1183-1194.

533. Duee PH, Darcy-Vrillon B, Blachier F, Morel MT. Fuel selection in intestinal cells. Proc Nutr Soc 1995; 54: 83-94.

534. Fleming SE, Fitch MD, DeVries S, Liu ML, Kight C. Nutrient utilization by cells isolated from rat jejunum, cecum and colon. J Nutr 1991; 121: 869-878.

535. Storch J, Zhou YX, Lagakos WS. Metabolism of apical versus basolateral sn-2monoacylglycerol and fatty acids in rodent small intestine. J Lipid Res 2008; 49: 1762-1769.

536. Demignot S, Beilstein F, Morel E. Triglyceride-rich lipoproteins and cytosolic lipid droplets in enterocytes: key players in intestinal physiology and metabolic disorders. Biochimie 2014; 96: 48-55.

537. Zhu J, Lee B, Buhman KK, Cheng JX. A dynamic, cytoplasmic triacylglycerol pool in enterocytes revealed by ex vivo and in vivo coherent anti-Stokes Raman scattering imaging. J Lipid Res 2009; 50: 1080-1089.

538. Bouchoux J, Beilstein F, Pauquai $\mathrm{T}$, et al. The proteome of cytosolic lipid droplets isolated from differentiated Caco-2/TC7 enterocytes reveals cell-specific characteristics. Biol Cell 2011; 103: 499-517.

539. Rivellese AA, De Natale C, Di Marino L, et al. Exogenous and endogenous postprandial lipid abnormalities in type 2 diabetic patients with optimal blood glucose control and optimal fasting triglyceride levels. J Clin Endocrinol Metab 2004; 89: 2153-2159.

540. Kolovou GD, Anagnostopoulou KK, Pavlidis AN, et al. Postprandial lipemia in men with metabolic syndrome, hypertensives and healthy subjects. Lipids Health Dis 2005; 4: 21.

541. Patsch JR, Miesenbock G, Hopferwieser T, et al. Relation of triglyceride metabolism and coronary artery disease. Studies in the postprandial state. Arterioscler Thromb 1992; 12: 1336-1345.

542. Miller M, Stone NJ, Ballantyne C, et al. Triglycerides and cardiovascular disease: a scientific statement from the American Heart Association. Circulation 2011; 123: 2292-2333.

543. Kimura R, Takahashi N, Murota K, et al. Activation of peroxisome proliferatoractivated receptor-alpha (PPARalpha) suppresses postprandial lipidemia through fatty acid oxidation in enterocytes. Biochem Biophys Res Commun 2011; 410: 1-6.

544. Mori T, Kondo H, Hase T, Tokimitsu I, Murase T. Dietary fish oil upregulates intestinal lipid metabolism and reduces body weight gain in C57BL/6J mice. J Nutr 2007; 137: 2629-2634.

545. van Schothorst EM, Flachs P, Franssen-van Hal NL, et al. Induction of lipid oxidation by polyunsaturated fatty acids of marine origin in small intestine of mice fed a high-fat diet. BMC Genomics 2009; 10: 110.

546. Murase T, Aoki M, Wakisaka T, Hase T, Tokimitsu I. Anti-obesity effect of dietary diacylglycerol in C57BL/6J mice: dietary diacylglycerol stimulates intestinal lipid metabolism. J Lipid Res 2002; 43: 1312-1319.

547. Warnakula S, Hsieh J, Adeli K, Hussain MM, Tso P, Proctor SD. New insights into how the intestine can regulate lipid homeostasis and impact vascular disease: frontiers for new pharmaceutical therapies to lower cardiovascular disease risk. Can J Cardiol 2011; 27: 183-191.

548. Xie Y, Newberry EP, Young SG, et al. Compensatory increase in hepatic lipogenesis in mice with conditional intestine-specific Mttp deficiency. J Biol Chem 2006; 281: 4075-4086.

549. Mera Y, Odani N, Kawai T, et al. Pharmacological characterization of diethyl-2-(\{3dimethylcarbamoyl-4-[(4'-trifluoromethylbiphenyl-2-carbonyl)amino]p 
henyl\}acetyloxymethyl)-2-phenylmalonate (JTT-130), an intestine-specific inhibitor of microsomal triglyceride transfer protein. J Pharmacol Exp Ther 2011; 336: 321-327.

550. Sakata S, Ito M, Mera Y, et al. JTT-130, a novel intestine-specific inhibitor of microsomal triglyceride transfer protein, improves hyperglycemia and dyslipidemia independent of suppression of food intake in diabetic rats. J Diabetes Res 2014; 2014: 803832.

551. Hata T, Mera Y, Kawai T, et al. JTT-130, a novel intestine-specific inhibitor of microsomal triglyceride transfer protein, ameliorates impaired glucose and lipid metabolism in Zucker diabetic fatty rats. Diabetes Obes Metab 2011; 13: 629-638.

552. Hata T, Mera Y, Tadaki $\mathrm{H}$, et al. JTT-130, a novel intestine-specific inhibitor of microsomal triglyceride transfer protein, suppresses high fat diet-induced obesity and glucose intolerance in Sprague-Dawley rats. Diabetes Obes Metab 2011; 13: 446454.

553. de La Serre CB, Ellis CL, Lee J, Hartman AL, Rutledge JC, Raybould HE. Propensity to high-fat diet-induced obesity in rats is associated with changes in the gut microbiota and gut inflammation. Am J Physiol Gastrointest Liver Physiol 2010; 299: G440-448.

554. de Wit NJ, Bosch-Vermeulen $\mathrm{H}$, de Groot PJ, et al. The role of the small intestine in the development of dietary fat-induced obesity and insulin resistance in C57BL/6J mice. BMC Med Genomics 2008; 1: 14.

555. Spagnuolo MI, Cicalese MP, Caiazzo MA, et al. Relationship between severe obesity and gut inflammation in children: what's next? Ital J Pediatr 2010; 36: 66.

556. Pendyala S, Natarajan V. Redox regulation of Nox proteins. Respir Physiol Neurobiol 2010; 174: 265-271.

557. Pendyala S, Neff LM, Suarez-Farinas M, Holt PR. Diet-induced weight loss reduces colorectal inflammation: implications for colorectal carcinogenesis. Am J Clin Nutr 2011; 93: 234-242.

558. Luck H, Tsai S, Chung J, et al. Regulation of Obesity-Related Insulin Resistance with Gut Anti-inflammatory Agents. Cell Metab 2015; 21: 527-542.

559. Hodin CM, Verdam FJ, Grootjans J, et al. Reduced Paneth cell antimicrobial protein levels correlate with activation of the unfolded protein response in the gut of obese individuals. J Pathol 2011; 225: 276-284.

560. McGuckin MA, Eri RD, Das I, Lourie R, Florin TH. ER stress and the unfolded protein response in intestinal inflammation. Am J Physiol Gastrointest Liver Physiol 2010; 298: G820-832.

561. Ozcan U, Cao Q, Yilmaz E, et al. Endoplasmic reticulum stress links obesity, insulin action, and type 2 diabetes. Science 2004; 306: 457-461.

562. Eckburg PB, Bik EM, Bernstein CN, et al. Diversity of the human intestinal microbial flora. Science 2005; 308: 1635-1638.

563. Gill SR, Pop M, Deboy RT, et al. Metagenomic analysis of the human distal gut microbiome. Science 2006; 312: 1355-1359.

564. Zhu B, Wang X, Li L. Human gut microbiome: the second genome of human body. Protein Cell 2010; 1: 718-725.

565. Arumugam M, Raes J, Pelletier E, et al. Enterotypes of the human gut microbiome. Nature 2011; 473: 174-180.

566. Zoetendal EG, Rajilic-Stojanovic M, de Vos WM. High-throughput diversity and functionality analysis of the gastrointestinal tract microbiota. Gut 2008; 57: 16051615.

567. Cani PD, Delzenne NM. The role of the gut microbiota in energy metabolism and metabolic disease. Curr Pharm Des 2009; 15: 1546-1558.

568. Musso G, Gambino R, Cassader M. Gut microbiota as a regulator of energy homeostasis and ectopic fat deposition: mechanisms and implications for metabolic disorders. Curr Opin Lipidol 2010; 21: 76-83. 
569. Ley RE, Backhed F, Turnbaugh P, Lozupone CA, Knight RD, Gordon Jl. Obesity alters gut microbial ecology. Proc Natl Acad Sci U S A 2005; 102: 11070-11075.

570. Schwiertz A, Taras D, Schafer K, et al. Microbiota and SCFA in lean and overweight healthy subjects. Obesity (Silver Spring) 2010; 18: 190-195.

571. Karlsson FH, Tremaroli V, Nookaew I, et al. Gut metagenome in European women with normal, impaired and diabetic glucose control. Nature 2013; 498: 99-103.

572. Qin J, Li Y, Cai Z, et al. A metagenome-wide association study of gut microbiota in type 2 diabetes. Nature 2012; 490: 55-60.

573. Carvalho BM, Guadagnini D, Tsukumo DM, et al. Modulation of gut microbiota by antibiotics improves insulin signalling in high-fat fed mice. Diabetologia 2012; 55: 2823-2834.

574. Henao-Mejia J, Elinav E, Jin C, et al. Inflammasome-mediated dysbiosis regulates progression of NAFLD and obesity. Nature 2012; 482: 179-185.

575. Collado MC, Isolauri E, Laitinen K, Salminen S. Distinct composition of gut microbiota during pregnancy in overweight and normal-weight women. Am J Clin Nutr 2008; 88: 894-899.

576. Backhed $\mathrm{F}$, Ding $\mathrm{H}$, Wang $\mathrm{T}$, et al. The gut microbiota as an environmental factor that regulates fat storage. Proc Natl Acad Sci U S A 2004; 101: 15718-15723.

577. Turnbaugh PJ, Ley RE, Mahowald MA, Magrini V, Mardis ER, Gordon JI. An obesityassociated gut microbiome with increased capacity for energy harvest. Nature 2006; 444: 1027-1031.

578. Ferraris RP, Vinnakota RR. Intestinal nutrient transport in genetically obese mice. Am J Clin Nutr 1995; 62: 540-546.

579. Warwick BP, Romsos DR. Energy balance in adrenalectomized ob/ob mice: effects of dietary starch and glucose. Am J Physiol 1988; 255: R141-148.

580. Vrieze A, Van Nood E, Holleman F, et al. Transfer of intestinal microbiota from lean donors increases insulin sensitivity in individuals with metabolic syndrome. Gastroenterology 2012; 143: 913-916 e917.

581. Diamant M, Blaak EE, de Vos WM. Do nutrient-gut-microbiota interactions play a role in human obesity, insulin resistance and type 2 diabetes? Obes Rev 2011; 12: 272281.

582. Akira S, Takeda K. Toll-like receptor signalling. Nat Rev Immunol 2004; 4: 499-511.

583. Cani PD, Amar J, Iglesias MA, et al. Metabolic endotoxemia initiates obesity and insulin resistance. Diabetes 2007; 56: 1761-1772.

584. Mortensen PB, Clausen MR. Short-chain fatty acids in the human colon: relation to gastrointestinal health and disease. Scand J Gastroenterol Suppl 1996; 216: 132148.

585. Cummings $\mathrm{JH}$, Englyst HN, Wiggins HS. The role of carbohydrates in lower gut function. Nutr Rev 1986; 44: 50-54.

586. Cummings $\mathrm{JH}$, Englyst $\mathrm{HN}$. Fermentation in the human large intestine and the available substrates. Am J Clin Nutr 1987; 45: 1243-1255.

587. Cummings JH, Pomare EW, Branch WJ, Naylor CP, Macfarlane GT. Short chain fatty acids in human large intestine, portal, hepatic and venous blood. Gut 1987; 28: 12211227.

588. Wong JM, de Souza R, Kendall CW, Emam A, Jenkins DJ. Colonic health: fermentation and short chain fatty acids. J Clin Gastroenterol 2006; 40: 235-243.

589. Topping DL, Clifton PM. Short-chain fatty acids and human colonic function: roles of resistant starch and nonstarch polysaccharides. Physiol Rev 2001; 81: 1031-1064.

590. Macfarlane GT, Macfarlane S. Fermentation in the human large intestine: its physiologic consequences and the potential contribution of prebiotics. $J$ Clin Gastroenterol 2011; 45 Suppl: S120-127.

591. Musso G, Gambino R, Cassader M. Interactions between gut microbiota and host metabolism predisposing to obesity and diabetes. Annu Rev Med 2011; 62: 361-380. 
592. Scheppach W, Bartram P, Richter A, et al. Effect of short-chain fatty acids on the human colonic mucosa in vitro. JPEN J Parenter Enteral Nutr 1992; 16: 43-48.

593. Zaibi MS, Stocker CJ, O'Dowd J, et al. Roles of GPR41 and GPR43 in leptin secretory responses of murine adipocytes to short chain fatty acids. FEBS Lett 2010; 584: 2381-2386.

594. Xiong $\mathrm{Y}$, Miyamoto N, Shibata $\mathrm{K}$, et al. Short-chain fatty acids stimulate leptin production in adipocytes through the G protein-coupled receptor GPR41. Proc Natl Acad Sci U S A 2004; 101: 1045-1050.

595. Al-Lahham SH, Roelofsen $\mathrm{H}$, Priebe $\mathrm{M}$, et al. Regulation of adipokine production in human adipose tissue by propionic acid. Eur J Clin Invest 2010; 40: 401-407.

596. Vinolo MA, Rodrigues HG, Hatanaka E, Sato FT, Sampaio SC, Curi R. Suppressive effect of short-chain fatty acids on production of proinflammatory mediators by neutrophils. J Nutr Biochem 2011; 22: 849-855.

597. Brown AJ, Goldsworthy SM, Barnes AA, et al. The Orphan G protein-coupled receptors GPR41 and GPR43 are activated by propionate and other short chain carboxylic acids. J Biol Chem 2003; 278: 11312-11319.

598. Ridlon JM, Kang DJ, Hylemon PB. Bile salt biotransformations by human intestinal bacteria. J Lipid Res 2006; 47: 241-259.

599. Stacey M, Webb M. Studies on the antibacterial properties of the bile acids and some compounds derived from cholanic acid. Proc R Soc Med 1947; 134: 523-537.

600. Kurdi P, Kawanishi K, Mizutani K, Yokota A. Mechanism of growth inhibition by free bile acids in lactobacilli and bifidobacteria. J Bacteriol 2006; 188: 1979-1986.

601. David LA, Maurice CF, Carmody RN, et al. Diet rapidly and reproducibly alters the human gut microbiome. Nature 2014; 505: 559-563.

602. Cani PD, Lecourt E, Dewulf EM, et al. Gut microbiota fermentation of prebiotics increases satietogenic and incretin gut peptide production with consequences for appetite sensation and glucose response after a meal. Am J Clin Nutr 2009; 90: 1236-1243.

603. Davis LM, Martinez I, Walter J, Hutkins R. A dose dependent impact of prebiotic galactooligosaccharides on the intestinal microbiota of healthy adults. Int $\mathrm{J}$ Food Microbiol 2010; 144: 285-292.

604. Cani PD, Neyrinck AM, Fava F, et al. Selective increases of bifidobacteria in gut microflora improve high-fat-diet-induced diabetes in mice through a mechanism associated with endotoxaemia. Diabetologia 2007; 50: 2374-2383.

605. Roberfroid M, Gibson GR, Hoyles L, et al. Prebiotic effects: metabolic and health benefits. Br J Nutr 2010; 104 Suppl 2: S1-63.

606. Pouteau E, Nguyen P, Ballevre O, Krempf M. Production rates and metabolism of short-chain fatty acids in the colon and whole body using stable isotopes. Proc Nutr Soc 2003; 62: 87-93.

607. Kellow NJ, Coughlan MT, Reid CM. Metabolic benefits of dietary prebiotics in human subjects: a systematic review of randomised controlled trials. $\mathrm{Br} J$ Nutr 2014; 111 : 1147-1161.

608. Chen $\mathrm{CH}$, Wang $\mathrm{Y}$, Nakatsuji $\mathrm{T}$, et al. An innate bactericidal oleic acid effective against skin infection of methicillin-resistant Staphylococcus aureus: a therapy concordant with evolutionary medicine. J Microbiol Biotechnol 2011; 21: 391-399.

609. Desbois AP, Smith VJ. Antibacterial free fatty acids: activities, mechanisms of action and biotechnological potential. Appl Microbiol Biotechnol 2010; 85: 1629-1642.

610. Alcock J, Franklin ML, Kuzawa CW. Nutrient signaling: evolutionary origins of the immune-modulating effects of dietary fat. Q Rev Biol 2012; 87: 187-223.

611. Fava F, Gitau R, Griffin BA, Gibson GR, Tuohy KM, Lovegrove JA. The type and quantity of dietary fat and carbohydrate alter faecal microbiome and short-chain fatty acid excretion in a metabolic syndrome 'at-risk' population. Int $J$ Obes (Lond) 2013; 37: 216-223. 
612. Simoes CD, Maukonen J, Kaprio J, Rissanen A, Pietilainen KH, Saarela M. Habitual dietary intake is associated with stool microbiota composition in monozygotic twins. $J$ Nutr 2013; 143: 417-423.

613. Santacruz A, Marcos A, Warnberg J, et al. Interplay between weight loss and gut microbiota composition in overweight adolescents. Obesity (Silver Spring) 2009; 17: 1906-1915.

614. Brinkworth GD, Noakes M, Clifton PM, Bird AR. Comparative effects of very lowcarbohydrate, high-fat and high-carbohydrate, low-fat weight-loss diets on bowel habit and faecal short-chain fatty acids and bacterial populations. Br J Nutr 2009; 101: 1493-1502.

615. de Wit N, Derrien M, Bosch-Vermeulen $\mathrm{H}$, et al. Saturated fat stimulates obesity and hepatic steatosis and affects gut microbiota composition by an enhanced overflow of dietary fat to the distal intestine. Am J Physiol Gastrointest Liver Physiol 2012; 303: G589-599.

616. Shen W, Gaskins HR, McIntosh MK. Influence of dietary fat on intestinal microbes, inflammation, barrier function and metabolic outcomes. J Nutr Biochem 2014; 25: 270-280.

617. Sayin SI, Wahlstrom A, Felin J, et al. Gut microbiota regulates bile acid metabolism by reducing the levels of tauro-beta-muricholic acid, a naturally occurring FXR antagonist. Cell Metab 2013; 17: 225-235.

618. Kuo SM, Merhige PM, Hagey LR. The effect of dietary prebiotics and probiotics on body weight, large intestine indices, and fecal bile acid profile in wild type and IL10-/mice. PLoS One 2013; 8: e60270.

619. Kim JH, Bae KH, Choi YK, et al. Fibroblast growth factor 21 analogue LY2405319 lowers blood glucose in streptozotocin-induced insulin-deficient diabetic mice by restoring brown adipose tissue function. Diabetes Obes Metab 2015; 17: 161-169. 
CHAPTER 3 


\section{Improved insulin sensitivity with angiotensin receptor neprilysin inhibition in individuals with obesity and hypertension}

Jordan J., Stinkens R.*, Jax T.*, Engeli S., Blaak E.E., May M., Havekes B., Schindler C., Pal P., Heise T., Goossens G.H., Langenickel T.H.

* Shared authorship

Clinical Pharmacology and Therapeutics, 2017; 101(2):254-263 


\begin{abstract}
Natriuretic peptide (NP) deficiency and sustained renin-angiotensin system activation are associated with impaired oxidative metabolism and predispose to type-2 diabetes. We hypothesized that sacubitril/valsartan (LCZ696), which augments NP through neprilysin inhibition while blocking angiotensin II type-1 $\left(\mathrm{AT}_{1}\right)$-receptors, improves insulin sensitivity, lipid mobilization, and oxidation. After 8 weeks of treatment of obese patients with hypertension, sacubitril/valsartan 400 $\mathrm{mg}$ q.d., but not amlodipine $10 \mathrm{mg}$ q.d., was associated with a significant increase from baseline in the insulin sensitivity index (hyperinsulinemic-euglycemic clamp), and tended to be higher in patients treated with sacubitril/valsartan compared to amlodipine. Abdominal adipose tissue interstitial glycerol concentrations increased with sacubitril/valsartan, but decreased with amlodipine. Whole-body lipolysis and substrate oxidation did not change with either treatment. Results confirm that sacubitril/valsartan treatment leads to a metabolic benefit in the study population and supports the relevance of neprilysin inhibition along with $\mathrm{AT}_{1}$-receptor blockade in the regulation of human glucose and lipid metabolism.
\end{abstract}




\section{INTRODUCTION}

Obesity, type-2 diabetes mellitus (T2DM), and cardiovascular diseases are interrelated. Patients with obesity and T2DM are at increased risk for arterial hypertension and heart failure [1,2], whereas patients with arterial hypertension or heart ,failure frequently exhibit impaired muscular oxidative capacity predisposing them to insulin resistance through accumulation of toxic lipid intermediates [3, 4].

The endopeptidase neprilysin is ubiquitously expressed, including in human adipocytes [5]. Its plasma activity positively correlates with measures of obesity and insulin resistance in humans, implicating neprilysin in the regulation of cardiometabolic crosstalk presumably through cleavage of neprilysin substrates with cardiometabolic effects, such as natriuretic peptides (NPs) and angiotensin II [6]. Indeed, patients with obesity [7] or heart failure exhibit absolute or relative natriuretic peptide deficiency, respectively. Circulating atrial natriuretic peptide levels are inversely related to plasma glucose and insulin concentrations, and low atrial natriuretic peptide levels predict the development of both arterial hypertension and diabetes mellitus in humans [8]. Furthermore, polymorphisms in the genes encoding atrial natriuretic peptide and B-type natriuretic peptide contribute to the variability in the risk for type 2 diabetes after adjustment for age, gender, and body mass index [9, 10]. NP promotes lipid mobilization from adipose tissue [11], increases postprandial lipid oxidation [12], elicits adiponectin release, and enhances muscular oxidative capacity $[13,14]$. In contrast to atrial natriuretic peptide, angiotensin II promotes insulin resistance, and angiotensin II type-1 (AT 1 )receptor blockade modestly improved insulin sensitivity and pancreatic beta-cell function in humans [15].

Sacubitril/valsartan (LCZ696), a novel angiotensin receptor neprilysin inhibitor, was recently approved in the United States, Europe, and many other countries to reduce the risk of cardiovascular death and hospitalizations in patients with chronic heart failure (New York Heart Association classes II-IV) and reduced ejection fraction [16]. Upon oral administration, sacubitril/valsartan provides exposure to sacubitril (AHU377), a prodrug that is rapidly metabolized to the biologically active neprilysin inhibitor, sacubitrilat (LBQ657), and to the $\mathrm{AT}_{1}$-receptor blocker valsartan. This active drug augments neprilysin substrates, such as natriuretic peptides, while inhibiting $\mathrm{AT}_{1}$-receptor mediated responses [17].

We hypothesized that sacubitril/valsartan, through its combined mechanism of action, improves peripheral insulin sensitivity and increases lipid mobilization from subcutaneous abdominal adipose tissue in patients with obesity and arterial hypertension.

We conducted a mechanistic metabolic profiling study to investigate the effects of 8-week treatment with sacubitril/valsartan and amlodipine on peripheral insulin sensitivity, abdominal subcutaneous adipose tissue lipolysis, whole-body lipolysis, and energy expenditure and substrate oxidation in patients with obesity and elevated blood pressure. In this study, the patient population served as an exemplary human model for relative natriuretic peptide deficiency and impaired insulin sensitivity, and was specifically selected to support the comparison of sacubitril/valsartan with a metabolically neutral comparator, amlodipine. 


\section{RESULTS}

\section{Subjects}

Of the 98 patients enrolled, (sacubitril/valsartan=50; amlodipine=48), 92 patients $(93.9 \%)$ completed the study. All patients were included in the safety analysis set (Figure 1). Patients with missing baseline or post-treatment assessments were excluded from the pharmacodynamic analysis set, resulting in a maximum of 96 patients (sacubitril/valsartan $=50$; amlodipine $=46$ ) included in the pharmacodynamic analysis set.

Patient demographics and baseline characteristics are summarized in Table 1 and were comparable between the groups.

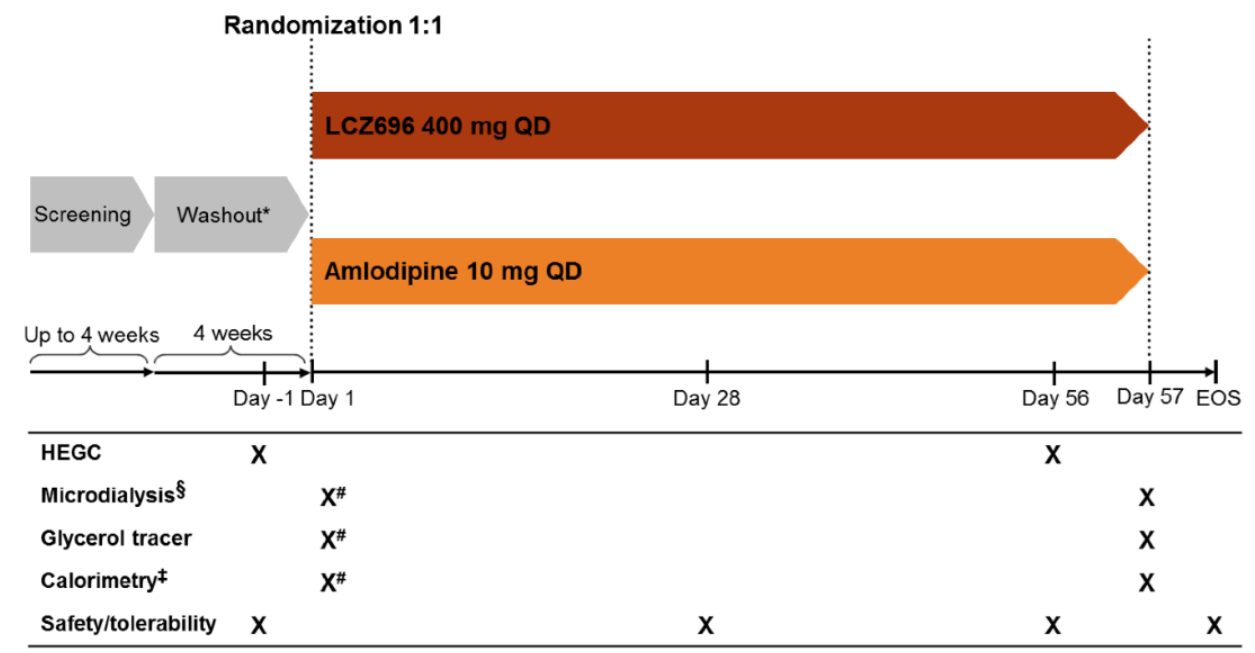

Figure 1. Study design

*Indicates those who had to discontinue antihypertensive medication. \# Indicates those who had to discontinue before the first dose. $\S$ Indicates subcutaneous adipose tissue microdialysis.

$\ddagger$ Indicates indirect calorimetry by Spirometry. EOS, end of study; HEGC, hyperinsulinemic-euglycemic glucose clamp. 


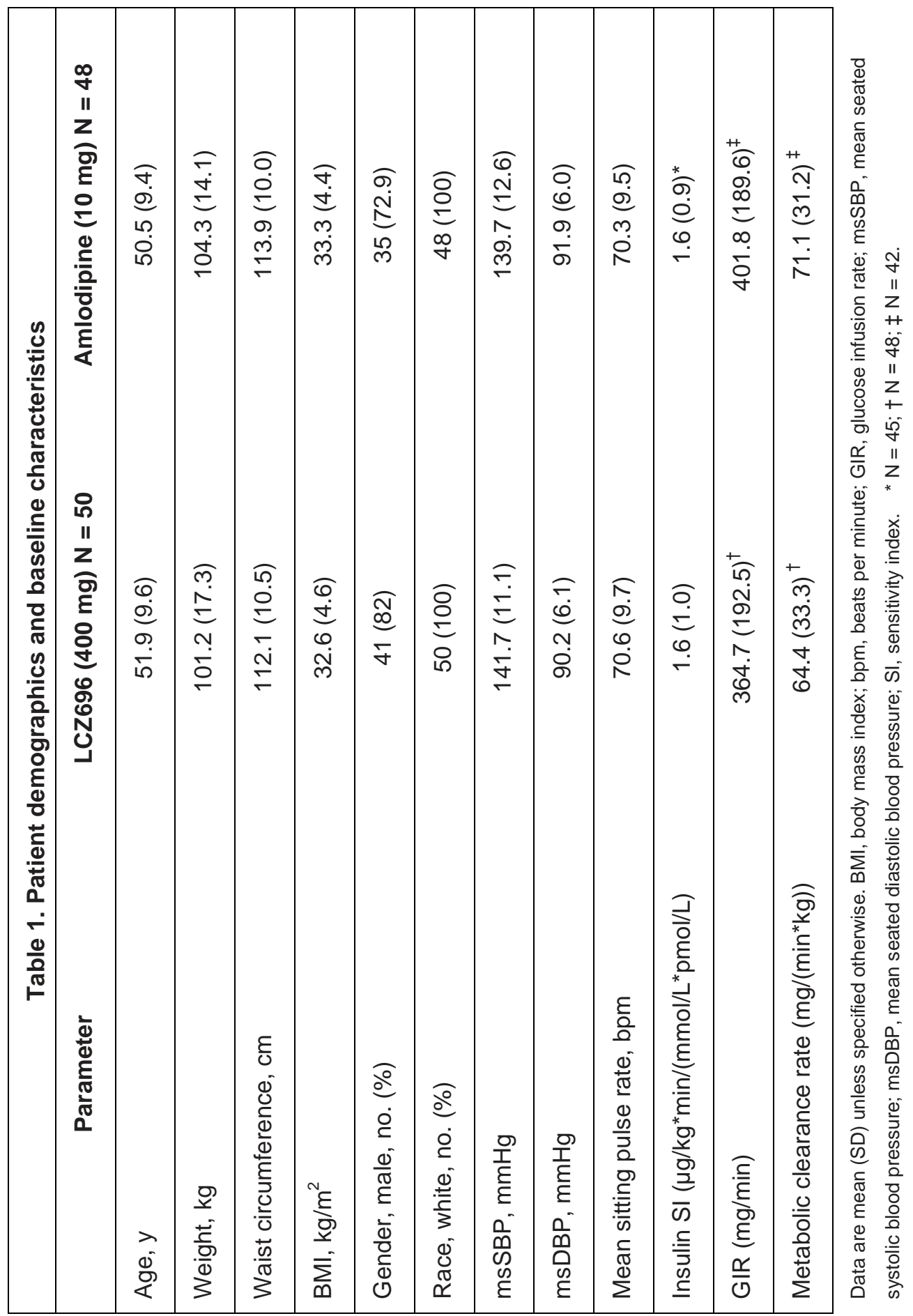




\section{Peripheral insulin sensitivity}

Sacubitril/valsartan, but not amlodipine, was associated with a significant increase from baseline in mean insulin sensitivity index ( $\mathrm{SI})$, as evidenced by the $95 \%$ confidence interval $(\mathrm{Cl}$; mean change from baseline $(95 \% \mathrm{Cl})$; sacubitril/valsartan $0.192(0.025-0.359) \mu \mathrm{g} / \mathrm{kg}^{*} \mathrm{~min} /\left(\mathrm{mmol} / \mathrm{L}^{*} \mathrm{pmol} / \mathrm{L}\right)$; and amlodipine $0.065(-0.116$ to $0.246) \mu \mathrm{g} / \mathrm{kg}^{*} \mathrm{~min} /\left(\mathrm{mmol} / \mathrm{L}^{*} \mathrm{pmol} / \mathrm{L}\right)$; Figure $\left.2 \mathrm{a}\right)$. At week 8 , the $\mathrm{SI}$ trended to be higher after treatment with sacubitril/valsartan vs. amlodipine (sacubitril/valsartan $1.87 \mu \mathrm{g} / \mathrm{kg}^{*} \mathrm{~min} /\left(\mathrm{mmol} / \mathrm{L}^{*} \mathrm{pmol} / \mathrm{L}\right)$; amlodipine $1.76 \mu \mathrm{g} / \mathrm{kg}^{*} \mathrm{~min} /\left(\mathrm{mmol} / \mathrm{L}^{*} \mathrm{pmol} / \mathrm{L}\right)$ ); however, the treatment difference did not reach statistical significance as evidenced by the $97.5 \% \mathrm{Cl}$ (estimated treatment difference $(97.5 \% \mathrm{Cl}), 0.128$ (0.155 to 0.410$\left.) \mu \mathrm{g} / \mathrm{kg}^{*} \mathrm{~min} /\left(\mathrm{mmol} / \mathrm{L}^{*} \mathrm{pmol} / \mathrm{L}\right)\right)$. Importantly, 8 weeks of treatment with sacubitril/valsartan, but not amlodipine, showed a significant increase from baseline and a significant treatment difference in favor of sacubitril/valsartan in glucose infusion rate (GIR; adjusted mean $(95 \% \mathrm{Cl}$ ) at week 8: LCZ696 445.3 (412.3-478.3) mg/min; amlodipine 390.5 (355.2-425.8) $\mathrm{mg} / \mathrm{min}$; estimated change from baseline: sacubitril/valsartan 58.4 (25.4-91.4) $\mathrm{mg} / \mathrm{min}$; and amlodipine 3.6 (31.7 to 38.9$) \mathrm{mg} / \mathrm{min}$; adjusted treatment difference $54.8(6.4-103.3) \mathrm{mg} / \mathrm{min}$, $\mathrm{p}=0.027$; Figure $2 \mathrm{~b})$, the GIR normalized by body weight (adjusted mean $(95 \% \mathrm{Cl})$ at week 8: sacubitril/valsartan 4.49 (4.15-4.83) $\mathrm{mg} /\left(\mathrm{min}^{\star} \mathrm{kg}\right)$; amlodipine 3.88 (3.51-4.24) $\mathrm{mg} /\left(\mathrm{min}^{*} \mathrm{~kg}\right)$; estimated change from baseline: sacubitril/valsartan 0.64 (0.31-0.98) $\mathrm{mg} /\left(\mathrm{min}^{*} \mathrm{~kg}\right)$; amlodipine 0.03 (-0.33 to 0.39$) \mathrm{mg} /\left(\mathrm{min}^{*} \mathrm{~kg}\right)$; adjusted treatment difference $0.61(0.12-1.11) \mathrm{mg} /\left(\mathrm{min}^{*} \mathrm{~kg}\right), \mathrm{p}=0.016$; Figure $\left.2 \mathrm{c}\right)$, and metabolic clearance rate of glucose (adjusted mean $(95 \% \mathrm{Cl})$ at week 8: sacubitril/valsartan 79.1 (73.1-85.0) $\left(\mathrm{L}^{*} \mathrm{mg}\right) /\left(\mathrm{min}^{*} \mathrm{mmol}\right)$; amlodipine 69.4 (63.075.8) $\left(\mathrm{L}^{*} \mathrm{mg}\right) /\left(\mathrm{min}^{*} \mathrm{mmol}\right)$; estimated change from baseline: sacubitril/valsartan 10.7 (4.8-16.7) $\left(\mathrm{L}^{*} \mathrm{mg}\right) /\left(\mathrm{min}^{\star} \mathrm{mmol}\right)$; amlodipine 1.1 (-5.3 to 7.5) $\left(\mathrm{L}^{*} \mathrm{mg}\right) /\left(\mathrm{min}^{\star} \mathrm{mmol}\right)$; and adjusted treatment difference $9.7(0.9-18.4)\left(\mathrm{L}^{*} \mathrm{mg}\right) /\left(\mathrm{min}^{*} \mathrm{mmol}\right), \mathrm{p}=0.031$; Figure 2d).

To assess any significant effect of body weight and waist circumference, the treatment difference for GIR was obtained from a similar analysis of covariance model with additional covariates: change in body weight at week 8 and waist circumference at screening. There was no significant covariate effect in either case (body weight, estimated treatment difference (95\% Cl): 57.57 (8.98-106.15), $\mathrm{p}=0.0208$; waist circumference 50.86 (2.28-99.44), $\mathrm{p}=0.0404)$ compared to the actual treatment difference for GIR (54.85 (6.39-103.30), $p=0.0270)$. Scatter plot of GIR vs. body weight and change in GIR vs. change in body weight did not display any apparent trend of change at day 56 from baseline (Supplementary Figure S1a and $\mathrm{S} 1 \mathrm{~b}$, respectively).

Plasma insulin had reached steady-state values over the last 30 minutes of the clamp procedure (Figure 3). 
A) Insulin Sensitivity Index

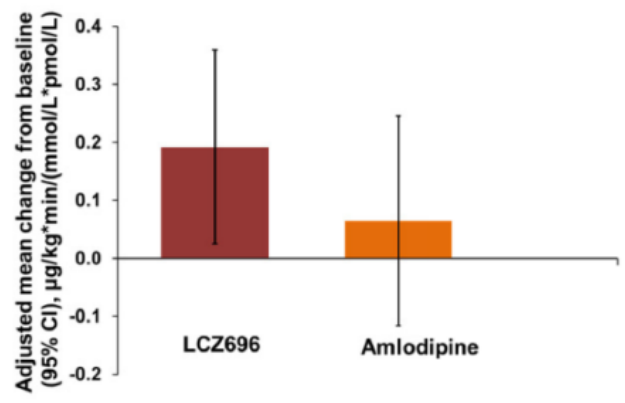

C) Glucose Infusion Rate by Body Weight

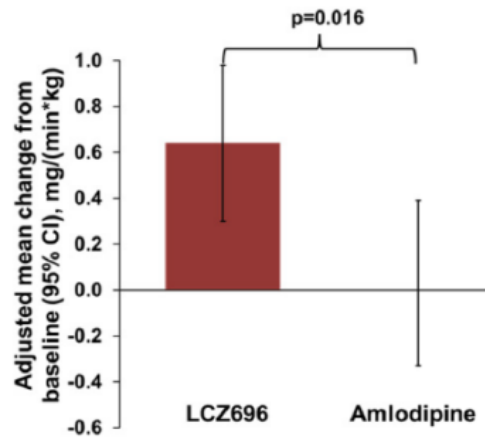

B) Glucose Infusion Rate

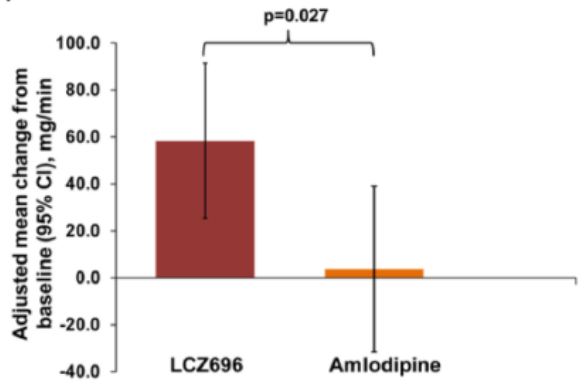

D) Metabolic Clearance Rate

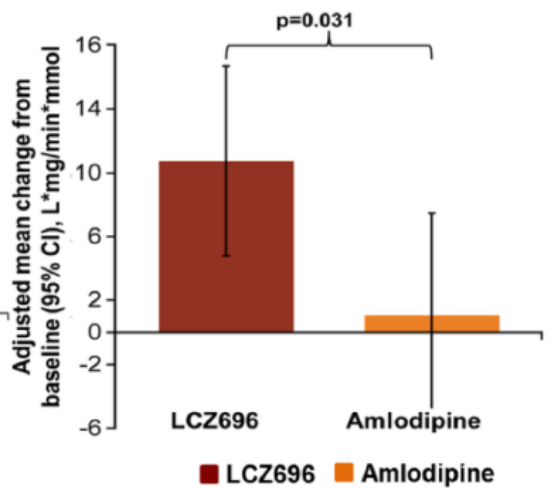

Figure 2. Mean changes from baseline in insulin sensitivity variables after 8-week treatment with sacubitril/valsartan vs. amlodipine.

Insulin sensitivity index (A); glucose infusion rate (GIR) (B); GIR by body weight (C); and metabolic clearance rate (D). $\mathrm{Cl}$, confidence interval.

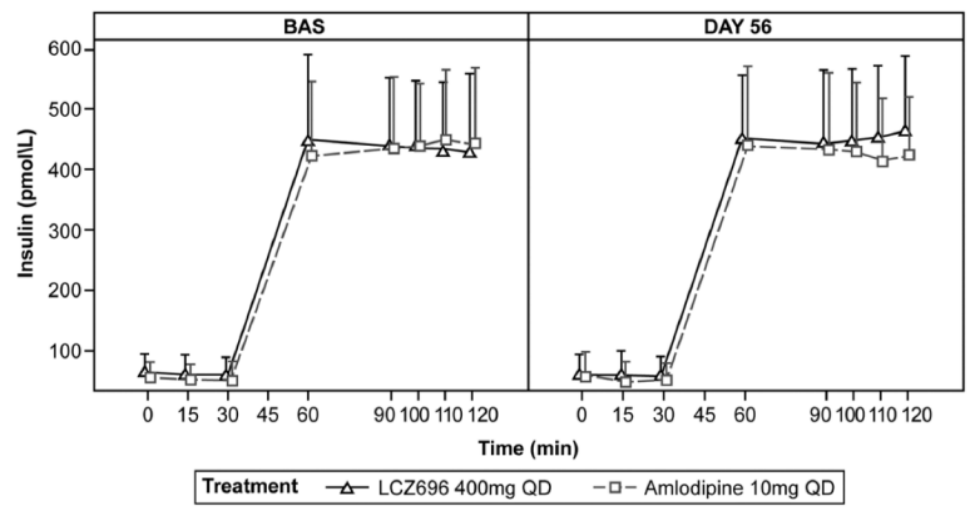

Figure 3. Insulin profiles during the hyperinsulinemic-euglycemic glucose clamp. BAS, baseline. 


\section{Abdominal subcutaneous adipose tissue lipolysis}

The change in ethanol ratio from baseline was significant in the sacubitril/valsartan group at the 45 -minute $(p=0.027)$ and $30+45$-minute $(p=0.042)$ time points. However, there was no significant difference between treatment groups at any of the time points (Figure 4a). An increase in interstitial glycerol from baseline to week 8 was observed at all time points in the sacubitril/valsartan group (adjusted geometric mean ratio to baseline $(95 \% \mathrm{Cl})$ at $30+45$-minute time points 1.05 (0.93-1.18)), but the change did not reach statistical significance. Compared with the amlodipine group, the change from baseline in glycerol levels was significantly higher with sacubitril/valsartan (adjusted geometric mean ratio between treatments $(95 \% \mathrm{Cl})$ at $30+45$-minute time points $1.22(1.03-1.45), \mathrm{p}=0.026$; Figure $4 \mathrm{~b})$. Glucose and lactate levels did not differ significantly from baseline in any of the treatment groups and there was no difference between the treatment groups after 8 weeks of treatment (Figure $4 c$ and $4 d$ ).
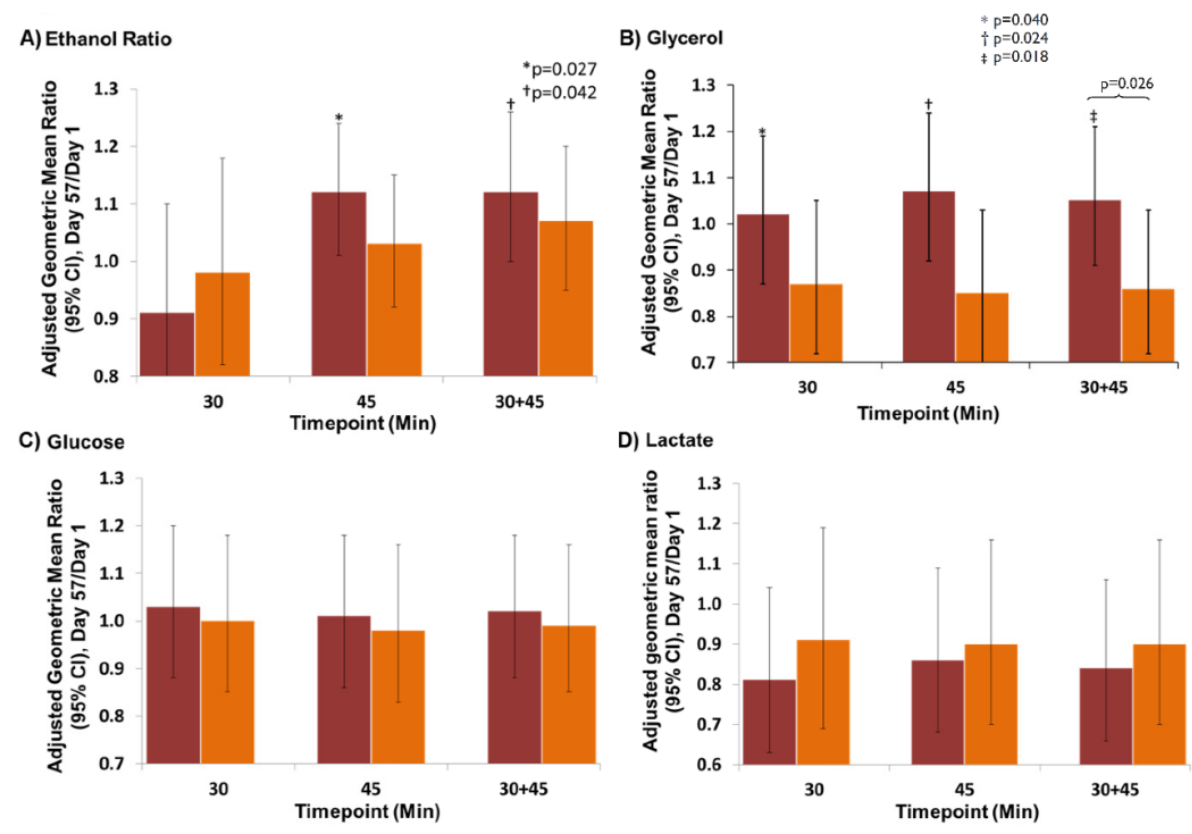

LCZ696 Amlodipine

Figure 4. Adjusted geometric mean ratios of local adipose tissue lipolysis variables after 8-week treatment with sacubitril/valsartan and amlodipine.

Ethanol ratio (A); glycerol (B); glucose (C); and lactate (D). Data were analyzed for repeated measurements on log transformed values with treatment, visit, time and treatment*visit*time interaction as fixed effect assuming an unstructured covariance. Subjects with missing data were excluded from the analysis. The number of missing values ranged from 3 to 6 and 3 to 7 across all variables in the sacubitril/valsartan and amlodipine groups, respectively. $\mathrm{Cl}$, confidence interval. 


\section{Whole-body lipolysis}

The rate of appearance $(95 \% \mathrm{Cl})$ of endogenous plasma glycerol at baseline on day 1 was $172.8(158.0-189.0)$ and $200.6(182.4-220.6) \mu \mathrm{mol} / \mathrm{kg} \cdot \mathrm{min}^{-1}$ in the sacubitril/valsartan and amlodipine groups, respectively. Whole-body glycerol rate of appearance was not significantly affected by amlodipine treatment, but was significantly lower at week 8 compared to baseline in the sacubitril/valsartan group at the $30+45$-minute time point (geometric mean ratio $(95 \% \mathrm{Cl}), 0.93(0.87-1.00)$, $\mathrm{p}=0.045$; Figure 5). There was no change from baseline in the sacubitril/valsartan group at the 45-minute time point. There were no differences between treatment groups in whole-body lipolysis after 8 weeks of treatment.

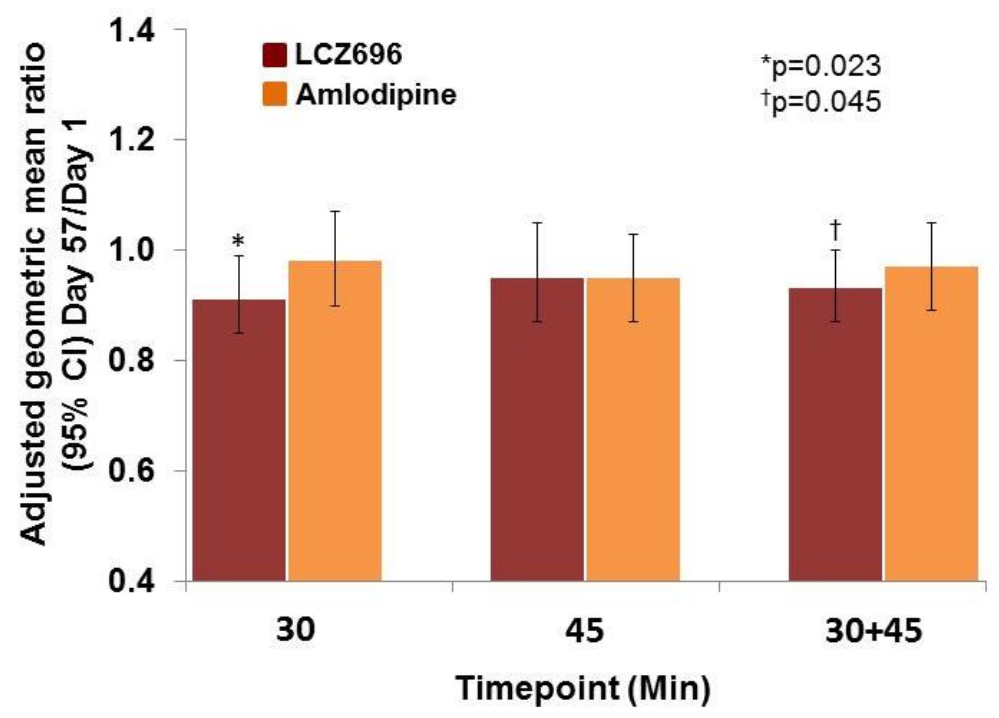

Figure 5. Whole-body lipolysis (rate of glycerol appearance).

The $\mathrm{p}$ value compared with baseline. $\mathrm{Cl}$, confidence interval.

\section{Energy expenditure and substrate oxidation}

At baseline, the mean respiratory quotient and resting energy expenditure were comparable between groups (mean (SD); respiratory quotient: LCZ696 0.773 (0.063), amlodipine $0.768(0.051)$; resting energy expenditure: sacubitril/valsartan 2,134.81 (397.98) kcal; and amlodipine 2,180.30 (548.78) kcal). At week 8, respiratory quotient and resting energy expenditure were not significantly different from baseline in either of the treatment groups or between the treatment groups (mean treatment difference at week $8(95 \% \mathrm{Cl})$; respiratory quotient, $0.012(-0.028$ to 0.051$), \mathrm{p}=0.557$; resting energy expenditure, 231.26 (-149.75 to 87.24$) \mathrm{kcal}$, $\mathrm{p}=0.601)$. 
Circulating metabolites, insulin and catecholamines in the fasted state

At week 8, there were no significant differences from baseline in either treatment group or between treatment groups in fasting plasma glucose, insulin, glycerol, or serum free fatty acids (Supplementary Table S1). However, sacubitril/valsartan, but not amlodipine, was associated with a significant decrease from baseline in plasma epinephrine (ratio to baseline $(95 \% \mathrm{Cl})$ for sacubitril/valsartan, $0.81(0.72-0.91)$, $\mathrm{p}<0.001$ ), resulting in a significant treatment difference (sacubitril/valsartan vs. amlodipine treatment ratio $(95 \% \mathrm{Cl}), 0.77(0.66-0.91), \mathrm{p}=0.003)$. Conversely, amlodipine was associated with a significant increase from baseline in norepinephrine (ratio to baseline $(95 \% \mathrm{Cl})$ for amlodipine, 1.20 (1.07-1.33), $\mathrm{p}=0.001)$; however, the difference between treatments at week 8 was not statistically significant.

\section{Blood pressure}

Baseline data for mean seated blood pressure is presented in Table 1. At week 8, mean seated blood pressure had decreased more in the sacubitril/valsartan group compared with the amlodipine group (systolic blood pressure: $-21.0 \pm 16.1 \mathrm{mmHg}$ vs. $-12.4 \pm 14.7 \mathrm{mmHg}$; diastolic blood pressure: $-12.4 \pm 9.1 \mathrm{mmHg}$ vs. $-10.0 \pm 7.8$ $\mathrm{mmHg}$; Figure 6).

\section{Safety Assessments}

Of the 98 patients, 67 patients (68.4\%) experienced at least one adverse effect $(A E)$. Most AEs were mild or moderate in intensity. The overall AE incidence was lower in the sacubitril/valsartan compared with the amlodipine group $(60.0 \%$ vs. 77.1\%; Supplementary Table S2). Five patients discontinued due to AEs. Two patients discontinued due to a serious adverse event, which was unrelated to the study drug (ruptured cerebral aneurysm in one patient in the sacubitril/valsartan group and nephrolithiasis in one patient in the amlodipine group). Three patients discontinued due to AEs (pruritus in one patient in the sacubitril/valsartan group, and a single case of hypertension and peripheral edema in the amlodipine group). The most commonly occurring AEs were nasopharyngitis, peripheral edema, and headache, each of which had an overall incidence of $>10 \%$ (Supplementary Table S2). AEs suspected to be related to the study drug by the investigators occurred more frequently in the amlodipine group than in patients receiving sacubitril/valsartan ( $46 \%$ vs. $24 \%$ ). Peripheral edema was more common in the amlodipine group, whereas pruritus occurred only in the sacubitril/valsartan group (Supplementary Table S2). Two patients in the sacubitril/valsartan group reported mild orthostatic hypertension, both of which resolved by the end of the study. Both of these events were suspected to be related to the study drug. No deaths were reported in the study. 
(A)

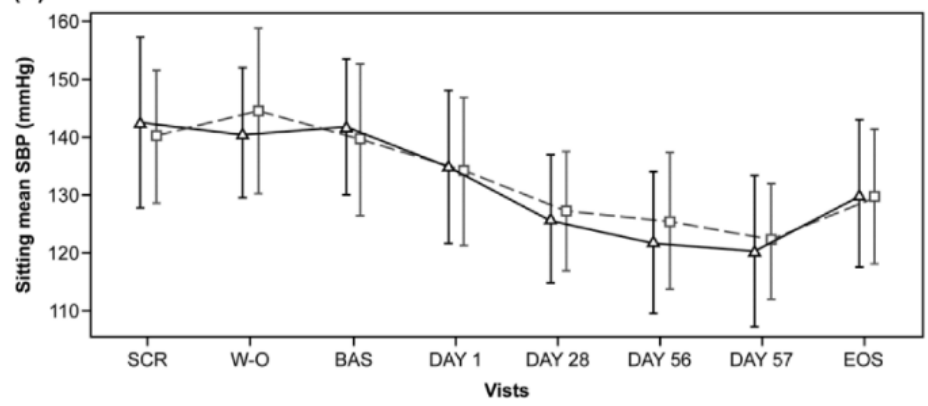

(B)

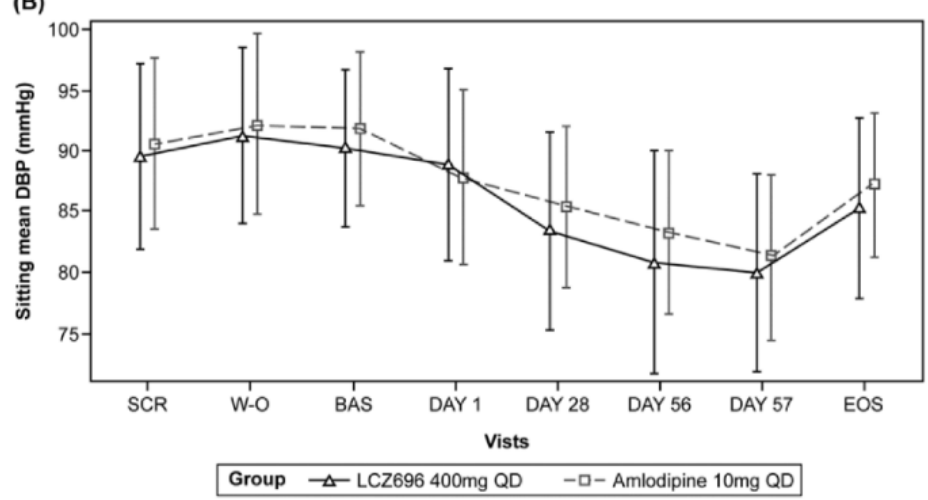

Figure 6. Arithmetic mean (SD) of mean seated systolic blood pressure (SBP) (A); and mean seated diastolic blood pressure (DBP) (B) after 8-week treatment with sacubitril/valsartan and amlodipine.

BAS, baseline; EOS, end of study; SCR, screening; W-O, washout. 


\section{DISCUSSION}

Treatment with sacubitril/valsartan compared with amlodipine for 8 weeks improved peripheral insulin sensitivity in obese patients with elevated blood pressure. This improvement was not explained by changes in body weight or waist circumference. Furthermore, sacubitril/valsartan significantly increased abdominal subcutaneous adipose tissue lipolysis without changing whole-body lipolysis and plasma free fatty acid concentrations. Therefore, this study is the first to show that simultaneous neprilysin inhibition and $\mathrm{AT}_{1}$-receptor blockade regulates glucose and lipid metabolism in humans, supporting the concept that neprilysin substrates and $\mathrm{AT}_{1}$ receptor blockade have an important role in the crosstalk between the cardiovascular system and metabolism in humans. These findings imply that sacubitril/valsartan may improve cardiovascular and metabolic health in patients with cardiovascular disease.

The metabolic effects of sacubitril/valsartan observed here could be mediated through increased availability of neprilysin substrates, $\mathrm{AT}_{1}$-receptor inhibition, or both mechanisms combined. Indeed, individuals with obesity exhibit increased renin angiotensin system (RAS) activity $[18,19]$ both systemically and at the tissue level. Although genetically modified animals overexpressing RAS components are characterized by insulin resistance that improved with pharmacological RAS inhibition [20], the improvement in insulin resistance after RAS inhibition in humans has been less consistent [16]. Among patients with impaired glucose tolerance and cardiovascular disease or risk factors, the use of valsartan for 5 years, along with lifestyle modification, led to a $14 \%$ relative reduction in T2DM incidence [21]. However, ramipril compared with placebo did not reduce the risk of new onset T2DM in patients with impaired fasting glucose levels [22]. Overall, the improvement of glucose metabolism after RAS inhibition seems to be modest and may not solely explain the improvement in insulin sensitivity observed with sacubitril/valsartan treatment. Moreover, $\mathrm{AT}_{1}$-receptor blockade does not increase abdominal subcutaneous adipose tissue lipolysis, which was observed with sacubitril/valsartan treatment $[23,24]$.

The present study suggests that neprilysin inhibition contributed to the metabolic effect of sacubitril/valsartan treatment. Neprilysin degrades multiple peptides that have the potential to modulate lipid and glucose metabolism, such as natriuretic peptides, bradykinin, endothelin-1, and glucagon-like peptide 1. Therefore, we cannot distinguish the contribution of individual neprilysin substrates to the observed metabolic response. Eventually, the pharmacological effect of neprilysin inhibition will depend on the net effect on all biologically relevant neprilysin substrates. For example, bradykinin has been suggested to improve insulin sensitivity and attenuate lipolysis, whereas endothelin-1 promotes insulin resistance and increases lipolysis [25, 26]. Given the minor contribution of neprilysin relative to angiotensin converting enzyme and aminopeptidase [27] and the proven clinical safety of sacubitril/valsartan [16], it seems unlikely that an increase in bradykinin explains the observed metabolic response. Likewise, sacubitril/valsartan was shown to decrease, not increase, endothelin-1 plasma concentrations in patients with heart failure and reduced ejection fraction. Indeed, neprilysin contributes to both endothelin-1 formation from its precursors and endothelin-1 degradation [28]. Hence, an involvement of endothelin-1 in mediation 
of metabolic effects of sacubitril/valsartan is also unlikely. Although the effect of sacubitril/valsartan on multiple other metabolically relevant neprilysin substrates has not been evaluated, its effect on the NP system, including increased NP and cyclic guanosine monophosphate levels, has been well established in nonclinical and clinical studies [17, 28, 29]. Given the strong evidence for an effect of NPs on lipolysis and glucose metabolism in conjunction with the demonstrated increase in NP and cyclic guanosine monophosphate availability with sacubitril/valsartan [17], we propose that increased NP availability may have contributed, at least in part, to metabolic improvements observed in our study.

NPs are more potent in stimulating human adipose tissue lipolysis than the prototypical $\beta$-adrenoreceptor agonist isoproterenol [9]. Due to high NP clearance receptor expression in adipose tissue of various animal species, NP-induced lipolysis is only observed in primates [30], thus limiting the utility of standard preclinical animal models. The present study, for the first time, examined the longterm effects of simultaneous neprilysin inhibition and $A T_{1}$-receptor blockade on abdominal subcutaneous and whole-body lipolysis in humans. The sustained increase in subcutaneous adipose tissue lipolysis is consistent with the observation that ex vivo adipose tissue lipolysis is not desensitized in patients with heart failure despite increased NP levels [30]. The observation that whole-body lipolysis does not increase with sacubitril/valsartan treatment suggests that increased adipose tissue lipolysis may be balanced by reduced lipolysis elsewhere in the body, however, further studies are required to

elucidate the mechanism.

In the present study, patients treated with sacubitril/valsartan tended to exhibit lower norepinephrine and epinephrine plasma concentrations compared with patients treated with amlodipine. Given the central role of catecholamines in regulation of lipid mobilization, the expected response is reduced whole-body and adipose tissue lipolysis. Instead, sacubitril/valsartan-treated patients showed increased adipose tissue lipolysis with sustained whole-body lipolysis, presumably through increased availability of neprilysin substrates.

Unopposed increases in adipose tissue lipolysis could promote ectopic fat storage and insulin resistance through accumulation of toxic lipid intermediates, including diacylglycerol [3]. However, NPs acutely increase postprandial fatty acid oxidation in humans, and circulating levels of the anti-inflammatory and insulin sensitizing adipokine adiponectin [13]. Thus far, data on metabolic effects of sustained NP activation were limited to nonclinical experiments. Nonclinical and in vitro studies have demonstrated that NP activation and transgenic overexpression enhanced mitochondrial oxidative capacity and lipid oxidation in human skeletal myotubes and in adipocytes [14], and protected mice from high-fat diet induced obesity and insulin resistance [31], respectively. Additionally, chronic NP infusion upregulated muscular oxidative capacity in obese and diabetic mice, thereby ameliorating lipotoxicity and insulin resistance [32]. The present clinical study did not indicate alterations in whole-body fat oxidation by sacubitril/valsartan treatment. We speculate that, after treatment with sacubitril/valsartan, NP-mediated changes in oxidative metabolism at the organ level that cannot be captured by indirect calorimetry may have attenuated accumulation of toxic lipid intermediates and subsequent impairment of insulin signaling [3]. It is also possible that augmented NP availability increased peripheral glucose and insulin supply through vascular 
actions. Augmenting cyclic guanosine monophosphate signaling through phosphodiesterase 5 inhibition with sildenafil also improved insulin sensitivity in overweight individuals with prediabetes [33].

Combination of state-of-the-art methodologies in a comparatively large sample size is a particular strength of our study. In fact, hyperinsulinemic euglycemic clamp testing, $\left[1,1,2,3,3-{ }^{2} \mathrm{H}\right]$-glycerol tracer kinetics, and microdialysis technics are considered gold-standards in assessing insulin sensitivity, whole-body lipolysis, and subcutaneous adipose tissue lipolysis, respectively. The total daily dose of sacubitril/valsartan used in this study $(400 \mathrm{mg})$ is known to provide superior blood pressure control in patients with arterial hypertension when administered once daily [34] and to reduce cardiovascular mortality and hospitalizations due to heart failure in patients with systolic dysfunction when administered as sacubitril/valsartan 200 mg twice daily compared with standard-of-care RAS inhibition. Therefore, the findings of this study are relevant for the future clinical use of sacubitril/valsartan.

We studied patients representing a human model for impaired insulin sensitivity. Given the close association between adiposity and risk for T2DM, and the epidemic increase in the incidence of T2DM in recent years, cardiovascular medications with added benefits on insulin sensitivity are highly desirable. Neprilysin activity seems to be upregulated in obese and insulin-resistant individuals [6], such that sacubitril/valsartan directly targets a mechanism mediating relative natriuretic peptide deficiency. Furthermore, heart failure, for which sacubitril/valsartan is approved for, is also associated with insulin resistance and muscular metabolic abnormalities. Patients with heart failure exhibit a reduction in oxidative slow twitch (type I) relative to glycolytic fast twitch (type II) muscle fibers along with reduced mitochondrial density [35]. This intrinsic impairment in skeletal muscle metabolism likely limits exercise capacity and may contribute to associated insulin resistance in patients with heart failure [36]. Decreased skeletal muscle oxidative capacity in heart failure is prevalent and clinically relevant because poor exercise tolerance and impaired insulin sensitivity predict mortality risk in these patients [37]. Therefore, metabolic improvements with sacubitril/valsartan may also be beneficial in patients with heart failure, thereby differentiating sacubitril/valsartan from currently available cardiovascular drugs.

The main limitation of our study was the fact that the change in the primary endpoint SI between interventions was not statistically significant. To account for an interim analysis conducted for SI, the $p$ value for equality of treatments was reported at $2.5 \%$ level of significance for SI, whereas it was reported at $5 \%$ level of significance for all other glucose-clamp derived measurements. Nonetheless, there was a clear trend for improvement of SI with sacubitril/valsartan compared to amlodipine. Furthermore, GIR with or without adjustment for body weight, an established marker for insulin sensitivity, was significantly improved with sacubitril/valsartan, as were all other glucose-clamp derived measurements of insulin sensitivity, supporting the overall conclusion. Moreover, the fact that we used amlodipine as the metabolically neutral comparator does not allow distinguishing individual contributions of neprilysin inhibition and angiotensin receptor blockade to the effects of sacubitril/valsartan observed in this study. Furthermore, we cannot completely rule out a carryover effect of metabolic testing, particularly insulin infusion, on lipolysis measurements obtained on the following study day. However, all patients underwent all study assessments in the same 
sequence suggesting that an impact on the comparison of investigational treatments is unlikely. Finally, our study was conducted in a relatively homogenous white population. Although there is no ethnic sensitivity with regard to exposure to LCZ696 analytes (sacubitril, sacubitrilat, and valsartan), extrapolation to other ethnic groups requires careful consideration.

\section{CONCLUSION}

In conclusion, our study demonstrated that sacubitril/valsartan treatment compared with amlodipine resulted in improved peripheral insulin sensitivity and increased abdominal subcutaneous adipose tissue lipid mobilization, but did not show significant effects on whole-body lipolysis, energy expenditure, and substrate oxidation. Moreover, our study highlights the utility of mechanistic profiling studies in discerning metabolic drug actions that could go undetected in clinical development. 


\section{METHODS}

\section{Study design and participants}

This was a multicenter, randomized, double-blind, double-dummy, activecontrolled, and parallel-group study. Eligible subjects were $\geq 18$ years of age with abdominal obesity (waist circumference $\geq 102 \mathrm{~cm}$ for men and $\geq 88 \mathrm{~cm}$ for women) and elevated blood pressure either untreated (mean seated systolic blood pressure $\geq 130 \mathrm{mmHg}$ and $<180 \mathrm{mmHg}$ at screening) or treated with up to two classes of antihypertensive therapy (mean seated systolic blood pressure $\leq 160 \mathrm{mmHg}$ at screening and $<180 \mathrm{mmHg}$ at the end of the 4-week washout period). Women had to be of nonchild-bearing potential. Key exclusion criteria were severe hypertension (mean seated diastolic blood pressure $\geq 100 \mathrm{mmHg}$ and/or mean seated systolic blood pressure $\geq 180 \mathrm{mmHg}$ at screening or at the end of the washout period), type 1 or type 2 diabetes mellitus, fasting plasma glucose $\geq 126 \mathrm{mg} / \mathrm{dL}$ or $\mathrm{HbA} 1 \mathrm{c}$ $\geq 6.5 \%$ ), dyslipidemia requiring pharmacological therapy with fibrates or nicotinic acid, concomitant use of antihypertensive medications, antidiabetic medications or drugs that may affect glucose or lipid metabolism, previous or current diagnosis of cardiac structural and functional abnormalities, history or current diagnosis of heart failure (New York Heart Association classes II-IV), history of myocardial infarction, coronary bypass surgery or percutaneous coronary intervention during the 6 months before screening, history of angioedema, or known hypersensitivity to the study drugs.

The study included a screening period of up to 4 weeks followed by a 4-week washout period and an 8-week randomized, double-blind, and double-dummy treatment phase (Figure 1). Patients receiving antihypertensive medications at the time of screening discontinued the therapy during the washout period.

In the double-blind treatment period, patients were randomized to receive sacubitril/valsartan $400 \mathrm{mg}$ q.d. or amlodipine $10 \mathrm{mg}$ q.d. along with matching placebos for 8 weeks. Patients were stratified into four groups based on the baseline Homeostasis Model Assessment of Insulin Resistance $(<2.5$ and $\geq 2.5$ ) and use of statin therapy within 8 weeks before randomization. Stratification was intended to ensure that within each stratum the number of subjects receiving either LCZ696 or amlodipine is comparable, thereby avoiding any impact of a potential imbalance in baseline characteristics on study outcome.

All participants provided written informed consent before screening. The study protocol was reviewed by the independent ethics committee or institutional review board for each center, conducted in accordance with the Declaration of Helsinki, and registered at clinicaltrials.gov under the identifier NCT01631864.

\section{Peripheral insulin sensitivity}

Changes in insulin sensitivity were measured from baseline to week 8 for sacubitril/valsartan $400 \mathrm{mg}$ q.d. vs. amlodipine $10 \mathrm{mg}$ q.d. by a hyperinsulinemiceuglycemic glucose clamp. Assessments were performed on day -1 (baseline) and on day 56 under fasting conditions. A dorsal hand vein was catheterized and kept warm for arterialized blood sampling and the contralateral arm was catheterized for glucose and insulin infusions. The procedure consisted of a 2-hour primed infusion 
of insulin (a priming dose over the first 10 minutes $\left(103 \mathrm{mU} / \mathrm{m}^{2} / \mathrm{min}\right.$ at $0-5$ minutes and $57 \mathrm{mU} / \mathrm{m}^{2} / \mathrm{min}$ at $5-10$ minutes) followed by a continuous infusion at 40 $\mathrm{mU} / \mathrm{m}^{2} / \mathrm{min}$ thereafter until 2 hours) and a variable glucose infusion to achieve steady-state plasma insulin levels while maintaining blood glucose levels at 90.1 $\mathrm{mg} / \mathrm{dL}(5.0 \mathrm{mmol} / \mathrm{L})$. Blood samples were collected continuously (automated clamp) or at approximately 5 minute intervals (manual clamp) to determine glucose levels during hyperinsulinemic- euglycemic clamp testing. The last 30 minutes (minutes 90 -120) of the clamp were considered as the steady-state period and the mean GIR was calculated for this period.

Insulin SI was calculated from steady-state GIRs and plasma insulin and glucose concentrations (SI: glucose infusion rate/(plasma glucose $x$ plasma insulin), $\mu \mathrm{g} / \mathrm{kg}^{*} \mathrm{~min} /\left(\mathrm{mmol} / \mathrm{L}^{*} \mathrm{pmol} / \mathrm{L}\right)$ ). Whole-body glucose disposal rate $(\mathrm{M}, \mathrm{mg} / \mathrm{min})$ was calculated from mean GIRs at steady state to assess peripheral insulin sensitivity. Finally, the metabolic clearance rate was calculated from the M-value and the mean blood glucose concentration at steady state (metabolic clearance rate: $\mathrm{M} / \mathrm{blood}$ glucose, min*mmol). The glucose disposal rate was expressed per unit of insulin at steady state, calculated from M-value and plasma insulin concentrations (M/l, U/min).

\section{Lipolysis}

Lipolysis was assessed on day 1 before the first dosage and on day 57 . One microdialysis probe was placed in the subcutaneous adipose tissue $6-8 \mathrm{~cm}$ lateral from the umbilicus with the patient under local anesthesia and sterile conditions, as described previously [38]. The process comprised a recovery phase of 60 minutes during which the probes were infused with perfusion solution $(50 \mathrm{mM}$ ethanol + T1 perfusion solution; CMA Microdialysis AB, Stockholm, Sweden) at a flow rate of 0.3 $\mu \mathrm{L} / \mathrm{min}$ followed by a flow calibration phase of 60 minutes. During the flow calibration phase, the perfusion rate was maintained at $0.3 \mu \mathrm{L} / \mathrm{min}$ for the first 30 minutes and increased thereafter to $2.0 \mu \mathrm{L} / \mathrm{min}$ for the remaining 30 minutes. Microdialysate samples were collected at 30 minutes and 45 minutes in the flow calibration phase.

Glycerol (an indicator of local lipolysis), glucose, and lactate concentrations were measured in microdialysates. The ethanol ratio (ratio of ethanol concentration in dialysate to ethanol concentration in perfusate) was measured as an indicator of adipose tissue blood flow.

Whole-body lipolysis was assessed using $\left[1,1,2,3,3-{ }^{2} \mathrm{H}\right]$-glycerol tracer kinetics. A glycerol tracer bolus $(3.0 \mu \mathrm{g} / \mathrm{kg})$ was injected at the start of the microdialysis flow calibration phase, followed by a continuous infusion $(0.1 \mu \mathrm{g} / \mathrm{kg} / \mathrm{min})$ until the end of the sampling period. Blood samples were collected at $0,30,40,50$, and 60 minutes during the flow calibration phase and at 15,30 , and 45 minutes thereafter. The rate of appearance of endogenous glycerol was calculated as the ratio of the glycerol tracer infusion rate to the plasma glycerol tracer enrichment. At steady state, glycerol rate of appearance was calculated from glycerol tracer enrichment using Steele's equation. 


\section{Statistical analysis}

Based on $80 \%$ power to detect a difference of 0.1 in SI at week 8 between the two treatment groups at $2.5 \%$ significance level with two-sided alternative, 90 subjects (45 in each group) were needed to complete the study. SI at 8 weeks was analyzed using the analysis of covariance model with treatment as a fixed effect and baseline insulin sensitivity as a covariate for assessing the difference in the mean effect of sacubitril/valsartan vs. amlodipine. For the purpose of a two-sided hypothesis of equality of treatment effects at $2.5 \%$ level of significance after the completion of the study, the point estimate and $97.5 \% \mathrm{Cl}$ for the difference along with the $p$-value for equality of the two treatments were reported. The smaller level of significance $(2.5 \%)$ was chosen to account for the conduct of an interim analysis. Differences between treatments for change in SI from baseline were analyzed as described above. Similar analyses at $5 \%$ level of significance were performed for all other clamp-derived measures of insulin sensitivity.

For abdominal subcutaneous adipose tissue microdialysate data (ethanol ratio, dialysate lactate, dialysate glucose, and plasma glycerol, glycerol, NEFA, glucose, insulin, adrenalin, and noradrenalin) at rest, data were analyzed using repeated measures analysis on log transformed values with treatment, visit, time and treatment ${ }^{\star}$ visit ${ }^{\star}$ time interaction as fixed effects. Ratio to day 1 (day 57 vs. day 1 ) at each treatment and ratio of sacubitril/valsartan vs. amlodipine for ratio to day 1 along with the corresponding $95 \% \mathrm{Cls}$ and $\mathrm{p}$-values are presented for 30 and 45 minute microdialysis time points combined. Comparison of 30 and 45-minute microdialysis time points demonstrated steady state of the microdialysis experiment.

Oxidative metabolism was analyzed using the analysis of covariance with treatment as fixed effect and baseline as covariate and the treatment mean difference with $95 \% \mathrm{Cls}$ and p-values are reported.

For biomarkers, data were analyzed using repeated measures of analysis on log transformed values with treatment, visit, time and treatment*visit*time interaction as fixed effects. Ratio to day 1 (day 57 vs. day 1) at each treatment and ratio of sacubitril/valsartan vs. amlodipine for ratio to day 1 along with the corresponding $95 \% \mathrm{Cls}$ and $\mathrm{p}$-values are presented. 


\section{REFERENCES}

1. Jordan J, Yumuk V, Schlaich M, Nilsson PM, Zahorska-Markiewicz B, Grassi G, et al. Joint statement of the European Association for the Study of Obesity and the European Society of Hypertension: obesity and difficult to treat arterial hypertension. J Hypertens. 2012;30(6):1047-55.

2. Del Gobbo LC, Kalantarian S, Imamura F, Lemaitre R, Siscovick DS, Psaty BM, et al. Contribution of Major Lifestyle Risk Factors for Incident Heart Failure in Older Adults: The Cardiovascular Health Study. JACC Heart Fail. 2015;3(7):520-8.

3. Samuel VT, Petersen KF, Shulman GI. Lipid-induced insulin resistance: unravelling the mechanism. Lancet. 2010;375(9733):2267-77.

4. van Hees AM, Jans A, Hul GB, Roche HM, Saris WH, Blaak EE. Skeletal muscle fatty acid handling in insulin resistant men. Obesity (Silver Spring). 2011;19(7):13509.

5. Schling $P$, Schafer $T$. Human adipose tissue cells keep tight control on the angiotensin II levels in their vicinity. J Biol Chem. 2002;277(50):48066-75.

6. Standeven KF, Hess K, Carter AM, Rice GI, Cordell PA, Balmforth AJ, et al. Neprilysin, obesity and the metabolic syndrome. Int J Obes (Lond). 2011;35(8):103140.

7. Wang TJ, Larson MG, Levy D, Benjamin EJ, Leip EP, Wilson PW, et al. Impact of obesity on plasma natriuretic peptide levels. Circulation. 2004;109(5):594-600.

8. Magnusson M, Jujic A, Hedblad B, Engstrom G, Persson M, Struck J, et al. Low plasma level of atrial natriuretic peptide predicts development of diabetes: the prospective Malmo Diet and Cancer study. J Clin Endocrinol Metab. 2012;97(2):63845.

9. Meirhaeghe A, Sandhu MS, McCarthy MI, de Groote P, Cottel D, Arveiler D, et al. Association between the T-381C polymorphism of the brain natriuretic peptide gene and risk of type 2 diabetes in human populations. Hum Mol Genet. 2007;16(11):1343-50.

10. Jujic A, Nilsson PM, Engstrom G, Hedblad B, Melander O, Magnusson M. Atrial natriuretic peptide and type 2 diabetes development--biomarker and genotype association study. PLoS One. 2014;9(2):e89201.

11. Birkenfeld AL, Boschmann M, Moro C, Adams F, Heusser K, Franke G, et al. Lipid mobilization with physiological atrial natriuretic peptide concentrations in humans. $J$ Clin Endocrinol Metab. 2005;90(6):3622-8.

12. Birkenfeld AL, Budziarek P, Boschmann M, Moro C, Adams F, Franke G, et al. Atrial natriuretic peptide induces postprandial lipid oxidation in humans. Diabetes. 2008;57(12):3199-204.

13. Coue M, Badin PM, Vila IK, Laurens C, Louche K, Marques MA, et al. Defective Natriuretic Peptide Receptor Signaling in Skeletal Muscle Links Obesity to Type 2 Diabetes. Diabetes. 2015;64(12):4033-45.

14. Engeli S, Birkenfeld AL, Badin PM, Bourlier V, Louche K, Viguerie N, et al. Natriuretic peptides enhance the oxidative capacity of human skeletal muscle. J Clin Invest. 2012;122(12):4675-9.

15. van der Zijl NJ, Moors CC, Goossens GH, Hermans MM, Blaak EE, Diamant M. Valsartan improves \{beta\}-cell function and insulin sensitivity in subjects with impaired glucose metabolism: a randomized controlled trial. Diabetes Care. 2011;34(4):845-51.

16. McMurray JJ, Packer M, Desai AS, Gong J, Lefkowitz MP, Rizkala AR, et al. Angiotensin-neprilysin inhibition versus enalapril in heart failure. $\mathrm{N}$ Engl $\mathrm{J}$ Med. 2014;371(11):993-1004. 
17. Langenickel T.H. DWP. Angiotensin receptor-neprilysin inhibition with LCZ696: a novel approach for the treatment of heart failure. Drug Discovery Today: Therapeutic Strategies. 2012;9(4):e131-e9.

18. Hall JE, do Carmo JM, da Silva AA, Wang Z, Hall ME. Obesity-induced hypertension: interaction of neurohumoral and renal mechanisms. Circ Res. 2015;116(6):991-1006.

19. Morris MJ. Cardiovascular and metabolic effects of obesity. Clin Exp Pharmacol Physiol. 2008;35(4):416-9.

20. Jing $\mathrm{F}$, Mogi M, Horiuchi M. Role of renin-angiotensin-aldosterone system in adipose tissue dysfunction. Mol Cell Endocrinol. 2013;378(1-2):23-8.

21. Navigator Study Group, McMurray JJ, Holman RR, Haffner SM, Bethel MA, Holzhauer B, et al. Effect of valsartan on the incidence of diabetes and cardiovascular events. N Engl J Med. 2010;362(16):1477-90.

22. Goossens GH. The renin-angiotensin system in the pathophysiology of type 2 diabetes. Obes Facts. 2012;5(4):611-24.

23. Wang TJ, Larson MG, Keyes MJ, Levy D, Benjamin EJ, Vasan RS. Association of plasma natriuretic peptide levels with metabolic risk factors in ambulatory individuals. Circulation. 2007;115(11):1345-53.

24. Boschmann M, Engeli S, Adams F, Franke G, Luft FC, Sharma AM, et al. Influences of AT1 receptor blockade on tissue metabolism in obese men. Am J Physiol Regul Integr Comp Physiol. 2006;290(1):R219-23.

25. Eriksson AK, van Harmelen V, Stenson BM, Astrom G, Wahlen K, Laurencikiene J, et al. Endothelin-1 stimulates human adipocyte lipolysis through the ET A receptor. Int $\mathrm{J}$ Obes (Lond). 2009;33(1):67-74.

26. Mori MA, Sales VM, Motta FL, Fonseca RG, Alenina N, Guadagnini D, et al. Kinin B1 receptor in adipocytes regulates glucose tolerance and predisposition to obesity. PLoS One. 2012;7(9):e44782.

27. Fryer RM, Segreti J, Banfor PN, Widomski DL, Backes BJ, Lin CW, et al. Effect of bradykinin metabolism inhibitors on evoked hypotension in rats: rank efficacy of enzymes associated with bradykinin-mediated angioedema. $\mathrm{Br} \mathrm{J}$ Pharmacol. 2008;153(5):947-55.

28. Kobalava Z, Kotovskaya Y, Averkov O, Pavlikova E, Moiseev V, Albrecht D, et al. Pharmacodynamic and Pharmacokinetic Profiles of Sacubitril/Valsartan (LCZ696) in Patients with Heart Failure and Reduced Ejection Fraction. Cardiovasc Ther. 2016;34(4):191-8.

29. Gu J, Noe A, Chandra P, Al-Fayoumi S, Ligueros-Saylan M, Sarangapani R, et al. Pharmacokinetics and pharmacodynamics of LCZ696, a novel dual-acting angiotensin receptor-neprilysin inhibitor (ARNi). J Clin Pharmacol. 2010;50(4):40114.

30. Birkenfeld AL, Adams F, Schroeder C, Engeli S, Jordan J. Metabolic actions could confound advantageous effects of combined angiotensin II receptor and neprilysin inhibition. Hypertension. 2011;57(2):e4-5.

31. Miyashita $\mathrm{K}$, Itoh $\mathrm{H}$, Tsujimoto $\mathrm{H}$, Tamura $\mathrm{N}$, Fukunaga $\mathrm{Y}$, Sone $\mathrm{M}$, et al. Natriuretic peptides/cGMP/cGMP-dependent protein kinase cascades promote muscle mitochondrial biogenesis and prevent obesity. Diabetes. 2009;58(12):2880-92.

32. Sengenes C, Zakaroff-Girard A, Moulin A, Berlan M, Bouloumie A, Lafontan M, et al. Natriuretic peptide-dependent lipolysis in fat cells is a primate specificity. Am $\mathrm{J}$ Physiol Regul Integr Comp Physiol. 2002;283(1):R257-65.

33. Ramirez CE, Nian H, Yu C, Gamboa JL, Luther JM, Brown NJ, et al. Treatment with Sildenafil Improves Insulin Sensitivity in Prediabetes: A Randomized, Controlled Trial. J Clin Endocrinol Metab. 2015;100(12):4533-40.

34. Kario K, Sun N, Chiang FT, Supasyndh O, Baek SH, Inubushi-Molessa A, et al. Efficacy and safety of LCZ696, a first-in-class angiotensin receptor neprilysin 
inhibitor, in Asian patients with hypertension: a randomized, double-blind, placebocontrolled study. Hypertension. 2014;63(4):698-705.

35. Drexler H, Riede U, Munzel T, Konig H, Funke E, Just H. Alterations of skeletal muscle in chronic heart failure. Circulation. 1992;85(5):1751-9.

36. Kemppainen J, Tsuchida H, Stolen K, Karlsson H, Bjornholm M, Heinonen OJ, et al. Insulin signalling and resistance in patients with chronic heart failure. J Physiol. 2003;550(Pt 1):305-15.

37. Doehner W, Rauchhaus M, Ponikowski P, Godsland IF, von Haehling S, Okonko DO, et al. Impaired insulin sensitivity as an independent risk factor for mortality in patients with stable chronic heart failure. J Am Coll Cardiol. 2005;46(6):1019-26.

38. Goossens GH, Blaak EE, Saris WH, van Baak MA. Angiotensin II-induced effects on adipose and skeletal muscle tissue blood flow and lipolysis in normal-weight and obese subjects. J Clin Endocrinol Metab. 2004;89(6):2690-6. 


\section{SUPPLEMENTARY MATERIAL}

\section{Energy Expenditure and Substrate Oxidation}

Energy expenditure and substrate oxidation were assessed in parallel by indirect calorimetry using a ventilated hood system. The ventilated hood measurements were recorded for 30 minutes in the resting state (during the flow calibration phase) and averaged for further analysis. Resting energy expenditure (REE) was calculated using the abbreviated Weir equation $(\mathrm{REE}=[3.9(\mathrm{VO} 2)+1.1(\mathrm{VCO})]$ 1.44) (with VO2 and VCO2 expressed as $\mathrm{mL} / \mathrm{min}$ ).

\section{Circulating metabolites, insulin, and catecholamines}

Fasting plasma concentrations of free fatty acids, glycerol, glucose, insulin, epinephrine, and norepinephrine were evaluated at baseline (Day 1) and on Day 57 at rest and concurrently with the microdialysis experiment. Blood samples were taken at 30 minutes and 45 minutes following the calibration flow phase.

\section{Blood Pressure}

Office BP was measured at screening, during washout and throughout the study at baseline, week 4, week 8 and end of study using the same arm and the same automated equipment with an appropriate cuff size. Measurements were performed in triplicates at 2-minute intervals after patients have been sitting for 15 minutes with the back supported and both feet on the floor. During the home stay period, patients were given a home measurement device and instructed to monitor BP twice weekly at approximately the same time each morning (7-9 AM).

\section{Safety Assessments}

Safety assessments included adverse events (AEs) and serious adverse events (SAEs) and were regularly monitored throughout the study based on hematology, blood chemistry and urine analysis as well as assessments of vital signs (BP and pulse measurements), ECG, physical condition and body weight. 


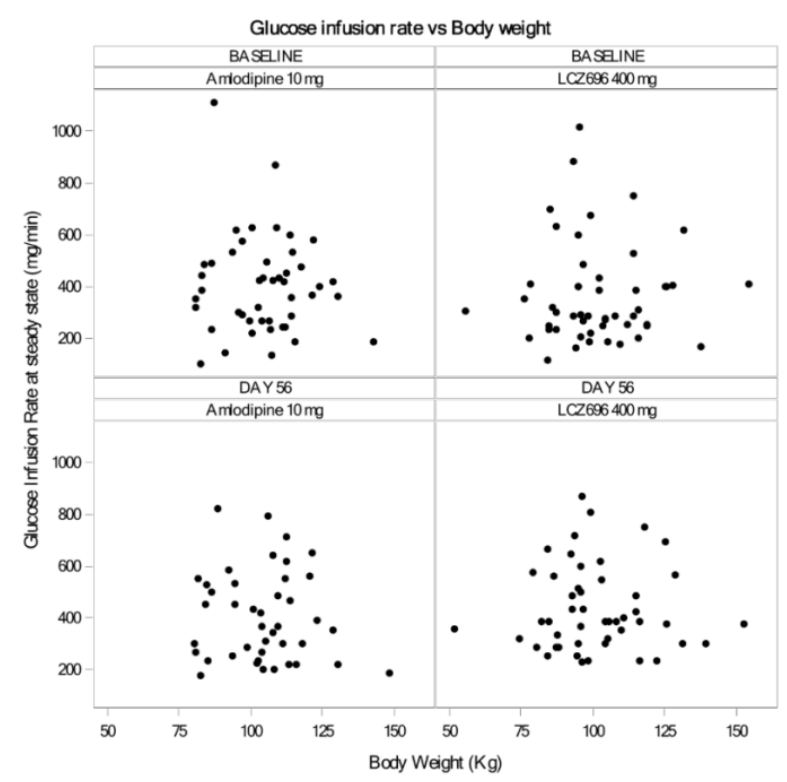

Supplementary Figure 1A. Comparison of glucose infusion rate with body weight at baseline and Day 56.

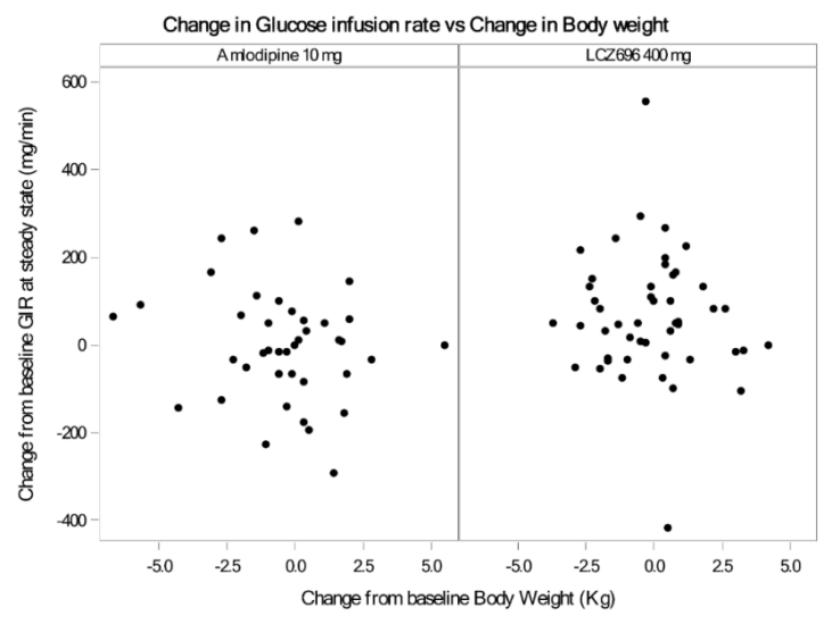

Supplementary Figure 1B. Change from baseline in glucose infusion rate vs. body weight following 8-week treatment with LCZ696 vs. amlodipine 
Supplementary Table 1. Mean Change in Biomarkers from Baseline to Day 57

\begin{tabular}{|c|c|c|c|}
\hline $\begin{array}{c}\text { Biomarker } \\
\text { Adjusted means } \\
(95 \% \mathrm{Cls})\end{array}$ & $\begin{array}{c}\text { LCZ696 } \\
(400 \mathrm{mg} \text { q.d. }) \\
(\mathrm{n}=50)\end{array}$ & $\begin{array}{c}\text { Amlodipine } \\
(10 \mathrm{mg} \text { q.d. }) \\
(\mathrm{n}=46)\end{array}$ & $\begin{array}{l}\text { Treatment difference } \\
\text { (LCZ696 vs. } \\
\text { Amlodipine) }\end{array}$ \\
\hline \multicolumn{4}{|l|}{ Glucose (mmol/L) } \\
\hline Baseline & $5.64(5.51,5.78)$ & $5.52(5.39,5.65)$ & - \\
\hline Day 57 & $5.66(5.55,5.78)$ & $5.50(5.38,5.62)$ & - \\
\hline Ratio to baseline & $1.00(0.99,1.02)$ & $1.00(0.98,1.01)$ & $1.01(0.98,1.03)$ \\
\hline \multicolumn{4}{|l|}{ Insulin (pmol/L) } \\
\hline Baseline & $41.09(34.68,48.67)$ & $37.54(31.45,44.79)$ & \\
\hline Day 57 & $41.07(34.57 ; 48.79)$ & $37.91(31.61,45.47)$ & \\
\hline Ratio to baseline & $1.00(0.88,1.14)$ & $1.01(0.88 ; 1.16)$ & $0.99(0.82,1.20)$ \\
\hline \multicolumn{4}{|l|}{ Glycerol ( $\mu \mathrm{mol} / \mathrm{L})$} \\
\hline Baseline & 85.39 (77.97; 93.52) & $91.61(83.32,100.73)$ & \\
\hline Day 57 & $84.48(76.85,92.87)$ & $87.90(79.51,97.18)$ & \\
\hline Ratio to baseline & $0.99(0.91,1.08)$ & $0.96(0.87 ; 1.05)$ & $1.03(0.91,1.17)$ \\
\hline \multicolumn{4}{|c|}{ Non-esterified fatty acids (mmol/L) } \\
\hline $\begin{array}{l}\text { Baseline, adjusted } \\
\text { mean }\end{array}$ & $0.63(0.56,0.70)$ & $1.03(0.91,1.17)$ & \\
\hline Day 57 & $0.60(0.53,0.68)$ & $1.03(0.91,1.17)$ & \\
\hline Ratio to baseline & $0.96(0.86,1.06)$ & $0.92(0.82,1.03)$ & $1.04(0.89,1.21)$ \\
\hline \multicolumn{4}{|c|}{ Epinephrine (pg/mL) } \\
\hline Baseline & $25.23(21.20,30.03)$ & $20.98(17.49,25.16)$ & \\
\hline Day 57 & $20.42(17.19,24.25)$ & $21.91(18.28,26.26)$ & \\
\hline Ratio to baseline & $0.81(0.72,0.91)^{a}$ & $1.04(0.93,1.18)$ & $0.77(0.65,0.91)^{b}$ \\
\hline \multicolumn{4}{|c|}{ Norepinephrine $(\mathrm{pg} / \mathrm{mL})$} \\
\hline Baseline, & $217.3(188.3,250.7)$ & $230.8(198.8 ; 267.9)$ & \\
\hline Day 57 & $236.8(204.2,274.5)$ & $276.2(236.4 ; 322.9)$ & \\
\hline Ratio to baseline & $1.09(0.98,1.21)$ & $1.20(1.07,1.33)^{\mathrm{C}}$ & $0.91(0.78,1.06)$ \\
\hline
\end{tabular}

${ }^{a} p<0.001 ;{ }^{b} p=0.002 ;{ }^{c} p=0.001$

Data was analyzed using repeated measurements on log transformed values with treatment, visit, time and treatment ${ }^{\star}$ visit ${ }^{\star}$ time interaction as fixed effect assuming an Unstructured covariance. Values are then log back transformed. 
Supplementary Table 2. Incidence of AE occurring in more than one patient safety analysis set

\begin{tabular}{|c|c|c|}
\hline & $\begin{array}{c}\text { LCZ696 400 } \mathbf{~ m g} \\
\mathbf{N}=\mathbf{5 0}, \mathbf{n}(\%)\end{array}$ & $\begin{array}{c}\text { Amlodipine 10 mg } \\
\mathbf{N}=\mathbf{3 8}, \mathbf{n}(\%)\end{array}$ \\
\hline Subjects with AEs, $\mathrm{n}(\%)$ & $30(60.0)$ & $37(77.1)$ \\
\hline Nasopharyngitis & $9(18.0)$ & $8(16.7)$ \\
\hline Peripheral edema & $1(2.0)$ & $16(33.3)$ \\
\hline Headache & $4(8.0)$ & $6(12.5)$ \\
\hline Pruritus & $5(10.0)$ & - \\
\hline Diarrhea & $3(6.0)$ & $1(2.1)$ \\
\hline Dizziness & $3(6.0)$ & $1(2.1)$ \\
\hline Pollakisuria & $2(4.0)$ & $2(4.2)$ \\
\hline Circulatory collapse* & - & $1(2.1)$ \\
\hline Fatigue & $1(2.0)$ & - \\
\hline Gastroenteritis & $2(4.0)$ & - \\
\hline Orthostatic hypotension & $2(4.0)$ & $1(2.1)$ \\
\hline Pruritus generalized & $2(4.0)$ & - \\
\hline Toothache & $1(2.0)$ & - \\
\hline
\end{tabular}

AE, adverse event

*Vasovagal reaction coded as circulatory collapse according to MedDRA 
CHAPTER 4 


\section{Effect of sacubitril/valsartan on exercise induced lipid metabolism in individuals with obesity and hypertension}

Engeli S., Stinkens R., Heise T., May M., Goossens G.H., Blaak E.E., Jax T., Albrecht D., Pal P., Tegtbur U., Haufe S., Langenickel T.H., Jordan J.

Submitted 


\section{ABSTRACT}

Background: sacubitril/valsartan (LCZ696), a novel angiotensin receptor-neprilysin inhibitor, was recently approved for the treatment of heart failure with reduced ejection fraction. Neprilysin degrades several peptides that modulate lipid metabolism, including natriuretic peptides. In this study, we investigated the effects of 8 weeks' treatment with sacubitril/valsartan on whole body and adipose tissue lipolysis and lipid oxidation during defined physical exercise compared with the metabolically neutral comparator amlodipine.

Methods: This was a multicenter, randomized, double-blind, active-controlled, parallel-group study enrolling subjects with abdominal obesity and moderate hypertension (mean sitting systolic blood pressure [msSBP] $\geq 130-180 \mathrm{mmHg}$ ). Lipolysis during rest and exercise was assessed by microdialysis and [1,1,2,3,3$\left.{ }^{2} \mathrm{H}\right]$-glycerol tracer kinetics. Energy expenditure and substrate oxidation were measured simultaneously using indirect calorimetry. Plasma non-esterified fatty acids, glycerol, insulin, glucose, adrenaline and noradrenaline concentrations, blood pressure and heart rate were also determined.

Results: Exercise elevated plasma glycerol, free fatty acids and interstitial glycerol concentrations and increased the rate of glycerol appearance. However, exerciseinduced stimulation of lipolysis was not augmented upon sacubitril/valsartan treatment compared with amlodipine treatment. Furthermore, sacubitril/valsartan did not alter energy expenditure and substrate oxidation during exercise compared with amlodipine treatment.

Conclusion: Sacubitril/valsartan treatment for 8 weeks did not elicit clinically relevant changes in exercise-induced lipolysis or substrate oxidation in obese patients with hypertension, implying that its beneficial cardiovascular effects cannot be explained by changes in lipid metabolism during exercise. 


\section{INTRODUCTION}

Fatty acids are stored in the form of triglycerides in the adipose tissue and are released during lipolysis to fuel lipid oxidation in energy consuming tissues. Lipolysis and skeletal muscle lipid oxidation decrease following carbohydrate ingestion and increase in the fasting state or during physical exercise [1]. An imbalance between fatty acid mobilization and utilization may adversely affect cardiovascular and metabolic health. Acute experimental increases in circulating fatty acids in humans worsened hepatic [2] and skeletal muscle [3] insulin sensitivity and endothelium-mediated vasodilation [4]. Chronic increase in fatty acid availability promotes hepatic, skeletal muscle and myocardial lipotoxicity, dyslipidemia, insulin resistance, and type 2 diabetes mellitus [5, 6]. Conversely, interventions that reduce fatty acid levels, improve metabolic health [5]. These observations are highly relevant for cardiovascular medications with the potential to affect lipid turnover. Sacubitril/valsartan, comprising a novel neprilysin inhibitor prodrug sacubitril and angiotensin receptor blocker (valsartan) has been approved for the treatment of chronic heart failure (NYHA Class II-IV) with reduced ejection fraction [7]. The endopeptidase neprilysin is ubiquitously expressed, including in human adipocytes, and degrades multiple peptides such as natriuretic peptides (NPs), angiotensin II, bradykinin and endothelin that may modulate lipid metabolism [8, 9]. Notably, NPs potently augment human adipose tissue lipolysis, postprandial lipid oxidation, and skeletal muscle oxidative capacity [9], whereas, angiotensin II elicits more subtle changes in fatty acid turnover [10].

Given the role and association of aberrant NP- and renin-angiotensin-aldosterone signaling in cardiovascular diseases and metabolic dysfunction, we hypothesized that simultaneous blockade of angiotensin receptor and neprilysin with sacubitril/valsartan can potentially ameliorate metabolic dysfunction, especially lipid-turnover, compared with amlodipine. In the present study, we investigated the effects of 8-weeks treatment with sacubitril/valsartan compared with the metabolically neutral comparator amlodipine on whole-body and adipose tissue lipolysis, energy expenditure and substrate oxidation during defined physical exercise, which is known to stimulate NP release and induces lipolysis and lipid oxidation.

\section{METHODS}

\section{Study design}

The study design, key inclusion and exclusion criteria of the patients and the primary results of this study have been described earlier [11]. Briefly, this was a multicenter, randomized, double-blind, double-dummy, active-controlled, parallelgroup study enrolling adult subjects with abdominal obesity (waist circumference $\geq 102 \mathrm{~cm}$ for men and $\geq 88 \mathrm{~cm}$ for women) and moderate hypertension (mean sitting systolic blood pressure [msSBP] $\geq 130$ and $<180 \mathrm{mmHg}$ ). Key exclusion criteria were severe hypertension (msSBP $>180 \mathrm{mmHg}$ ), type 1 or 2 diabetes (fasting plasma glucose $\geq 126 \mathrm{mg} / \mathrm{dL}$ or $\mathrm{HbA} 1 \mathrm{c} \geq 6.5 \%$ ), dyslipidemia requiring therapy with fibrates or nicotinic acid, concomitant use of antihypertensive, anti-diabetic or other 
medications that affect glucose and/or lipid metabolism, and a history or current diagnosis of heart failure (NYHA class II-IV).

The study included a screening period of up to 4-weeks followed by a 4-week washout period and an 8-week, randomized, double-blind, double-dummy treatment phase. Patients receiving antihypertensive medications at the time of screening discontinued the therapy during the washout period. During the treatment period, patients were randomized to receive either sacubitril/valsartan $400 \mathrm{mg}$ every day (QD) or amlodipine $10 \mathrm{mg}$ QD along with a matching placebo for 8 weeks. Patients were stratified into 4 groups based on baseline homeostatic model assessment of insulin resistance (HOMA-IR) and statin use.

All patients provided written informed consent before screening. The clinical study protocol was reviewed and approved by the Independent Ethics Committee or Institutional Review Board at each center and, conducted in accordance with declaration of Helsinki and registered at clinicaltrials.gov under the identifier NCT01631864.

\section{Exercise test}

An incremental exercise test on a bicycle ergometer was conducted before the start of the intervention (Day -14) to determine the maximal aerobic capacity $\left(\mathrm{VO}_{2}\right.$ peak) at volitional exhaustion by measuring the individual maximum workload prior to stopping for exhaustion or until pre-defined heart rate or blood pressure (BP) criteria were met. At baseline (Day 1) and after 8 weeks (Day 57), subjects exercised at $50 \%$ of $\mathrm{VO}_{2}$ peak (as determined on Day -14) for a period of $60 \mathrm{~min}$.

\section{Measurement of lipolysis}

Local adipose tissue and whole body lipolysis were assessed at baseline and after 8 weeks of treatment, as described previously [11]. Local adipose tissue lipolysis was measured by microdialysis and assessed during a 45 min interval at rest, followed by a 60 min interval during which the patients exercised at $50 \%$ of their individual $\mathrm{VO}_{2}$ peak. Dialysates were collected from abdominal subcutaneous adipose tissue at the lower right abdominal quadrant at rest and at 15-min intervals during exercise. Concentrations of glycerol (as an indicator of lipolysis), glucose and lactic acid in dialysates were measured. The ethanol outflow/inflow ratio (ratio of ethanol concentration in the dialysate and the perfusate) was measured as an indicator of adipose tissue blood flow.

Whole body lipolysis was estimated using $\left[1,1,2,3,3-{ }^{2} \mathrm{H}\right]$-glycerol tracer kinetics following an intravenous glycerol bolus $\left(2 \mu \mathrm{mol} \cdot \mathrm{kg}^{-1}\right)$ after insertion of the microdialysis catheter, approximately 60-min before the baseline measurements started, and subsequent infusion at an infusion rate of $0.1 \mu \mathrm{mol} \cdot \mathrm{kg}^{-1} \mathrm{~min}^{-1}$ at rest and $0.2 \mu \mathrm{mol} \cdot \mathrm{kg}^{-1} \mathrm{~min}^{-1}$ during exercise. Blood samples were collected at $15-\mathrm{min}$ intervals at rest and during exercise. The rate of appearance ( $\mathrm{Ra}$ ) of endogenous glycerol was calculated as the ratio of glycerol tracer infusion rate to plasma glycerol tracer enrichment. At steady state, glycerol Ra was calculated from glycerol enrichment using Steele's equation. 


\section{Energy expenditure and substrate oxidation}

Energy expenditure and substrate oxidation during rest and exercise were assessed by indirect calorimetry using a ventilated hood system. The ventilated hood measurements were recorded for 30 minutes in the resting phase with the patient in supine position and during the last 10 minutes of the 60 -min exercise period.

\section{Circulating metabolites and hormones}

Samples for fasting plasma biomarkers (non-esterified fatty acid [NEFA], glycerol, glucose, insulin, adrenaline and noradrenaline) were collected at baseline (Day 1) and on Day 57 at rest and during exercise concurrently with microdialysis measurements.

\section{Blood pressure}

Office BP was measured at screening, during washout and throughout the study at baseline, Week 4, Week 8 and at the end of study using the same automated equipment with an appropriate cuff size. Measurements were performed in triplicate at 2-min intervals after patients had been sitting for 15 minutes with the back supported and both feet on the floor. BP was also measured during the exercise phase. During the home stay period, patients were given a home measurement device and instructed to monitor BP twice weekly at approximately the same time each morning.

\section{Statistical analysis}

Following 8 weeks of treatment with sacubitril/valsartan or amlodipine, assessments of local adipose tissue lipolysis, whole body lipolysis, oxidative metabolism, BP and biomarkers during exercise were performed as pre-specified study objectives.

For abdominal subcutaneous adipose tissue microdialysate data (ethanol ratio, dialysate lactate, dialysate glucose, dialysate glycerol), plasma biomarkers (glycerol, NEFA, glucose, insulin, adrenaline and noradrenaline) and whole-body lipolysis (rate of glycerol appearance) data for 45 minutes at rest and 4 time-points during exercise $(15,30,45$ and 60 minutes) were analyzed using repeatedmeasures analysis on log-transformed values with treatment, visit, time and treatment ${ }^{*}$ visit ${ }^{*}$ time interaction as fixed effects. Geometric mean ratios of each exercise time-point to 45 minutes resting for each day and treatment, ratios of Day 57 to Day 1 for each treatment and each exercise time-point, and the ratio between sacubitril/valsartan and amlodipine for Day 57 to Day 1 were calculated.

Oxidative metabolism was analyzed using analysis of covariance (ANCOVA) with treatment as the fixed effect and baseline as the covariate. Oxidative metabolism during exercise was analyzed using analysis of variance (ANOVA) for repeated measurements with treatment, visit and treatment ${ }^{\star}$ visit interaction as fixed effects. Mean difference to Day1 (Day 57 vs. Day 1) for each treatment along with the corresponding $95 \% \mathrm{Cls}$ and $p$-values are presented. Data for exercise and resting phase were analyzed for each day and treatment with a mixed-effects linear model with phase (exercise or resting) as the fixed effect and subject as the random effect 
to obtain the mean difference estimate and $95 \% \mathrm{Cl}$ for exercise vs. rest comparison. Respiratory quotient $\left(\mathrm{CO}_{2} / \mathrm{O}_{2}\right.$ ratio) was calculated at each of the Days 1 and 57 at rest and during exercise. A statistical comparison of the quotients was made between rest and exercise within each day.

\section{RESULTS}

\section{Exercise testing}

On Day 1, 39 patients in the sacubitril/valsartan group and 24 patients randomized to the amlodipine group completed the constant workload exercise for 60 minutes. On Day 57, 36 patients treated with sacubitril/valsartan and 23 treated with amlodipine completed the exercise for 60 minutes. Similar observations were made in patients completing only 45 and 60 minutes of exercise, suggesting that 8 weeks of treatment of patients with obesity and hypertension with sacubitril/valsartan or amlodipine did not have any clinically relevant impact on the exercise duration. Oxygen consumption and workload were comparable between Day 1 and Day 57 in both treatment groups (Supplementary Table 1).

\section{Plasma glucose and insulin concentrations}

With exercise, plasma glucose concentrations increased in the amlodipine group for all time-points and for $30(p=0.017), 45(p=0.002)$ and $60(p<0.001)$ minutes in the sacubitril/valsartan group on Day 1. On Day 57 , the increase was significant during $60 \mathrm{~min}$ of exercise in the sacubitril/valsartan group $(p=0.031)$ but the difference was not significant at any time point in the amlodipine group. A decrease in glucose concentration was noticed on Day 57 in both the treatment groups as compared to baseline (Day 1), with the difference being significant only in the amlodipine group at $30(p=0.017)$ and $45(p<0.001)$ minutes of exercise. However, no statistically significant differences in glucose concentrations were observed between the treatment groups at any time-point.

A decrease in insulin concentrations with increasing exercise duration was observed in both treatment groups. When compared with resting insulin concentrations, a significant decrease was observed at $45 \mathrm{~min}(p=0.015)$ and 60 $\min (p<0.001)$ on Day 1 and at $45 \min (p=0.044)$ on Day 57 in the sacubitril/valsartan group. However, exercise-induced decreases in insulin concentrations were not statistically significant in the amlodipine group, either on Day 1 or Day 57 . After 8 weeks of treatment, compared with baseline, insulin concentrations were significantly lower in the amlodipine group at all time-points except 60 minutes, while the change was not significant at any time-point in the sacubitril/valsartan group. Significant differences in insulin concentrations were observed at $30 \mathrm{~min}(p=0.017)$ and $45 \mathrm{~min}(p=0.027)$ between treatment groups on Day 57 compared to baseline. 


\section{Subcutaneous adipose tissue lipolysis during exercise}

Compared with resting measurements, microdialysate glycerol concentrations increased during exercise, indicating increased subcutaneous adipose tissue lipolysis in both the amlodipine and sacubitril/valsartan groups on Day 1 as well as on Day 57. Compared with baseline, microdialysate glycerol concentrations during exercise were numerically lower in the amlodipine group on Day 57 . In the sacubitril/valsartan group, microdialysate glycerol concentrations increased similarly at the beginning and at the end of treatment, but this increase was not statistically significant (Figure 1). Microdialysate glucose concentrations were comparable between sacubitril/valsartan and amlodipine at baseline [sacubitril/valsartan vs. amlodipine: $15 \mathrm{~min}$ ( $1.07 \mathrm{vs.} 0.94 \mathrm{mmol} / \mathrm{L}) ; 30 \mathrm{~min}(1.06 \mathrm{vs}$. $1.02 \mathrm{mmol} / \mathrm{L}) ; 45 \mathrm{~min}(1.05 \mathrm{vs} 0.99 \mathrm{mmol} / \mathrm{L}) ; 60 \mathrm{~min}(1.03 \mathrm{vs} .0 .91 \mathrm{mmol} / \mathrm{L})]$ and on Day 57 [15 min (1.12 vs. $0.95 \mathrm{mmol} / \mathrm{L}) ; 30 \mathrm{~min}(1.08 \mathrm{vs} .0 .94 \mathrm{mmol} / \mathrm{L}) ; 45 \mathrm{~min}(1.07$ vs $1.02 \mathrm{mmol} / \mathrm{L}) ; 60 \mathrm{~min}(1.06 \mathrm{vs}$. $1.01 \mathrm{mmol} / \mathrm{L})]$. No statistically significant differences in glucose levels from baseline to Week 8 were observed between the two treatment groups. A similar trend was observed with lactate levels.

Ethanol ratios were comparable between sacubitril/valsartan and amlodipine on Day 1 [sacubitril/valsartan vs. amlodipine: $15 \mathrm{~min}$ (0.42 vs. 0.43 ); $30 \mathrm{~min}(0.42$ vs. 0.44 ); $45 \mathrm{~min}$ ( 0.43 vs 0.45 ); $60 \mathrm{~min}$ (0.47 vs. 0.46 )]. Ethanol ratios increased on Day 57 in both the sacubitril/valsartan and amlodipine groups, but remained comparable between treatment groups [15 min ( 0.49 vs. 0.49$) ; 30$ min ( 0.51 vs. $0.49) ; 45 \mathrm{~min}$ ( 0.53 vs. 0.51 ); $60 \mathrm{~min}$ ( 0.55 vs. 0.53 )]. These data suggest that there was no relevant change in blood flow that needs to be accounted for when interpreting glycerol measurements.

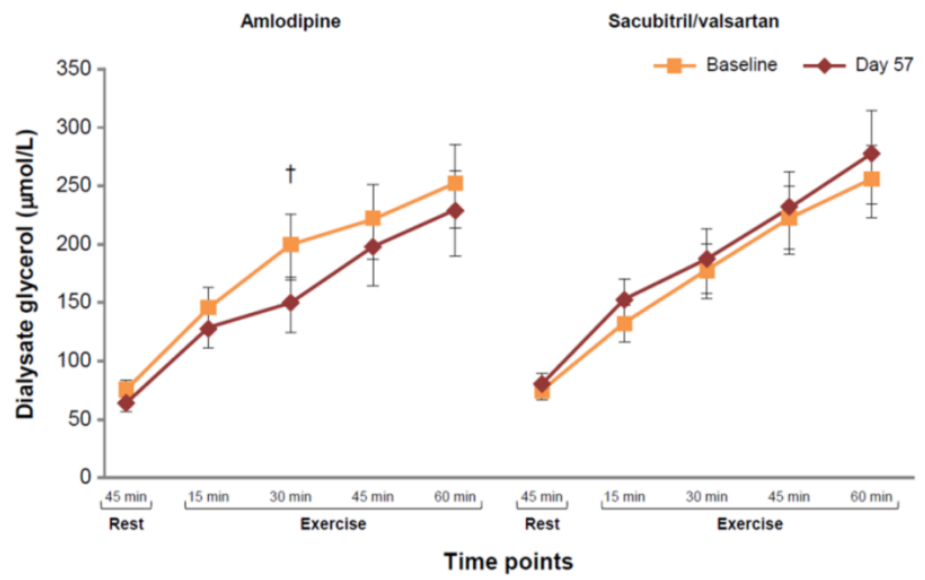

$\dagger p=0.003$ vs. baseline

Figure 1. Comparison of local adipose tissue lipolysis (dialysate glycerol) variable during exercise following 8-weeks of treatment with sacubitril/valsartan and amlodipine

Error bars indicate $95 \% \mathrm{Cl}$ 


\section{Whole-body lipolysis}

Plasma glycerol concentrations increased with exercise in both treatment groups, both on Day 1 and Day 57 [amlodipine group, Day 1 vs. Day 57: resting (89.77 vs. $88.04 \mu \mathrm{mol} / \mathrm{L}) ; 15 \mathrm{~min}(141.12$ vs. $119.56 \mu \mathrm{mol} / \mathrm{L}) ; 30 \mathrm{~min}$ (184.78 vs. 156.03 $\mu \mathrm{mol} / \mathrm{L}) ; 45 \mathrm{~min}$ (216.04 vs. $179.27 \mu \mathrm{mol} / \mathrm{L}) ; 60 \mathrm{~min}$ (224.85 vs. $191.95 \mu \mathrm{mol} / \mathrm{L})$ and sacubitril/valsartan, Day 1 vs. Day 57: resting (85.64 vs. $83.93 \mu \mathrm{mol} / \mathrm{L}) ; 15 \mathrm{~min}$ (139.3 vs. $126.92 \mu \mathrm{mol} / \mathrm{L}) ; 30 \mathrm{~min}(177.65$ vs. $157.29 \mu \mathrm{mol} / \mathrm{L}) ; 45 \mathrm{~min}(205.68$ vs. $189.84 \mu \mathrm{mol} / \mathrm{L}) ; 60 \mathrm{~min}(225.62$ vs. $205.26 \mu \mathrm{mol} / \mathrm{L})]$. Compared to baseline, plasma glycerol levels were lower in both the treatment groups on Day 57 . While the change from baseline to Day 57 was significant at all time-points in the amlodipine group $(p<0.05)$, it was significant at 30 minutes in the sacubitril/valsartan group $(p=0.012)$. The differences in plasma glycerol levels between treatment groups were not significant.

As compared with glycerol Ra following 45 minutes rest, a significant increase was observed during exercise at all time-points in both treatment groups on Day 1 and Day $57(p<0.001)$. The change from baseline to Day 57 was statistically significant in the sacubitril/valsartan group at $15 \mathrm{~min}(\mathrm{p}=0.026), 30 \mathrm{~min}(\mathrm{p}=0.012)$ and $45 \mathrm{~min}$ $(p=0.035$ ), but was not significant at any time-point in the amlodipine group (Figure 2A). However, there was no significant difference between treatment groups at any time-point.

Plasma NEFA concentrations decreased on Day 57 at 15 minutes in the sacubitril/valsartan group $(p=0.018)$ and at $15 \mathrm{~min}$ and $30 \mathrm{~min}(p<0.05)$ in the amlodipine group. No significant differences were observed between treatment groups. When compared with NEFA levels at rest (45 minutes), the levels were lower during the initial phases of exercise, but increased gradually with increasing exercise duration in both treatment groups (Figure 2B). 
A

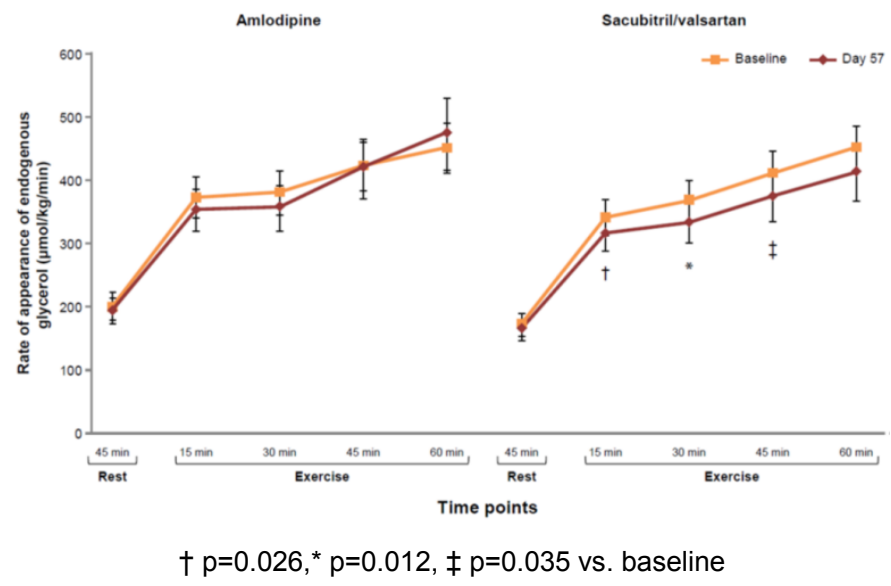

B

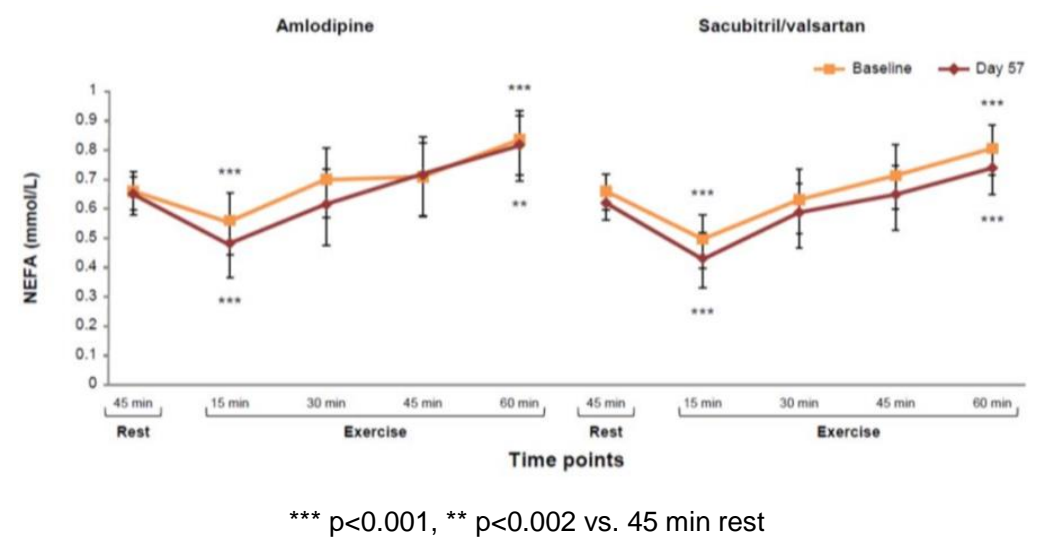

Figure 2. Whole body lipolysis at exercise: comparison of rate of glycerol appearance between treatments $(\mathbf{A})$ and plasma concentration of NEFA (B).

Error bars indicate $95 \% \mathrm{Cl}$ 


\section{Oxidative metabolism during exercise}

Oxygen consumption was comparable between the sacubitril/valsartan and amlodipine groups at baseline $\left(\mathrm{O}_{2}\right.$ consumption: amlodipine, $1.31 \pm 0.45 \mathrm{~L} / \mathrm{min}$; sacubitril/valsartan, $1.40 \pm 0.41 \mathrm{~L} / \mathrm{min}$ ) and on Day 57 (amlodipine, $1.27 \pm 0.39$ $\mathrm{L} / \mathrm{min}$; sacubitril/valsartan, $1.37 \pm 0.44 \mathrm{~L} / \mathrm{min}$ ) and no differences were found between treatment groups.

The respiratory quotient significantly increased during exercise in both the treatment groups, on Day 1 and Day 57 (Figure 3). The respiratory quotient was comparable between treatments at baseline and on Day 57 .

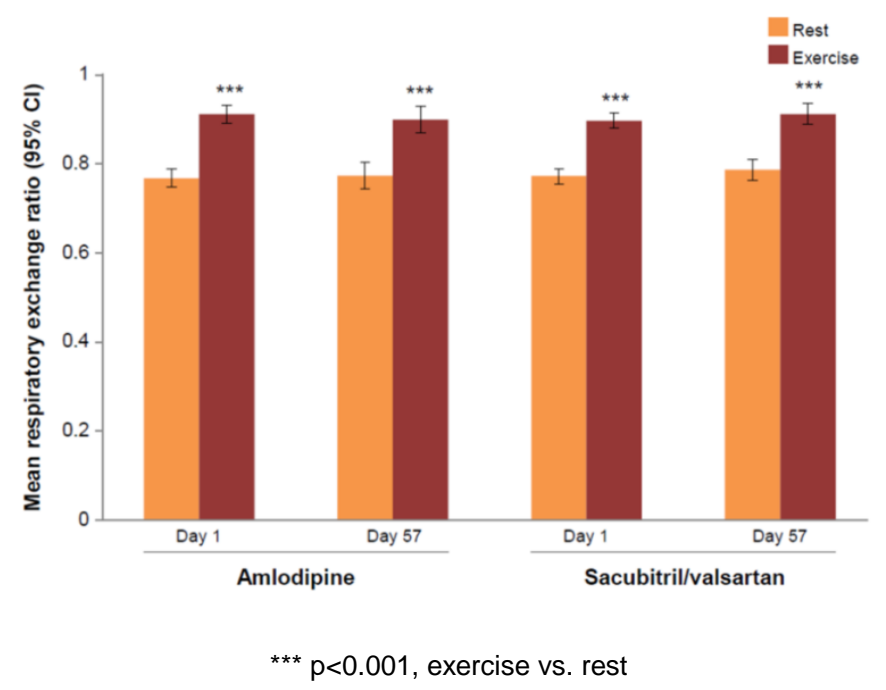

Figure 3. Oxidative metabolism-comparison of respiratory quotient between resting and exercise status- carbon dioxide to oxygen ratio.

Error bars indicate $95 \% \mathrm{Cl}$

\section{Plasma catecholamine concentrations}

When compared with resting levels, adrenaline concentrations increased significantly during exercise at all time-points in both treatment groups on Day 1 and 57 (Figure 4A). Compared with baseline, a significant reduction in adrenaline concentrations were observed on Day 57 in the amlodipine groups at all timepoints while the decrease was not statistically significant in the sacubitril/valsartan group. However, no significant differences were observed in the adrenaline concentrations between treatment groups at any time-point, except at 30 minutes $(p=0.012)$.

Plasma noradrenaline levels were significantly increased during exercise in both the treatment groups on Day 1 and Day $57(p<0.001)$ when compared with resting 
levels (Figure 4B). Noradrenaline levels increased incrementally during exercise on Day 1 and Day 57 in both the treatment groups, with no significant differences between treatments.

A

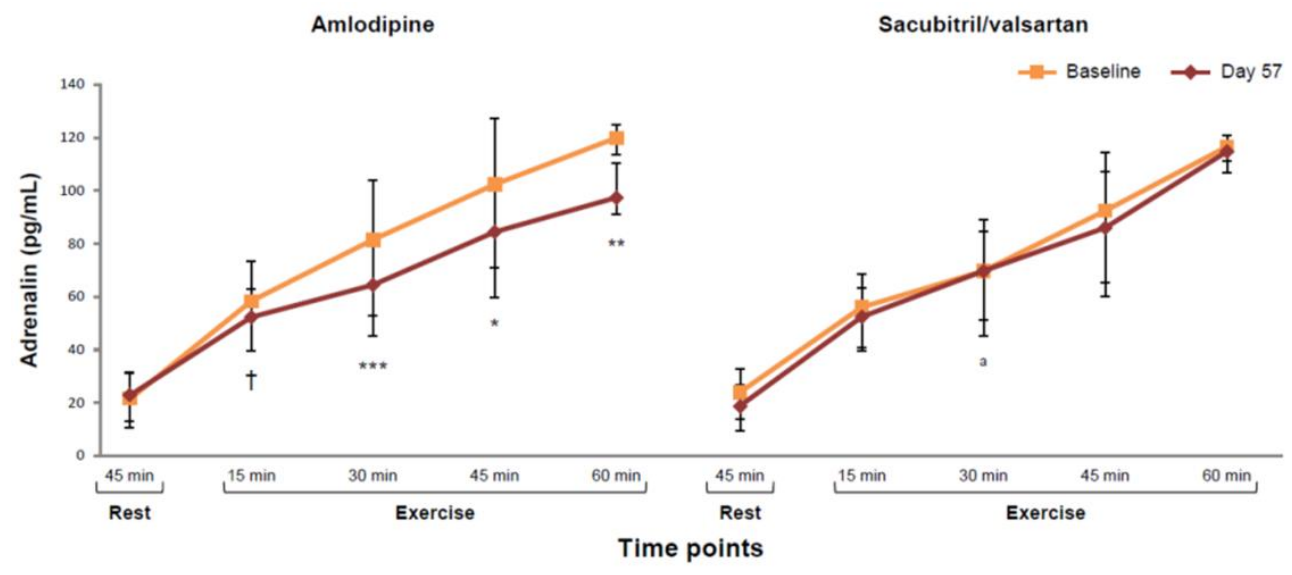

$\dagger p=0.044,{ }^{*} p=0.022,{ }^{* *} p=0.019,{ }^{* *} p<0.001$ vs. baseline. a $p=0.012$ vs. amlodipine

B

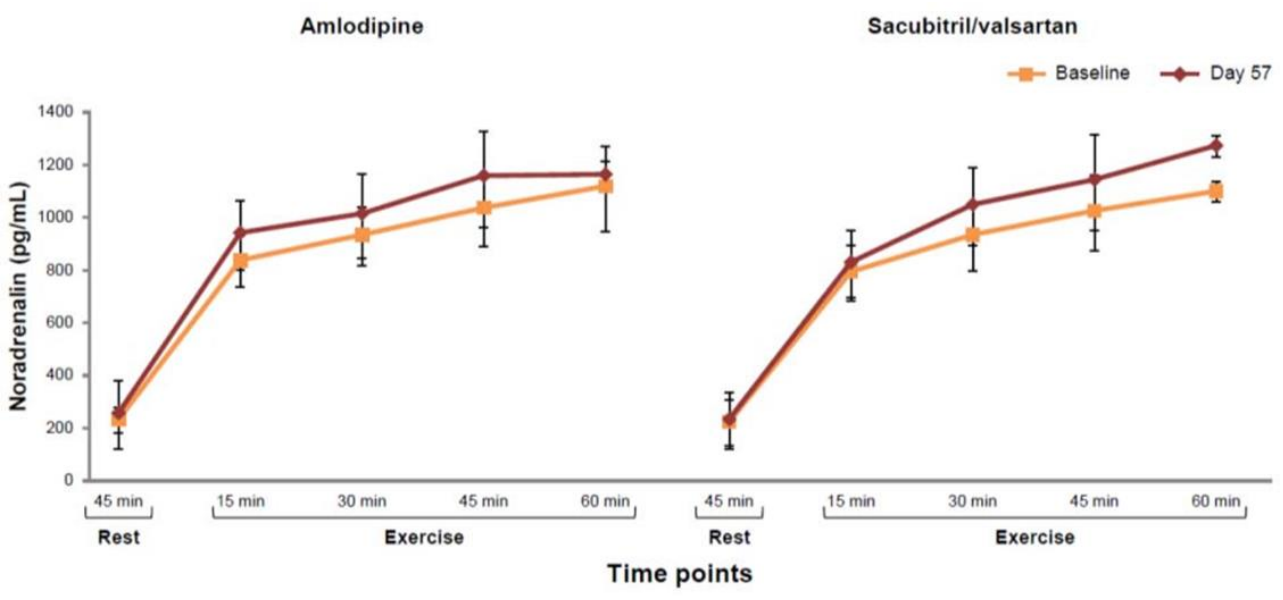

$p<0.001$ vs. rest

Figure 4. Analysis of plasma biomarkers during exercise: adrenaline $(\mathbf{A})$ and noradrenaline (B)

Error bars indicate $95 \% \mathrm{Cl}$. 
Blood pressure

After 8-weeks of treatment, systolic BP, diastolic BP and pulse pressure decreased from baseline in both treatment groups at rest. Systolic and diastolic BP and pulse rate values increased during exercise in both treatment groups on both Day 1 and Day 57 without clinically relevant differences between treatment groups (Table 1).

Table 1. Comparison of BP and pulse rate between treatments during exercise and rest

\begin{tabular}{|c|c|c|c|c|}
\hline Parameter & Day & Rest/Exercise & $\begin{array}{l}\text { LCZ696 (400 mg } \\
\text { QD) N=50 }\end{array}$ & $\begin{array}{c}\text { Amlodipine } \\
\text { (10 mg QD) } \\
\mathrm{N}=48\end{array}$ \\
\hline \multirow{4}{*}{$\mathrm{SBP}(\mathrm{mmHg})$} & \multirow{2}{*}{ Day 1} & Rest & $137.9 \pm 14.7$ & $135.8 \pm 12.8$ \\
\hline & & Exercise & $157.5 \pm 23.8$ & $146.5 \pm 23.3$ \\
\hline & \multirow{2}{*}{ Day 57} & Rest & $119.9 \pm 14.3$ & $125.9 \pm 10.2$ \\
\hline & & Exercise & $146.8 \pm 21.9$ & $137.6 \pm 20.5$ \\
\hline \multirow{4}{*}{$\mathrm{DBP}(\mathrm{mmHg})$} & \multirow{2}{*}{ Day 1} & Rest & $88.0 \pm 10.6$ & $86.8 \pm 8.9$ \\
\hline & & Exercise & $82.8 \pm 12.6$ & $84.3 \pm 12.4$ \\
\hline & \multirow{2}{*}{ Day 57} & Rest & $77.2 \pm 8.5$ & $81.2 \pm 7.2$ \\
\hline & & Exercise & $78.4 \pm 12.3$ & $78.8 \pm 11.9$ \\
\hline \multirow{4}{*}{$\begin{array}{l}\text { Pulse rate } \\
\text { (BPM) }\end{array}$} & \multirow{2}{*}{ Day1 } & Rest & $67.4 \pm 8.6$ & $66.7 \pm 9.3$ \\
\hline & & Exercise & $121.2 \pm 15.4$ & $111.4 \pm 25.6$ \\
\hline & \multirow{2}{*}{ Day 57} & Rest & $65.2 \pm 8.7$ & $69.2 \pm 10.5$ \\
\hline & & Exercise & $123.5 \pm 18.6$ & $115.6 \pm 16.5$ \\
\hline
\end{tabular}

Data are expressed as mean \pm SD.

SBP, systolic blood pressure; DBP, diastolic blood pressure, BPM, beats per minute 


\section{DISCUSSION}

The present study demonstrated that treatment with sacubitril/valsartan compared with amlodipine for 8 weeks did not elicit relevant changes in exercise-induced lipolysis and substrate oxidation in obese patients with hypertension. The exerciseinduced increase in abdominal subcutaneous adipose tissue and whole-body lipolysis was not augmented following sacubitril/valsartan treatment compared with amlodipine treatment. Moreover, the shift in substrate oxidation towards carbohydrate catabolism during exercise was comparable in both treatment groups, implying that sacubitril/valsartan did not significantly affect lipid utilization during acute exercise. We have previously observed significantly improved wholebody insulin sensitivity and a modest increase in resting abdominal subcutaneous adipose tissue lipolysis with no marked changes in whole-body lipolysis with sacubitril/valsartan compared with amlodipine treatment [11]. Overall, these findings imply that the beneficial cardiometabolic effects of sacubitril/valsartan may not be explained by changes in lipid mobilization or oxidation.

In this study, we used state-of-the-art methodology including $\left[1,1,2,3,3-{ }^{2} \mathrm{H}\right]$-glycerol tracer kinetics and abdominal subcutaneous adipose tissue microdialysis to assess whole-body and local lipolysis, respectively, in a large patient sample. Furthermore, we treated patients with a total daily dose of sacubitril/valsartan which provided superior BP control in patients with arterial hypertension (400 mg QD) [12] and reduced cardiovascular mortality and heart failure hospitalizations in patients with heart failure and reduced ejection fraction (HFrEF) (200 mg twice daily) compared with standard-of-care renin-angiotensin system (RAS) inhibition [7]. This study, therefore, was appropriately designed to study the effect of sacubitril/valsartan on lipid turnover.

Our study extends previous investigations on the role of neprilysin substrates and angiotensin II type-1 $\left(\mathrm{AT}_{1}\right)$-receptors in the regulation of lipid turnover. All components of the RAS are expressed in adipose tissue, and $\mathrm{AT}_{1}$-receptors have been implicated in the regulation of adipose tissue differentiation, inflammation, and metabolism [10]. Conflicting findings have been reported with respect to the effects of angiotensin II on adipose tissue lipolysis. More specifically, both increased [13,14] and decreased [15] subcutaneous adipose tissue lipolysis have been demonstrated [14]. Moreover, intravenous angiotensin II infusions and angiotensin converting enzyme inhibition did not elicit major changes in whole-body lipolysis as determined by glycerol tracer kinetics [16]. $\mathrm{AT}_{1}$-receptor blockade in human subjects did not increase lipolytic gene expression or lipolysis in abdominal subcutaneous adipose tissue $[17,18]$. However, long-term $\mathrm{AT}_{1}$-receptor blockade altered intramuscular lipid partitioning, manifested by decreased saturation of skeletal muscle triacylglycerol and diacylglycerol stores, reduced postprandial fatty acid spillover and lipolysis [19]. Overall, angiotensin II actions on AT ${ }_{1}$-receptors appear to have modest effects on lipid turnover. Although postprandial fatty acid handling has not been examined in this study, the present findings suggest that $\mathrm{AT}_{1}$-receptor blockade in the context of neprilysin inhibition by sacubitril/valsartan does not have clinically relevant effects on lipid mobilization or utilization.

Neprilysin degrades multiple peptides potentially modulating lipid metabolism such as natriuretic peptides (NPs), bradykinin, endothelin-1, and glucagon-like peptide 1 (GLP-1) [20]. We cannot discern contributions of individual neprilysin substrates to 
the observed metabolic response. While bradykinin has been suggested to attenuate lipolysis, endothelin-1 may increase lipolysis. However, endothelin-1 was significantly decreased following treatment of patients with HFrEF with sacubitril/valsartan for 21 days, and no changes in lipolysis for GLP-1 at high concentrations have been reported [21-25]. Among neprilysin substrates, lipolytic effects of NPs are particularly striking. NPs are more potent in stimulating human adipose tissue lipolysis than the prototypical $\beta$-adrenoreceptor agonist isoproterenol [26]. Since NP-induced lipolysis is observed only in primates, the utility of many preclinical animal models is limited [27]. NPs are released during physical exercise and have been suggested to provide lipid fuel to the working skeletal muscle. Excess NP-mediated lipid mobilization has been suggested as a potential limitation of therapeutic neprilysin inhibition. In fact, ex vivo lipolysis of subcutaneous adipose tissue was not desensitized in patients with heart failure despite increased circulating NP levels [28]. However, the lack of changes in exercise-induced lipolysis by sacubitril/valsartan observed in the present study is clinically reassuring.

Given the potent acute effect of NPs on human lipolysis, our findings are somewhat unexpected. Plasma noradrenaline and adrenaline increased to a similar extent at baseline and following treatment with amlodipine and sacubitril/valsartan, suggesting that opposing changes in sympatho-adrenal activity did not mask a direct treatment effect on lipolysis. The reduction in catecholamine concentrations observed with sacubitril/valsartan in this study is consistent with our previous observation [11]. Conflicting observations have been reported with respect to the effect of amlodipine therapy on noradrenaline concentrations [29-31] while valsartan treatment has been demonstrated to attenuate increases in plasma noradrenalin concentrations with larger reductions from baseline associated with lower risk of mortality and morbidity $[32,33]$. While sacubitril/valsartan improved insulin-mediated glucose disposal compared with amlodipine [11], potential antilipolytic effects of insulin in adipose tissue following sacubitril/valsartan have not been investigated before. We cannot completely rule out that improved insulin action in adipose tissue confounded our analysis. However, an alternative and more likely explanation is that NP actions in adipose tissue are not or to a lesser degree dependent on neprilysin activity. Indeed, a study in isolated human adipocytes suggests that clearance via the natriuretic peptide type- $C$ receptor, the so-called scavenger receptor, may be more important than neprilysin activity to control local NP availability [34]. Indeed, completely abolishing neprilysin activity using thiorphan did not modify atrial natriuretic peptide (ANP)-mediated lipolysis [34].

Noteworthy, we conducted our study in obese patients with hypertension. Given the differences in neurohormonal activity between patients with hypertension and heart failure, the extent to which our findings can be extrapolated to patients with HFrEF remains to be elucidated. However, a recent post-hoc analysis of the PARADIGM-HF trial showed that in patients with heart failure and type 2 diabetes mellitus, treatment with sacubitril/valsartan resulted in greater reductions in $\mathrm{HbA} 1_{\mathrm{c}}$ concentrations compared to those treated with enalapril. Moreover, sacubitril/valsartan treated patients with type 2 diabetes were less likely to require initiation of insulin treatment during the trial suggesting potential metabolic benefits of sacubitril/valsartan therapy in HF patients [35]. Finally, the selection of 
amlodipine as a metabolically neutral comparator does not allow distinguishing the contributions of neprilysin inhibition from those of $A T_{1}$-receptor blockade to the effects of sacubitril/valsartan observed in this study.

In conclusion, our study demonstrated that sacubitril/valsartan treatment did not result in clinically relevant changes in exercise-induced abdominal subcutaneous adipose tissue and whole-body lipolysis, energy expenditure and substrate oxidation compared with amlodipine. This finding is relevant because neprilysin substrates, particularly NPs, have been implicated in lipolysis and the pathogenesis of cardiac cachexia. Our findings further support the idea that neprilysin is of lesser importance in regulating NP availability in the vicinity of adipocytes. 


\section{REFERENCES}

1. Spriet LL. New insights into the interaction of carbohydrate and fat metabolism during exercise. Sports Med. 2014;44 Suppl 1:S87-96.

2. Bevilacqua S, Bonadonna R, Buzzigoli G, Boni C, Ciociaro D, Maccari F, Giorico MA, Ferrannini E. Acute elevation of free fatty acid levels leads to hepatic insulin resistance in obese subjects. Metabolism. 1987;36(5):502-6.

3. Roden M, Price TB, Perseghin G, Petersen KF, Rothman DL, Cline GW, Shulman GI. Mechanism of free fatty acid-induced insulin resistance in humans. J Clin Invest. 1996;97(12):2859-65.

4. Steinberg HO, Tarshoby M, Monestel R, Hook G, Cronin J, Johnson A, Bayazeed B, Baron AD. Elevated circulating free fatty acid levels impair endothelium-dependent vasodilation. J Clin Invest. 1997;100(5):1230-9.

5. Shulman Gl. Ectopic fat in insulin resistance, dyslipidemia, and cardiometabolic disease. N Engl J Med. 2014;371(23):2237-8.

6. Stinkens R, Goossens GH, Jocken JW, Blaak EE. Targeting fatty acid metabolism to improve glucose metabolism. Obes Rev. 2015;16(9):715-57.

7. McMurray JJ, Packer M, Desai AS, Gong J, Lefkowitz MP, Rizkala AR, Rouleau JL, Shi VC, Solomon SD, Swedberg K, Zile MR, Investigators P-H, Committees. Angiotensin-neprilysin inhibition versus enalapril in heart failure. $\mathrm{N}$ Engl $\mathrm{J}$ Med. 2014;371(11):993-1004.

8. Schling $\mathrm{P}$, Schafer $\mathrm{T}$. Human adipose tissue cells keep tight control on the angiotensin II levels in their vicinity. J Biol Chem. 2002;277(50):48066-75.

9. Moro C. Natriuretic peptides and fat metabolism. Curr Opin Clin Nutr Metab Care. 2013;16(6):645-9.

10. Goossens $\mathrm{GH}$. The renin-angiotensin system in the pathophysiology of type 2 diabetes. Obes Facts. 2012;5(4):611-24.

11. Jordan J, Stinkens R, Jax T, Engeli S, Blaak EE, May M, Havekes B, Schindler C, Albrecht D, Pal P, Heise T, Goossens GH, Langenickel TH. Improved Insulin Sensitivity With Angiotensin Receptor Neprilysin Inhibition in Individuals With Obesity and Hypertension. Clin Pharmacol Ther. 2017;101(2):254-63.

12. Kario K, Sun N, Chiang FT, Supasyndh O, Baek SH, Inubushi-Molessa A, Zhang Y, Gotou H, Lefkowitz M, Zhang J. Efficacy and safety of LCZ696, a first-in-class angiotensin receptor neprilysin inhibitor, in Asian patients with hypertension: a randomized, double-blind, placebo-controlled study. Hypertension. 2014;63(4):698705.

13. Boschmann M, Adams F, Schaller K, Franke G, Sharma AM, Klaus S, Luft FC, Jordan J. Hemodynamic and metabolic responses to interstitial angiotensin II in normal weight and obese men. J Hypertens. 2006;24(6):1165-71.

14. Boschmann M, Jordan J, Adams F, Christensen NJ, Tank J, Franke G, Stoffels M, Sharma AM, Luft FC, Klaus S. Tissue-specific response to interstitial angiotensin II in humans. Hypertension. 2003;41(1):37-41.

15. Goossens GH, Blaak EE, Saris WH, van Baak MA. Angiotensin II-induced effects on adipose and skeletal muscle tissue blood flow and lipolysis in normal-weight and obese subjects. J Clin Endocrinol Metab. 2004;89(6):2690-6.

16. Townsend RR. The effects of angiotensin-II on lipolysis in humans. Metabolism. 2001;50(4):468-72.

17. Goossens GH, Moors CC, van der Zijl NJ, Venteclef N, Alili R, Jocken JW, Essers $\mathrm{Y}$, Cleutjens JP, Clement K, Diamant M, Blaak EE. Valsartan improves adipose tissue function in humans with impaired glucose metabolism: a randomized placebocontrolled double-blind trial. PLoS One. 2012;7(6):e39930. 
18. Wang TJ, Larson MG, Keyes MJ, Levy D, Benjamin EJ, Vasan RS. Association of plasma natriuretic peptide levels with metabolic risk factors in ambulatory individuals. Circulation. 2007;115(11):1345-53.

19. Moors CC, Blaak EE, van der Zijl NJ, Diamant M, Goossens GH. The effects of longterm valsartan treatment on skeletal muscle fatty acid handling in humans with impaired glucose metabolism. J Clin Endocrinol Metab. 2013;98(5):E891-6.

20. Mangiafico S, Costello-Boerrigter LC, Andersen IA, Cataliotti A, Burnett JC, Jr. Neutral endopeptidase inhibition and the natriuretic peptide system: an evolving strategy in cardiovascular therapeutics. Eur Heart J. 2013;34(12):886-93c.

21. Kobalava Z, Kotovskaya Y, Averkov O, Pavlikova E, Moiseev V, Albrecht D, Chandra P, Ayalasomayajula S, Prescott MF, Pal P, Langenickel TH, Jordaan P, Rajman I. Pharmacodynamic and Pharmacokinetic Profiles of Sacubitril/Valsartan (LCZ696) in Patients with Heart Failure and Reduced Ejection Fraction. Cardiovasc Ther. 2016;34(4):191-8.

22. Eriksson AK, van Harmelen V, Stenson BM, Astrom G, Wahlen K, Laurencikiene J, Ryden M. Endothelin-1 stimulates human adipocyte lipolysis through the ET A receptor. Int J Obes (Lond). 2009;33(1):67-74.

23. Mori MA, Sales VM, Motta FL, Fonseca RG, Alenina N, Guadagnini D, Schadock I, Silva ED, Torres HA, dos Santos EL, Castro CH, D'Almeida V, Andreotti S, Campana AB, Sertie RA, Saad MJ, Lima FB, Bader M, Pesquero JB. Kinin B1 receptor in adipocytes regulates glucose tolerance and predisposition to obesity. PLoS One. 2012;7(9):e44782.

24. Bertin E, Arner P, Bolinder J, Hagstrom-Toft E. Action of glucagon and glucagon-like peptide-1-(7-36) amide on lipolysis in human subcutaneous adipose tissue and skeletal muscle in vivo. J Clin Endocrinol Metab. 2001;86(3):1229-34.

25. Villanueva-Penacarrillo ML, Marquez L, Gonzalez N, Diaz-Miguel M, Valverde I. Effect of GLP-1 on lipid metabolism in human adipocytes. Horm Metab Res. 2001;33(2):73-7.

26. Birkenfeld AL, Boschmann M, Moro C, Adams F, Heusser K, Franke G, Berlan M, Luft FC, Lafontan M, Jordan J. Lipid mobilization with physiological atrial natriuretic peptide concentrations in humans. J Clin Endocrinol Metab. 2005;90(6):3622-8.

27. Sengenes C, Zakaroff-Girard A, Moulin A, Berlan M, Bouloumie A, Lafontan M, Galitzky J. Natriuretic peptide-dependent lipolysis in fat cells is a primate specificity. Am J Physiol Regul Integr Comp Physiol. 2002;283(1):R257-65.

28. Birkenfeld AL, Adams F, Schroeder C, Engeli S, Jordan J. Metabolic actions could confound advantageous effects of combined angiotensin II receptor and neprilysin inhibition. Hypertension. 2011;57(2):e4-5.

29. Toal CB, Meredith PA, Elliott HL. Long-acting dihydropyridine calcium-channel blockers and sympathetic nervous system activity in hypertension: a literature review comparing amlodipine and nifedipine GITS. Blood Press. 2012;21 Suppl 1:3-10.

30. Stankovic S, Panz V, Klug E, Di Nicola G, Joffe BI. Amlodipine and physiological responses to brisk exercise in healthy subjects. Cardiovasc Drugs Ther. 1999;13(6):513-7.

31. de Champlain J, Karas M, Assouline L, Nadeau R, LeBlanc AR, Dube B, Larochelle P. Effects of valsartan or amlodipine alone or in combination on plasma catecholamine levels at rest and during standing in hypertensive patients. J Clin Hypertens (Greenwich). 2007;9(3):168-78.

32. Latini R, Masson S, Anand I, Judd D, Maggioni AP, Chiang YT, Bevilacqua M, Salio M, Cardano P, Dunselman PH, Holwerda NJ, Tognoni G, Cohn JN, Valsartan Heart Failure Trial I. Effects of valsartan on circulating brain natriuretic peptide and norepinephrine in symptomatic chronic heart failure: the Valsartan Heart Failure Trial (Val-HeFT). Circulation. 2002;106(19):2454-8. 
33. Anand IS, Fisher LD, Chiang YT, Latini R, Masson S, Maggioni AP, Glazer RD, Tognoni G, Cohn JN, Val-He FTI. Changes in brain natriuretic peptide and norepinephrine over time and mortality and morbidity in the Valsartan Heart Failure Trial (Val-HeFT). Circulation. 2003;107(9):1278-83.

34. Moro C, Klimcakova E, Lafontan M, Berlan M, Galitzky J. Phosphodiesterase-5A and neutral endopeptidase activities in human adipocytes do not control atrial natriuretic peptide-mediated lipolysis. Br J Pharmacol. 2007;152(7):1102-10.

35. Seferovic JP, Claggett B, Seidelmann SB, Seely EW, Packer M, Zile MR, Rouleau JL, Swedberg K, Lefkowitz M, Shi VC, Desai AS, McMurray JJV, Solomon SD. Effect of sacubitril/valsartan versus enalapril on glycaemic control in patients with heart failure and diabetes: a post-hoc analysis from the PARADIGM-HF trial. Lancet Diabetes Endocrinol. 2017;5(5):333-40. 
CHAPTER 5 


\section{The effects of angiotensin receptor neprilysin inhibition by sacubitril/valsartan on adipose tissue transcriptome and protein expression in obese hypertensive patients}

Stinkens R., van der Kolk B.W., Jordan J., Jax T., Engeli S., Heise T., Jocken J.W., May M., Schindler C., Havekes B., Schaper N., Albrecht D., Kaiser S., Hartmann N., Letzkus M., Langenickel T.H., Goossens G.H., Blaak E.E.

Submitted 


\begin{abstract}
Increased activation of the renin-angiotensin system is involved in the onset and progression of cardiometabolic diseases, while natriuretic peptides (NP) may exert protective effects. We have recently demonstrated that sacubitril/valsartan (LCZ696), a first-in-class angiotensin receptor neprilysin inhibitor, which blocks the angiotensin II type-1 receptor and augments natriuretic peptide levels, improved peripheral insulin sensitivity in obese hypertensive patients. Here, we investigated the effects of sacubitril/valsartan (400mg QD) treatment for 8 weeks on the abdominal subcutaneous adipose tissue (AT) phenotype compared to the metabolically neutral comparator amlodipine (10mg QD) in 70 obese hypertensive patients. Abdominal subcutaneous AT biopsies were collected before and after intervention to determine the AT transcriptome and expression of proteins involved in lipolysis, NP signaling and mitochondrial oxidative metabolism. Both sacubitril/valsartan and amlodipine treatment did not significantly induce AT transcriptional changes in pathways related to lipolysis, NP signaling and oxidative metabolism. Furthermore, protein expression of adipose triglyceride lipase (ATGL) $\left(P_{\text {time* }}\right.$ group $\left.=0.195\right)$, hormone-sensitive lipase $(H S L)\left(P_{\text {time* }}\right.$ group $\left.=0.458\right), H S L$-ser660

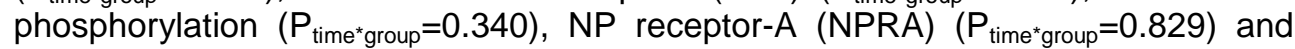
OXPHOS complexes $\left(P_{\text {time }}\right.$ group $\left.=0.964\right)$ remained unchanged. In conclusion, sacubitril/valsartan treatment for 8 weeks did not alter the abdominal subcutaneous AT transcriptome and expression of proteins involved in lipolysis, NP signaling and oxidative metabolism in obese hypertensive patients.
\end{abstract}




\section{INTRODUCTION}

Obesity is strongly associated with cardiometabolic risk factors [1], which is reflected by an increased risk for arterial hypertension, heart failure and type 2 diabetes mellitus (T2DM) [2]. An impaired adipose tissue function and excessive fat mass in obesity represent key factors in the development of insulin resistance and related chronic diseases, including cardiovascular disease and T2DM [3]. Evidence suggests that impaired insulin sensitivity in obesity might be related to an altered renin-angiotensin system (RAS) and natriuretic peptide (NP) signaling in adipose tissue. Blockade of the RAS using angiotensin-converting enzyme (ACE) inhibitors or angiotensin type-1 receptor blockers (ARB) has been shown to improve insulin sensitivity and beta-cell function [4] and reduces the incidence of T2DM [5] as reviewed elsewhere [6]. However, results are not consistent [7]. In addition, NPs are positively associated with insulin sensitivity and low atrial natriuretic peptide (ANP) concentrations are associated with an increased risk of developing arterial hypertension and T2DM [8]. In accordance, neprilysin (NEP), which is involved in the degradation and inactivation of NP, is linked to insulin resistance, increased blood pressure and impaired lipid metabolism [9]. Therefore, combined RAS blockade and NEP inhibition might have synergistic beneficial effects on blood pressure and peripheral insulin sensitivity. We recently demonstrated that combined ARB and NEP inhibition, using sacubitril/valsartan (LCZ696), improved peripheral insulin sensitivity following 8 weeks of treatment compared to amlodipine (AMLO) in obese hypertensive patients [10]. However, the mechanisms underlying these beneficial effects remain to be established.

Evidence suggests that both the RAS and ANP affect adipose tissue metabolism, thereby determining insulin sensitivity $[6,11]$. It has been shown that valsartan (ARB) reduced adipocyte size, increased adipose tissue blood flow and decreased gene expression of angiogenesis, adipogenesis and macrophage infiltration markers [12], which may have contributed to the valsartan-induced increased insulin sensitivity [4]. Furthermore, angiotensin II inhibited lipolysis in vitro in mature human adipocytes [13], although conflicting findings on adipose tissue lipolysis in vivo in humans have been reported $[14,15]$. ANP has been shown to increase adipose tissue lipid mobilization and oxidation [11] and we and others have recently demonstrated that ANP-mediated lipolysis is impaired in subcutaneous mature adipocytes from obese men with and without T2DM $[16,17]$.

Therefore, it is hypothesized that ARB and NEP inhibition with sacubitril/valsartan may affect adipose tissue function, thereby contributing to the observed improved peripheral insulin sensitivity in obese individuals [10]. The present study investigated the effects of sacubitril/valsartan compared to amlodipine treatment for 8 weeks on the abdominal subcutaneous adipose tissue transcriptome and protein expression profiles in obese hypertensive individuals. 


\section{METHODS}

\section{Study design}

Ninety-eight obese hypertensive patients participated in a multicenter, randomized, double-blind, parallel-group study to investigate the effects of sacubitril/valsartan (400mg QD) compared with amlodipine (10mg QD) treatment for 8 weeks. A detailed description of the inclusion and exclusion criteria is described elsewhere (clinicaltrials.gov - NCT01631864). Before and after treatment, abdominal subcutaneous adipose tissue biopsies were collected by needle aspiration under local anesthesia after an overnight fast. We determined adipose tissue gene expression profiles using microarray analysis in a subgroup of 70 patients who had a RNA Integrity Number (RIN) $\geq 5.0$, as described in detail below. Next, in a subgroup $(N=12-13)$, we determined the expression of proteins involved in the lipolytic pathway, the natriuretic peptide signaling pathway and mitochondrial oxidative phosphorylation. Subjects showing a treatment-induced increase in adipose tissue lipolysis in vivo following sacubitril/valsartan and unchanged lipolysis after amlodipine treatment were included in the latter analyses.

All patients gave written informed consent before participation. The Independent Ethical Committee or Institutional Review Board of each center reviewed the study protocol. The study was performed in accordance with the Declaration of Helsinki.

\section{Adipose tissue transcriptomics}

First, the integrity of abdominal subcutaneous adipose tissue total RNA was determined using the sample RNA Integrity Number (RIN), generated using a Agilent 2100 Bioanalyzer (Agilent Technologies Inc., Foster City, CA, USA). RIN values $<5.0$ indicated high level of sample total RNA degradation and were excluded from analyses [18]. Biopsies of 70 patients ( $N$ sacubitril/valsartan=36, $N$ amlodipine $=34$ ) were eligible for transcriptional analyses (RIN $\geq 5.0)$.

Whole-transcriptome analysis was carried-out using Affymetrix HG-U133plus2.0 oligonucleotide microarrays (Affymetrix Inc., Santa Clara, CA, USA) and the microarray files were pre-processed using the Robust Multi-array Average algorithm (RMA). The Chip Definition File (CDF) used for the RMA procedure corresponded to the public domain Michigan University Entrez CDF version 17.0. Transcripts showing median expression higher than 6 (log2-value) were combined and considered for statistical analyses. Evaluations comprised unsupervised (hypothesis-free) and supervised (targeted) assessments of which the latter explored the longitudinal treatment-induced effects on specific transcripts linked to lipolysis, fatty acid oxidation, mitochondrial biogenesis and adipokines.

\section{Adipose tissue protein expression}

A detailed description of the analysis can be found in the Supplementary Material. Briefly, abdominal subcutaneous adipose tissue was ground to a fine powder under liquid nitrogen and homogenized in RIPA buffer. The homogenate was lysed, vortexed and centrifuged and the supernatant was collected and stored at $-80^{\circ} \mathrm{C}$. The protein concentration was determined by the Bradford-based protein assay. Next, solubilized proteins $(15 \mu \mathrm{g})$ were separated on a precast gel and transferred 
onto a nitrocellulose membrane and quantitative Western Blot analysis was performed to determine the levels of proteins involved in the lipolytic pathway (adipose triglyceride lipase (ATGL), hormone sensitive lipase (HSL) and HSL serine 660 phosphorylation), the natriuretic peptide signaling pathway (natriuretic peptide receptor A (NPRA)) and mitochondrial oxidative phosphorylation (OXPHOS).

\section{Statistics}

Statistical analyses of gene expression data was performed with $R$ v.3.2.2 ( $R$ Development Core Team, Vienna, http://www.R-project.org) and the Bioconductor limma $R$ package v.3.26.8. Statistical significance was set at nominal $P \leq 0.05$ and P-values were corrected for multiple testing using the Bonferroni method. A total of 8319 transcripts (out of assessed 18898 transcripts/microarray) fulfilled the expression level filtering criteria and the Bonferroni adjusted threshold was therefore defined as $\mathrm{P}<6.01 \cdot 10^{-6}$. Longitudinal treatment-induced transcriptional changes were expressed as Ratio Change from baseline (RC; (post-treatment level).(pre-treatment level $\left.)^{-1}\right)$. Thresholds for minimum relevant treatment-induced effects were defined as $0.66 \geq R C \geq 1.50$ (i.e. $R C$ range equivalent to absolute fold changes $\geq 1.5$ ).

Protein expression data are expressed as mean \pm SEM. All variables were checked for normal distribution by Shapiro-Wilk test and variables were Ln-transformed to satisfy conditions of normality (HSL, HSL S660 phosphorylation, NPRA and OXPHOS). Data was analyzed using two-way repeated measures ANOVA, with time (pre, post) and treatment (sacubitril/valsartan, amlodipine) as factors. Bonferroni post-hoc correction was applied when a significant time*treatment interaction was found. Calculations were performed using SPSS v.21 for Mac OSX (IBM, Chicago, IL, USA) and $\mathrm{P} \leq 0.05$ was considered statistically significant.

\section{RESULTS}

\section{Subject characteristics}

Baseline characteristics of patients contributing to the present study are shown in Table 1. Importantly, the two groups were well matched and there were no major differences in clinical characteristics between groups.

\section{Transcriptomics}

At baseline, no significant differences in the abdominal subcutaneous adipose tissue transcriptome were found between treatment arms. Unsupervised assessments revealed 1443 longitudinally modulated transcripts (nominal $P<0.05$ ), but none of these fulfilled both thresholds for Bonferroni adjusted P-value and minimum treatment-induced RC effect. A set of selected transcripts is shown in Supplementary Table S1.

Supervised analyses identified no significant treatment-induced changes (defined based on the two thresholds stated above) in gene expression levels of transcripts involved in lipolysis, NP signaling, oxidative metabolism and adipokines (Figure 1). 
More specifically, gene expression of ATGL (PNPLA2), MGL (MGLL), lipoprotein lipase (LPL), perilipin-1 (PLIN1) and fatty acid binding protein 4 (FABP4) was not significantly altered by sacubitril/valsartan treatment (Figure 1). In addition, gene expression of natriuretic peptide receptors (NPR1, NPR3), adipokines (adiponectin $(A D I P O Q)$, leptin $(L E P))$ and oxidative metabolism markers (peroxisome proliferator-activated receptor gamma co-activator-related 1 (PPRC1), peroxisome proliferator-activated receptor gamma co-activator 1 alpha (PPARGC1A), nuclear respiratory factor 1 (NRF1), acyl-coenzyme A oxidase 1 (ACOX1) and uncoupling protein 2 (UCP2)) were not significantly affected (Figure 1).

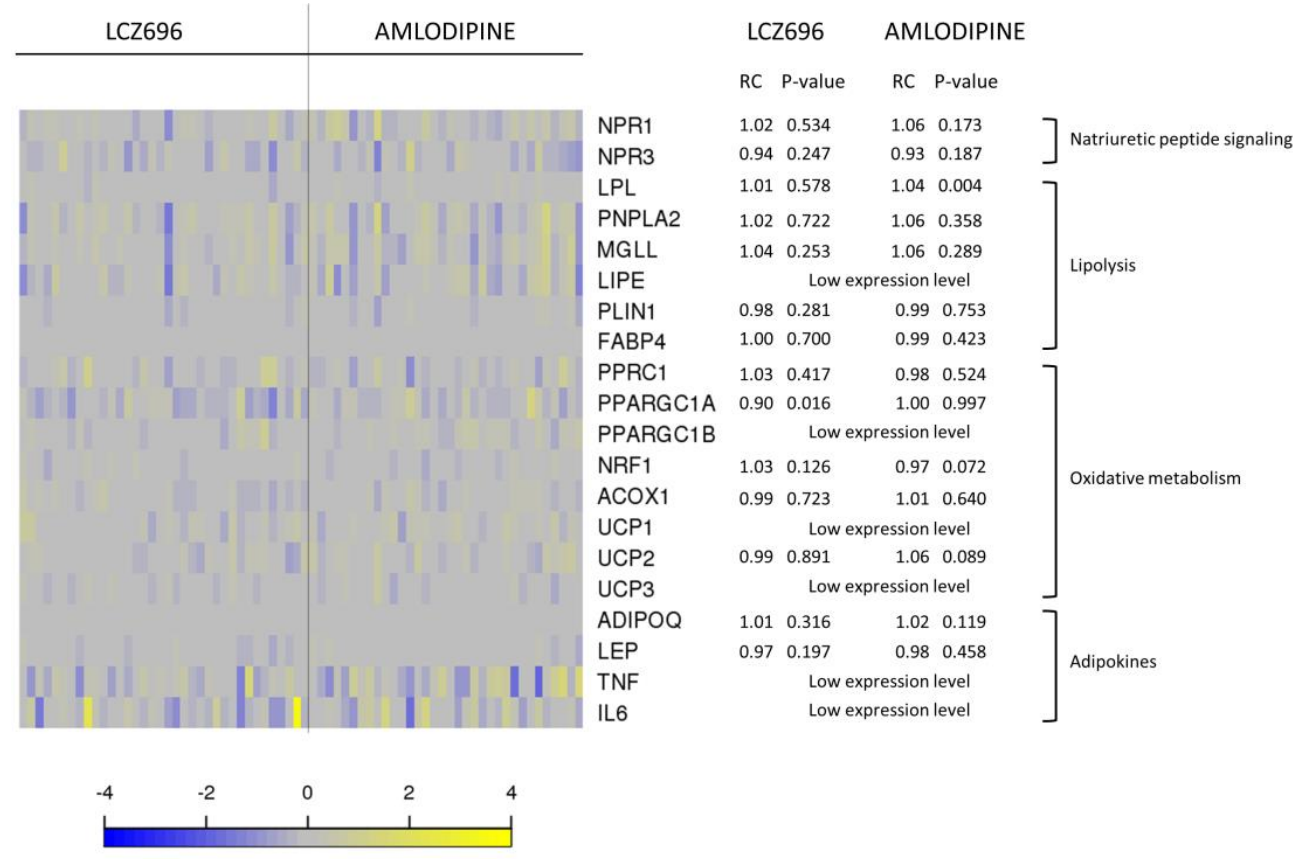

Figure 1. Targeted assessment of selected treatment-induced transcriptional changes in abdominal subcutaneous adipose tissue.

The heatmap plot shows per patient longitudinal treatment-induced gene expression changes [defined as $\log 2$ (post treatment expression) - log2(pre-treatment expression)] for selected transcripts related to natriuretic peptide signaling, lipolytic pathway, oxidative pathway and adipokines in abdominal subcutaneous adipose tissue ( $n=70$ patients; grouped per treatment arm). Color in the heatmap reflects transcript change from baseline per subject. Blue: downregulated transcripts; yellow: upregulated transcripts. The adjacent table depicts the corresponding transcript ratio change from baseline (RC) and P-value per treatment arm. 


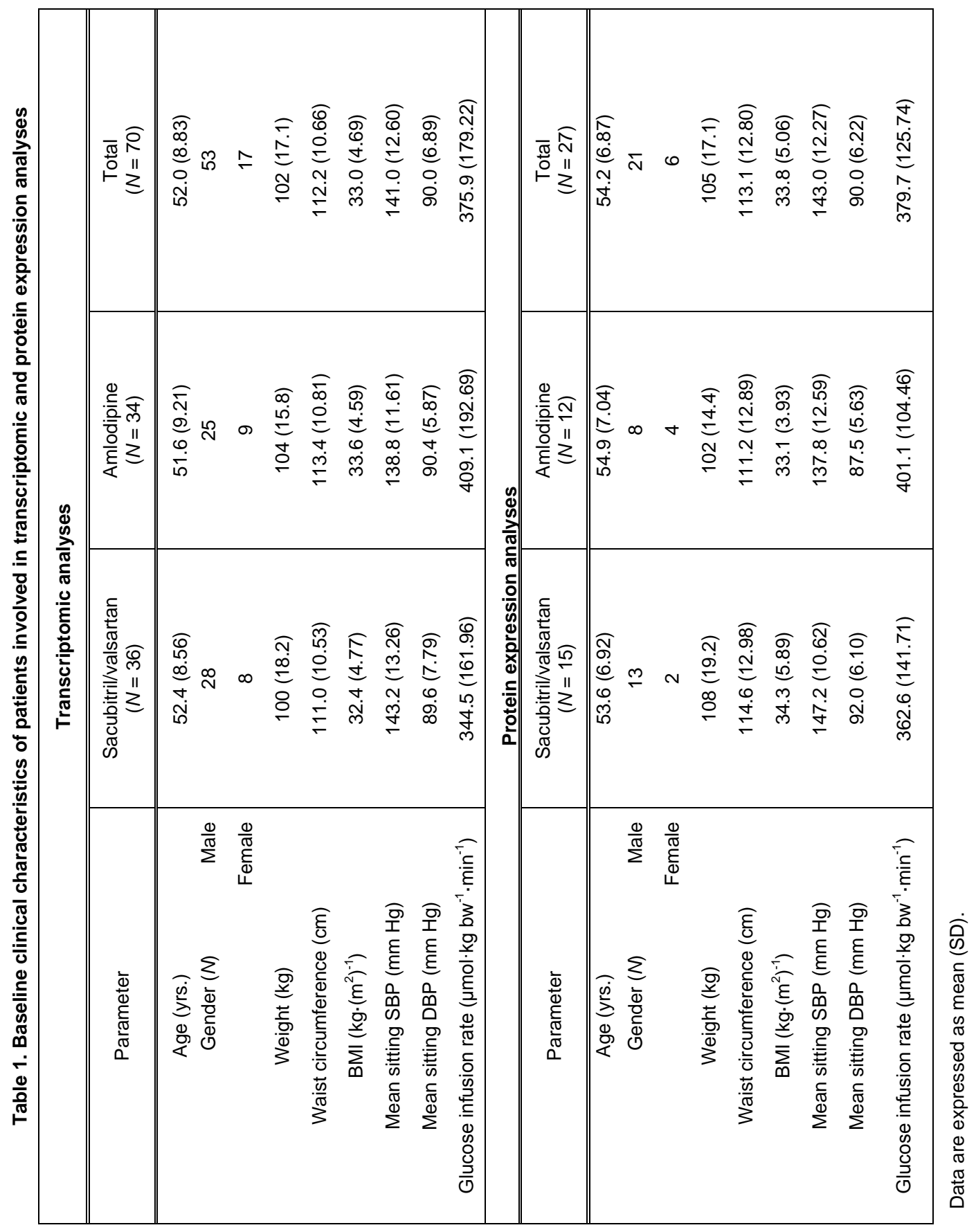


Protein expression

Sacubitril/valsartan treatment did not significantly change protein expression of ATGL (LCZ696: $1.00 \pm 0.21$ vs. $0.85 \pm 0.19$ AU; AMLO: $1.00 \pm 0.26$ vs. $1.36 \pm 0.30 \mathrm{AU}$; $P_{\text {time }}=0.583, P_{\text {timettreatment }}=0.195$; Figure $\left.2 A\right), H S L$ (LCZ696: $1.00 \pm 0.23$ vs. $1.38 \pm 0.24$ $\mathrm{AU}$; AMLO: $1.00 \pm 0.26$ vs. $1.00 \pm 0.18 \mathrm{AU} ; \mathrm{P}_{\text {time }}=0.141, \mathrm{P}_{\text {time }}{ }^{*}$ treatment $=0.458$; Figure 2B), HSL serine 660 phosphorylation (LCZ696: $1.00 \pm 0.16$ vs. $1.04 \pm 0.14 \mathrm{AU}$; AMLO: $1.00 \pm 0.25$ vs. $0.71 \pm 0.19 \mathrm{AU} ; \mathrm{P}_{\text {time }}=0.551, \mathrm{P}_{\text {timet }}{ }^{*}$ treatment $=0.340$; Figure $2 \mathrm{C}$ ) or NPRA (LCZ696: $1.00 \pm 0.24$ vs. $0.99 \pm 0.29$ AU; AMLO: $1.00 \pm 0.36$ vs. $0.96 \pm 0.30 \mathrm{AU}$; $\mathrm{P}_{\text {time }}=0.775, \mathrm{P}_{\text {timet }}{ }^{*}$ treatment $=0.829$; Figure 2D). Furthermore, total OXPHOS protein expression (LCZ696: $1.00 \pm 0.22$ vs. $1.76 \pm 0.48 \mathrm{AU}$; AMLO: $1.00 \pm 0.15$ vs. $1.74 \pm 0.50$ $\mathrm{AU} ; \mathrm{P}_{\text {time }}=0.125, \mathrm{P}_{\text {timetreatment }}=0.964$; Figure $2 \mathrm{E}$ ) remained unchanged following treatment. More specifically, OXPHOS complexes I-V were not affected (data not shown). Representative Western Blots are presented in Supplementary Figure S1. 
A

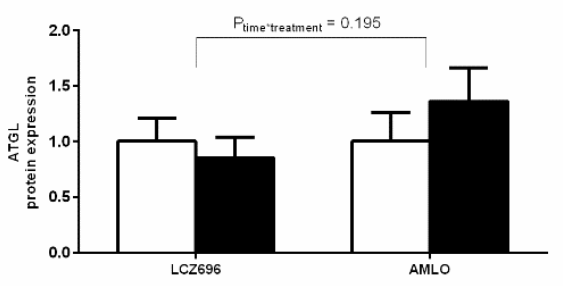

C

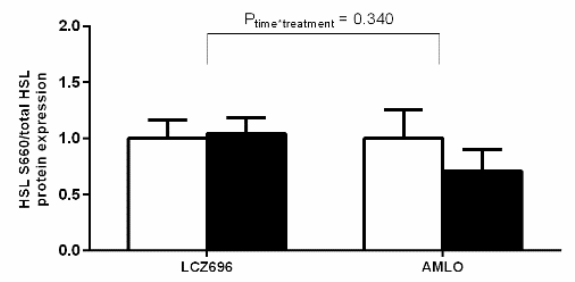

$E$

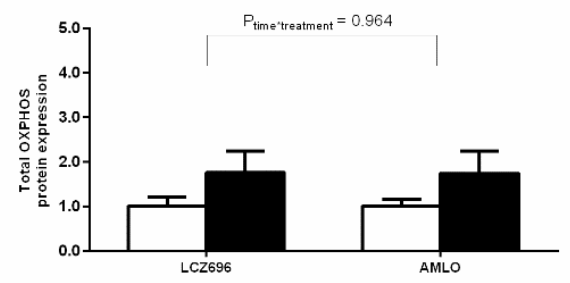

B

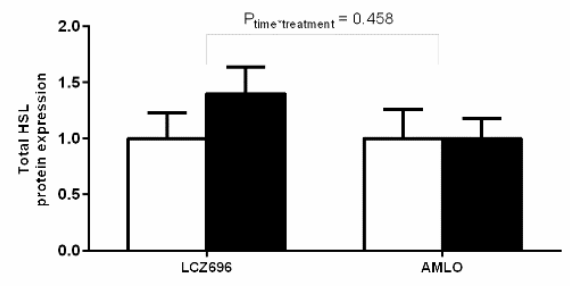

D

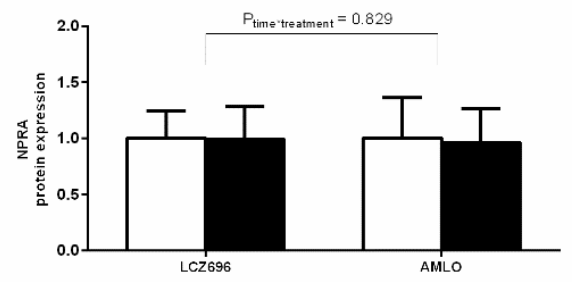

Figure 2. Protein expression in abdominal subcutaneous adipose tissue.

Expression of proteins involved in the lipolytic pathway (ATGL, N=12, A; HSL, N=13, B; HSL serine 660 phosphorylation, $N=13, \mathbf{C})$, the natriuretic peptide signaling pathway (NPR-A, $N=12, \mathbf{D})$ and mitochondrial oxidative metabolism (total OXPHOS, $N=13, \mathbf{E}$ ) expressed as fold change relative to baseline for each treatment arm (Sacubitril/valsartan: LCZ696 or Amlodipine: AMLO). Data are expressed as mean \pm SEM. White bars: baseline values; black bars: post-treatment values. Representative Western Blots are presented in Supplementary Figure S2. 


\section{DISCUSSION}

Here, we investigated the effects of sacubitril/valsartan versus amlodipine treatment for 8 weeks on the abdominal subcutaneous adipose tissue transcriptome and protein expression in obese hypertensive patients. We demonstrated that sacubitril/valsartan treatment did not significantly alter adipose tissue gene and protein expression of factors related to lipolysis, natriuretic peptide signaling and oxidative metabolism.

We recently demonstrated that in obese hypertensive patients sacubitril/valsartan treatment, which provides simultaneous ARB blockade and NEP inhibition, significantly increased peripheral insulin sensitivity [10]. Furthermore, sacubitril/valsartan slightly but significantly increased abdominal subcutaneous adipose tissue lipolysis, although no changes in whole-body lipolysis were observed [10]. Therefore, we hypothesized that RAS blockade and NEP inhibition has synergistic beneficial effects on abdominal subcutaneous adipose tissue metabolism and might underlie the observed improvement in insulin sensitivity. We showed that the abdominal adipose tissue phenotype was not significantly affected by 8 weeks of sacubitril/valsartan treatment in obese hypertensive patients. First, sacubitril/valsartan treatment did not reveal any transcriptional changes in abdominal subcutaneous adipose tissue, as determined by an unsupervised analysis. More specific, no treatment-induced changes in expression of genes involved in lipolysis, the NP signaling pathway and mitochondrial oxidative pathway were found. Secondly, the expression of proteins involved in these pathways, as well as post-translational modification of HSL, remained unchanged after the intervention.

Evidence suggests that both the RAS and NP system may affect adipose tissue metabolism, thereby contributing to improved insulin sensitivity [6, 11]. It has previously been shown that angiotensin II decreased adipose tissue lipolysis in vivo in humans [14, 19, 20] and in human isolated adipocytes [13], which seems to be mediated via the angiotensin II type-1 receptor. However, increased adipose tissue lipolysis has also been reported [15]. In accordance with our results, long-term ARB treatment with valsartan improved insulin sensitivity in subjects with impaired glucose metabolism [4], but adipose tissue gene and protein expression of several lipolytic enzymes remained unchanged [12].

Several studies have shown that ANP promotes adipose tissue lipid mobilization and oxidation in healthy individuals [11] via cGMP-mediated phosphorylation of HSL [21, 22]. Furthermore, ANP induced mitochondrial biogenesis and uncoupling in human adipocytes from healthy, non-diabetic women [23]. Here, we did not find significant changes in gene and protein expression of markers involved in lipolysis, phosphorylation of HSL and mitochondrial oxidative metabolism, which may be explained by reduced ANP-mediated signaling in the study population. Indeed, an impaired ANP-mediated lipolysis has recently been described in situ in subcutaneous adipose tissue and in vitro in subcutaneous adipocytes from obese individuals [16, 17]. Moreover, it has been shown that NPRC (NP clearance receptor) is increased in adipose tissue of obese hypertensive patients compared to lean and normotensive individuals [24], together with increased NEP expression in obesity [9]. These data suggest reduced NP signaling and increased NP clearance in adipose tissue in obesity. This may explain the unaltered 
subcutaneous adipose tissue metabolic phenotype following sacubitril/valsartan treatment in the present study.

Due to conservative multiple testing correction, we may have missed relevant treatment-induced changes in gene expression. However, the observed relative changes in gene expression were very modest, revealing only minor, potentially not physiologically-relevant, changes in gene expression.

\section{CONCLUSION}

The present study demonstrated that simultaneous RAS blockade and NEP inhibition following sacubitril/valsartan treatment for 8 weeks did not significantly alter the adipose tissue metabolic phenotype in obese hypertensive patients. More specifically, abdominal subcutaneous adipose tissue gene and protein expression of factors involved in lipolysis, natriuretic peptide signaling and mitochondrial oxidative metabolism remained unchanged. Therefore, alterations in the adipose tissue metabolic phenotype do not seem to contribute to the improved peripheral insulin sensitivity following 8 weeks of sacubitril/valsartan treatment [10]. 


\section{REFERENCES}

1. Reaven GM. Insulin resistance: the link between obesity and cardiovascular disease. Med Clin North Am. 2011;95(5):875-92.

2. Jordan J, Yumuk V, Schlaich M, Nilsson PM, Zahorska-Markiewicz B, Grassi G, Schmieder RE, Engeli S, Finer N. Joint statement of the European Association for the Study of Obesity and the European Society of Hypertension: obesity and difficult to treat arterial hypertension. J Hypertens. 2012;30(6):1047-55.

3. Stinkens R, Goossens GH, Jocken JW, Blaak EE. Targeting fatty acid metabolism to improve glucose metabolism. Obes Rev. 2015;16(9):715-57.

4. van der Zijl NJ, Moors CC, Goossens GH, Hermans MM, Blaak EE, Diamant M. Valsartan improves \{beta\}-cell function and insulin sensitivity in subjects with impaired glucose metabolism: a randomized controlled trial. Diabetes Care. $2011 ; 34(4): 845-51$.

5. McMurray JJ, Holman RR, Haffner SM, Bethel MA, Holzhauer B, Hua TA, Belenkov Y, Boolell M, Buse JB, Buckley BM, Chacra AR, Chiang FT, Charbonnel B, Chow CC, Davies MJ, Deedwania P, Diem P, Einhorn D, Fonseca V, Fulcher GR, Gaciong Z, Gaztambide S, Giles T, Horton E, Ilkova H, Jenssen T, Kahn SE, Krum H, Laakso M, Leiter LA, Levitt NS, Mareev V, Martinez F, Masson C, Mazzone T, Meaney E, Nesto R, Pan C, Prager R, Raptis SA, Rutten GE, Sandstroem H, Schaper F, Scheen A, Schmitz O, Sinay I, Soska V, Stender S, Tamas G, Tognoni G, Tuomilehto J, Villamil AS, Vozar J, Califf RM. Effect of valsartan on the incidence of diabetes and cardiovascular events. N Engl J Med. 2010;362(16):1477-90.

6. Goossens $\mathrm{GH}$. The renin-angiotensin system in the pathophysiology of type 2 diabetes. Obes Facts. 2012;5(4):611-24.

7. Bosch J, Yusuf S, Gerstein HC, Pogue J, Sheridan P, Dagenais G, Diaz R, Avezum A, Lanas F, Probstfield J, Fodor G, Holman RR. Effect of ramipril on the incidence of diabetes. N Engl J Med. 2006;355(15):1551-62.

8. Magnusson M, Jujic A, Hedblad B, Engstrom G, Persson M, Struck J, Morgenthaler NG, Nilsson P, Newton-Cheh C, Wang TJ, Melander O. Low plasma level of atrial natriuretic peptide predicts development of diabetes: the prospective Malmo Diet and Cancer study. J Clin Endocrinol Metab. 2012;97(2):638-45.

9. Standeven KF, Hess K, Carter AM, Rice GI, Cordell PA, Balmforth AJ, Lu B, Scott DJ, Turner AJ, Hooper NM, Grant PJ. Neprilysin, obesity and the metabolic syndrome. Int J Obes (Lond). 2011;35(8):1031-40.

10. Jordan J, Stinkens R, Jax T, Engeli S, Blaak EE, May M, Havekes B, Schindler C, Albrecht D, Pal P, Heise T, Goossens GH, Langenickel TH. Improved Insulin Sensitivity with Angiotensin Receptor Neprilysin Inhibition in Individuals with Obesity and Hypertension. Clin Pharmacol Ther. 2016.

11. Moro C. Natriuretic peptides and fat metabolism. Curr Opin Clin Nutr Metab Care. 2013;16(6):645-9.

12. Goossens GH, Moors CC, van der Zijl NJ, Venteclef N, Alili R, Jocken JW, Essers $\mathrm{Y}$, Cleutjens JP, Clement K, Diamant M, Blaak EE. Valsartan improves adipose tissue function in humans with impaired glucose metabolism: a randomized placebocontrolled double-blind trial. PLoS One. 2012;7(6):e39930.

13. Goossens GH, Blaak EE, Arner P, Saris WH, van Baak MA. Angiotensin II: a hormone that affects lipid metabolism in adipose tissue. Int $\mathrm{J}$ Obes (Lond). 2007;31(2):382-4.

14. Goossens GH, Blaak EE, Saris WH, van Baak MA. Angiotensin II-induced effects on adipose and skeletal muscle tissue blood flow and lipolysis in normal-weight and obese subjects. J Clin Endocrinol Metab. 2004;89(6):2690-6. 
15. Boschmann M, Jordan J, Adams F, Christensen NJ, Tank J, Franke G, Stoffels M, Sharma AM, Luft FC, Klaus S. Tissue-specific response to interstitial angiotensin II in humans. Hypertension. 2003;41(1):37-41.

16. Verboven K, Hansen D, Moro C, Eijnde BO, Hoebers N, Knol J, Bouckaert W, Dams A, Blaak EE, Jocken JW. Attenuated atrial natriuretic peptide-mediated lipolysis in subcutaneous adipocytes of obese type 2 diabetic men. Clin Sci (Lond). 2016;130(13):1105-14.

17. Ryden M, Backdahl J, Petrus P, Thorell A, Gao H, Coue M, Langin D, Moro C, Arner P. Impaired atrial natriuretic peptide-mediated lipolysis in obesity. Int $\mathrm{J}$ Obes (Lond). 2016;40(4):714-20.

18. Archer KJ, Mas VR, O'Brien TR, Pfeiffer R, Lum NL, Fisher RA. Quality assessment of microarray data in a multicenter study. Diagn Mol Pathol. 2009;18(1):34-43.

19. Boschmann M, Ringel J, Klaus S, Sharma AM. Metabolic and hemodynamic response of adipose tissue to angiotensin II. Obes Res. 2001;9(8):486-91.

20. Boschmann M, Rosenbaum M, Leibel RL, Segal KR. Metabolic and hemodynamic responses to exercise in subcutaneous adipose tissue and skeletal muscle. Int $\mathrm{J}$ Sports Med. 2002;23(8):537-43.

21. Moro C, Galitzky J, Sengenes C, Crampes F, Lafontan M, Berlan M. Functional and pharmacological characterization of the natriuretic peptide-dependent lipolytic pathway in human fat cells. J Pharmacol Exp Ther. 2004;308(3):984-92.

22. Sengenes C, Bouloumie A, Hauner H, Berlan M, Busse R, Lafontan M, Galitzky J. Involvement of a cGMP-dependent pathway in the natriuretic peptide-mediated hormone-sensitive lipase phosphorylation in human adipocytes. $\mathrm{J}$ Biol Chem. 2003;278(49):48617-26.

23. Bordicchia M, Liu D, Amri EZ, Ailhaud G, Dessi-Fulgheri P, Zhang C, Takahashi N, Sarzani R, Collins S. Cardiac natriuretic peptides act via p38 MAPK to induce the brown fat thermogenic program in mouse and human adipocytes. J Clin Invest. 2012;122(3):1022-36.

24. Dessi-Fulgheri P, Sarzani R, Tamburrini P, Moraca A, Espinosa E, Cola G, Giantomassi L, Rappelli A. Plasma atrial natriuretic peptide and natriuretic peptide receptor gene expression in adipose tissue of normotensive and hypertensive obese patients. J Hypertens. 1997;15(12 Pt 2):1695-9. 


\section{SUPPLEMENTARY MATERIAL}

\section{Protein expression analysis}

Adipose tissue ( $500 \mathrm{mg}$ ) was ground to a fine powder under liquid nitrogen and homogenized in radioimmunoprecipitation assay (RIPA) buffer (10 mM Tris (Calbiochem)-HCl (Merck, Darmstadt, Germany) buffered saline (Merck) with 0,1\% SDS (Bio-Rad Laboratories Inc, Hercules, CA, USA), 1\% Na-Deoxycholate (SigmaAldrich, St. Louis, MO, USA), 1\% NP-40 (Fluka) and a protease/phosphatase inhibitor cocktail (Cell Signaling Technology, Beverly, MA, USA). The homogenate was lysed on iced and vortexed for $5 \mathrm{~min}$ and centrifuged at 20,000 $\mathrm{g}$ for $30 \mathrm{~min}$ at $10^{\circ} \mathrm{C}$. The supernatant was carefully collected and aliquots were stored at $-80^{\circ} \mathrm{C}$. The protein concentration was determined by the Bradford-based protein assay (Santa Cruz Biotechnology, Dallas, TX, USA).

Next, solubilized proteins were separated on a precast gel (Criterion ${ }^{\mathrm{TM}}$ TGX any kD, Bio-Rad Laboratories Inc, Hercules, CA, USA) and transferred onto a nitrocellulose membrane (Trans Blot ${ }^{\circledR}$ Turbo $^{\mathrm{TM}}$ transfer system; Bio-Rad). Differences in loading were adjusted to total adipose tissue protein content (via Ponceau S (Sigma-Aldrich, St. Louis, MO, USA) staining), and appropriate positive controls (lysates of abdominal subcutaneous adipose tissue) were included.

Thereafter, quantitative Western Blot analysis was performed to determine the levels of ATGL (Cell Signaling Technology, Beverly, MA, USA), HSL (kind gift from Prof. Cecilia Holm, [Department of Cell and Molecular Biology, Lund University, Sweden]), HSL serine 660 phosphorylation (Cell Signaling Technology, Beverly, MA, USA) and NPRA (Abcam, Cambridge, MA, USA). The secondary antibody was a horseradish peroxidase (HRP) swine-anti-rabbit antibody (DakoCytomation, Glostrup, Denmark). Furthermore, OXPHOS blots were probed with Total OXPHOS Antibody Cocktail (Abcam, Cambridge, MA, USA) and a secondary HRPconjugated Rabbit-anti-Mouse antibody (DakoCytomation, Glostrup, Denmark). Antigen-antibody complexes were visualized using chemiluminescence by a ChemiDoc $^{\mathrm{TM}}$ XRS apparatus (Bio-Rad) and analyzed with Quantity One® software (Bio-Rad), which calculated the optical density units that are expressed as average intensity [average intensity = total intensity of the rows of pixels inside the band boundary divided by the number of rows, minus the background intensity]. 
SUPPLEMENTARY TABLE S1. Set of selected genes for the untargeted assessment of transcriptional changes in abdominal subcutaneous adipose tissue.

A total of 1443 transcripts were modulated by either sacubitril/valsartan $(n=916)$ or amlodipine $(n=668)$ treatment (nominal $\mathrm{P} \leq 0.05$, no multiple correction applied). A set of selected genes with corresponding ratio changes from baseline $(\mathrm{RC})$ and $\mathrm{P}$-value per treatment arm is shown.

\begin{tabular}{|c|c|c|c|c|c|}
\hline \multirow{2}{*}{$\begin{array}{c}\text { BIOLOGICAL } \\
\text { PATHWAY }\end{array}$} & \multirow{2}{*}{ GENES ID } & \multicolumn{2}{|c|}{ LCZ696 } & \multicolumn{2}{|c|}{ AMLO } \\
\hline & & $\mathrm{RC}$ & P-value & RC & P-value \\
\hline \multirow{45}{*}{ INFLAMMATION } & ALOX5AP & 0.76 & 0.001 & 0.93 & 0.483 \\
\hline & AOX 1 & 0.93 & 0.083 & 0.89 & 0.025 \\
\hline & BLNK & 0.91 & 0.040 & 0.96 & 0.430 \\
\hline & BTK & 0.88 & 0.011 & 0.94 & 0.337 \\
\hline & C3AR1 & 0.90 & 0.072 & 0.88 & 0.024 \\
\hline & CCL16 & 1.14 & 0.009 & 1.01 & 0.838 \\
\hline & CCL18 & 1.24 & 0.040 & 0.97 & 0.781 \\
\hline & CD14 & 0.89 & 0.035 & 0.95 & 0.351 \\
\hline & CD163 & 0.94 & 0.222 & 0.91 & 0.027 \\
\hline & CD44 & 0.95 & 0.044 & 0.89 & 0.000 \\
\hline & CD74 & 0.98 & 0.530 & 0.94 & 0.028 \\
\hline & CLU & 0.96 & 0.036 & 0.98 & 0.372 \\
\hline & CTSS & 0.85 & 0.009 & 0.88 & 0.118 \\
\hline & CXCL9 & 1.20 & 0.042 & 1.15 & 0.134 \\
\hline & CYBB & 0.86 & 0.003 & 0.93 & 0.215 \\
\hline & E2F3 & 0.95 & 0.020 & 1.00 & 0.887 \\
\hline & FCER1G & 0.87 & 0.023 & 0.87 & 0.042 \\
\hline & FYB & 0.85 & 0.050 & 1.01 & 0.944 \\
\hline & FYN & 0.98 & 0.075 & 0.96 & 0.036 \\
\hline & GAB2 & 0.96 & 0.131 & 0.95 & 0.027 \\
\hline & GBP2 & 0.91 & 0.005 & 0.96 & 0.236 \\
\hline & HCLS1 & 0.90 & 0.022 & 0.98 & 0.687 \\
\hline & HLA-DMA & 0.99 & 0.660 & 0.93 & 0.033 \\
\hline & IFI30 & 0.89 & 0.153 & 0.77 & 0.000 \\
\hline & IFIT2 & 0.91 & 0.009 & 1.03 & 0.593 \\
\hline & IFNGR1 & 0.99 & 0.649 & 0.95 & 0.021 \\
\hline & IRF1 & 0.90 & 0.040 & 1.09 & 0.134 \\
\hline & ITGB2 & 0.85 & 0.031 & 0.82 & 0.020 \\
\hline & LCP1 & 0.85 & 0.016 & 0.82 & 0.019 \\
\hline & LYN & 0.87 & 0.009 & 0.98 & 0.823 \\
\hline & MAP2K1 & 1.03 & 0.048 & 1.04 & 0.119 \\
\hline & MAP2K4 & 0.94 & 0.004 & 0.98 & 0.414 \\
\hline & MAP3K7 & 0.97 & 0.044 & 0.93 & 0.004 \\
\hline & MAX & 0.96 & 0.041 & 0.98 & 0.514 \\
\hline & PAK2 & 0.97 & 0.023 & 0.99 & 0.447 \\
\hline & PIK3CB & 0.96 & 0.027 & 0.97 & 0.290 \\
\hline & PLA2G7 & 1.01 & 0.917 & 0.71 & 0.001 \\
\hline & PLCG1 & 1.07 & 0.010 & 0.99 & 0.748 \\
\hline & PPP3CC & 1.00 & 0.927 & 0.96 & 0.047 \\
\hline & PTPN6 & 0.88 & 0.042 & 0.98 & 0.779 \\
\hline & PTPRC & 0.83 & 0.017 & 1.00 & 0.989 \\
\hline & RAC2 & 0.85 & 0.034 & 0.96 & 0.652 \\
\hline & RIPK1 & 0.98 & 0.249 & 0.95 & 0.021 \\
\hline & STAT1 & 0.93 & 0.006 & 1.03 & 0.316 \\
\hline & SYK & 0.85 & 0.001 & 0.89 & 0.078 \\
\hline
\end{tabular}




\begin{tabular}{|c|c|c|c|c|c|}
\hline \multirow{2}{*}{$\begin{array}{l}\text { BIOLOGICAL } \\
\text { PATHWAY }\end{array}$} & \multirow{2}{*}{ GENES ID } & \multicolumn{2}{|c|}{ LCZ696 } & \multicolumn{2}{|c|}{ AMLO } \\
\hline & & $\mathrm{RC}$ & P-value & RC & P-value \\
\hline OXIDATIVE & COX11 & 1.05 & 0.001 & 1.03 & 0.128 \\
\hline \multirow[t]{15}{*}{ METABOLISM } & COX412 & 1.09 & 0.016 & 1.11 & 0.024 \\
\hline & COX7A2 & 1.01 & 0.312 & 1.03 & 0.049 \\
\hline & COX7A2L & 0.97 & 0.028 & 0.98 & 0.288 \\
\hline & IDH2 & 1.02 & 0.530 & 1.10 & 0.011 \\
\hline & NDUFA5 & 1.01 & 0.831 & 1.06 & 0.049 \\
\hline & NDUFB4 & 1.03 & 0.179 & 0.94 & 0.047 \\
\hline & NDUFS1 & 1.00 & 0.939 & 1.08 & 0.010 \\
\hline & PDP1 & 0.94 & 0.002 & 0.99 & 0.497 \\
\hline & PPARGC1A & 0.89 & 0.016 & 1.00 & 0.997 \\
\hline & SLC25A11 & 1.01 & 0.804 & 1.10 & 0.009 \\
\hline & SLC25A16 & 0.99 & 0.820 & 1.10 & 0.019 \\
\hline & SLC25A18 & 1.08 & 0.041 & 1.07 & 0.117 \\
\hline & SLC25A27 & 0.99 & 0.862 & 0.92 & 0.046 \\
\hline & SLC25A33 & 1.04 & 0.337 & 1.10 & 0.038 \\
\hline & SUCLG1 & 0.96 & 0.017 & 1.02 & 0.417 \\
\hline NATRIURETIC & ADM & 0.95 & 0.027 & 0.95 & 0.071 \\
\hline PEPTIDE & EDN1 & 1.00 & 0.958 & 0.82 & 0.006 \\
\hline \multirow[t]{8}{*}{ SIGNALING } & FAP & 1.27 & 0.000 & 1.05 & 0.361 \\
\hline & GUCY1A2 & 1.12 & 0.000 & 1.11 & 0.001 \\
\hline & GUCY1A3 & 1.14 & 0.000 & 1.07 & 0.047 \\
\hline & GUCY1B3 & 1.12 & 0.000 & 1.04 & 0.150 \\
\hline & MME & 1.05 & 0.177 & 1.11 & 0.001 \\
\hline & PDE3A & 1.06 & 0.048 & 1.04 & 0.295 \\
\hline & PDE9A & 1.09 & 0.016 & 0.99 & 0.797 \\
\hline & RAPGEF5 & 1.09 & 0.031 & 1.04 & 0.362 \\
\hline & ACACA & 1.06 & 0.153 & 1.14 & 0.008 \\
\hline \multirow[t]{10}{*}{ METABOLISM } & ADIPOR1 & 0.91 & 0.006 & 0.99 & 0.802 \\
\hline & ANG & 0.94 & 0.076 & 0.91 & 0.037 \\
\hline & CIDEA & 0.90 & 0.096 & 0.85 & 0.024 \\
\hline & ELOVL5 & 0.99 & 0.522 & 1.03 & 0.048 \\
\hline & ELOVL6 & 1.09 & 0.431 & 1.42 & 0.003 \\
\hline & ELOVL7 & 0.93 & 0.095 & 0.90 & 0.047 \\
\hline & GPAM & 1.01 & 0.498 & 1.08 & 0.002 \\
\hline & LPL & 1.01 & 0.578 & 1.04 & 0.004 \\
\hline & PPARGC1A & 1.08 & 0.016 & 0.93 & 0.997 \\
\hline & PLA2G4C & 0.89 & 0.043 & 1.00 & 0.096 \\
\hline
\end{tabular}


A

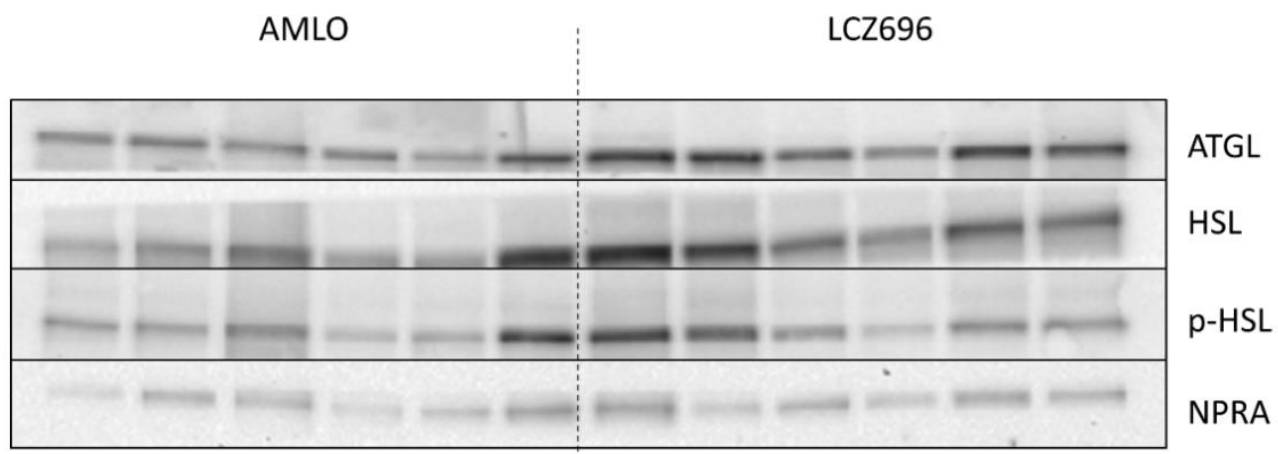

\begin{tabular}{ll:llll} 
PRE POST PRE POST PRE POST PRE POST PRE POST PRE POST \\
\hline
\end{tabular}

$\overline{\text { Patient A }} \overline{\text { Patient B }} \overline{\text { Patient C }} \overline{\text { Patient } D} \overline{\text { Patient } E} \overline{\text { Patient } F}$

B

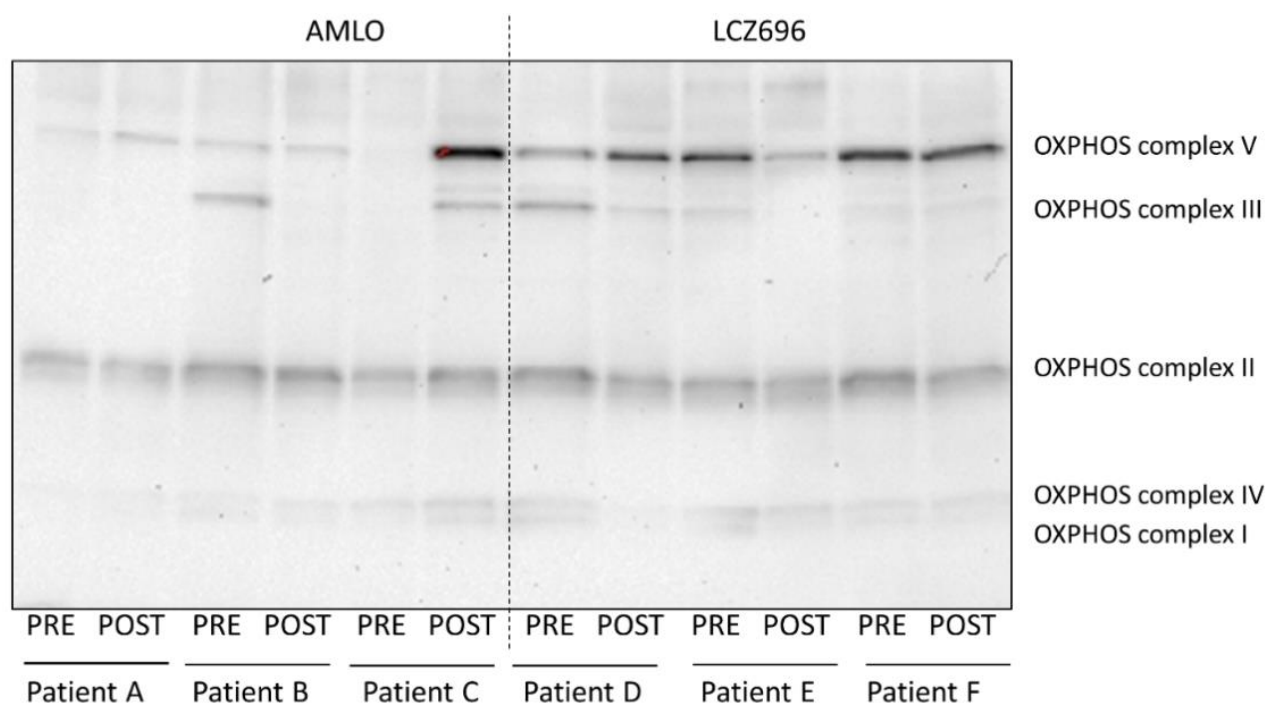

SUPPLEMENTARY FIGURE S2. Representative Western Blots for proteins involved in the lipolytic pathway, the natriuretic peptide signaling pathway and mitochondrial oxidative metabolism.

Membranes were probed with antibodies directed against total ATGL, total HSL, phosphorylated HSL (p-HSL) on Ser660, NPRA (A) and OXPHOS protein expression (B). 
CHAPTER 6 


\section{Exercise training-induced effects on the abdominal subcutaneous adipose tissue phenotype in obese humans}

Stinkens R. *, Brouwers B. *, Jocken J.W., Blaak E.E.,

Theunissen-Beekman K.F., Hesselink M. K., van Baak M., Schrauwen P., Goossens G.H.

* Shared authorship

To be submitted 


\section{ABSTRACT}

Aims/hypothesis: Rodent studies have indicated that physical exercise may improve adipose tissue (AT) function. We investigated the effects of a 12-weeks supervised, progressive exercise training program on adipocyte morphology and abdominal subcutaneous AT function in well-phenotyped, obese subjects.

Methods: 21 obese men (14 metabolically compromised - non-alcoholic fatty liver and/or type 2 diabetes - and 7 metabolically healthy controls) participated in a 12weeks supervised, progressive, combined exercise training program. At baseline and after intervention, abdominal subcutaneous AT biopsies were collected to determine 1) adipocyte morphology, 2) gene expression of markers for lipolysis, inflammation, browning, adipokines and mitochondrial biogenesis/function, 3) protein expression of mitochondrial oxidative phosphorylation (OXPHOS) complexes and 4) ex vivo basal and $\beta_{2}$-adrenergic stimulated lipolysis.

Results: At baseline, AT gene expression of HSL $(P=0.005), C G I-58 \quad(P<0.001)$ and $P G C-1 \alpha(P=0.037)$ were significantly lower in the metabolically compromised as compared to metabolically healthy obese subjects. Mean adipocyte diameter and total OXPHOS protein content in AT were comparable between groups. The exercise training program, which increased maximal aerobic capacity $\left(P_{\text {time }}<0.001\right)$ and muscle strength $\left(P_{\text {time }}<0.001\right)$, slightly reduced AT mass $\left(\sim 0.7 \mathrm{~kg}, P_{\text {time }}=0.037\right)$ but did not affect abdominal subcutaneous adipocyte size $\left(P_{\text {time }}=0.860\right)$, AT gene expression of markers for mitochondrial biogenesis and function, browning, lipolysis, inflammation and adipokines, total OXPHOS protein content $\left(P_{\text {time }}=0.826\right)$ and $\beta_{2}$-adrenergic sensitivity of lipolysis $\left(P_{\text {time }}=0.555\right)$, irrespective of baseline metabolic status.

Conclusions/interpretation: A 12-weeks supervised, progressive exercise training program did neither alter abdominal subcutaneous adipocyte morphology and AT gene and protein expression of markers related to adipose tissue function, nor $\beta_{2}$-adrenergic sensitivity of lipolysis in obese subjects, irrespective of baseline metabolic status. 


\section{INTRODUCTION}

The obesity epidemic is paralleled by a tremendous increase in the prevalence of obesity-related diseases, including type 2 diabetes (T2DM), non-alcoholic fatty liver (NAFL), cardiovascular disease and certain types of cancer [1]. A sedentary lifestyle is a major contributor to obesity and related complications. In line, increased habitual physical activity and exercise training may have beneficial effects on insulin sensitivity and glucose homeostasis in obese, insulin resistant and T2DM patients [2-4]. Therefore, increasing physical activity is a recommended lifestyle modification in the prevention and treatment of obesity-related disorders, including T2DM [5].

Since skeletal muscle is responsible for the majority of glucose disposal, adaptations in skeletal muscle metabolism are thought to play a central role in the exercise training-induced improvement of insulin sensitivity. Adipose tissue dysfunction in obesity, however, represents a key step in the development of obesity-related insulin resistance and chronic diseases [6,7]. The reason for this is that adipocyte hypertrophy in obesity promotes low-grade inflammation and decreases the adipose tissue lipid buffering capacity. Consequently, lipids accumulate in non-adipose tissues (e.g. skeletal muscle and liver) when lipid supply exceeds fat oxidation, thereby accelerating the development and progression of insulin resistance and chronic metabolic diseases [6-8].

Interestingly, there is evidence that exercise training may improve white adipose tissue function [9]. Several rodent studies demonstrated that exercise training increased adipose tissue mitochondrial biogenesis [10, 11] and function [12-14], induced browning of white adipose tissue $[10,11,13,15,16]$ and altered adipokine expression [17, 18]. Furthermore, transplantation of white adipose tissue from trained animals to untrained recipients markedly improved skeletal muscle glucose uptake [13], suggesting that improvement of adipose tissue function may contribute to the increased peripheral insulin sensitivity after exercise training. However, human studies that investigated the effects of exercise training on the adipose tissue phenotype are scarce. Exercise training has been shown to increase gene expression of peroxisome proliferator-activated receptor-gamma coactivator-1 alpha (PGC-1a) [19] and oxidative metabolism markers [20], yet conflicting data regarding the expression of adipokines and markers of lipolysis in human adipose tissue have been reported [21-29]. Furthermore, the evidence that exercise training enhances adipose tissue lipolysis, assessed either ex vivo in isolated adipocytes or in vivo at rest and in response to a lipolytic stimulus, is inconsistent and complicated by confounding factors such as recent energy balance, as reviewed [30]. Importantly, most human studies that have investigated the exercise traininginduced effects on adipose tissue metabolism did not perform detailed metabolic phenotyping. Therefore, it remains to be established whether the metabolic phenotype at baseline determines study outcomes.

The aim of the present study was to investigate the effects of a 12-weeks supervised, progressive, combined exercise training program on abdominal subcutaneous adipocyte morphology, adipose tissue gene expression of markers related to mitochondrial biogenesis/function, browning, lipolysis, inflammation and adipokines and protein expression of mitochondrial oxidative phosphorylation (OXPHOS) in obese, metabolically healthy and metabolically compromised 
individuals, matched for age and BMI. Furthermore, using isolated adipocytes from these subjects, we determined the exercise training-induced effects on ex vivo basal and $\beta_{2}$-adrenergic stimulation of lipolysis.

\section{METHODS}

\section{Study design}

Twenty-one sedentary, middle-aged (40-70 yrs), overweight/obese men (14 NAFL/T2DM and 7 age and BMI-matched metabolically healthy control subjects) participated in the present study, which was conducted within the framework of a larger clinical trial designed to primarily investigate the effects of exercise training on liver fat content, hepatic, adipose tissue and peripheral insulin sensitivity [31]. Control subjects $(n=7)$ had low liver fat content (all $\leq 4 \%$ ), as measured with proton magnetic resonance spectroscopy $\left({ }^{1} \mathrm{H}-\mathrm{MRS}\right)$, in the absence of liver dysfunction (defined as alanine aminotransferase (ALAT) >2.5 times normal values) and had to be normoglycemic according to the WHO criteria. Subjects were defined as NAFL patients $(n=7)$ when having a liver fat content $\geq 5 \%$ as measured with ${ }^{1} \mathrm{H}-\mathrm{MRS}$, in addition to a fasting plasma glucose concentration $<7.0 \mathrm{mmol} / \mathrm{l}$. Furthermore, at screening, T2DM patients $(n=7)$ were allowed to be on sulphonyl urea, metformin, dipeptidyl peptidase-4 inhibitors therapy (or a combination) for at least 6 months with stable dosage for at least 2 months or on a dietary treatment for 6 months, with fasting plasma glucose concentrations $\geq 7.0$ and $<10.0 \mathrm{mmol} / \mathrm{l}$. Liver fat content was not a selection criteria for T2DM patients. All subjects gave written informed consent before participation in the study. The Medical Ethical Committee of Maastricht University Medical Center ${ }^{+}$approved the study protocol, which was performed according the principles expressed in the Declaration of Helsinki.

All participants were asked not to change their habitual dietary intake during the study period. General exclusion criteria were unstable body weight, cardiovascular disease, impaired renal function, hemoglobin $<7.5 \mathrm{mmol} / \mathrm{l}$, blood pressure $>160 / 100 \mathrm{mmHg}$, participation in a weight-loss or exercise program, history of substantial alcohol use (>3 units/day), history of drug abuse, use of beta-blockers, anti-thrombotic medication, insulin therapy and use of medication known to interfere with glucose homeostasis (except for T2DM patients).

At screening, routine laboratory analyses and physical examinations were performed, medical history was checked and a resting electrocardiogram (ECG) was taken. Maximal power output $\left(\mathrm{W}_{\max }\right)$ and maximal aerobic capacity $\left(\mathrm{VO}_{2 \max }\right)$ were assessed during a graded cycling test with concurrent ECG until exhaustion. Body composition was determined using DEXA (Hologic Discovery A, Waltham, MA, USA). Furthermore, a two-step hyperinsulinemic-euglycemic clamp with primed D-[6,6- $\left.{ }^{2} \mathrm{H}_{2}\right]$-glucose was performed to assess peripheral, hepatic and adipose tissue insulin sensitivity, as described elsewhere [31]. 


\section{Exercise training protocol}

Subjects participated in a 12-weeks supervised, progressive exercise training program. Aerobic exercise training was performed on a cycle ergometer twice a week for $30 \mathrm{~min}$ at $70 \% \mathrm{~W}_{\max }$, which was determined just before the start of the intervention. Resistance exercise training, which focused on large muscle groups (chest press, lat pull down, leg extension, shoulder press, horizontal row, leg press, triceps extensions and biceps curls), was performed once a week and comprised three series of ten repetitions at $60 \%$ of subjects' previously determined one repeated maximum (1RM). The $1 \mathrm{RM}$ test was preceded by a familiarization trial. Warming-up and cooling-down sessions of 5 min were performed on a stationary bike at $45 \% \mathrm{~W}_{\max }$. Every 4 weeks, $1 \mathrm{RM}$ and $\mathrm{VO}_{2 \max }$ were reassessed and training loads were adjusted accordingly to assure that the training stimulus was maintained. At baseline and after the 12-weeks training program, several measurements were performed, as described below.

\section{Adipose tissue biopsies}

After an overnight fast, an abdominal subcutaneous adipose tissue biopsy ( $1 \mathrm{~g})$ was collected $6-8 \mathrm{~cm}$ lateral from the umbilicus, under local anesthesia $(2 \%$ lidocaine) by needle biopsy. The biopsy was washed with sterile saline and visible blood vessels were removed. One part of the biopsy was snap frozen in liquid nitrogen and stored at $-80^{\circ} \mathrm{C}$ for gene and protein expression analyses, whereas two parts were processed for determination of adipocyte morphology and measurement of ex vivo lipolysis, as described below.

\section{Adipocyte morphology}

A part of the adipose tissue biopsy was fixed overnight in $4 \%$ paraformaldehyde and embedded in paraffin for histological sections $(8 \mu \mathrm{m})$. Sections were cut from paraffin-embedded tissue, mounted on microscope glass slides and dried overnight in an incubator at $37^{\circ} \mathrm{C}$. The sections were stained with hematoxylin (VWR, Radnor, PA, USA) and eosin (Klinipath BV, Duiven, The Netherlands). Digital images were captured with a Leica DFC320 digital camera (Leica, Rijswijk, The Netherlands) at x20 magnification (Leica DM3000 microscope, Leica, Rijswijk, The Netherlands) and computerized morphometric analysis (Leica QWin V3, Cambridge, UK) of individual adipocytes was performed in a blinded manner. Approximately 400 adipocytes per sample were measured.

\section{Gene expression}

Total RNA was extracted from frozen adipose tissue biopsies ( $500 \mathrm{mg})$ using Trizol chloroform extraction (Invitrogen, Cergy Pontoise, France) and 300ng RNA was reversed transcribed using iScript cDNA synthesis kit (BIO-RAD). Gene expression for markers of lipolysis (ATGL [PNPLA2], HSL [LIPE], CGI-58 and PLIN1 [perilipin 1]), inflammation (TNFa, IL-6, MCP-1 [CCL2], CD68), browning (CIDEA, PRDM16), mitochondrial biogenesis (PGC-1a [PPARGC1A]) and adipokine expression (ADIPOQ and $L E P$ ) (supplementary table 1 for primer sequences) was determined in a total volume of $25 \mu \mathrm{L}$ containing $12.5 \mathrm{ng}$ cDNA using SYBR-Green based qPCR (iCycler/MylQ, BIO-RAD). Results were 
calculated via the $2^{-\Delta C T}$ method and normalized for $18 S$ (housekeeping gene) ribosomal RNA.

\section{Protein expression}

A detailed description can be found in the supplementary material. Briefly, subcutaneous adipose tissue $(\sim 500 \mathrm{mg})$ was ground to a fine powder under liquid nitrogen and homogenized in radioimmunoprecipitation assay buffer. The homogenate was lysed, vortexed and the supernatant was collected and stored at $80^{\circ} \mathrm{C}$. The protein concentration was determined by the Bradford-based protein assay (Santa Cruz Biotechnology, Dallas, TX, USA).

Next, solubilized proteins $(15 \mu \mathrm{g})$ were separated on a precast gel (Criterion ${ }^{\mathrm{TM}}$ TGX any kD, Bio-Rad Laboratories Inc, Hercules, CA, USA) and transferred onto a nitrocellulose membrane (Trans Blot ${ }^{\circledR}$ Turbo ${ }^{\text {TM }}$ transfer system; Bio-Rad).

Thereafter, quantitative Western Blot analysis was performed to determine the levels of OXPHOS proteins. OXPHOS blots were probed with Total OXPHOS Antibody Cocktail (Mitoscience/Abcam, Cambridge, MA, USA) and a secondary horseradish peroxidase (HRP)-conjugated Rabbit-anti-Mouse antibody (DakoCytomation, Glostrup, Denmark). Antigen-antibody complexes were visualized using chemiluminescence by a ChemiDoc ${ }^{\mathrm{TM}}$ XRS apparatus (Bio-Rad) and analyzed with Quantity One ${ }^{\circledR}$ software (Bio-Rad), which calculated the optical density units that are expressed as average intensity.

\section{Ex vivo adipocyte lipolysis}

Ex vivo adipocyte lipolysis was determined in 15 individuals and mature adipocytes were isolated from the subcutaneous adipose tissue following collagenase digestion. First, digestion was performed for $60 \mathrm{~min}$ at $37^{\circ} \mathrm{C}$ in a Krebs-Ringer phosphate buffer, containing $100 \mathrm{mg}$ glucose $/ 100 \mathrm{ml}$ and $4 \%$ bovine serum albumin with $2 \mathrm{mg} / \mathrm{ml}$ collagenase (Sigma-Aldrich, Zwijndrecht, The Netherlands). Secondly, adipocytes ( 5,000-10,000 cells/incubation) were incubated with or without increasing concentration of salbutamol (specific $\beta_{2}$-adrenergic receptor agonist) $\left(10^{-9}-10^{-4} \mathrm{M}\right.$; GlaxoSmithKline, Zeist, The Netherlands) for $2 \mathrm{~h}$ at $37^{\circ} \mathrm{C}$ in Krebs-Ringer phosphate buffer. Thereafter, incubation medium was collected and stored at $-80^{\circ} \mathrm{C}$ until analysis. Glycerol concentration in the medium, which is an indicator of complete TAG hydrolysis (lipolysis), was determined using the EAPL200 EnzyChrom Adipolysis Assay Kit (Glentaur Europe BVBA, Kampenhout, Belgium).

\section{Biochemistry}

Arterialized blood samples were collected and immediately centrifuged at $4^{\circ} \mathrm{C}$ for $10 \mathrm{~min}$ at $1000 \mathrm{~g}$ and plasma was snap frozen in liquid nitrogen and stored at $80^{\circ} \mathrm{C}$ until further analysis. Plasma non-esterified fatty acid (NEFA; Wako NEFA C test kit; Wako Chemicals, Neuss, Germany) and glucose (hexokinase method; LaRoche, Basel, Switzerland) concentrations were measured with enzymatic assays, whereas triacylglycerol (TAG) concentrations were measured colorimetrically (Roche, Vienna, Austria), automated on a Cobas Fara/Mira. Plasma insulin and serum liver function parameters (aspartate aminotransferase 
(ASAT), alanine aminotransferase (ALAT), $\mathrm{\gamma}$-glutamyl transpeptidase (GGT)) were routinely measured and analyzed at the clinical chemistry department in the hospital.

\section{Statistics}

Student's unpaired $t$-test was used for baseline comparisons between groups. The effects of exercise training in metabolically healthy and metabolically compromised obese subjects were compared by two-way repeated measures ANOVA, using time (baseline and post-intervention) as within-subject factor and group as between-subject factor. When a significant time*treatment interaction was observed, post-hoc analysis with Bonferroni correction was applied to identify significant within-group effects. All variables were checked for normal distribution and were Ln-transformed to satisfy conditions of normality. All data are presented as means \pm SEM. Calculations were done using SPSS 21 for Mac OS X (IBM, Chicago, IL, USA). $\mathrm{P}<0.05$ was considered statistically significant.

\section{RESULTS}

\section{Anthropometric and clinical characteristics}

Subject characteristics before and after the 12-weeks supervised, progressive exercise training program are summarized in Table 1. At baseline, fasting plasma glucose $(P=0.009)$ and HOMA-IR $(P=0.016)$ were significantly higher, whereas peripheral $(P<0.001)$, adipose tissue $(P=0.048)$ and hepatic insulin sensitivity $(P<0.001)$ were significantly lower in the obese metabolically compromised as compared to age and BMI-matched obese metabolically healthy controls (Table 1). As expected, $\mathrm{VO}_{2 \max }\left(P_{\text {time }}<0.001\right), W_{\max }\left(P_{\text {time }}<0.001\right)$ and $1 \mathrm{RM}\left(P_{\text {time }}<0.001\right)$ were significantly increased following the exercise training intervention in both groups. The training program significantly improved peripheral insulin sensitivity to a similar extent in all subjects $\left(P_{\text {time }}=0.015 ; P_{\text {time }}{ }^{*}\right.$ group $\left.=0.106\right)$, but did not significantly affect hepatic $\left(P_{\text {time }}=0.213\right)$ and adipose tissue insulin sensitivity $\left(P_{\text {time }}=0.943\right)$ (Table 1). Furthermore, total fat mass $\left(P_{\text {time }}=0.037\right)$ and body fat percentage $\left(P_{\text {time }}=0.008\right)$ were slightly but significantly decreased, whereas body weight $\left(P_{\text {time }}=0.866\right)$, BMI $\left(P_{\text {time }}=0.890\right)$, fat free mass $\left(P_{\text {time }}=0.309\right)$ and plasma glucose, NEFA and TAG concentrations remained unaltered after the training intervention (Table 1). 


\section{Adipocyte morphology}

At baseline, no differences in mean adipocyte size and adipocyte size distribution were observed between groups (Figure $1 \mathrm{~A}$ ). The training intervention did not affect mean adipocyte size, neither in the total group $(62.9 \pm 1.4$ vs. $63.3 \pm 1.4 \mu \mathrm{m}$, $P=0.860$, Figure $1 A$ ) nor in both groups separately (Figure $1 A$ ). In line, adipocyte size distribution was not affected by the exercise training in the total group (Figure 1B) and subgroups (Figure 1C).

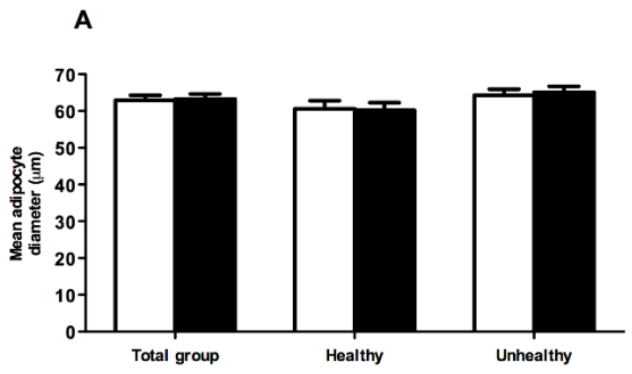

B

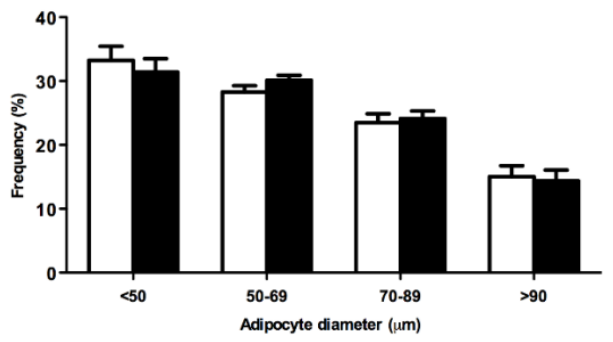

C

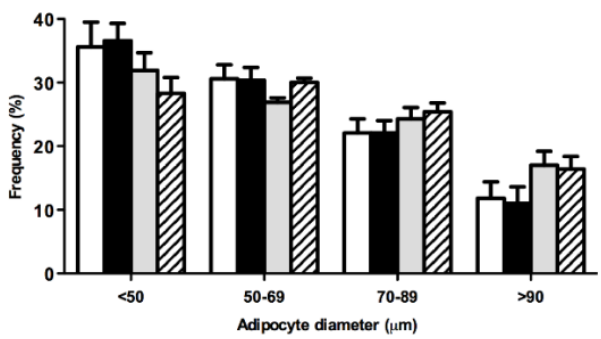

Figure 1. Exercise training-induced effects on adipose tissue morphology.

Mean adipocyte size $(\mathbf{A})$ and adipocyte size distribution in the total group (B); adipocyte size distribution in the metabolically healthy and metabolically compromised subjects $(\mathbf{C})$.

Panel A and B: white bars, baseline values; black bars, post-intervention values.

Panel C: white and grey bars, baseline values of the metabolically healthy and compromised subjects, respectively; black and striped bars, post-intervention values of the metabolically healthy and compromised subjects, respectively. 


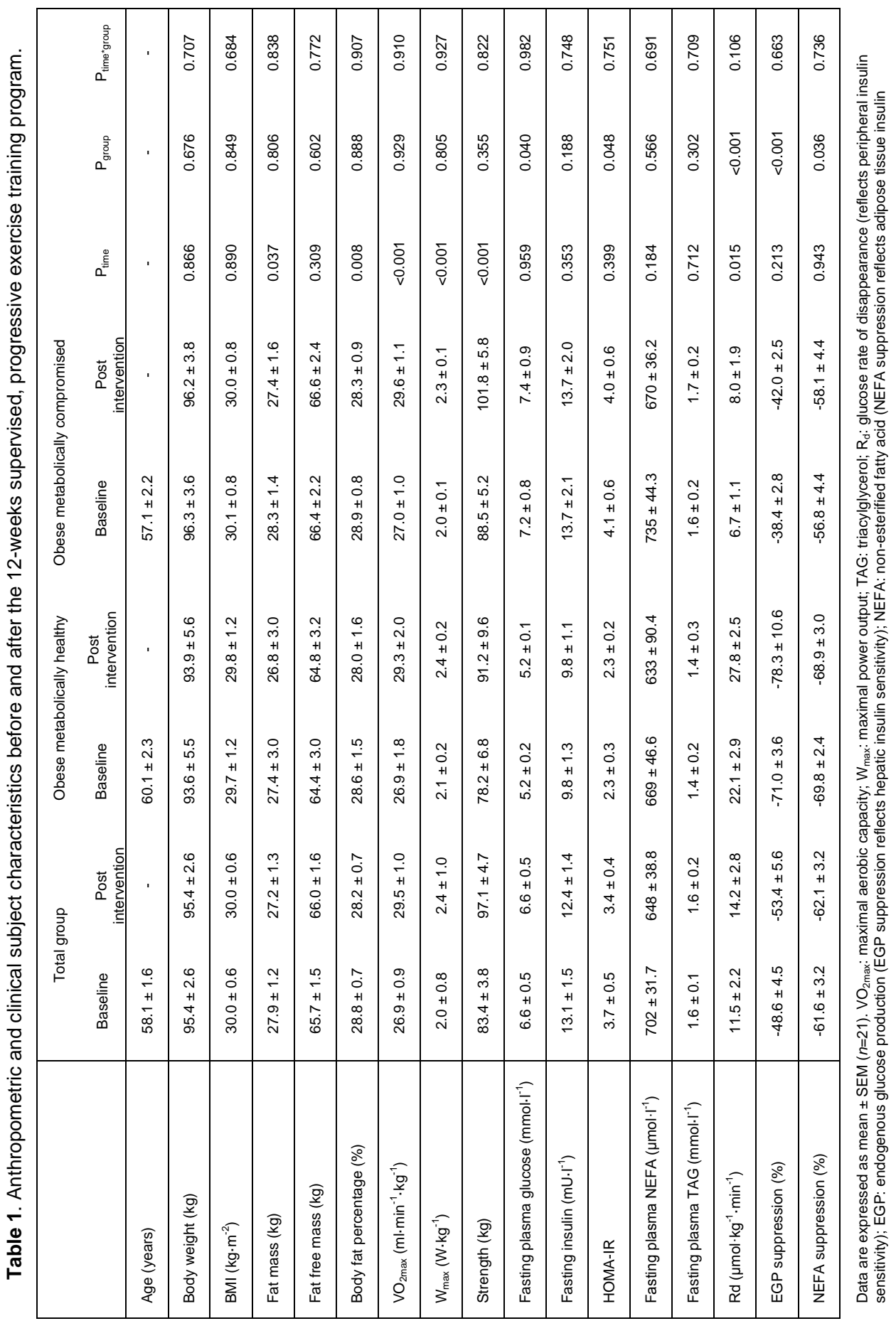




\section{Gene expression}

At baseline, gene expression of $H S L(0.35 \pm 0.08$ vs. $0.45 \pm 0.11, P=0.005)$, CGI$58(0.39 \pm 0.04$ vs. $0.75 \pm 0.19, \mathrm{P}<0.001)$ and $P G C-1 \alpha(-0.35 \pm 0.22$ vs. $0.00 \pm$ $0.13, P=0.037$ ) was significantly lower in the obese metabolically compromised as compared to obese metabolically healthy individuals. In the total group, the exercise training did not alter adipose tissue gene expression of $A T G L$ $\left(\mathrm{P}_{\text {time }}=0.332\right.$, Figure $\left.2 \mathrm{~A}\right), H S L\left(\mathrm{P}_{\text {time }}=0.862\right.$, Figure $\left.2 \mathrm{~B}\right), P L I N 1\left(\mathrm{P}_{\text {time }}=0.614\right.$, Figure $2 \mathrm{C})$ and CGI-58 $\left(\mathrm{P}_{\text {time }}=0.546\right.$, Figure $\left.2 \mathrm{D}\right)$. Furthermore, the inflammatory markers $T N F \alpha\left(\mathrm{P}_{\text {time }}=0.604\right.$, Figure 2E), IL-6 $\left(\mathrm{P}_{\text {time }}=0.507\right.$, Figure $\left.2 \mathrm{~F}\right), M C P-1\left(\mathrm{P}_{\text {time }}=0.222\right.$, Figure $2 \mathrm{G}$ ) and $C D 68$ ( $\mathrm{P}_{\text {time }}=0.688$, Figure $2 \mathrm{H}$ ) were unchanged after the intervention. Next, gene expression of the browning markers CIDEA $\left(\mathrm{P}_{\mathrm{time}}=0.943\right.$, Figure 2l) and PRDM16 ( $\mathrm{P}_{\text {time }}=0.839$, Figure 2J) and $P G C-1 \alpha\left(\mathrm{P}_{\text {time }}=0.835\right.$, Figure $2 \mathrm{~K})$, a major regulator of mitochondrial biogenesis and function, remained unchanged following the exercise training. Finally, gene expression of $L E P$ $\left(\mathrm{P}_{\text {time }}=0.840\right.$, Figure $\left.2 \mathrm{~L}\right)$ and $A D I P O Q\left(\mathrm{P}_{\text {time }}=0.413\right.$, Figure $\left.2 \mathrm{M}\right)$ was also not significantly altered after the 12-weeks training program. In line, no significant differences in exercise-induced alterations in these parameters were observed between groups, except for a slight but significant change in CGI-58 $\left(P_{\text {time }}{ }^{*}\right.$ group $\left.=0.037\right)$.
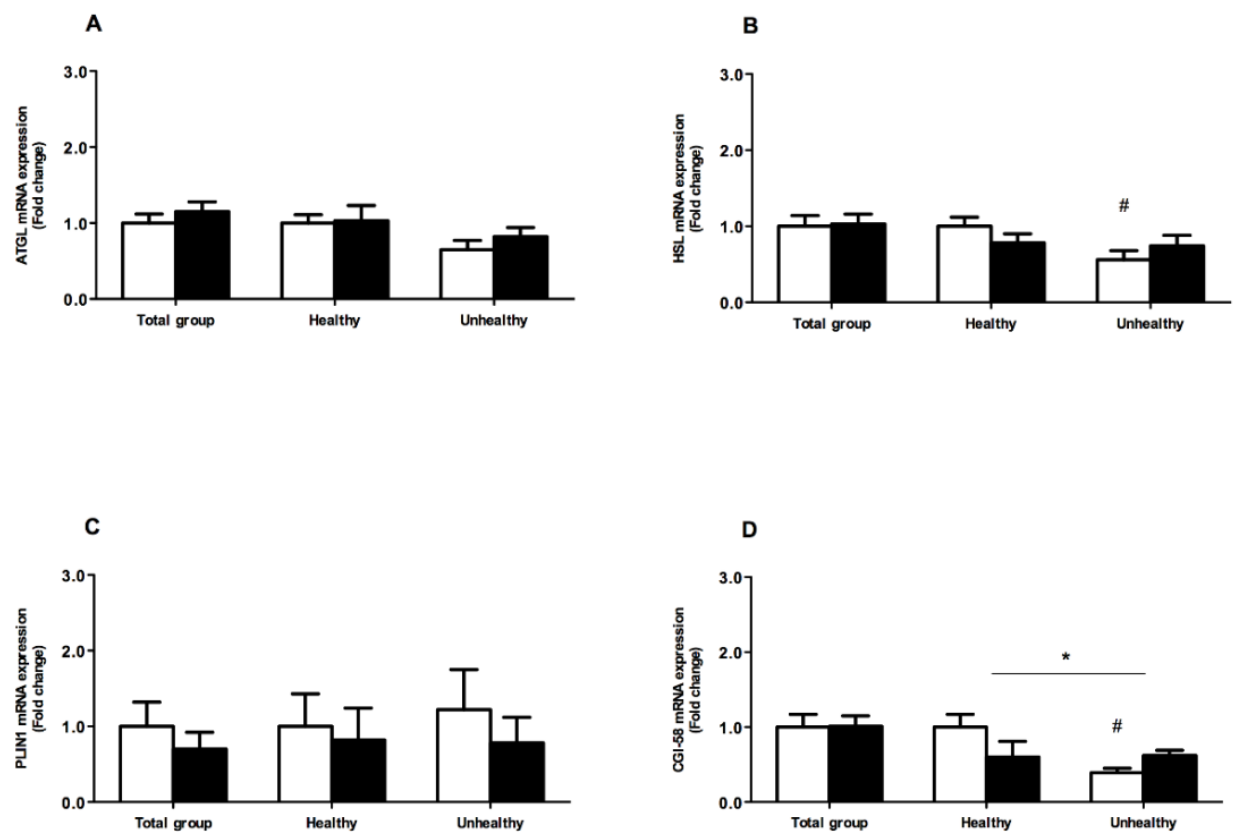

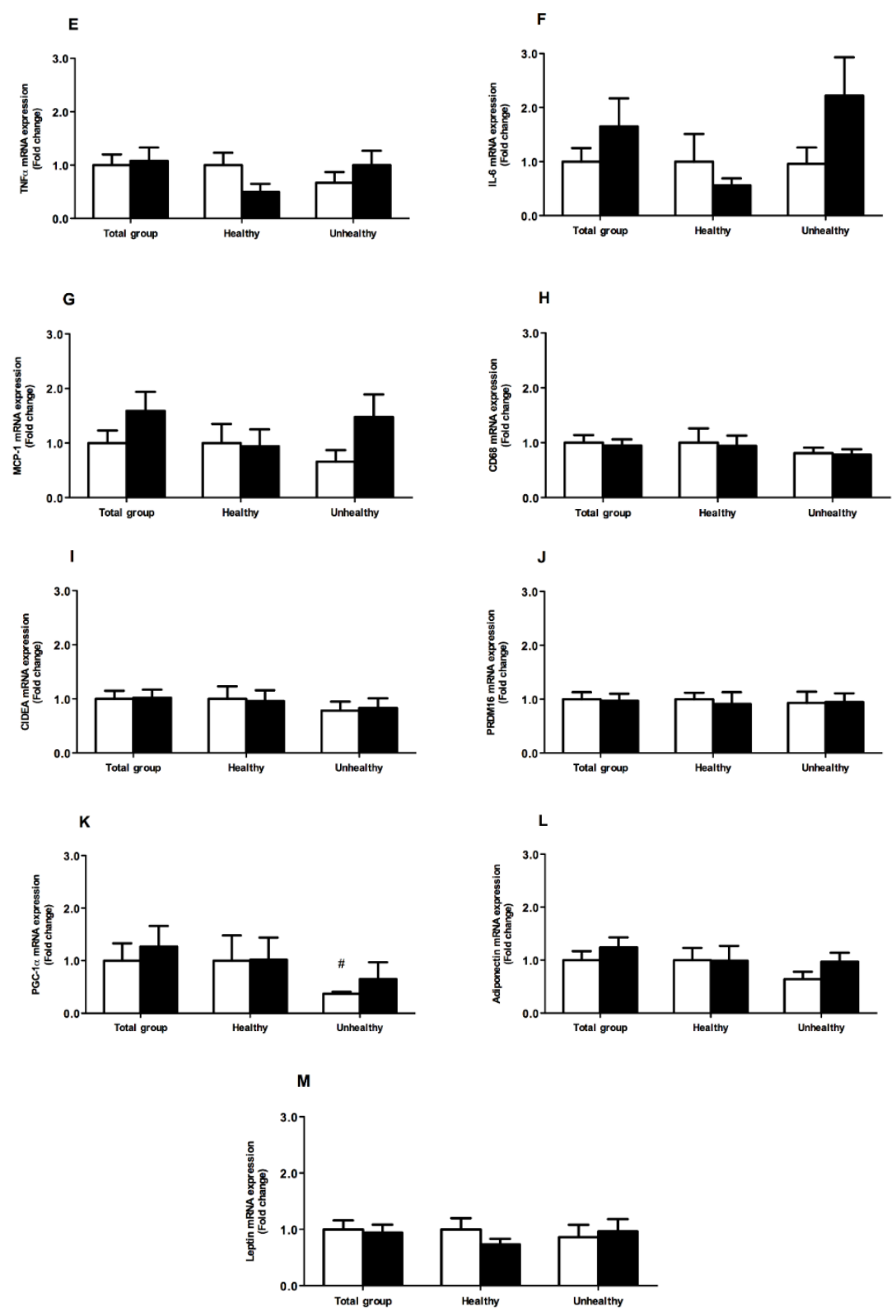

Figure 2. Exercise training-induced effects on adipose tissue gene expression.

Genes involved in lipolysis (A-D), inflammation (E-H), browning (I-J), mitochondrial biogenesis and function (K) and adipokine expression (L-M) are expressed as fold change relative to the baseline values of the total group and of the obese metabolically healthy control group.

${ }^{*} \mathrm{P}_{\text {time }}{ }_{\text {tgroup }}<0.05$; \#P<0.05 compared to baseline value of obese metabolically healthy control group. White bars, baseline values; black bars, post-intervention values. 


\section{Protein expression}

At baseline, total OXPHOS protein content was not significantly different between groups $(\mathrm{P}=0.176)$. In the total group, total OXPHOS protein expression remained unchanged following the training program $(27.0 \pm 9.7$ vs. $24.9 \pm 6.9 \mathrm{AU}$, $P_{\text {time }}=0.826$, Figure $\left.3 A\right)$. More specific, OXPHOS complex I ( $2.9 \pm 1.2$ vs. $2.9 \pm 1.0$ $\mathrm{AU}, \mathrm{P}_{\text {time }}=0.857$, Figure 3B), complex II $\left(9.2 \pm 2.4\right.$ vs. $8.9 \pm 1.7 \mathrm{AU}, \mathrm{P}_{\text {time }}=0.804$, Figure $3 \mathrm{C})$, complex III $\left(4.7 \pm 2.7\right.$ vs. $2.1 \pm 1.0 \mathrm{AU}, \mathrm{P}_{\text {time }}=0.549$, Figure $\left.3 \mathrm{D}\right)$, complex IV $\left(0.8 \pm 0.2\right.$ vs. $0.9 \pm 0.3 \mathrm{AU}, \mathrm{P}_{\text {time }}=0.870$, Figure $\left.3 \mathrm{E}\right)$ and complex $\mathrm{V}$ $\left(14.5 \pm 6.8\right.$ vs. $13.3 \pm 5.3 \mathrm{AU}, \mathrm{P}_{\text {time }}=0.666$, Figure $3 \mathrm{~F}$ ) were not affected. In line, no significant differences in exercise-induced alterations in OXPHOS protein complexes were observed between groups (Figure 3A-F).
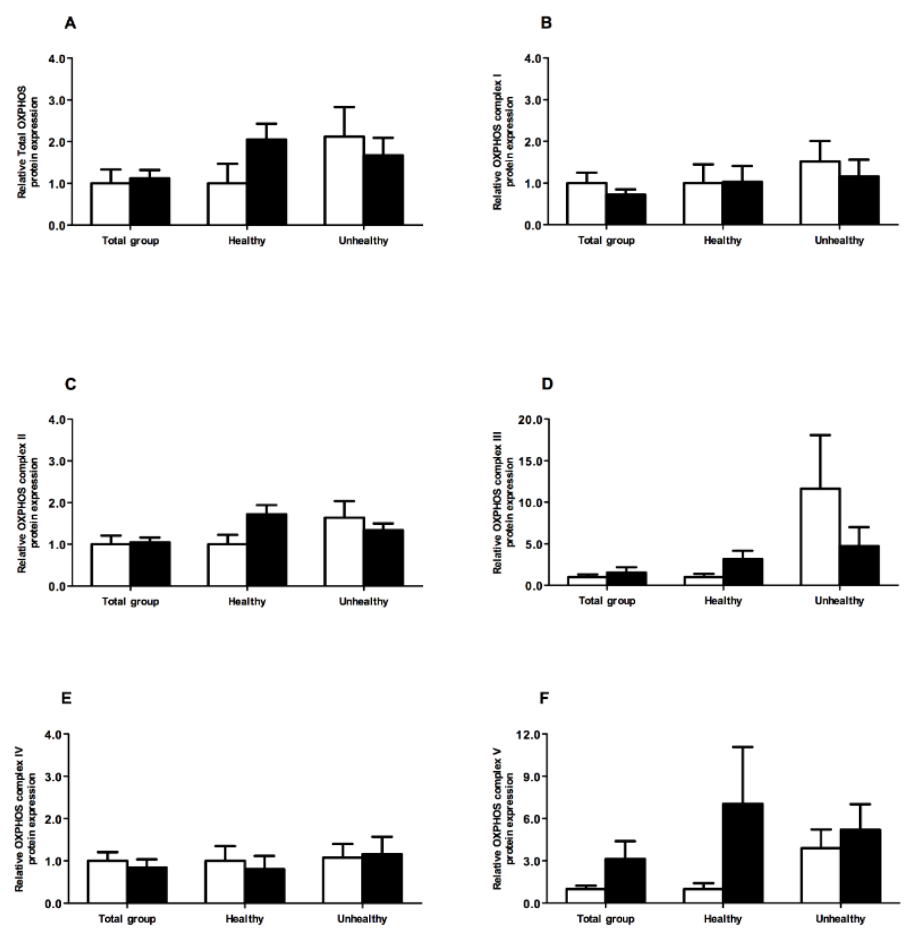

Figure 3. Exercise training-induced effects on adipose tissue mitochondrial oxidative phosphorylation (OXPHOS) protein expression.

Protein content of total OXPHOS (A), OXPHOS complex I (B), OXPHOS complex II (C), OXPHOS complex III (D), OXPHOS complex IV (E) and OXPHOS complex V (F), expressed as fold change relative to baseline values of the total group (time effect) and of the obese metabolically healthy group (time*treatment interaction).

White bars, baseline values; black bars, post-intervention values. 


\section{Ex vivo adipocyte lipolysis}

The potency of salbutamol to stimulate lipolysis was determined by its $\mathrm{EC}_{50}$, which represents the concentration of agonist inducing $50 \%$ of its maximal lipolytic response. The dose-response curve for salbutamol on ex vivo adipocyte lipolysis is presented in supplementary Figure S1. In the total group, the training intervention did not induce a significant change in basal lipolysis $(7.0 \pm 0.9$ vs. $5.9 \pm 0.6 \mu \mathrm{mol}$ glycerol $\cdot 10^{7}$ cells $^{-1} \cdot 2 \mathrm{~h}$ incubation $\left.{ }^{-1}, P=0.108\right)$, maximal lipolysis (18.1 \pm 2.1 vs. 14.5 $\pm 1.9 \mu \mathrm{mol}$ glycerol $\cdot 10^{7}$ cells $^{-1} \cdot 2 \mathrm{~h}$ incubation $\left.{ }^{-1}, P=0.111\right)$ or the potency of salbutamol $\left(-\log \mathrm{EC}_{50}\right)(6.0 \pm 0.2$ vs. $5.8 \pm 0.3, P=0.555)$.

\section{DISCUSSION}

The aim of the present study was to investigate the effects of a 12-weeks supervised, progressive exercise training program on the abdominal subcutaneous adipose tissue phenotype in metabolically healthy and metabolically compromised, well-phenotyped obese individuals. Here, we demonstrate that exercise training did neither alter abdominal subcutaneous adipocyte morphology and adipose tissue gene expression of markers for mitochondrial biogenesis/function, browning, lipolysis, inflammation and adipokines, adipose tissue OXPHOS protein content, nor $\beta_{2}$-adrenergic stimulation of adipocyte lipolysis in obese subjects, irrespective of baseline metabolic status. These data suggest that alterations in the phenotype of abdominal subcutaneous adipose tissue do not significantly contribute to the exercise-induced improvement in peripheral insulin sensitivity in obese men when adipose tissue mass is only slightly reduced $(\sim 0.7 \mathrm{~kg})$.

The training program induced a significant increase in aerobic capacity, maximal power output and maximal muscle strength, indicating that the supervised, progressive nature of the program was successful regarding enhancement of physical fitness. This was accompanied by a slight but significant decrease in fat mass and body fat percentage. In agreement with the findings in a larger study population [31], we observed that peripheral insulin sensitivity was significantly increased, whereas hepatic and adipose tissue insulin sensitivity remained unchanged after the training program.

Exercise training interventions may affect adipocyte morphology in humans [30]. We demonstrated that a 12-weeks exercise training intervention did not significantly alter mean adipocyte size or adipocyte size distribution, despite a 0.7 $\mathrm{kg}$ decrease in total fat mass. In contrast, Despres et al. [32] demonstrated that 20 weeks of endurance training decreased mean adipocyte size in young men, but not in women. Importantly, they observed a more pronounced reduction in body weight $(\sim 3.0 \mathrm{~kg})$. Thus, a more prolonged intervention period, leading to a more pronounced decrease in adipose tissue mass, seems necessary to induce beneficial changes in adipocyte morphology.

Since an altered rate of lipolysis is one of the characteristics of adipose tissue dysfunction and relates to peripheral insulin resistance [8], we determined adipose tissue gene expression of lipolytic enzymes and genes encoding lipid dropletassociated proteins. At baseline, gene expression of HSL and CGI-58 was lower in obese metabolically compromised as compared to obese metabolically healthy subjects. These findings are in line with previous studies from our group and 
others, showing a reduced expression of lipolytic genes in obese insulin resistant [33] and patients with T2DM [34] as compared to obese insulin sensitive individuals. Conflicting results on the impact of exercise training on basal and stimulated adipose tissue lipolysis in humans has been reported, as extensively reviewed [30]. Here, we show that exercise training did not alter the expression of genes related to lipolysis under fasting conditions in obese individuals, irrespective of baseline metabolic status, except for a slight change in CGI-58. It has previously been demonstrated that $\beta_{2}$-adrenergic stimulation of lipolysis is impaired in obese as compared to lean subjects, whereas $\beta_{1}$-adrenergic receptor sensitivity was comparable between groups [35]. We therefore determined if exercise training altered $\beta_{2}$-adrenergic sensitivity of lipolysis. In agreement with unchanged adipose tissue gene expression of lipolytic markers, the potency of the $\beta_{2}$-adrenergic receptor agonist salbutamol to stimulate abdominal subcutaneous adipocyte lipolysis was comparable before and after the exercise training program. Furthermore, basal and maximal $\beta_{2}$-adrenergic receptor-mediated lipolysis remained unchanged after exercise training. The present findings are in agreement with a previous study showing no improvement of $\beta_{2}$-adrenergic stimulation of lipolysis after 12 weeks of training in obese non-diabetic men [36]. However, in contrast to the present findings, a decreased basal lipolysis was found after exercise training in the latter study [36]. This may be explained by the modest loss of fat mass in the present study, which did not result in a reduction in adipocyte size. Indeed, it has previously been demonstrated that substantial weight loss, which decreased adipocyte size, increased and normalized the sensitivity to catecholamine-stimulated lipolysis in obese subjects [37].

In addition to impairments in lipolysis, a pro-inflammatory phenotype of adipose tissue is associated with insulin resistance in obese subjects and T2DM patients [68]. In the present study, no changes in macrophage infiltration and inflammatory markers in adipose tissue were found following exercise training. The absence of alterations in the inflammatory profile after exercise training is in agreement with most previous studies in obese subjects [22, 23, 25, 26]. Furthermore, we found no effects of exercise training on adipose tissue gene expression of leptin and adiponectin, which is in accordance with observations in both lean and overweight/obese humans [25, 26, 38]. Nevertheless, some studies have shown a reduction in adipose tissue gene expression of MCP-1 [22] and an increase in adiponectin expression [23] following exercise training in obese subjects. The present findings further support the notion that a reduction in adipocyte size is required to achieve beneficial changes in the adipose tissue phenotype.

Rodent studies demonstrated that increasing brown adipose tissue mass/activity or inducing browning of white adipose tissue via cold exposure or other stimuli might be a promising strategy for the treatment of obesity and obesity-related impairments in glucose homeostasis [39]. Interestingly, physical exercise has been shown to induce browning of white adipose tissue in rodents, possibly via the secretion of the myokine irisin [15], although findings are controversial [40], or via increased natriuretic peptide concentrations [41]. However, the expression of browning markers in white adipose tissue of endurance-trained athletes was not different from lean sedentary controls [42]. In the present study, the expression of PRDM16 and CIDEA, markers of beiging/browning of white adipose tissue, did not change following the intervention. In agreement with our findings, no exercise 
training-induced changes in browning markers in white adipose tissue were found in lean $[43,44]$ and overweight $[20,44]$ subjects.

The oxidative phenotype of white adipose tissue is impaired in obesity and seems related to an altered glucose homeostasis in rodents and humans [45-48]. Here, we found that baseline gene expression of $P G C-1 \alpha$ was significantly lower in obese metabolically compromised as compared to metabolically healthy obese subjects, although this did not translate into significant differences in OXPHOS protein content between these BMI-matched groups. Furthermore, the exercise training did not induce alterations in adipose tissue gene expression of $P G C-1 \alpha$, which is in accordance with most [20,38, 43], but not all previous studies [19, 49]. Finally, we observed no significant exercise-induced alterations in adipose tissue OXPHOS protein content after the training intervention, which is in line with highintensity interval training in healthy lean [43] and overweight [50] subjects. In contrast to human data, previous rodent studies showed that endurance training had beneficial effects on intra-abdominal and epididymal white adipose tissue mitochondrial activity in rats [12], indicating species differences in mitochondrial protein expression and/or function in response to exercise.

In conclusion, the present study demonstrated that a 12-weeks supervised, progressive exercise training intervention, which improved physical fitness and peripheral insulin sensitivity, had no significant effects on abdominal subcutaneous adipocyte morphology, adipose tissue gene and protein expression of markers related to adipose tissue function, nor $\beta_{2}$-adrenergic sensitivity in obese subjects, irrespective of their baseline metabolic status. Noteworthy, we cannot exclude that exercise training may induce beneficial alterations in the adipose tissue phenotype after a more prolonged intervention period, leading to a more pronounced loss of fat mass, or in other fat depots. 


\section{REFERENCES}

1. Kopelman PG. Obesity as a medical problem. Nature. 2000;404(6778):635-43.

2. Mann S, Beedie C, Balducci S, Zanuso S, Allgrove J, Bertiato F, Jimenez A. Changes in insulin sensitivity in response to different modalities of exercise: a review of the evidence. Diabetes Metab Res Rev. 2014;30(4):257-68.

3. Roberts CK, Little JP, Thyfault JP. Modification of insulin sensitivity and glycemic control by activity and exercise. Med Sci Sports Exerc. 2013;45(10):1868-77.

4. Meex RC, Schrauwen-Hinderling VB, Moonen-Kornips E, Schaart G, Mensink M, Phielix E, van de Weijer T, Sels JP, Schrauwen P, Hesselink MK. Restoration of muscle mitochondrial function and metabolic flexibility in type 2 diabetes by exercise training is paralleled by increased myocellular fat storage and improved insulin sensitivity. Diabetes. 2010;59(3):572-9.

5. Colberg SR, Sigal RJ, Fernhall B, Regensteiner JG, Blissmer BJ, Rubin RR, ChasanTaber L, Albright AL, Braun B, American College of Sports M, American Diabetes A. Exercise and type 2 diabetes: the American College of Sports Medicine and the American Diabetes Association: joint position statement. Diabetes Care. 2010;33(12):e147-67.

6. Goossens $\mathrm{GH}$. The role of adipose tissue dysfunction in the pathogenesis of obesityrelated insulin resistance. Physiol Behav. 2008;94(2):206-18.

7. Rosen ED, Spiegelman BM. What we talk about when we talk about fat. Cell. 2014;156(1-2):20-44.

8. Stinkens R, Goossens GH, Jocken JW, Blaak EE. Targeting fatty acid metabolism to improve glucose metabolism. Obes Rev. 2015;16(9):715-57.

9. Stanford KI, Middelbeek RJ, Goodyear LJ. Exercise Effects on White Adipose Tissue: Beiging and Metabolic Adaptations. Diabetes. 2015;64(7):2361-8.

10. Sutherland LN, Bomhof MR, Capozzi LC, Basaraba SA, Wright DC. Exercise and adrenaline increase PGC-1\{alpha\} mRNA expression in rat adipose tissue. J Physiol. 2009;587(Pt 7):1607-17.

11. Trevellin E, Scorzeto M, Olivieri M, Granzotto M, Valerio A, Tedesco L, Fabris R, Serra R, Quarta M, Reggiani C, Nisoli E, Vettor R. Exercise training induces mitochondrial biogenesis and glucose uptake in subcutaneous adipose tissue through eNOS-dependent mechanisms. Diabetes. 2014;63(8):2800-11.

12. Stallknecht B, Vinten J, Ploug T, Galbo H. Increased activities of mitochondrial enzymes in white adipose tissue in trained rats. Am J Physiol. 1991;261(3 Pt 1):E410-4.

13. Stanford KI, Middelbeek RJ, Townsend KL, Lee MY, Takahashi H, So K, Hitchcox KM, Markan KR, Hellbach K, Hirshman MF, Tseng YH, Goodyear LJ. A novel role for subcutaneous adipose tissue in exercise-induced improvements in glucose homeostasis. Diabetes. 2015;64(6):2002-14.

14. Vernochet C, Mourier A, Bezy O, Macotela Y, Boucher J, Rardin MJ, An D, Lee KY, Ilkayeva OR, Zingaretti CM, Emanuelli B, Smyth G, Cinti S, Newgard CB, Gibson BW, Larsson NG, Kahn CR. Adipose-specific deletion of TFAM increases mitochondrial oxidation and protects mice against obesity and insulin resistance. Cell Metab. 2012;16(6):765-76.

15. Bostrom P, Wu J, Jedrychowski MP, Korde A, Ye L, Lo JC, Rasbach KA, Bostrom EA, Choi JH, Long JZ, Kajimura S, Zingaretti MC, Vind BF, Tu H, Cinti S, Hojlund K, Gygi SP, Spiegelman BM. A PGC1-alpha-dependent myokine that drives brown-fatlike development of white fat and thermogenesis. Nature. 2012;481(7382):463-8.

16. Cao L, Choi EY, Liu X, Martin A, Wang C, Xu X, During MJ. White to brown fat phenotypic switch induced by genetic and environmental activation of a hypothalamic-adipocyte axis. Cell Metab. 2011;14(3):324-38. 
17. Zachwieja JJ, Hendry SL, Smith SR, Harris RB. Voluntary wheel running decreases adipose tissue mass and expression of leptin mRNA in Osborne-Mendel rats. Diabetes. 1997;46(7):1159-66.

18. Bradley RL, Jeon JY, Liu FF, Maratos-Flier E. Voluntary exercise improves insulin sensitivity and adipose tissue inflammation in diet-induced obese mice. Am J Physiol Endocrinol Metab. 2008;295(3):E586-94.

19. Ruschke K, Fishbein L, Dietrich A, Kloting N, Tonjes A, Oberbach A, Fasshauer M, Jenkner J, Schon MR, Stumvoll M, Bluher M, Mantzoros CS. Gene expression of PPARgamma and PGC-1alpha in human omental and subcutaneous adipose tissues is related to insulin resistance markers and mediates beneficial effects of physical training. Eur J Endocrinol. 2010;162(3):515-23.

20. Ronn T, Volkov P, Tornberg A, Elgzyri T, Hansson O, Eriksson KF, Groop L, Ling C. Extensive changes in the transcriptional profile of human adipose tissue including genes involved in oxidative phosphorylation after a 6-month exercise intervention. Acta Physiol (Oxf). 2014;211(1):188-200.

21. Bluher M, Williams CJ, Kloting N, Hsi A, Ruschke K, Oberbach A, Fasshauer M, Berndt J, Schon MR, Wolk A, Stumvoll M, Mantzoros CS. Gene expression of adiponectin receptors in human visceral and subcutaneous adipose tissue is related to insulin resistance and metabolic parameters and is altered in response to physical training. Diabetes Care. 2007;30(12):3110-5.

22. Sjogren P, Sierra-Johnson J, Kallings LV, Cederholm T, Kolak M, Halldin M, Brismar $\mathrm{K}$, de Faire U, Hellenius ML, Fisher RM. Functional changes in adipose tissue in a randomised controlled trial of physical activity. Lipids Health Dis. 2012;11:80.

23. Trachta P, Drapalova J, Kavalkova P, Touskova V, Cinkajzlova A, Lacinova Z, Matoulek M, Zelinka T, Widimsky J, Jr., Mraz M, Haluzik M. Three months of regular aerobic exercise in patients with obesity improve systemic subclinical inflammation without major influence on blood pressure and endocrine production of subcutaneous fat. Physiol Res. 2014;63 Suppl 2:S299-308.

24. Moghadasi M, Mohebbi H, Rahmani-Nia F, Hassan-Nia S, Noroozi H, Pirooznia N. High-intensity endurance training improves adiponectin mRNA and plasma concentrations. Eur J Appl Physiol. 2012;112(4):1207-14.

25. Klimcakova E, Polak J, Moro C, Hejnova J, Majercik M, Viguerie N, Berlan M, Langin $\mathrm{D}$, Stich V. Dynamic strength training improves insulin sensitivity without altering plasma levels and gene expression of adipokines in subcutaneous adipose tissue in obese men. J Clin Endocrinol Metab. 2006;91(12):5107-12.

26. Polak J, Klimcakova E, Moro C, Viguerie N, Berlan M, Hejnova J, Richterova B, Kraus I, Langin D, Stich V. Effect of aerobic training on plasma levels and subcutaneous abdominal adipose tissue gene expression of adiponectin, leptin, interleukin 6, and tumor necrosis factor alpha in obese women. Metabolism. 2006;55(10):1375-81.

27. Hulver MW, Zheng D, Tanner CJ, Houmard JA, Kraus WE, Slentz CA, Sinha MK, Pories WJ, MacDonald KG, Dohm GL. Adiponectin is not altered with exercise training despite enhanced insulin action. Am $J$ Physiol Endocrinol Metab. 2002;283(4):E861-5.

28. Richterova B, Stich V, Moro C, Polak J, Klimcakova E, Majercik M, Harant I, Viguerie $N$, Crampes $F$, Langin D, Lafontan M, Berlan M. Effect of endurance training on adrenergic control of lipolysis in adipose tissue of obese women. J Clin Endocrinol Metab. 2004;89(3):1325-31.

29. Covington JD, Bajpeyi S, Moro C, Tchoukalova YD, Ebenezer PJ, Burk DH, Ravussin E, Redman LM. Potential effects of aerobic exercise on the expression of perilipin 3 in the adipose tissue of women with polycystic ovary syndrome: a pilot study. Eur $\mathrm{J}$ Endocrinol. 2015;172(1):47-58. 
30. Thompson D, Karpe F, Lafontan M, Frayn K. Physical activity and exercise in the regulation of human adipose tissue physiology. Physiol Rev. 2012;92(1):157-91.

31. Brouwers B, Schrauwen-Hinderling VB, Jelenik T, Gemmink A, Havekes B, Bruls YM, Dahlmans D, Roden M, Hesselink MK, Schrauwen P. Metabolic disturbances of nonalcoholic fatty liver resemble the alterations typical for overt type 2 diabetes. Clin Sci (Lond). 2017;131(15):1905-17.

32. Despres JP, Bouchard C, Savard R, Tremblay A, Marcotte M, Theriault G. The effect of a 20 -week endurance training program on adipose-tissue morphology and lipolysis in men and women. Metabolism. 1984;33(3):235-9.

33. Jocken JW, Langin D, Smit E, Saris WH, Valle C, Hul GB, Holm C, Arner P, Blaak $\mathrm{EE}$. Adipose triglyceride lipase and hormone-sensitive lipase protein expression is decreased in the obese insulin-resistant state. $J$ Clin Endocrinol Metab. 2007;92(6):2292-9.

34. Watt MJ, Carey AL, Wolsk-Petersen E, Kraemer FB, Pedersen BK, Febbraio MA. Hormone-sensitive lipase is reduced in the adipose tissue of patients with type 2 diabetes mellitus: influence of IL-6 infusion. Diabetologia. 2005;48(1):105-12.

35. Schiffelers SL, Saris WH, Boomsma F, van Baak MA. beta(1)- and beta(2)Adrenoceptor-mediated thermogenesis and lipid utilization in obese and lean men. $J$ Clin Endocrinol Metab. 2001;86(5):2191-9.

36. De Glisezinski I, Crampes F, Harant I, Berlan M, Hejnova J, Langin D, Riviere D, Stich $\mathrm{V}$. Endurance training changes in lipolytic responsiveness of obese adipose tissue. Am J Physiol. 1998;275(6 Pt 1):E951-6.

37. Reynisdottir S, Langin D, Carlstrom K, Holm C, Rossner S, Arner P. Effects of weight reduction on the regulation of lipolysis in adipocytes of women with upper-body obesity. Clin Sci (Lond). 1995;89(4):421-9.

38. Alvehus M, Boman N, Soderlund K, Svensson MB, Buren J. Metabolic adaptations in skeletal muscle, adipose tissue, and whole-body oxidative capacity in response to resistance training. Eur J Appl Physiol. 2014;114(7):1463-71.

39. Harms $M$, Seale $P$. Brown and beige fat: development, function and therapeutic potential. Nat Med. 2013;19(10):1252-63.

40. Elsen M, Raschke S, Eckel J. Browning of white fat: does irisin play a role in humans? J Endocrinol. 2014;222(1):R25-38.

41. Bordicchia M, Liu D, Amri EZ, Ailhaud G, Dessi-Fulgheri P, Zhang C, Takahashi N, Sarzani R, Collins S. Cardiac natriuretic peptides act via p38 MAPK to induce the brown fat thermogenic program in mouse and human adipocytes. J Clin Invest. 2012;122(3):1022-36.

42. Vosselman MJ, Hoeks J, Brans B, Pallubinsky H, Nascimento EB, van der Lans AA, Broeders EP, Mottaghy FM, Schrauwen P, van Marken Lichtenbelt WD. Low brown adipose tissue activity in endurance-trained compared with lean sedentary men. Int $J$ Obes (Lond). 2015;39(12):1696-702.

43. Camera DM, Anderson MJ, Hawley JA, Carey AL. Short-term endurance training does not alter the oxidative capacity of human subcutaneous adipose tissue. Eur $\mathrm{J}$ Appl Physiol. 2010;109(2):307-16.

44. Norheim F, Langleite TM, Hjorth M, Holen T, Kielland A, Stadheim HK, Gulseth HL, Birkeland KI, Jensen J, Drevon CA. The effects of acute and chronic exercise on PGC-1alpha, irisin and browning of subcutaneous adipose tissue in humans. FEBS J. 2014;281(3):739-49.

45. Heinonen S, Buzkova J, Muniandy $M$, Kaksonen $R$, Ollikainen $M$, Ismail $K$, Hakkarainen A, Lundbom J, Lundbom N, Vuolteenaho K, Moilanen E, Kaprio J, Rissanen A, Suomalainen A, Pietilainen KH. Impaired Mitochondrial Biogenesis in Adipose Tissue in Acquired Obesity. Diabetes. 2015;64(9):3135-45.

46. Semple RK, Crowley VC, Sewter CP, Laudes M, Christodoulides C, Considine RV, Vidal-Puig A, O'Rahilly S. Expression of the thermogenic nuclear hormone receptor 
coactivator PGC-1alpha is reduced in the adipose tissue of morbidly obese subjects. Int J Obes Relat Metab Disord. 2004;28(1):176-9.

47. Kusminski CM, Scherer PE. Mitochondrial dysfunction in white adipose tissue. Trends Endocrinol Metab. 2012;23(9):435-43.

48. Yin X, Lanza IR, Swain JM, Sarr MG, Nair KS, Jensen MD. Adipocyte mitochondrial function is reduced in human obesity independent of fat cell size. $J$ Clin Endocrinol Metab. 2014;99(2):E209-16.

49. Khadir A, Tiss A, Abubaker J, Abu-Farha M, Al-Khairi I, Cherian P, John J, Kavalakatt S, Warsame S, Al-Madhoun A, Al-Ghimlas F, Elkum N, Behbehani K, Dermime S, Dehbi M. MAP kinase phosphatase DUSP1 is overexpressed in obese humans and modulated by physical exercise. Am J Physiol Endocrinol Metab. 2015;308(1):E71 83.

50. Larsen S, Danielsen JH, Sondergard SD, Sogaard D, Vigelsoe A, Dybboe R, Skaaby $S$, Dela F, Helge JW. The effect of high-intensity training on mitochondrial fat oxidation in skeletal muscle and subcutaneous adipose tissue. Scand J Med Sci Sports. 2015;25(1):e59-69. 


\section{SUPPLEMENTARY MATERIAL}

\section{Protein expression analysis}

Firstly, subcutaneous adipose tissue $(500 \mathrm{mg}$ ) was ground to a fine powder under liquid nitrogen and homogenized in radioimmunoprecipitation assay buffer $(10 \mathrm{mM}$ Tris (Calbiochem)- $\mathrm{HCl}$ (Merck, Darmstadt, Germany) buffered saline (Merck) with $0,1 \%$ sodium dodecyl sulfate (SDS) (Bio-Rad Laboratories Inc, Hercules, CA, USA), $1 \%$ sodiumdeoxycholate (Sigma-Aldrich, St. Louis, MO, USA), 1\% NP-40 (Fluka) and a protease/phosphatase inhibitor cocktail (Cell Signaling Technology, Beverly, MA, USA). The homogenate was lysed on iced and vortexed for $5 \mathrm{~min}$ and centrifuged at $20,000 \mathrm{~g}$ for $30 \mathrm{~min}$ at $10^{\circ} \mathrm{C}$. The supernatant was carefully collected and aliquots were stored at $-80^{\circ} \mathrm{C}$. The protein concentration was determined by the Bradford-based protein assay (Santa Cruz Biotechnology, Dallas, TX, USA).

Next, solubilized proteins were separated on a precast gel (Criterion ${ }^{\mathrm{TM}} \mathrm{TGX}$ any kD, Bio-Rad Laboratories Inc, Hercules, CA, USA) and transferred onto a nitrocellulose membrane (Trans Blot ${ }^{\circledR}$ Turbo $^{\mathrm{TM}}$ transfer system; Bio-Rad). Differences in loading were adjusted to total protein content (via Ponceau $S$ (Sigma-Aldrich, St. Louis, MO, USA) staining) and appropriate positive controls were included.

Thereafter, quantitative western blot analysis was performed to determine the levels of the OXPHOS proteins. OXPHOS blots were probed with Total OXPHOS Antibody Cocktail (Mitoscience/Abcam, Cambridge, MA, USA) and a secondary horseradish peroxidase (HRP)-conjugated Rabbit-anti-Mouse antibody (DakoCytomation, Glostrup, Denmark). Antigen-antibody complexes were visualized using chemiluminescence $(E C L)$ by a ChemiDoc ${ }^{\mathrm{TM}} \mathrm{XRS}$ apparatus (BioRad) and analyzed with Quantity One® software (Bio-Rad), which calculated the optical density units that are expressed as average intensity ([average intensity = total intensity of the rows of pixels inside the band boundary divided by the number of rows, minus the background intensity]).

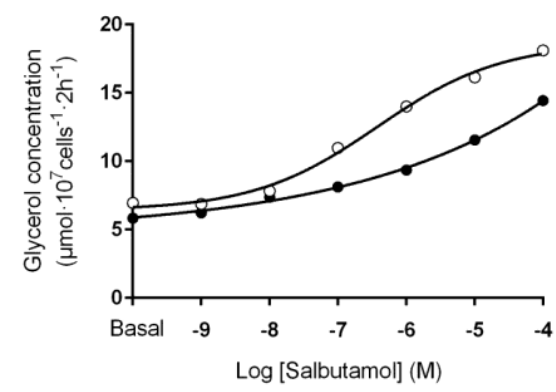

Supplementary Figure S1. The effects of salbutamol on ex vivo adipocyte lipolysis before and after exercise training in the total group $(n=15)$.

Glycerol release into the medium $\left(\mu \mathrm{mol}\right.$ glycerol $\cdot 10^{7}$ cells $^{-1} \cdot 2 \mathrm{~h}$ incubation ${ }^{-1}$ ) was used as an indicator of lipolysis.

Open circles represent baseline values; closed circles represent post-intervention values. 
Supplementary Table S1: RT-qPCR Primer Sequences

The following RT-qPCR primer sequences were used for gene expression analysis.

Upper sequences represent forward primers $\left(5^{\prime}-3^{\prime}\right)$, while lower sequences represent reverse primer $\left(5^{\prime}-3^{\prime}\right)$.

\begin{tabular}{|c|c|}
\hline Genes & Sequences \\
\hline ATGL & $\begin{array}{l}\text { GTGTCAGACGGCGAGAATG } \\
\text { TGGAGGGAGGGAGGGATG }\end{array}$ \\
\hline HSL & $\begin{array}{c}\text { GCGGATCACACAGAACCTGGAC } \\
\text { AGCAGGCGGCTTACCCTCAC }\end{array}$ \\
\hline CGI-58 & $\begin{array}{l}\text { CAGCATCCAGTCCTTACGACCA } \\
\text { GTTCAGTCCACAGTGTCGCAGA }\end{array}$ \\
\hline PLIN1 & $\begin{array}{l}\text { CTCTCGATACACCGTGCAGA } \\
\text { TGGTCCTCATGATCCTCCTC }\end{array}$ \\
\hline TNFa & $\begin{array}{l}\text { CCGAGTGACAAGCCTGTAGC } \\
\text { GAGGACCTGGGAGTAGATGAG }\end{array}$ \\
\hline IL-6 & $\begin{array}{l}\text { AAATTCGGTACATCCTCGACGG } \\
\text { GGAAGGTTCAGGTTGTTTTCTGC }\end{array}$ \\
\hline MCP-1 & $\begin{array}{l}\text { CCCCAGTCACCTGCTGTTAT } \\
\text { TCCTGAACCCACTTCTGCTT }\end{array}$ \\
\hline CD68 & $\begin{array}{l}\text { CCCTATGGACACCTCAGCTTT } \\
\text { GAAGGACACATTGTACTCCACC }\end{array}$ \\
\hline CIDEA & $\begin{array}{l}\text { TCAGACCTTGGGAGACAACACG } \\
\text { CGAAGGTGACTCTCGCTATTCC }\end{array}$ \\
\hline PRDM16 & $\begin{array}{l}\text { CAGCCAATCTCACCAGACACCT } \\
\text { GTGGCACTTGAAAGGCTTCTCC }\end{array}$ \\
\hline PGC-1a & $\begin{array}{l}\text { TCTGAGTCTGTATGGAGTGACAT } \\
\text { CCAAGTCGTTCACATCTAGTTCA }\end{array}$ \\
\hline ADIPOQ & $\begin{array}{c}\text { TGGTGAGAAGGGTGAGAA } \\
\text { AGATCTTGGTAAAGCGAATG }\end{array}$ \\
\hline LEP & $\begin{array}{l}\text { GAACCCTGTGCGGATTCTTGT } \\
\text { TCCATCTTGGATAAGGTCAGGAT }\end{array}$ \\
\hline
\end{tabular}


CHAPTER 7 


\section{Coordinated regulation of adipose tissue adrenergic- and non-adrenergic- mediated lipolysis during exercise in lean and obese individuals: the effect of exercise training}

Stinkens R. ${ }^{*}$, Verboven K. ${ }^{*}$, Hansen D., Wens I., Frederix I., Eijnde B.O., Jocken J.W., Goossens G.H.", Blaak E.E."

* Shared first authorship, " Shared last authorship

Submitted 


\section{ABSTRACT}

Background: Adipose tissue dysfunction, which includes impairments in (adipose tissue) lipolysis, contributes to insulin resistance. Subcutaneous adipose tissue (SCAT) lipolysis in obesity is characterized by catecholamine resistance and an impaired ANP responsiveness. It remains to be established whether exercise training improves non-adrenergically-mediated lipolysis, next to the adrenergic pathway, in metabolically compromised conditions. The aim of the present study was to investigate the effect of local combined $\alpha$ - and $\beta$-adrenergic receptor blockade on SCAT lipolysis in obese insulin sensitive (IS), obese insulin resistant (IR) and age-matched lean IS men. Moreover, obese individuals underwent endurance and resistance exercise training to improve metabolic profile and (non-) adrenergically-mediated SCAT lipolysis.

Methods: Abdominal SCAT lipolysis was investigated in 10 obese IS, 10 obese IR and 10 age-matched lean IS men using microdialysis in the presence or absence of local combined $\alpha$ - and $\beta$-adrenergic receptor blockade at rest, during $60 \mathrm{~min}$ of low-intense (40\% VO2max) endurance-type exercise and recovery. Systemic responses were investigated using venous blood sampling. Obese individuals participated in a supervised, endurance and resistance exercise training intervention for 12 weeks (3 sessions/week) after which the microdialysis measurements were repeated in obese IR men.

Results: Exercise-induced increase in abdominal SCAT lipolysis (expressed as total area under the curve) was more pronounced in obese IS (81\%) and IR (34\%) as compared to lean individuals $\left(P_{\text {group }}=0.012\right)$. Abdominal SCAT lipolysis was significantly reduced $(\sim 40 \%)$ following local combined $\alpha$-/ $\beta$-adrenoceptor blockade in obese IS individuals only. Despite improvements in body composition, physical fitness and exercise-induced changes in circulating free fatty acids, lactate and adrenalin, exercise intervention did not significantly affect (non-)adrenergicallymediated lipolysis in abdominal SCAT of obese IR individuals.

Conclusion: Our findings indicate a major contribution of non-adrenergically mediated lipolysis during exercise in abdominal SCAT of lean and obese individuals. Furthermore, a 12-week exercise training program improved metabolic profile and body composition in obese individuals, but did not affect abdominal SCAT lipolysis. 


\section{INTRODUCTION}

Adipose tissue (AT) dysfunction is commonly observed in human obesity and contributes to insulin resistance (IR) and chronic metabolic diseases, including cardiovascular disease, type 2 diabetes mellitus (T2D) and certain types of cancer $[1,2]$. Disturbances in AT lipid metabolism, including a decreased lipid uptake and impairments in lipid mobilization are closely linked to ectopic fat deposition and obesity-related IR [3]. An important function of the AT is to release fatty acids through lipolysis $[4,5]$, especially during fasting and increased energy demanding conditions such as exercise. Multiple endocrine factors affect the activity of lipid droplet-associated proteins and lipases, thereby regulating the release of free fatty acids (FFA) and glycerol [6]. However, impairments in the regulation of lipolysis have been identified in subcutaneous AT (SCAT) of obese humans [7], including a blunted catecholamine-mediated lipolysis $[8,9]$. More specific, $\beta$-adrenergicallymediated lipolysis is reduced [9] and inhibitory $\alpha_{2}$-adrenoceptors become predominant on adipocytes in the obese insulin resistant state [10] [11], leading to a blunted adrenergically-mediated lipolysis [9, 12]. Of interest, local $\beta$-adrenergic blockade (alone or in combination with $\alpha_{2}$-adrenergic blockade) in SCAT, inhibits exercise-induced lipolysis only to a minor extent at low-to-moderate intensities in healthy lean [13-15] and overweight individuals [16]. In this respect, Moro et al. [16] demonstrated that non-adrenergically-mediated lipolysis in SCAT substantially contributes to lipid mobilization during exercise in healthy young lean men [15] and healthy young overweight men [16]. Other key regulators of lipolysis are insulin [17] and lactate [18], which both exert an inhibitory role in the physiological control of AT during exercise [17-19].

More recently, evidence has emerged that natriuretic peptides (NP) not only affect the cardiovascular system, but also have pronounced effects in several key metabolic organs such as AT and skeletal muscle [20]. Interestingly, several studies have indicated that the circulating NP concentrations are reduced in human obesity and T2D [21-23]. The latter findings, together with evidence that reduced systemic NP concentrations increase the risk of developing T2D [24, 25], highlight the importance of NP in metabolic disease. Of the NP family, atrial natriuretic peptide (ANP) has been shown to be the most potent stimulator of human AT lipolysis [26], via guanylyl cyclase-coupled natriuretic peptide receptor type A (NPRA)-mediated activation of hormone-sensitive lipase (HSL) [27, 28]. Interestingly, we have recently found that maximal ANP responsiveness is impaired in isolated abdominal subcutaneous adipocytes of obese non-diabetic and T2D men [29]. In line, Rydén and colleagues [30] have recently shown a blunted lipolytic effect of ANP in isolated abdominal subcutaneous adipocytes of obese women and in situ (microdialysis) in abdominal SCAT of overweight men under resting conditions. Importantly, however, the physiological role of exercise-induced ANP-mediated lipolysis in human obesity remains to be established.

It has been shown that endurance exercise training can partly improve $\beta$ adrenoceptor activity, reduce anti-lipolytic $\alpha_{2}$-adrenoceptor sensitivity in human SCAT [31-33], and alleviate ANP-mediated lipolysis in subcutaneous adipocytes in young, metabolically healthy overweight individuals [28,34]. However, to date, it remains elusive if endurance and resistance exercise intervention improves ANPinduced activation of lipolysis in metabolically compromised conditions. 
The aim of the present study was to compare the effect of local combined $\alpha$ - and $\beta$ adrenoceptor blockade on local SCAT lipolysis at rest, during low-intensity endurance-type exercise and during recovery from exercise in middle-aged obese insulin sensitive (IS), obese insulin resistant (IR) and age-matched lean IS men. In addition, we investigated whether a 12-week endurance and resistance exercise training improved the metabolic profile in obese men and (non-)adrenergicallymediated abdominal SCAT lipolysis in obese IR men.

\section{METHODS}

\section{Subjects}

Ten middle-aged healthy lean insulin sensitive (IS), 10 obese IS and 10 obese insulin resistant (IR) men, matched for age and BMI (obese groups) participated in the present study. Subjects were included when they had a stable body weight for at least 3 months prior to the start of the intervention and had no contraindications for participation in an exercise training intervention based on their medical history. Major exclusion criteria were a history, or clinical symptoms, of heart, lung or kidney disease, presence of endocrine anomalies and/or the use of beta-blockers, glucose or lipid-lowering medication. Insulin sensitivity was assessed via homeostasis assessment of insulin resistance (HOMA-IR) [35]. Subjects were classified as insulin sensitive or insulin resistant when HOMA-IR was $\leq 2.3$ [36] or $\geq$ 3.8 [37], respectively. Height, weight, waist and hip circumference and blood pressure were measured during screening. Body composition was measured using a Dual Energy X-ray Absorptiometry scan (Hologic Series Delphi-A Fan Beam Xray Bone Densitometer). One week before the investigational protocol, peak oxygen uptake $\left(\mathrm{VO}_{2 \text { peak }}\right)$ was determined during a maximal cardiopulmonary exercise test performed on an electrical braked cycle ergometer (Gymna Ergofit Cycle 400, Bilzen, Belgium) by using an incremental procedure (work rate increased by $15 \mathrm{~W} / \mathrm{min}$ until volitional exhaustion). Heart rate (electrocardiography) was monitored continuously and $\mathrm{VO}_{2 \text { peak }}$ was measured using a Metalyzer II (Cortex Medical, Leipzig, Germany). The study was approved by the Medical Ethical Committee of the Jessa Hospital and Hasselt University, Hasselt, Belgium, and performed in accordance with the declaration of Helsinki (2008). All individuals gave written informed consent prior to the start of the study.

\section{Experimental protocol}

Subjects arrived at the hospital at 07:30 AM after an overnight fast. They were instructed to consume a standardized meal and snack the evening before the test day (total energy: $2628 \mathrm{~kJ}$ (626 kcal); $23.4 \mathrm{~g}$ fat (10.4g saturated fat); $73.8 \mathrm{~g}$ carbohydrates (of which $6.8 \mathrm{~g}$ sugar); $28.8 \mathrm{~g}$ protein; $2.9 \mathrm{~g}$ salt; $2.3 \mathrm{~g}$ fibres) and to abstain from exhausting activities 48 hours prior to the experimental protocol. On arrival, a catheter was inserted into the antecubital vein for blood sampling. Two microdialysis catheters (CMA 63, CMA Microdialysis AB, Stockholm, Sweden) were inserted percutaneously into SCAT after epidermal anesthesia (EMLA ${ }^{\circledR}$ crème: lidocaine $2.5 \%$ and prilocaine $2.5 \%$, AstraZeneca $A B$ ) at a distance of $6-8$ 
$\mathrm{cm}$ from the umbilicus (one probe on the left side and one probe on the right side of the umbilicus). The probes were connected to a microinfusion pump (Harvard apparatus, Plato BV, Diemen, The Netherlands) and perfused with Ringer solution (in mmol/l: 147 sodium, 4 potassium, 2.25 calcium and 156 chloride; Fresenius Kabi BV, 's Hertogenbosch, The Netherlands) at a perfusion rate of $2.0 \mu \mathrm{l} / \mathrm{min}$. Ethanol $(50 \mathrm{mmol} / \mathrm{l})$ was added to the perfusate to semi-qualitatively estimate changes in local adipose tissue blood flow (ATBF), using the ethanol outflow/inflow (out/in) ratio [38]. A higher ethanol out/in ratio, corresponding to a lower ethanol wash-out, reflects a lower regional ATBF.

One microdialysis catheter was perfused with Ringer solution (control), while the contralateral catheter was perfused with Ringer, supplemented with $100 \mu \mathrm{mol} / \mathrm{l}$ phentolamine $\left(\alpha_{1,2}\right.$-adrenergic receptor antagonist) (Regitin $10 \mathrm{mg} / \mathrm{ml}$; Novartis Pharma BV, The Netherlands) and $100 \mu \mathrm{mol} / \mathrm{l}$ propranolol (nonselective $\beta$ adrenergic receptor antagonist) (propranolol hydrochloride, Dociton $1 \mathrm{mg} / \mathrm{ml}$, Mibe $\mathrm{GmbH}$, Germany), concentrations that completely suppress lipolysis [28, 39, 40]. After a 60-min equilibration period (recovery from insertion), two 30-min fraction of dialysate were collected at a flow rate of $0.3 \mu \mathrm{L} / \mathrm{min}$ after which the perfusion rate was increased to $2.0 \mu \mathrm{L} / \mathrm{min}$ for the remaining of the experiment. During the resting phase, three 15-min fractions of the outgoing dialysate were collected from both sites to determine the extracellular glycerol concentration (reflecting basal lipolysis). Next, subjects performed a single bout of endurance exercise for $60 \mathrm{~min}$ at $40 \%$ of their $\mathrm{VO}_{2 \max }$ on a cycle ergometer while heart rate was monitored continuously (Polar, Kempele, Finland). Exercise was followed by a 60-min recovery period in supine position. During exercise and recovery, dialysate samples were collected at $15 \mathrm{~min}$ intervals without disconnecting the microdialysis probes from the microinfusion pumps.

Ethanol concentrations were determined both in the ingoing (perfusate) and outgoing (dialysate) fluid to assess the ethanol out/in ratio as an indicator of local nutritive blood flow. Ethanol concentrations were determined at the same day, whereas dialysate samples for measurement of extracellular glycerol, glucose and lactate concentrations were immediately frozen and stored at $-80^{\circ} \mathrm{C}$ until analysis. Venous blood samples were taken at rest, during exercise and recovery in prechilled $20 \mathrm{~mL}$ tubes at $15 \mathrm{~min}$ intervals throughout the study protocol.

\section{Indirect calorimetry}

Substrate utilization and energy expenditure were determined at rest and during submaximal exercise via indirect calorimetry using a Metalyzer II (Cortex Medical, Leipzig, Germany). Substrate oxidation rates $(\mathrm{g} / \mathrm{min})$ and energy expenditure were calculated from $\mathrm{VO}_{2}$ and $\mathrm{VCO}_{2}[41,42]$. Water intake was allowed ad libitum during the exercise and recovery period.

\section{Exercise training protocol}

Obese IS and IR subjects participated in a supervised, exercise training program for 12 weeks ( 3 sessions per week) [43]. Subjects were asked not to change their habitual diet during the intervention period. Each training session started with cycling (Excite Bike, Technogym, Zaventem, Belgium) for $45 \mathrm{~min}$ at $65 \% \mathrm{VO}_{2 \text { peak }}$ (heart rate based) from which mean heart rate, mean workload and total energy 
expenditure (calories) were collected. Next, resistance exercises of 5 large muscle groups were performed at $65-70 \%$ of 1 RM (leg press, leg curl, leg extension, vertical traction, arm curl and chest press; Technogym). Training volume and load were gradually increased during the intervention whereby resistance training was increased every 3 weeks. Training sessions were supervised to assure compliance and safety of the participants. After the 12 weeks of exercise training the experimental protocol was repeated and venous blood samples were taken at rest, during exercise and recovery at $15 \mathrm{~min}$ intervals. In addition, SCAT microdialysis was performed in obese IR subjects, as described above.

\section{Biochemical analysis}

Microdialysate samples were analyzed for glycerol, glucose and lactate concentrations by means of bioluminescence on an ISCUS clinical microdialysis analyzer (M dialysis AB, Stockholm, Sweden). Ethanol concentrations in dialysate (out) and perfusate (in) were measured spectrophotometrically using a COBAS FARA semi-automatic analyzer (Roche Diagnostics, Basal, Switzerland) and using a standard ethanol assay kit (Boehringer Mannheim, Germany).

Blood samples were centrifuged at $4^{\circ} \mathrm{C}$ for $10 \mathrm{~min}$ at $1200 \mathrm{~g}$ and plasma and serum was stored at $-80^{\circ} \mathrm{C}$ until further analysis. Plasma free glycerol was measured after precipitation with an enzymatic assay (Enzytec ${ }^{\mathrm{TM}}$ Glycerol, Roche Biopharm, Switzerland), automated on a Cobas Fara spectrophotometric autoanalyzer (Roche Diagnostics, Basel, Switzerland). Plasma FFA, glucose and lactate concentrations were measured with enzymatic assays on an automated spectrophotometer (ABX Pentra 400 autoanalyzer, Horiba ABX, Montpellier, France). Plasma ANP concentrations were measured using an enzyme immunoassay (RayBiotech, Norcross GA, USA). Catecholamine concentrations (adrenalin and noradrenalin) were determined using high performance liquid chromatography with electrochemical detection (ClinRep®) Complete Kit for Catecholamines in Plasma, RECIPE chemicals \& Instruments $\mathrm{GmbH}$, Munich, Germany). Serum insulin concentrations were determined with radioimmunoassay kits (Human Insulin specific RIA Kit, Millipore Corporation, MA, USA).

\section{Statistical analysis}

All data are expressed as means \pm SEM. Normal distribution was tested by the Kolmogorov-Smirnov test. Subjects were excluded from analyses when dialysate samples of 2 subsequent time points were missing, in order to maintain paired samples. Dialysate and systemic exercise responses were expressed as the area under the curve (AUC) and the incremental area under the curve (iAUC), calculated by the trapezoid method. Cross-sectional analyses (differences between groups and conditions) for the microdialysis lipolysis data were analyzed with a two-way repeated-measures ANOVA. In case of significance, post-hoc analyses with Bonferroni correction were applied to identify significant within-group effects. Differences in plasma concentrations and substrate metabolism between groups were tested with a one-way ANOVA and differences within groups were analyzed by means of paired $t$-test. Intervention effects in the obese groups were analyzed with a two-way repeated-measures ANOVA (with pre- and post-intervention as conditions), with Bonferroni post-hoc correction to detect within-group effects. 
Three subjects dropped out of the exercise intervention, due to medical $(n=1)$ or motivational reasons $(n=2)$ and were therefore excluded from the intervention (pre vs. post) analyses. SPSS 21 for Macintosh OS X was used to perform all calculations (IBM Corporation, Armonk, NY, USA). The level of statistical significance was set at $p<0.05$ (2-tailed), while $p<0.10$ was considered a tendency.

\section{RESULTS}

\section{BASELINE}

\section{Anthropometric and clinical characteristics}

Subjects' characteristics are presented in Table 1. By design, there was a significant difference between the lean and the obese IS and/or obese IR group with respect to body weight, $\mathrm{BMI}$, WH-ratio, whole-body fat percentage, android (i.e. trunk region) and gynoid (including hip and leg regions) fat mass (all $p<0.05$ ). Furthermore, HOMA-IR and fasting serum insulin concentrations were significantly higher in obese IR compared to the lean and obese IS individuals $(p<0.001$ for both parameters in both groups). Obese IS and obese IR individuals only differed in android fat mass, which was higher in the obese IR group $(p=0.017)$ (Table 1). With respect to physical fitness, $\mathrm{VO}_{2 \text { peak/FFM }}$ and $\mathrm{W}_{\text {peak/FFM }}$ were significantly lower in the obese IS and obese IR group as compared to the lean group $(p<0.01$ and $p<0.001$, respectively) (Table 1 ), while maximal heart rate and maximal respiratory quotient $(\mathrm{RQ})$ were comparable between groups. 
Table 1. Characteristics of obese insulin sensitive, obese insulin resistant individuals and healthy lean controls

\begin{tabular}{|c|c|c|c|c|}
\hline \multicolumn{5}{|c|}{ 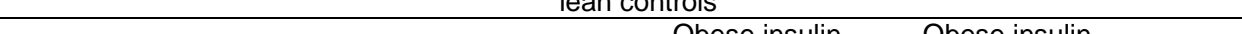 } \\
\hline+2 & $\begin{array}{c}\text { Lean } \\
(n=10) \\
\end{array}$ & $\begin{array}{c}\text { Obese insulin } \\
\text { sensitive } \\
(n=10)\end{array}$ & $\begin{array}{c}\text { Obese insulin } \\
\text { resistant } \\
(n=10)\end{array}$ & $P_{\text {ANOVA }}$ \\
\hline Age, years & $45 \pm 2$ & $47 \pm 2$ & $43 \pm 1$ & 0.527 \\
\hline \multicolumn{5}{|l|}{ Cardiometabolic risk } \\
\hline $\begin{array}{l}\overline{\text { Fasting plasma glucose }} \\
\text { (mmol/L) }\end{array}$ & $5.5 \pm 0.0$ & $5.2 \pm 0.1$ & $5.8 \pm 0.2 \dagger$ & 0.050 \\
\hline $\begin{array}{l}\text { Fasting serum insulin }(\mathrm{mU} / \mathrm{L}) \\
\text { HOMA-IR } \\
\text { Systolic BP }(\mathrm{mmHg}) \\
\text { Diastolic BP }(\mathrm{mmHg})\end{array}$ & $\begin{array}{c}7.3 \pm 0.6 \\
1.8 \pm 0.1 \\
122 \pm 2 \\
72 \pm 1\end{array}$ & $\begin{array}{c}9.2 \pm 0.6 \\
2.1 \pm 0.1 \\
135 \pm 6 \\
81 \pm 4\end{array}$ & $\begin{array}{c}19.6 \pm 1.6^{* \star *} \dagger \\
5.0 \pm 0.4^{* \star *} \dagger \\
143 \pm 6 \\
86 \pm 5\end{array}$ & $\begin{array}{l}<0.001 \\
<0.001 \\
0.066 \\
0.108\end{array}$ \\
\hline \multicolumn{5}{|l|}{ Body composition } \\
\hline $\begin{array}{l}\text { Body weight }(\mathrm{kg}) \\
\text { Body mass index }\left(\mathrm{kg} / \mathrm{m}^{2}\right) \\
\text { Waist-to-hip ratio } \\
\text { Fat mass }(\mathrm{kg}) \\
\text { Fat percentage }(\%) \\
\text { Fat free mass }(\mathrm{kg})\end{array}$ & $\begin{array}{c}79.9 \pm 2.9 \\
23.7 \pm 0.4 \\
1.00 \pm 0.00 \\
16.4 \pm 1.1 \\
22.0 \pm 0.8 \\
57.4 \pm 1.8\end{array}$ & $\begin{array}{c}101.5 \pm 3.2^{* *} \\
32.6 \pm 0.4^{* * *} \\
1.04 \pm 0.01^{*} \\
30.0 \pm 1.7^{* *} \\
31.4 \pm 0.9^{* * *} \\
65.0 \pm 1.5\end{array}$ & $\begin{array}{c}109.6 \pm 4.7^{* * *} \\
33.9 \pm 0.7^{* \star *} \\
1.05 \pm 0.01^{*} \\
34.2 \pm 1.8^{* \star *} \\
33.2 \pm 1.1^{* \star *} \\
68.4 \pm 2.8^{* *}\end{array}$ & $\begin{array}{c}<0.001 \\
<0.001 \\
0.010 \\
<0.001 \\
<0.001 \\
0.004\end{array}$ \\
\hline $\begin{array}{l}\text { Exercise capacity } \\
\text { VO2 peak }\left(\mathrm{m}^{\star} \mathrm{min}^{-1} \mathrm{~kg}^{-1}(\mathrm{FFM})\right) \\
\text { Wmax }\left(\mathrm{Watt}^{*} \mathrm{~kg}^{-1}(\mathrm{FFM})\right)\end{array}$ & $\begin{array}{c}62 \pm 3 \\
4.9 \pm 0.1 \\
\end{array}$ & $\begin{array}{c}48 \pm 2^{* *} \\
3.7 \pm 0.2^{* * *}\end{array}$ & $\begin{array}{c}48 \pm 1^{\text {** }} \\
3.4 \pm 0.1^{\text {*** }}\end{array}$ & $\begin{array}{r}0.002 \\
<0.001 \\
\end{array}$ \\
\hline
\end{tabular}

Data are mean \pm SE. ${ }^{*}$ Significantly different from lean group $p<0.05 ;{ }^{* *} p<0.01 ;{ }^{* * *} p<0.001$.

† Significantly different from obese insulin sensitive group $(p<0.05)$. FFM: fat free mass; HR: heart rate; $\mathrm{RER}$ : respiratory exchange ratio; $\mathrm{VO}_{2}$ peak: maximum oxygen uptake; Wmax: maximum power output

\section{Systemic responses during rest, exercise and recovery phase}

Under resting conditions, plasma glycerol, FFA, glucose, lactate, ANP, adrenalin and noradrenalin concentrations were comparable between groups, while fasting serum insulin concentration was higher in obese IR compared to lean and obese IS individuals (Figure $1 \mathrm{~A}-\mathrm{H}$ ). During exercise, plasma concentrations of glycerol, FFA, glucose, ANP, adrenalin and noradrenalin increased to the same extent in all groups (Figure 1). Exercise increased plasma lactate concentrations in all groups, which was most pronounced in obese IR as compared to lean individuals $\left(p_{A N O V A}=0.044\right)$ (Figure $\left.1 \mathrm{D}\right)$. The exercise-induced increase in plasma ANP was similar in all groups $\left(p_{A N O V A}=0.300\right)$, but peak plasma ANP concentrations were reached earlier during exercise in the lean compared to the obese groups $(p=0.034)$ (Figure $1 \mathrm{E})$. Serum insulin levels were significantly higher during exercise in obese IR as compared to obese IS and lean individuals, with no differences between the latter two groups (Figure $1 \mathrm{~F}$ ). During exercise, the increase in plasma adrenalin and noradrenalin concentrations was comparable between groups (Figure $1 \mathrm{G}-\mathrm{H}$ ). During recovery, plasma glycerol, glucose, adrenalin, noradrenalin and ANP concentrations decreased back to baseline concentrations (Figure 1), while plasma lactate concentrations tended to remain elevated in the obese IR group ( $p_{\text {ANOVA }}=0.061$ ) (Figure $\left.1 \mathrm{D}\right)$. Furthermore, plasma FFA concentrations peaked in the first 15 minutes of the recovery period and 
remained significantly elevated in obese $I R$ as compared to lean individuals $\left(\mathrm{p}_{\mathrm{ANOVA}}=0.020\right)$ (Figure $\left.1 \mathrm{~B}\right)$. Serum insulin concentrations remained significantly elevated during recovery in the obese IR group compared to the obese IS and lean group (both $p_{\text {ANOva }}<0.001$ ), with no differences between the latter two groups (Figure $1 \mathrm{~F}$ ). Detailed systemic plasma responses during baseline, exercise and recovery are shown in Supplementary Table S1.

A

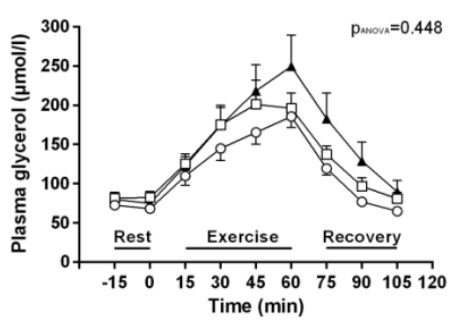

C

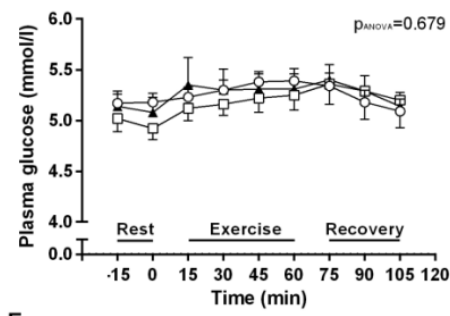

E

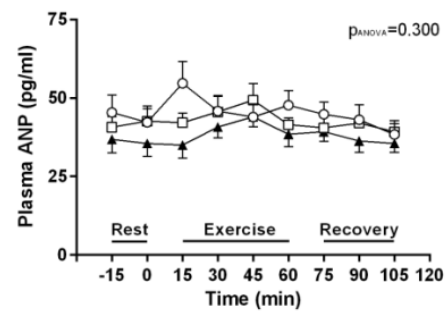

G

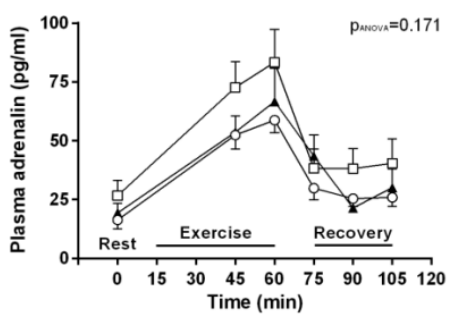

B

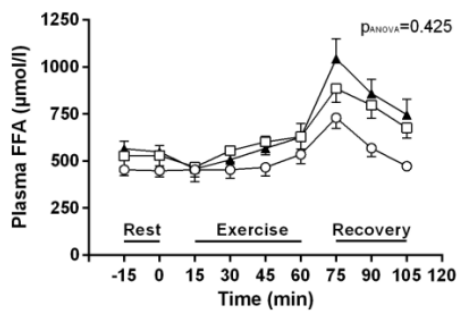

D
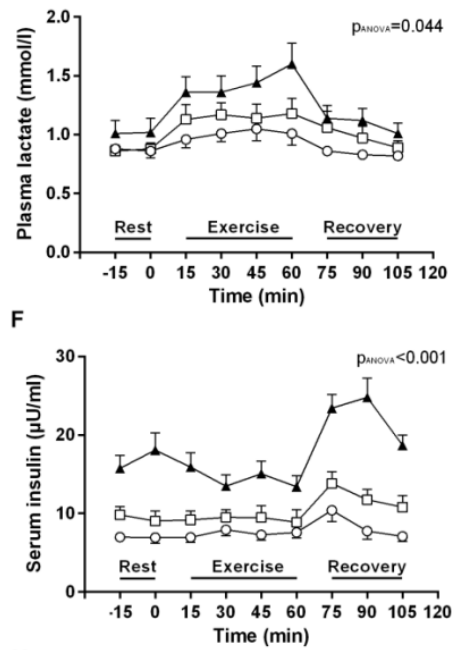

H

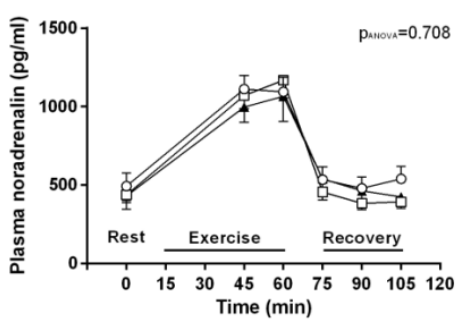

Figure 1. Plasma glycerol, FFA, glucose, lactate, ANP, adrenalin, noradrenalin and serum insulin concentrations at rest, during exercise and recovery.

Systemic glycerol (A), FFA (B), glucose (C), lactate (D), ANP (E), insulin (F), adrenalin (G) and noradrenalin $(\mathbf{H})$ responses in lean (white circles), obese insulin sensitive (white squares) and obese insulin resistant (black triangles) individuals. Data are presented as mean $\pm S E M$. P $P_{\text {ANova }}$ values represent differences in exercise-induced systemic responses between groups. 
Substrate oxidation and energy expenditure

Substrate oxidation and energy expenditure were determined at rest and during exercise. Whole-body energy expenditure $(\mathrm{kJ} / \mathrm{min}), R Q$, carbohydrate and fat oxidation (as percentage of energy expenditure) were not different between groups at rest, nor during exercise (Supplementary Figure $1 \mathrm{~A}-\mathrm{E}$ ).

\section{Microdialysis}

Abdominal subcutaneous adipose tissue blood flow

At rest, lean individuals had a significantly lower ethanol out/in ratio, reflecting a higher ATBF, compared to both obese groups $(p<0.01)$, whilst no significant difference in ATBF was observed between both obese groups (Figure $2 \mathrm{~A}, \mathrm{C}$ and $E)$. Local $\alpha$-/ $\beta$-adrenergic blockade induced a significant increase in ethanol out/in ratio in the lean group (Figure $2 A$ ), reflecting a reduced ATBF, while this effect disappeared during exercise. Moreover, this adrenergic sensitivity of ATBF was not observed in the obese IS or IR group (Figure $2 \mathrm{C}$ and E). Exercise induced a decrease in ethanol out/in ratio, reflecting an increase in ATBF, in all groups. This exercise-induced increase in ATBF tended to be higher in lean as compared to obese IS and obese IR individuals ( $p=0.093, p=0.087$, respectively) (Figure $2 \mathrm{~A}, \mathrm{C}$ and $E$ ). During recovery, ATBF returned to resting levels, with a significantly higher ATBF (i.e. a lower ethanol out/in ratio) in the lean group compared to both obese groups (Figure $2 \mathrm{~A}, \mathrm{C}$ and $\mathrm{E}$ ). Details with respect to ethanol out/in ratio during baseline, exercise and recovery are shown in Supplementary Table S2. 

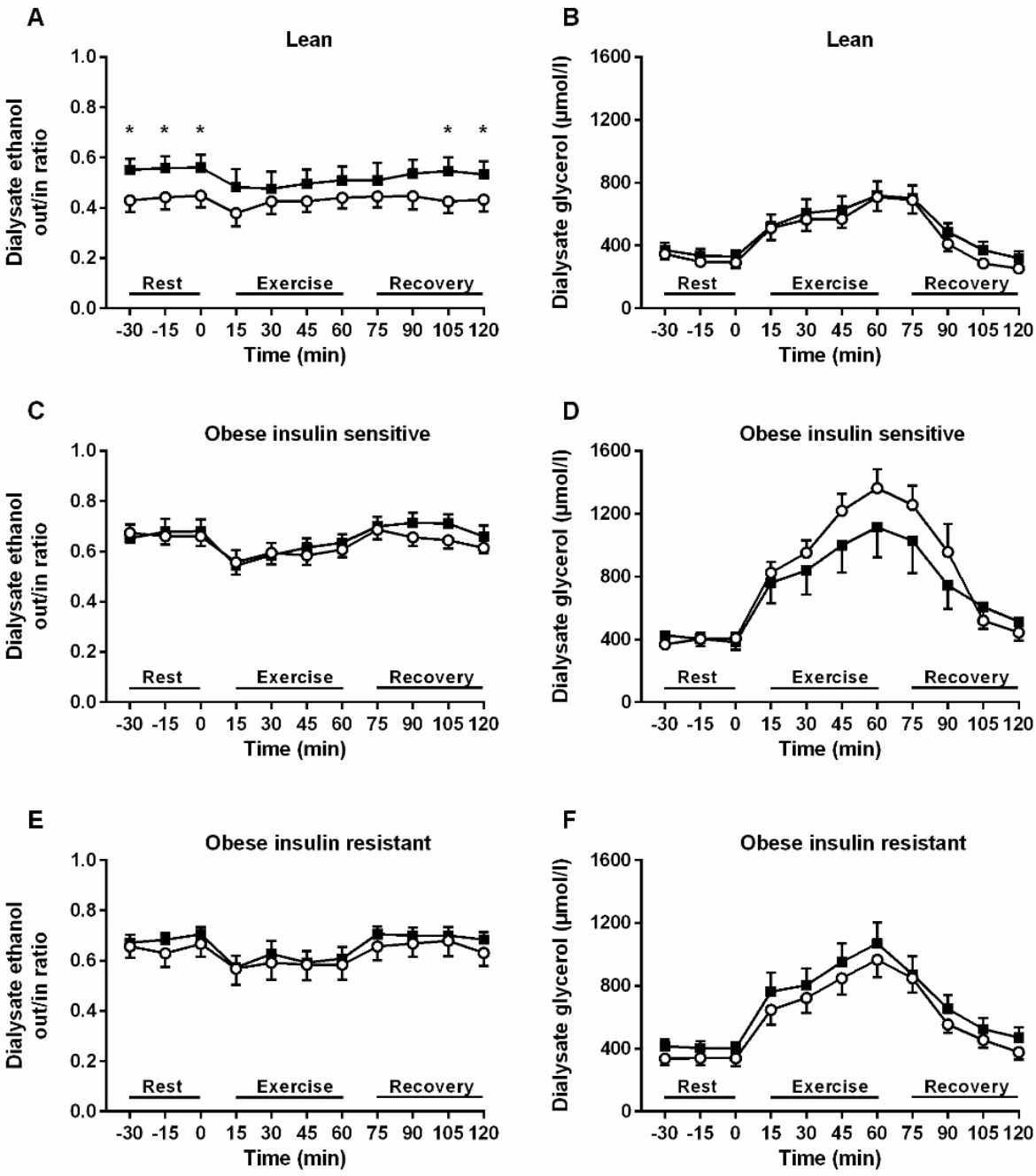

Figure 2. Changes in subcutaneous adipose tissue extracellular glycerol concentration and adipose tissue blood flow indices.

Subcutaneous adipose tissue ethanol ratio's in lean (A), obese insulin sensitive (B) and obese insulin resistant $(\mathbf{C})$ individuals. Changes in extracellular glycerol concentration in lean (D), obese insulin sensitive (E) and obese insulin resistant (F) individuals at rest, during exercise and recovery in control probe (white circles) and the probe perfused with phentolamine and propranolol (black squares). Data are presented as mean \pm SEM. * Significantly different from the control probe $(p<0.05)$. 


\section{Abdominal subcutaneous adipose tissue lipolysis}

In SCAT, resting extracellular glycerol concentrations were comparable between groups (Figure $2 \mathrm{~B}, \mathrm{D}$ and $\mathrm{F}$ ). Local $\alpha-\beta$-adrenergic blockade had no significant effects on resting extracellular SCAT glycerol concentration in either of the groups (Figure $2 \mathrm{~B}, \mathrm{D}$ and $\mathrm{F}$ ). During exercise, extracellular glycerol concentration significantly increased in all groups. In obese IS individuals, exercise-induced increase in glycerol concentration $\left(\mathrm{AUC}_{0-60}\right)$ was higher compared to lean $(p=0.011)$, but not obese IR individuals $(p=0.816)$ ( (Figure $2 B, D$ and $F)$. Local $\alpha$ $/ \beta$-adrenergic blockade induced a significant reduction in the exercise-induced increase in extracellular glycerol in the obese IS group $(p=0.020)$, but not in the lean IS or obese IR group (Figure 3). During recovery, extracellular glycerol concentrations decreased in all groups, but remained significantly elevated in the obese IS as compared to the lean group, with no differences between both obese groups (Figure $2 \mathrm{~B}, \mathrm{D}$ and F). Additionally, there were no significant effects of $\alpha$ - $/ \beta$ adrenergic blockade on the extracellular glycerol concentration during recovery in any group (Figure $2 \mathrm{~B}, \mathrm{D}$ and $\mathrm{F}$ ). Details with respect to dialysate glycerol concentrations at rest, during exercise and recovery are shown in Supplementary Table S2.

Abdominal SCAT extracellular glucose and lactate concentrations and responses were comparable between groups at rest, during exercise and recovery (Supplementary Table S3).

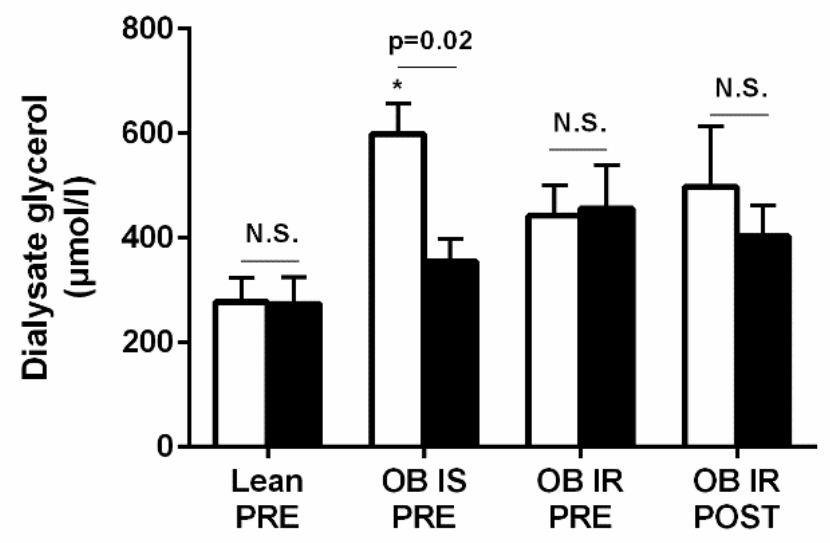

Figure 3. Exercise-induced increase in subcutaneous adipose tissue extracellular glycerol concentration.

Mean changes in subcutaneous adipose tissue extracellular glycerol concentration during $1 \mathrm{~h}$ of lowintense exercise (40\% VO2peak). Extracellular glycerol concentrations are determined in control probe (white bars) and the probe perfused with phentolamine and propranolol (black bars). Changes were calculated by the difference between the mean glycerol concentrations during exercise and the baseline concentration $\left(p_{\text {group }}=0.009, p_{\text {treatment }}=0.069, p_{\text {treatment }}\right.$ group $=0.035$ ). Data are presented as mean $\pm S E M$.

* Significantly $(p<0.05)$ different compared to the control probe from the lean group; N.S.: not significant. 


\section{EXERCISE TRAINING INTERVENTION}

\section{Anthropometry, exercise capacity and systemic responses}

In both obese groups, exercise training led to a significant reduction in body weight, BMI, whole-body fat percentage as well as android and gynoid fat mass (Table 2). Whole-body insulin sensitivity (HOMA-IR) was significantly improved in the obese IR group $\left(p_{\text {time }}=0.005\right)$, but not in the obese IS group. Furthermore, physical fitness $\left(\mathrm{VO}_{\text {2peak/FFM }}\right.$ as well as $\left.\mathrm{W}_{\text {max/FFM }}\right)$ improved significantly following the 12-week exercise training (Table 2).

The training intervention induced a significant reduction in resting plasma FFA (Figure $4 \mathrm{C}$ and $\mathrm{D}$ ) and tended to reduce fasting ANP concentrations in both obese groups (Figure $4 \mathrm{~K}$ and $\mathrm{L}$ ). Resting blood glucose concentration increased in the IS group but not in the IR group (Figure $4 \mathrm{E}$ and F). The training intervention did not induce significant changes in plasma glycerol, insulin, lactate, adrenalin or noradrenalin concentrations. However, resting insulin concentrations, as well as insulin concentration during exercise and recovery remained elevated in the obese IR compared to obese IS group (Figure 4 I-J).

The exercise-induced increase in plasma FFA (Figure $4 \mathrm{C}$ and D), lactate (Figure 4 $\mathrm{G}$ and $\mathrm{H}$ ) and adrenalin (Figure $4 \mathrm{M}$ and $\mathrm{N}$ ) concentrations were significantly blunted after intervention. Peak blood ANP concentrations (Figure $4 \mathrm{~K}$ and L) tended to be reduced in both obese groups. The increase in plasma glycerol, insulin and glucose concentrations during exercise remained unchanged.

In the recovery period, beside reduced plasma FFA (Figure $4 \mathrm{C}$ and $\mathrm{D}$ ) and adrenalin concentrations (Figure $4 \mathrm{M}$ and $\mathrm{N}$ ), no significant training-induced changes were observed in plasma glycerol, glucose, lactate, ANP, noradrenalin or serum insulin concentrations (Figure 4). In addition, the training intervention did not induce changes in whole-body energy expenditure and substrate oxidation (Supplemental Figure $1 \mathrm{~A}-\mathrm{E}$ ). Detailed post-intervention systemic plasma responses at rest, during exercise and recovery are shown in Supplementary Table S4. 

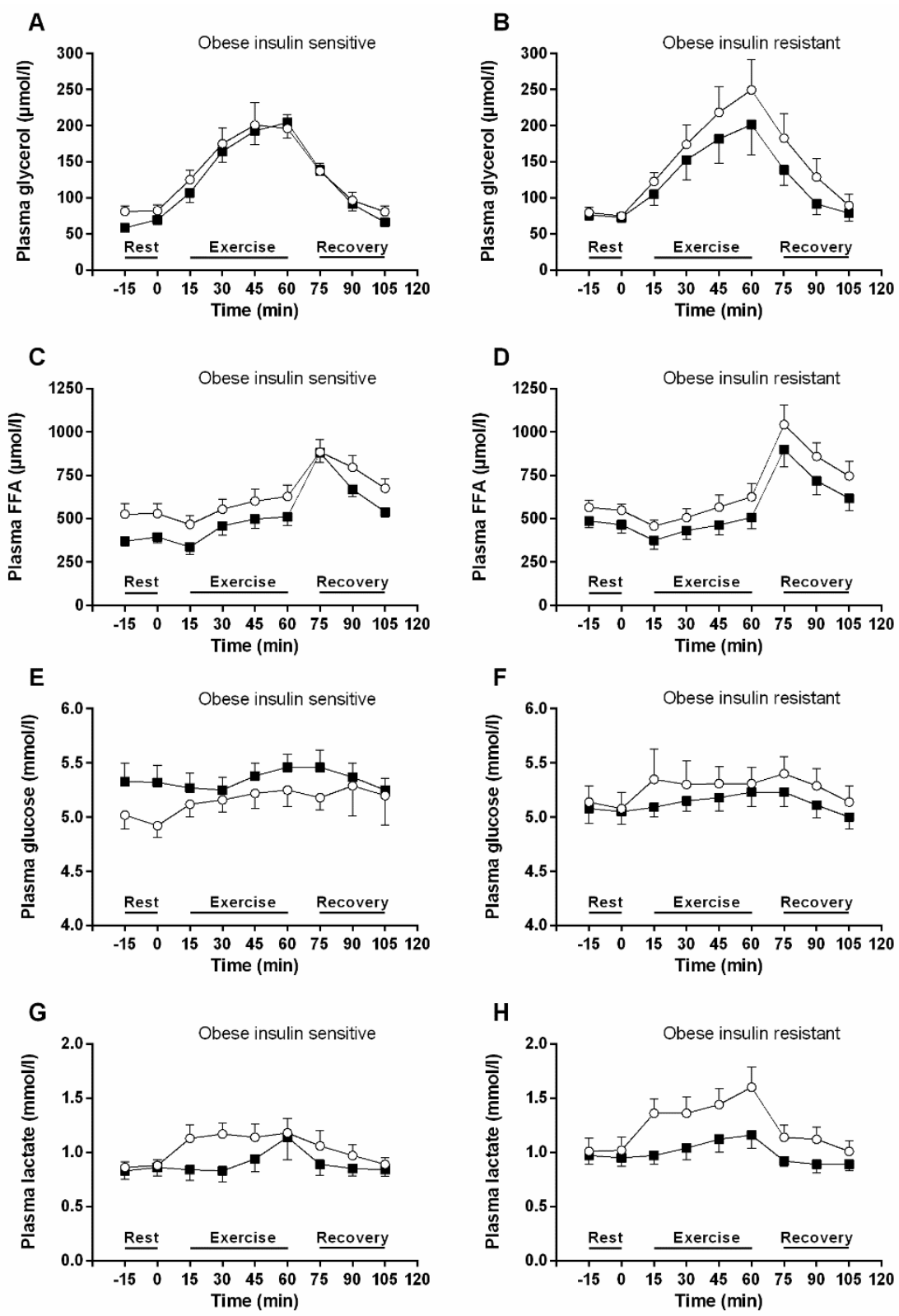

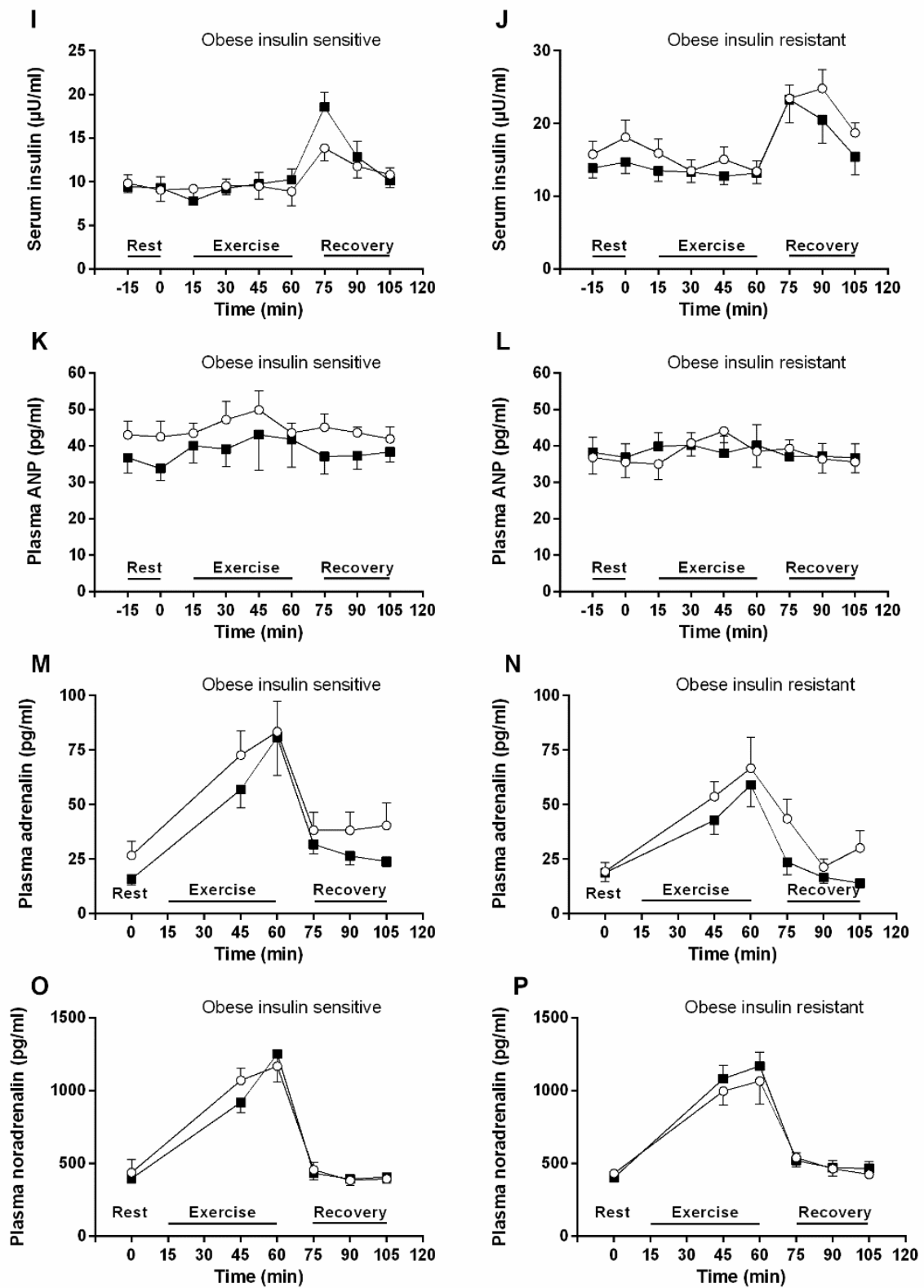

Figure 4. Exercise training-induced changes in systemic plasma glycerol, FFA, glucose, lactate, ANP, adrenalin, noradrenalin and serum insulin in obese individuals.

Systemic glycerol (A-B), FFA (C-D), glucose (E-F), lactate (G-H), insulin (I-J), ANP (K-L), adrenalin (M$\mathbf{N}$ ) and noradrenalin (O-P) of obese insulin sensitive and obese insulin resistant individuals at baseline (white circles) and after 12 weeks of exercise training intervention (black squares). Data are presented as mean \pm SEM. 
Abdominal subcutaneous adipose tissue blood flow and lipolysis

Following exercise intervention, SCAT lipolysis was investigated in the obese IR group only. Ethanol out/in ratio (Figure $5 \mathrm{~A}$ ) as well as resting, exercise-induced and recovery-related extracellular glycerol concentration (Figure $5 \mathrm{~B}$ ) were not altered after exercise intervention. Additionally, $\alpha-/ \beta$-adrenergic blockade had no significant effect on resting, exercise-induced or recovery-related extracellular glycerol concentration in SCAT following the exercise training program (Figure $5 \mathrm{~B}$; Figure 3).

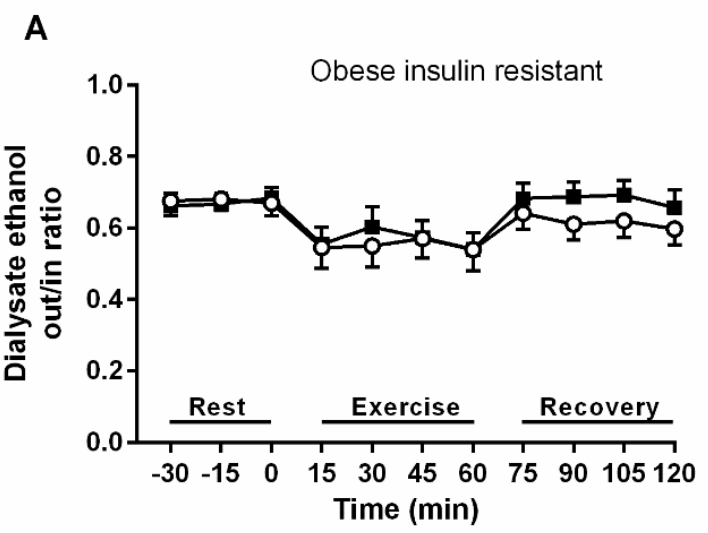

B

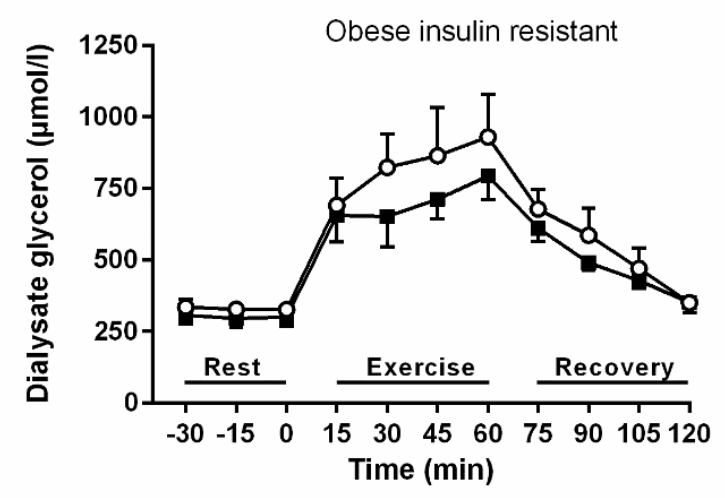

Figure 5. Exercise training-induced changes in subcutaneous adipose tissue extracellular glycerol concentration and adipose tissue blood flow indices in obese insulin resistant individuals.

Subcutaneous adipose tissue ethanol ratio $(\mathbf{A})$ and extracellular glycerol concentration $(\mathbf{B})$ in the obese insulin resistant individuals at rest, during exercise and recovery after 12 weeks of exercise training in control probe (white circles) and the probe perfused with phentolamine and propranolol (black squares). Data are presented as mean \pm SEM. 


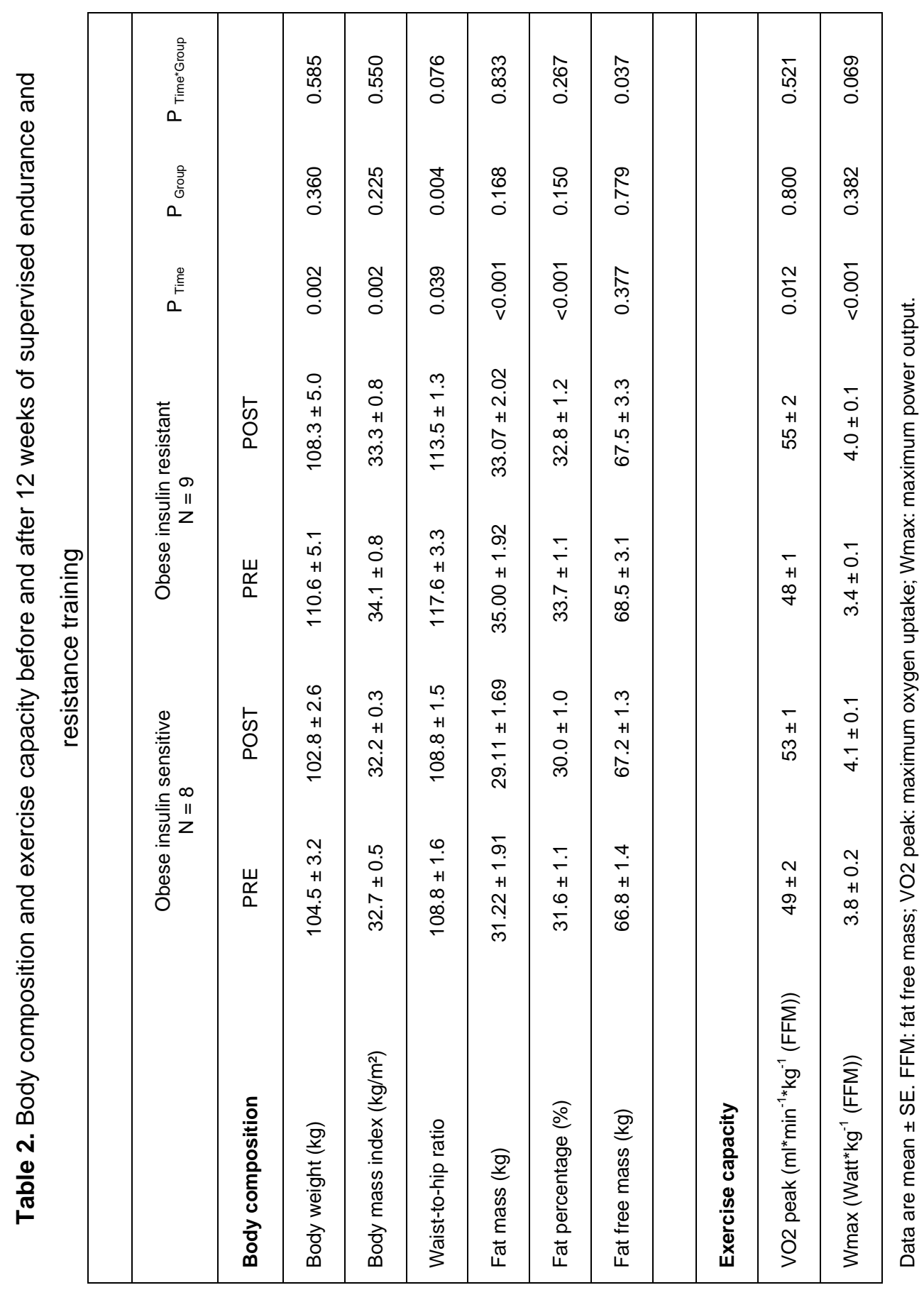




\section{DISCUSSION}

The present study is the first to investigate (non-)adrenergically-mediated lipolysis during low-intensity endurance-type exercise in abdominal subcutaneous adipose tissue (SCAT) in middle-aged obese IS and obese IR men as compared to agematched lean IS men. Furthermore, we examined whether a 12-week exercise intervention altered resting and exercise-induced (non-)adrenergically-mediated SCAT lipolysis in obese IR individuals. Here, we demonstrated that the exerciseinduced increase in abdominal SCAT extracellular glycerol concentration (reflecting local lipolysis) was more pronounced in obese as compared to lean individuals, which may at least partly be explained by the higher adipose tissue blood flow (ATBF) in lean individuals. Exercise-induced SCAT lipolysis was substantially reduced $(\sim 40 \%)$ following local combined $\alpha$-/ $\beta$-adrenergic blockade in obese IS individuals, but not in obese IR or lean IS individuals. Finally, the exercise training intervention improved body composition, physical fitness and exercise-induced systemic responses in both obese groups, and insulin sensitivity in the obese IR group. However, this was not accompanied by changes in adrenergically- and nonadrenergically-mediated lipolysis in the SCAT of obese IR individuals. Collectively, our findings indicate that exercise-induced lipolysis is predominantly mediated by non-adrenergic factors, most likely mediated by natriuretic peptides (NP) in middleaged lean IS, obese IS and obese IR individuals.

The present study showed a more pronounced increase in exercise-mediated SCAT extracellular glycerol concentration in obese IS as compared to lean individuals. Although the lipolytic response in abdominal SCAT is often blunted in human obesity [9], the higher exercise-induced extracellular glycerol levels in both obese groups is likely explained by the substantially lower ATBF in the obese state, which contributes to higher extracellular glycerol concentrations due to a lower removal of glycerol from the AT $[16,44]$. Local $\alpha$-/ $\beta$-adrenergic blockade substantially reduced basal ATBF in lean but not in the obese individuals. This might suggest that adrenergic sensitivity of ATBF is reduced in the obese state, which is in line with previous findings $[39,45]$. Thus, these differences in ATBF between lean and obese individuals should be taken into account when interpreting local SCAT lipolysis.

Interestingly, combined $\alpha$-/ $\beta$-adrenergic receptor blockade reduced exerciseinduced SCAT lipolysis in obese IS men but not in lean and obese IR individuals. In obese IR individuals, the lipolytic activity of the $\beta$-adrenergic receptors is attenuated $[9,11,12]$, while an increase in anti-lipolytic $\alpha_{2}$-adrenergic receptors in SCAT reduce exercise-mediated lipolysis [39]. In addition, obese IR individuals often display lower plasma catecholamine (adrenaline, noradrenaline) responses to physical exercise [46], although the latter was not observed in the current study. Therefore, the reduced exercise-mediated lipolytic response upon combined $\alpha$ - $/ \beta$ adrenergic receptor blockade in the obese IS individuals, as opposed to the obese IR group, might suggest differences in adrenergic receptor expression and sensitivity. The blunted lipolytic response in obese IR individuals might also be explained by the significantly higher fasting and exercise induced serum insulin levels. Since the anti-lipolytic effects of insulin might be normal or only slightly impaired in obese AT [47-49], the observed hyperinsulinemia in the obese IR group might have contributed to the attenuated adrenergically-mediated SCAT lipolysis 
as compared to the obese IS group, as previously shown [50]. In addition, increased plasma lactate concentrations in obese IR individuals, may have contributed to the reduced lipolytic response, since lactate has been shown to inhibit lipolysis in mice [51] and human primary adipocytes in vitro [18].

The present study implies that non-adrenergic regulators of lipolysis play a major role during low-intensity endurance-type exercise. It has previously been demonstrated that propranolol [28] and phentolamine [39, 40] fully inhibit adrenergically-mediated SCAT lipolysis at the concentrations used in the present study. The exercise-induced lipolytic response was only suppressed to a minor extent in all groups, clearly indicating a major contribution of non-adrenergic components to SCAT lipolysis during exercise. Importantly, other (anti-)lipolytic factors, such as the parathyroid hormone, cortisol and growth hormone are less important during the type and duration of exercise as applied in the current study $[7,16]$. Noteworthy, atrial natriuretic peptide (ANP) may be responsible for the exercise-induced increase in SCAT lipolysis in the present study, especially since ANP is one of the major lipolytic hormones produced upon exercise [26, 52], next to sympathetic nervous system activation. In line with our findings, it has previously been shown that non-adrenergically mechanisms are involved in SCAT lipolysis, accounting for $\sim 65 \%$ of the exercise-mediated lipolysis in young healthy lean and overweight men $[15,16]$. Previously, a lower in vivo ANP responsiveness in SCAT of overweight (31) and obese (30) individuals was observed. In line, the nonadrenergically regulation of SCAT lipolysis has been reported to be more pronounced in young healthy overweight as compared to lean men [16], suggesting that ANP-mediated SCAT lipolysis is particularly important in the overweight and obese state. Interestingly, in contrast to obese IS individuals, we found that SCAT lipolysis in obese IR men was not affected by local $\alpha-\beta$ adrenergic blockade, which may propose an interaction between SCAT adrenergically-mediated lipolysis and whole-body insulin resistance. These findings support a catecholamine-resistance phenotype of the SCAT during exercise especially in the obese IR state. Therefore, a major role for ANP in SCAT lipolysis during exercise can be suggested, which is sustained in the obese IR state.

To ensure adequate fatty acid delivery to the working skeletal muscles during exercise, the sustained ANP-mediated lipolytic response, the adrenergicallymediated lipolysis and a blunted insulin- and lactate-mediated inhibition of lipolysis, might have contributed to the more pronounced SCAT lipolysis as observed in the obese IS men. Because most of the anti-lipolytic action of insulin is mediated through stimulation and activation of cellular phosphodiesterase-3B [53, 54], which degrades CAMP, attenuation of the ANP-related SCAT lipolysis by insulin is unlikely, since the latter is cGMP-mediated [27]. Likewise, it has been shown that NP receptor expression (both functional NPRA and scavenging NPRC) in SCAT associates with whole-body insulin sensitivity [55].

The 12-week exercise intervention increased insulin sensitivity in the obese IR group. While plasma glycerol, FFA, lactate, ANP and adrenalin concentrations were significantly reduced after the intervention, only minor reductions were observed for circulating glucose, insulin and noradrenalin concentrations. However, local exercise-induced abdominal SCAT lipolysis was not improved following the exercise intervention. Moreover, the efficiency of local $\alpha-\beta$-adrenergic blockade was not affected by the exercise intervention. Together, these data suggest that 
even after a substantial improvement in metabolic profile (as indicated by our systemic responses) and body composition after a 12-week exercise intervention, lipolytic disturbances remain unaffected in SCAT of obese IR individuals. Optimized therapies are warranted to achieve enhancements in the regulation of SCAT lipolysis, especially in metabolically compromised individuals.

Although evidence suggests that exercise training induces beneficial changes in SCAT insulin sensitivity [56], SCAT adrenergic sensitivity [56-58] as well as ANPmediated lipolysis [59], future mechanistic studies are needed to obtain a better understanding of the hormonal (exercise-induced) lipolytic regulation in lean and obese individuals with a different degree of insulin sensitivity. Unfortunately, a NPRA receptor agonist/antagonist for use in humans is currently unavailable, which hampers strong conclusions about the physiological role of ANP in human AT lipolysis in vivo.

In conclusion, this study is the first to report the integrated physiological role of adrenergically- and non-adrenergically-mediated SCAT lipolysis during lowintensity endurance-type exercise in middle-aged lean IS, obese IS and obese IR men, and the effects of a 12-week supervised exercise intervention on these processes in obese individuals. The present data demonstrate a major role for nonadrenergically regulated lipolysis in SCAT during low-intensity exercise, likely involving ANP-mediated lipolysis. Furthermore, the exercise training intervention was not accompanied by changes in SCAT lipolysis in obese IR individuals, regardless of improvements in metabolic profile and body composition. 


\section{REFERENCES}

1. Goossens $\mathrm{GH}$. The role of adipose tissue dysfunction in the pathogenesis of obesityrelated insulin resistance. Physiol Behav. 2008;94(2):206-18.

2. Lafontan M. Adipose tissue and adipocyte dysregulation. Diabetes Metab. 2014;40(1):16-28.

3. Stinkens R, Goossens GH, Jocken JW, Blaak EE. Targeting fatty acid metabolism to improve glucose metabolism. Obes Rev. 2015;16(9):715-57.

4. Frayn KN. Adipose tissue as a buffer for daily lipid flux. Diabetologia. 2002;45(9):1201-10.

5. Jocken JW, Blaak EE. Catecholamine-induced lipolysis in adipose tissue and skeletal muscle in obesity. Physiol Behav. 2008;94(2):219-30.

6. Fruhbeck G, Mendez-Gimenez L, Fernandez-Formoso JA, Fernandez S, Rodriguez A. Regulation of adipocyte lipolysis. Nutr Res Rev. 2014;27(1):63-93.

7. Hansen D, Meeusen R, Mullens A, Dendale P. Effect of acute endurance and resistance exercise on endocrine hormones directly related to lipolysis and skeletal muscle protein synthesis in adult individuals with obesity. Sports Med. 2012;42(5):415-31.

8. Ryden $M$, Jocken J, van Harmelen V, Dicker A, Hoffstedt J, Wiren $M$, et al. Comparative studies of the role of hormone-sensitive lipase and adipose triglyceride lipase in human fat cell lipolysis. Am J Physiol Endocrinol Metab. 2007;292(6):E184755.

9. Jocken JW, Goossens GH, van Hees AM, Frayn KN, van Baak M, Stegen J, et al. Effect of beta-adrenergic stimulation on whole-body and abdominal subcutaneous adipose tissue lipolysis in lean and obese men. Diabetologia. 2008;51(2):320-7.

10. Mauriege P, Despres JP, Prud'homme D, Pouliot MC, Marcotte M, Tremblay A, et al. Regional variation in adipose tissue lipolysis in lean and obese men. $J$ Lipid Res. 1991;32(10):1625-33.

11. Reynisdottir S, Wahrenberg H, Carlstrom K, Rossner S, Arner P. Catecholamine resistance in fat cells of women with upper-body obesity due to decreased expression of beta 2-adrenoceptors. Diabetologia. 1994;37(4):428-35.

12. Lafontan $M$, Berlan $M$. Fat cell adrenergic receptors and the control of white and brown fat cell function. J Lipid Res. 1993;34(7):1057-91.

13. Arner $P$, Kriegholm E, Engfeldt $P$, Bolinder J. Adrenergic regulation of lipolysis in situ at rest and during exercise. J Clin Invest. 1990;85(3):893-8.

14. Hellstrom L, Blaak E, Hagstrom-Toft E. Gender differences in adrenergic regulation of lipid mobilization during exercise. Int J Sports Med. 1996;17(6):439-47.

15. Moro C, Polak J, Hejnova J, Klimcakova E, Crampes F, Stich V, et al. Atrial natriuretic peptide stimulates lipid mobilization during repeated bouts of endurance exercise. Am J Physiol Endocrinol Metab. 2006;290(5):E864-9.

16. Moro C, Pillard F, de Glisezinski I, Klimcakova E, Crampes F, Thalamas C, et al. Exercise-induced lipid mobilization in subcutaneous adipose tissue is mainly related to natriuretic peptides in overweight men. Am $\mathrm{J}$ Physiol Endocrinol Metab. 2008;295(2):E505-13.

17. Lafontan M. Fat cells: afferent and efferent messages define new approaches to treat obesity. Annu Rev Pharmacol Toxicol. 2005;45:119-46.

18. Liu C, Wu J, Zhu J, Kuei C, Yu J, Shelton J, et al. Lactate inhibits lipolysis in fat cells through activation of an orphan G-protein-coupled receptor, GPR81. J Biol Chem. 2009;284(5):2811-22.

19. Langin D. Adipose tissue lipolysis revisited (again!): lactate involvement in insulin antilipolytic action. Cell Metab. 2010;11(4):242-3.

20. Moro C. Targeting cardiac natriuretic peptides in the therapy of diabetes and obesity. Expert Opin Ther Targets. 2016;20(12):1445-52. 
21. Wang TJ, Larson MG, Levy D, Benjamin EJ, Leip EP, Wilson PW, et al. Impact of obesity on plasma natriuretic peptide levels. Circulation. 2004;109(5):594-600.

22. Das SR, Drazner MH, Dries DL, Vega GL, Stanek HG, Abdullah SM, et al. Impact of body mass and body composition on circulating levels of natriuretic peptides: results from the Dallas Heart Study. Circulation. 2005;112(14):2163-8.

23. Khan AM, Cheng S, Magnusson M, Larson MG, Newton-Cheh C, McCabe EL, et al. Cardiac natriuretic peptides, obesity, and insulin resistance: evidence from two community-based studies. J Clin Endocrinol Metab. 2011;96(10):3242-9.

24. Magnusson M, Jujic A, Hedblad B, Engstrom G, Persson M, Struck J, et al. Low plasma level of atrial natriuretic peptide predicts development of diabetes: the prospective Malmo Diet and Cancer study. J Clin Endocrinol Metab. 2012;97(2):63845.

25. Gruden G, Landi A, Bruno G. Natriuretic peptides, heart, and adipose tissue: new findings and future developments for diabetes research. Diabetes Care. 2014;37(11):2899-908.

26. Sengenes C, Berlan M, De Glisezinski I, Lafontan M, Galitzky J. Natriuretic peptides: a new lipolytic pathway in human adipocytes. FASEB J. 2000;14(10):1345-51.

27. Sengenes $C$, Bouloumie $A$, Hauner $H$, Berlan $M$, Busse $R$, Lafontan $M$, et al. Involvement of a cGMP-dependent pathway in the natriuretic peptide-mediated hormone-sensitive lipase phosphorylation in human adipocytes. J Biol Chem. 2003;278(49):48617-26.

28. Moro C, Crampes F, Sengenes C, De Glisezinski I, Galitzky J, Thalamas C, et al. Atrial natriuretic peptide contributes to physiological control of lipid mobilization in humans. FASEB J. 2004;18(7):908-10.

29. Verboven K, Hansen D, Moro C, Eijnde BO, Hoebers N, Knol J, et al. Attenuated atrial natriuretic peptide-mediated lipolysis in subcutaneous adipocytes of obese type 2 diabetic men. Clin Sci (Lond). 2016;130(13):1105-14.

30. Ryden M, Backdahl J, Petrus P, Thorell A, Gao H, Coue M, et al. Impaired atrial natriuretic peptide-mediated lipolysis in obesity. Int J Obes (Lond). 2016;40(4):71420.

31. Stich V, de Glisezinski I, Crampes F, Suljkovicova H, Galitzky J, Riviere D, et al. Activation of antilipolytic alpha(2)-adrenergic receptors by epinephrine during exercise in human adipose tissue. Am J Physiol. 1999;277(4 Pt 2):R1076-83.

32. Stich V, de Glisezinski I, Galitzky J, Hejnova J, Crampes F, Riviere D, et al. Endurance training increases the beta-adrenergic lipolytic response in subcutaneous adipose tissue in obese subjects. Int J Obes Relat Metab Disord. 1999;23(4):374-81.

33. Polak J, Klimcakova E, Moro C, Viguerie N, Berlan M, Hejnova J, et al. Effect of aerobic training on plasma levels and subcutaneous abdominal adipose tissue gene expression of adiponectin, leptin, interleukin 6, and tumor necrosis factor alpha in obese women. Metabolism. 2006;55(10):1375-81.

34. Moro C, Pillard F, De Glisezinski I, Harant I, Rivi??Re D, Stich V, et al. Training Enhances ANP Lipid-Mobilizing Action in Adipose Tissue of Overweight Men. Medicine \& Science in Sports \& Exercise. 2005;37(7):1126-32.

35. Matthews DR, Hosker JP, Rudenski AS, Naylor BA, Treacher DF, Turner RC. Homeostasis model assessment: insulin resistance and beta-cell function from fasting plasma glucose and insulin concentrations in man. Diabetologia. 1985;28(7):412-9.

36. Blaak EE, Hul G, Verdich C, Stich V, Martinez A, Petersen M, et al. Fat oxidation before and after a high fat load in the obese insulin-resistant state. J Clin Endocrinol Metab. 2006;91(4):1462-9.

37. Wildman RP, Muntner P, Reynolds K, McGinn AP, Rajpathak S, Wylie-Rosett J, et al. The obese without cardiometabolic risk factor clustering and the normal weight with cardiometabolic risk factor clustering: prevalence and correlates of 2 phenotypes 
among the US population (NHANES 1999-2004). Arch Intern Med. 2008;168(15):1617-24.

38. Hickner RC, Rosdahl H, Borg I, Ungerstedt U, Jorfeldt L, Henriksson J. Ethanol may be used with the microdialysis technique to monitor blood flow changes in skeletal muscle: dialysate glucose concentration is blood-flow-dependent. Acta Physiol Scand. 1991;143(3):355-6.

39. Stich V, De Glisezinski I, Crampes F, Hejnova J, Cottet-Emard JM, Galitzky J, et al. Activation of alpha(2)-adrenergic receptors impairs exercise-induced lipolysis in SCAT of obese subjects. Am $J$ Physiol Regul Integr Comp Physiol. 2000;279(2):R499-504.

40. Polak J, Moro C, Bessiere D, Hejnova J, Marques MA, Bajzova M, et al. Acute exposure to long-chain fatty acids impairs \{alpha\}2-adrenergic receptor-mediated antilipolysis in human adipose tissue. J Lipid Res. 2007;48(10):2236-46.

41. Frayn KN. Calculation of substrate oxidation rates in vivo from gaseous exchange. J Appl Physiol Respir Environ Exerc Physiol. 1983;55(2):628-34.

42. Weir JB. New methods for calculating metabolic rate with special reference to protein metabolism. J Physiol. 1949;109(1-2):1-9.

43. Yumuk V, Tsigos C, Fried M, Schindler K, Busetto L, Micic D, et al. European Guidelines for Obesity Management in Adults. Obes Facts. 2015;8(6):402-24.

44. Bulow J. Human adipose tissue blood flow during prolonged exercise, III. Effect of beta-adrenergic blockade, nicotinic acid and glucose infusion. Scand J Clin Lab Invest. 1981;41(4):415-24.

45. Ardilouze JL, Karpe F, Currie JM, Frayn KN, Fielding BA. Subcutaneous adipose tissue blood flow varies between superior and inferior levels of the anterior abdominal wall. Int J Obes Relat Metab Disord. 2004;28(2):228-33.

46. Zouhal H, Lemoine-Morel S, Mathieu ME, Casazza GA, Jabbour G. Catecholamines and obesity: effects of exercise and training. Sports Med. 2013;43(7):591-600.

47. Lafontan M, Langin D. Lipolysis and lipid mobilization in human adipose tissue. Prog Lipid Res. 2009;48(5):275-97.

48. Jocken JW, Goossens GH, Boon H, Mason RR, Essers Y, Havekes B, et al. Insulinmediated suppression of lipolysis in adipose tissue and skeletal muscle of obese type 2 diabetic men and men with normal glucose tolerance. Diabetologia. 2013;56(10):2255-65.

49. Hershkop K, Besor O, Santoro N, Pierpont B, Caprio S, Weiss R. Adipose Insulin Resistance in Obese Adolescents Across the Spectrum of Glucose Tolerance. J Clin Endocrinol Metab. 2016;101(6):2423-31.

50. Zhang J, Hupfeld CJ, Taylor SS, Olefsky JM, Tsien RY. Insulin disrupts betaadrenergic signalling to protein kinase A in adipocytes. Nature. 2005;437(7058):56973.

51. Ahmed K, Tunaru S, Tang C, Muller M, Gille A, Sassmann A, et al. An autocrine lactate loop mediates insulin-dependent inhibition of lipolysis through GPR81. Cell Metab. 2010;11(4):311-9.

52. Follenius $M$, Brandenberger $\mathrm{G}$. Increase in atrial natriuretic peptide in response to physical exercise. Eur J Appl Physiol Occup Physiol. 1988;57(2):159-62.

53. Lonnroth $\mathrm{P}$, Smith $U$. The antilipolytic effect of insulin in human adipocytes requires activation of the phosphodiesterase. Biochem Biophys Res Commun. 1986;141(3):1157-61.

54. Makino $H$, Suzuki T, Kajinuma $H$, Yamazaki M, Ito $H$, Yoshida S. The role of insulinsensitive phosphodiesterase in insulin action. Adv Second Messenger Phosphoprotein Res. 1992;25:185-99.

55. Kovacova Z, Tharp WG, Liu D, Wei W, Xie H, Collins S, et al. Adipose tissue natriuretic peptide receptor expression is related to insulin sensitivity in obesity and diabetes. Obesity (Silver Spring). 2016;24(4):820-8. 
56. Polak J, Moro C, Klimcakova E, Hejnova J, Majercik M, Viguerie N, et al. Dynamic strength training improves insulin sensitivity and functional balance between adrenergic alpha $2 \mathrm{~A}$ and beta pathways in subcutaneous adipose tissue of obese subjects. Diabetologia. 2005;48(12):2631-40.

57. De Glisezinski I, Crampes F, Harant I, Berlan M, Hejnova J, Langin D, et al. Endurance training changes in lipolytic responsiveness of obese adipose tissue. Am J Physiol. 1998;275(6 Pt 1):E951-6.

58. Richterova B, Stich V, Moro C, Polak J, Klimcakova E, Majercik M, et al. Effect of endurance training on adrenergic control of lipolysis in adipose tissue of obese women. J Clin Endocrinol Metab. 2004;89(3):1325-31.

59. Moro C, Pasarica M, Elkind-Hirsch K, Redman LM. Aerobic exercise training improves atrial natriuretic peptide and catecholamine-mediated lipolysis in obese women with polycystic ovary syndrome. J Clin Endocrinol Metab. 2009;94(7):257986. 


\section{SUPPLEMENTARY MATERIAL}

A

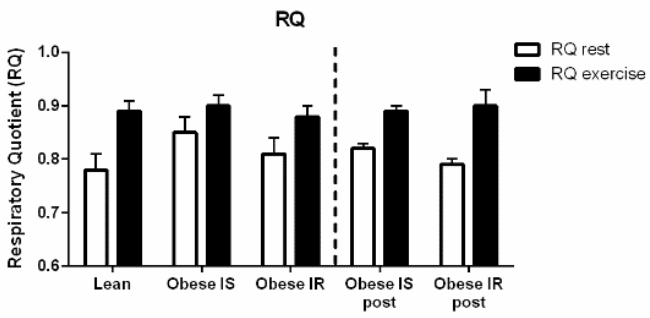

B

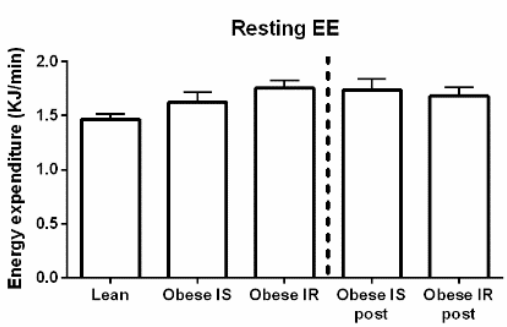

C

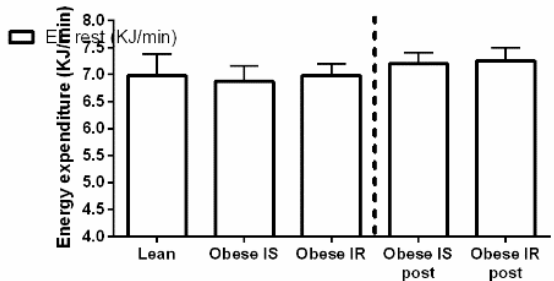

D

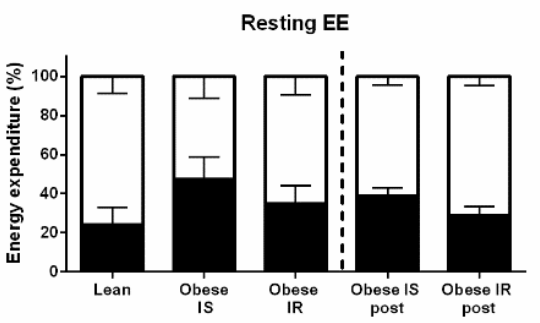

E

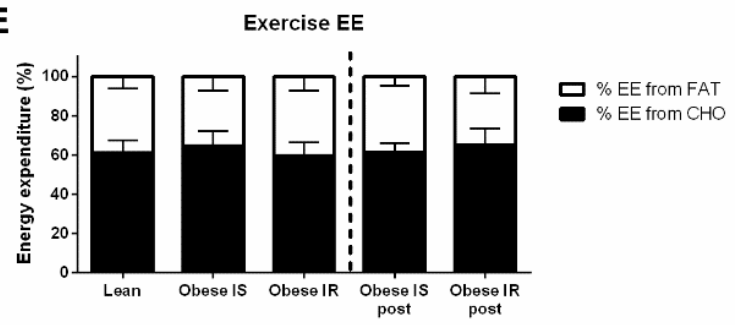

SUPPLEMENTARY FIGURE S1. Fasting and exercise-induced whole-body energy expenditure and substrate oxidation before and after exercise intervention.

Indirect calorimetry was performed during resting conditions and during an acute exercise bout of moderate intensity. Mean O2-consumption and CO2-production over 10 min were used for calculations of respiratory quotient $(A)$, energy expenditure and substrate oxidation during fasting $(B+D)$ and during exercise $(C+E)$. Post intervention data are added for both obese groups $(A-E)$. CHO, carbohydrates; $E E$, energy expenditure; IR, insulin resistant; IS, insulin sensitive. Data are presented as mean \pm SEM. 
Chapter 7 


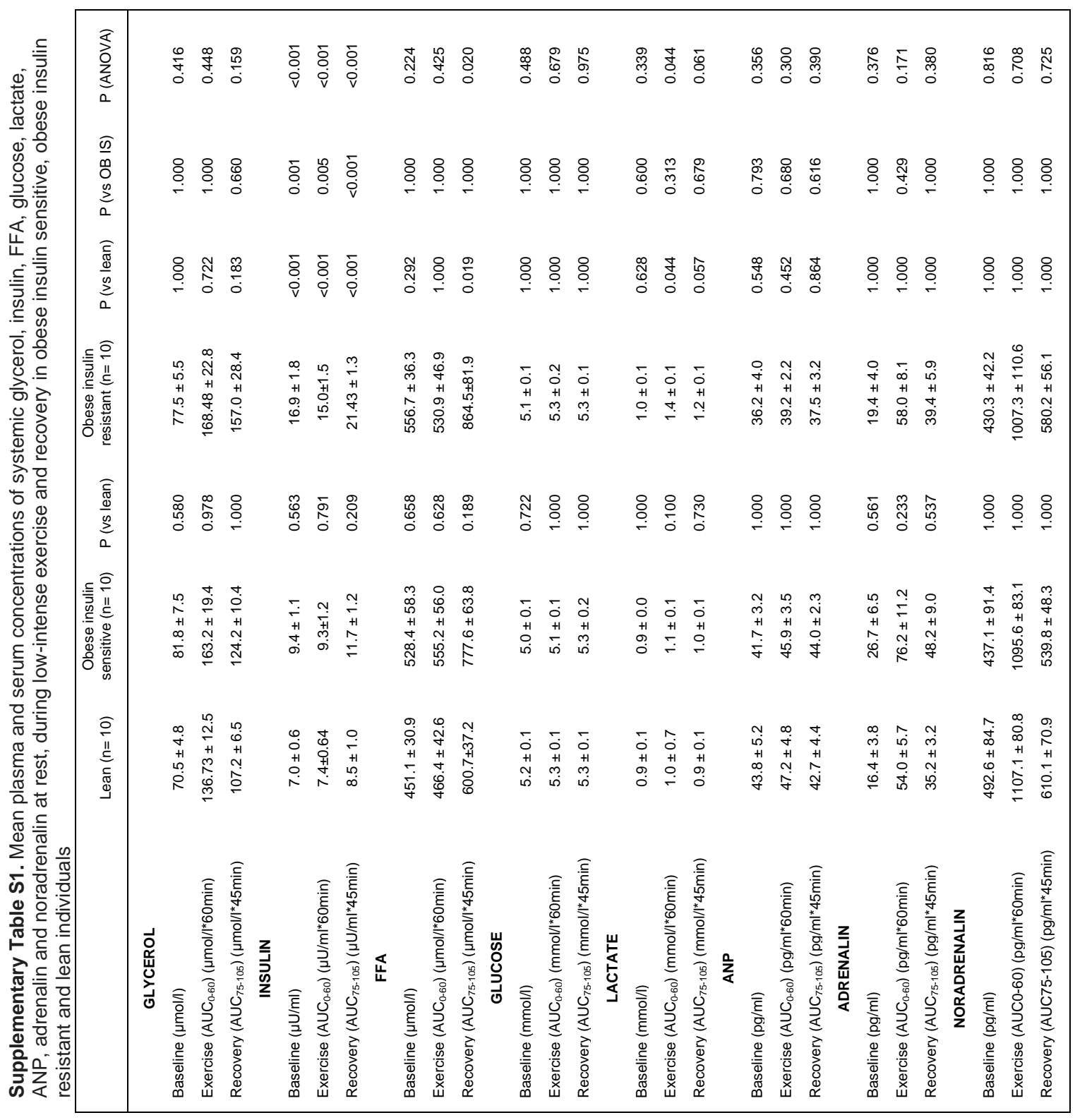

Data are mean \pm SEM. $A \cup C_{0-60}$ (area under the curve during exercise (from timepoint 0 till $60 \mathrm{~min}$ )), $\mathrm{AUC}_{75-105}$ (area under the curve during the recovery period (from timepoint 75 until 105min)). Group effects were tested with a one-way ANOVA with Bonferroni post-hoc. 


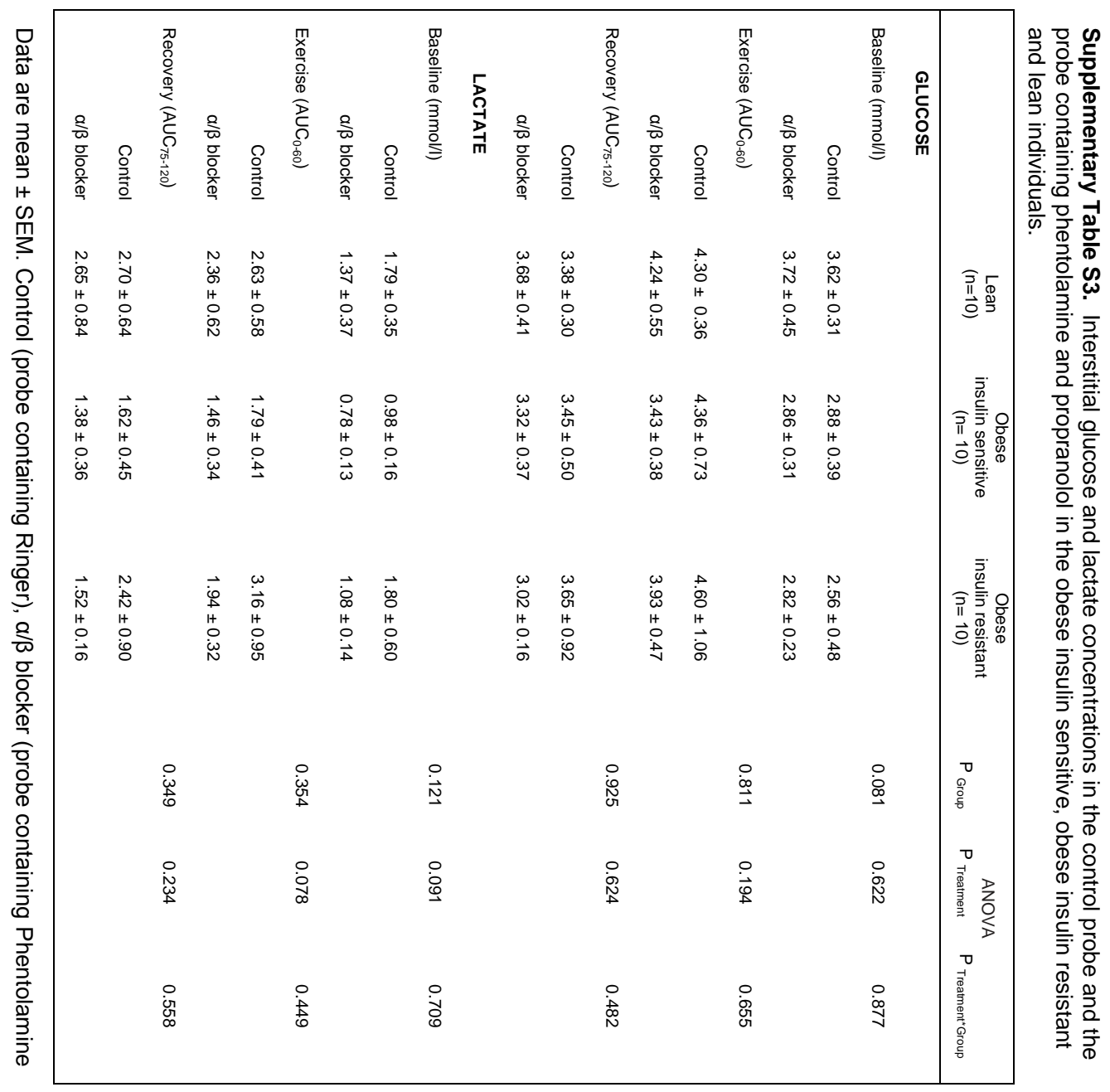




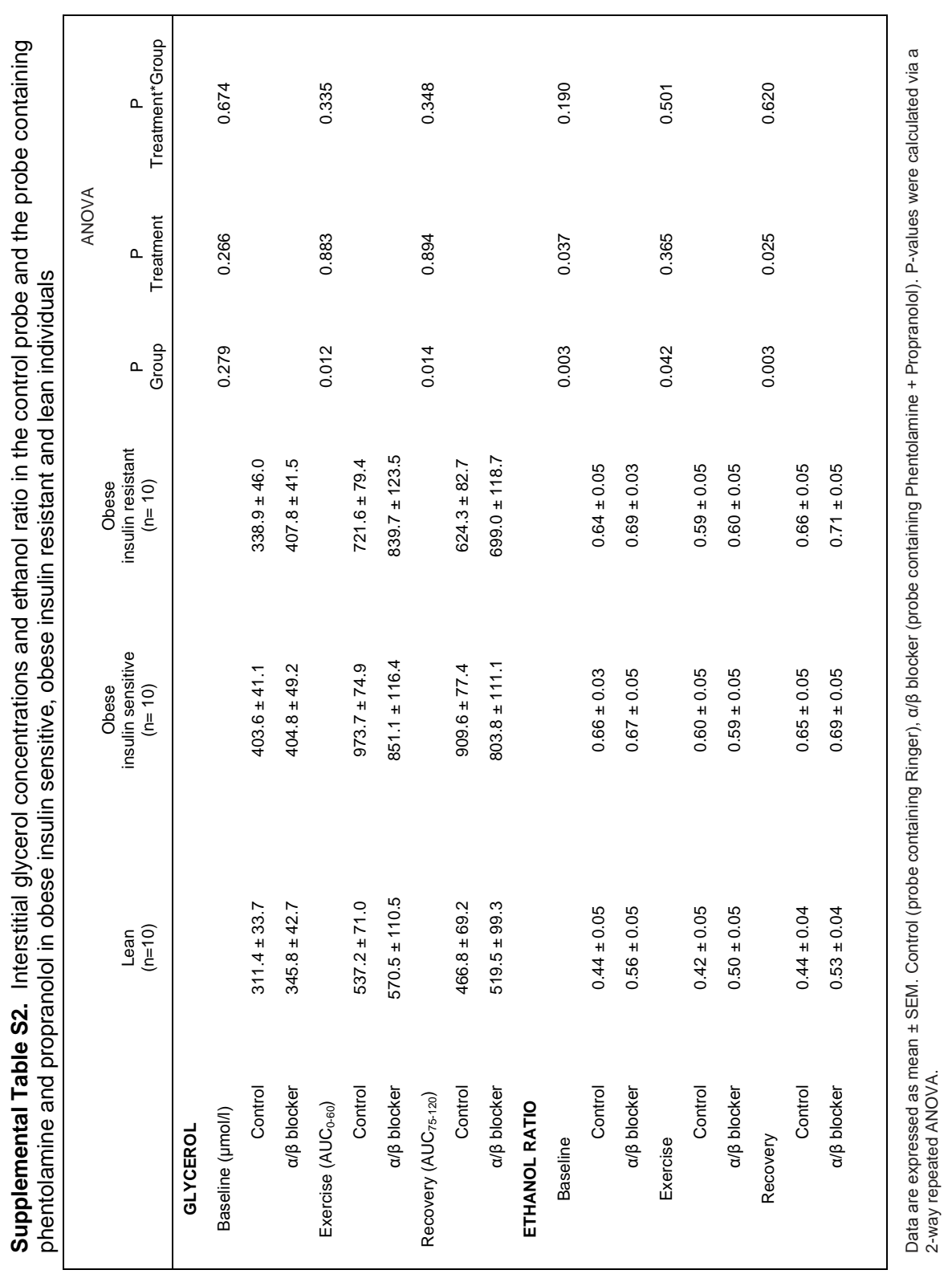


Chapter 7 


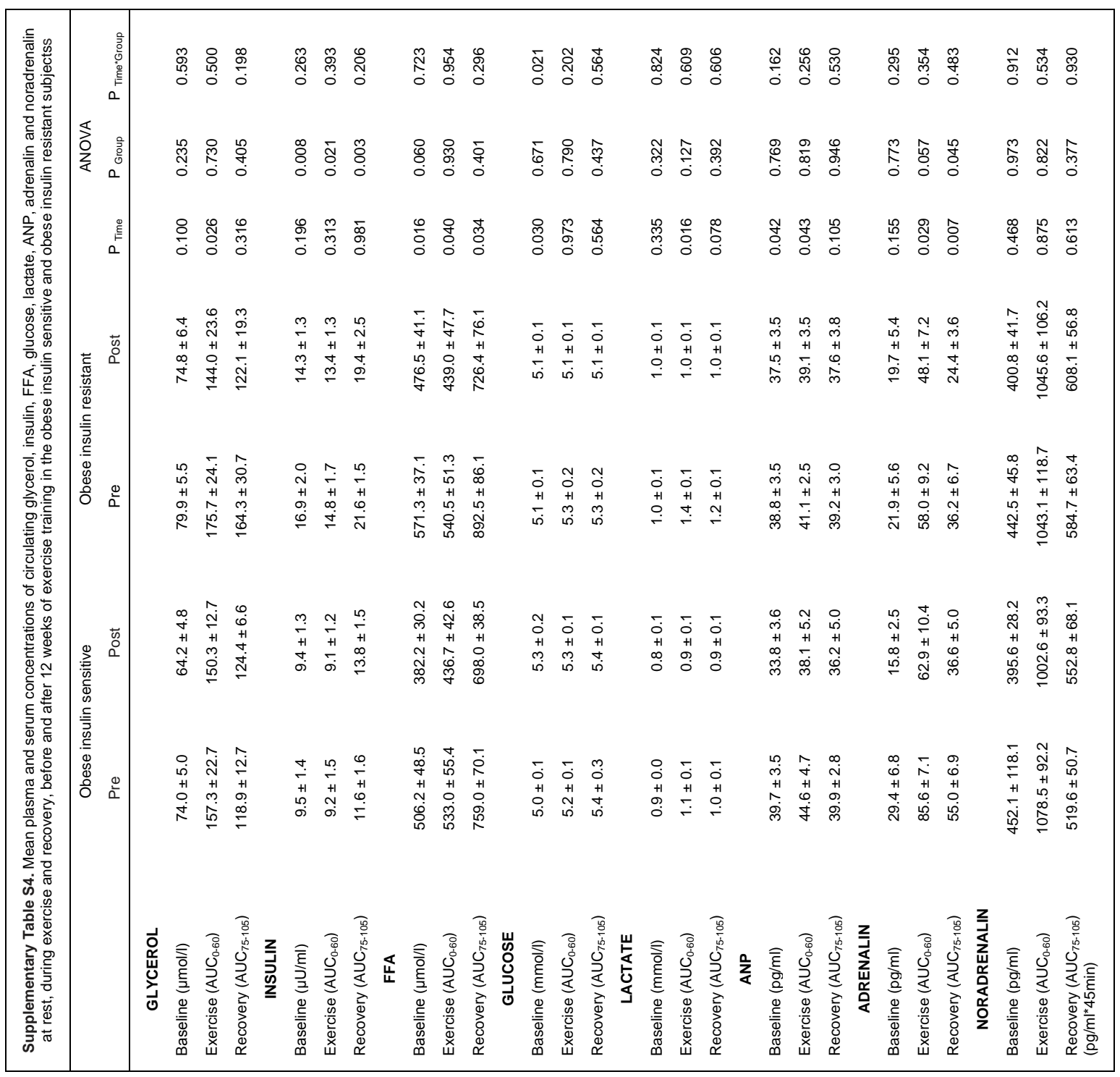

Data are mean \pm SEM. AUC0-60 (area under the curve during exercise (from timepoint 0 till $60 \mathrm{~min}$ )), AUC75-105 (area under the curve during the recovery period (from timepoint 75 until 105min)). Group effects were tested with a one-way ANOVA with Bonferroni post-hoc. 
CHAPTER 8 
GENERAL DISCUSSION 
The prevalence of obesity has increased enormously over the last decades and is associated with an increased risk for metabolic impairments and chronic diseases such as insulin resistance [1], type 2 diabetes [2] and cardiovascular diseases [3] Obesity results from a chronic positive energy balance that leads to an increased amount of adipose tissue mass. However, adipose tissue mass per se does not seem to be the most important contributor to the development of obesity-related disorders. Body fat distribution and adipose tissue dysfunction play a more prominent role in the determination of cardiometabolic health $[4,5]$. Therapies to reduce obesity and related comorbidities can include dietary manipulation (as extensively discussed in Chapter 2), physical activity strategies and a pharmacological approach.

There is evidence to suggest that both the renin-angiotensin system (RAS) and the natriuretic peptide (NP) system can influence cardiometabolic risk. Indeed, several RAS components, which are also present in different key metabolic organs such as the adipose tissue, skeletal muscle and the liver, are increased in obesity and insulin resistance [6-9]. Also, reduced circulating NP concentrations have been observed in obesity and type 2 diabetes [10]. An increased RAS activity [6] and reduced NP concentrations [10] have detrimental metabolic effects and may increase disease progression [11-15]. Recently, a novel dual acting drug, sacubitril/valsartan, has been developed that facilitates the beneficial effects of the NP system, while inhibiting the detrimental effects of the RAS [16]. This combination therapy may have beneficial synergistic effects $[17,18]$ with respect to disease progression. Indeed, sacubitril/valsartan has been shown to be superior in reducing the risks of cardiovascular death or hospitalization for heart failure as compared to monotherapy with enalapril [19]. Due to the common pathophysiological impairments in individuals with cardiovascular and several metabolic diseases (e.g. reduced oxidative capacity, altered lipolysis, increased inflammation, insulin resistance), treatment with sacubitril/valsartan could induce clinical benefits in both patients with cardiovascular and/or metabolic diseases. However, it remains to be established whether sacubitril/valsartan may also have (superior) beneficial metabolic effects as compared to other blood pressure lowering agents.

Beside a pharmacological approach, changes in lifestyle are effective in preventing the development of type 2 diabetes and related cardiometabolic complications [20, 21]. Increasing physical activity levels lead to increased energy expenditure, improved physical fitness and contribute to an improved metabolic health [22, 23], which could be attributable to changes in adipose tissue metabolism. Although several rodent studies suggest that exercise training may improve adipose tissue metabolism and function [24], human data are limited and need further investigation [24-26].

Therefore, in the present thesis, the metabolic effects of pharmacological treatment with sacubitril/valsartan, as well as exercise-training induced effects on adipose tissue metabolism and the metabolic profile were investigated. In this chapter, our findings will be discussed and put into a broader perspective. 


\section{PHARMACOLOGICAL TREATMENT WITH SACUBITRIL/VALSARTAN}

Combination therapies that simultaneously target more than one biological pathway or mechanism may be more effective in reducing disease progression because of additional and/or synergistic effects as compared to monotherapies [17, 18]. Therefore, due to common involved pathways, it is tempting to postulate that combination therapy using sacubitril/valsartan through its distinct mechanisms of action, may target risk factors for both cardiovascular (e.g. hypertension) and metabolic diseases such as an impaired lipid mobilization, lipid oxidation and substrate utilization, as will be discussed below.

\section{Effects on insulin sensitivity}

In Chapter 3, we investigated the effects of 8 weeks treatment with sacubitril/valsartan in obese hypertensive patients on peripheral insulin sensitivity. Compared to the metabolically neutral comparator, the calcium antagonist amlodipine, peripheral insulin sensitivity was significantly improved after sacubitril/valsartan, independent of changes in body weight or waist circumference. The increased peripheral insulin sensitivity may be due to valsartan-induced $\mathrm{AT}_{1}$ receptor inhibition, increased availability of neprilysin (NEP) substrates or both mechanisms combined. Unfortunately, the present study design does not allow any conclusions regarding the separate effects of valsartan and sacubitril on peripheral insulin sensitivity.

The improvement in peripheral insulin sensitivity following sacubitril/valsartan is in line with most [27-31] but not all [32] previously performed human studies using valsartan treatment. Importantly, however, the latter study [32] investigated the effects of a lower dosage of valsartan ( $40 \mathrm{mg} /$ day vs. $400 \mathrm{mg} /$ day in our study) after 4 weeks of treatment and used surrogate markers of insulin sensitivity rather than the hyperinsulinemic-euglycemic clamp, which is the golden-standard. Taken together, our data and previous studies collectively show that valsartan improves peripheral insulin sensitivity in humans.

Although it has been shown that interference with the RAS improves glucose metabolism and reduces the incidence of type 2 diabetes [6], the valsartan-induced effects may not solely explain the observed improvement in peripheral insulin sensitivity with sacubitril/valsartan treatment in the present study and most likely also NEP inhibition is involved. The effects of NEP inhibition per se on insulin sensitivity have not been investigated previously, although it is known that a reduced NP system activity is associated with insulin resistance and type 2 diabetes and that modulation of the NP system can be protective against the development of insulin resistance [10].

An increased adipose tissue lipolysis, accompanied by an increased fatty acid oxidation has beneficial effects on insulin sensitivity [33, 34]. Therefore, we hypothesized that enhanced lipolysis and whole-body fat oxidation contributed to the improved peripheral insulin sensitivity following sacubitril/valsartan treatment, as described in Chapter $\mathbf{3}$ and Chapter $\mathbf{4}$, since it has been shown that activation of NP signaling induces lipolysis [35-39], promotes fatty acid oxidation [36, 37, 40] and enhances mitochondrial oxidative metabolism [41-43].

While monotherapy with valsartan increased peripheral insulin sensitivity by approximately $10 \%$ in subjects with impaired glucose metabolism [27, 44], 
treatment with sacubitril/valsartan showed an increase of $\sim 20 \%$ in insulin sensitivity, with no marked effects of amlodipine treatment. Although the previous study [27] used a lower dosage of valsartan as compared to our study (320 mg/day vs. $400 \mathrm{mg}$ per day, respectively), this difference is not likely to explain the additional $10 \%$ increase in insulin sensitivity after treatment with sacubitril/valsartan. Therefore, these data suggest that the NEP inhibitor sacubitril has significantly contributed to the effects on peripheral insulin sensitivity that were found in the present study.

\section{Effects on subcutaneous adipose tissue lipolysis and substrate oxidation}

Since adipose tissue metabolism and function and substrate oxidation are important factors that contribute to peripheral insulin sensitivity, we investigated sacubitril/valsartan-mediated effects on abdominal subcutaneous adipose tissue lipolysis, whole-body lipolysis and substrate oxidation, both at rest and during an acute bout of exercise.

After 8 weeks of treatment, there was a slight, but significant increase in abdominal subcutaneous adipose tissue lipolysis at rest in the sacubitril/valsartan as compared to the amlodipine group (Chapter 3). Surprisingly, the increased subcutaneous adipose tissue lipolysis at rest did not translate into significant changes in whole-body lipolysis, as measured with the stable isotope [1,1,2,3,3$\left.{ }^{2} \mathrm{H}\right]$-glycerol (Chapter 3). Together, these findings either suggest that the increased subcutaneous adipose tissue lipolysis might be paralleled by a reduced lipolysis in other adipose tissue depots or, more likely, that the effects on lipolysis may not be physiologically relevant. An increased adipose tissue lipolysis has beneficial effects on insulin sensitivity, only when the mobilized fatty acids are oxidized at an increased rate [33, 34].

In Chapter 3, we found no significant changes in total energy expenditure and substrate oxidation in resting conditions. In Chapter 4, we examined the effects of sacubitril/valsartan on abdominal subcutaneous and whole-body lipolysis during an acute bout of aerobic exercise, which is known to stimulate lipolysis [45, 46], and observed no significant differences in abdominal subcutaneous adipose tissue lipolysis, whole-body lipolysis, energy expenditure and substrate oxidation between groups. Thus, there were no differences between resting (Chapter 3) and exercise (Chapter 4) conditions, except for a slightly higher abdominal subcutaneous adipose tissue lipolysis under resting conditions (Chapter 3 ). Therefore, an increased fat oxidation does not seem to contribute to the improved peripheral insulin sensitivity following sacubitril/valsartan treatment.

Next, we examined the effects of sacubitril/valsartan on adipose tissue gene and protein expression, as described in Chapter 5, and we found no significant changes in the expression of genes and proteins of factors involved in lipolysis, natriuretic peptide signaling and mitochondrial oxidative metabolism.

These results are in line with a previous study that did not show an increased adipose tissue lipolytic gene and protein expression after 26 weeks of valsartan treatment [44]. Furthermore, $\mathrm{AT}_{1}$-receptor blockade did not increase abdominal subcutaneous adipose tissue lipolysis [47] and, therefore, it seems unlikely that $\mathrm{AT}_{1}$-receptor blockade with sacubitril/valsartan treatment contributed to the mild alteration in lipid mobilization. Rather, a NEP inhibition-mediated increased NP 
availability could have contributed to the observed effects on subcutaneous adipose tissue lipolysis, since NP have been shown to affect adipose tissue lipid mobilization and oxidation in lean and overweight men and women [35-40].

Taken together, alterations in adipose tissue lipolysis or whole-body substrate oxidation at rest and during exercise do not seem to contribute to the sacubitril/valsartan-induced improvement in peripheral insulin sensitivity. The underlying mechanisms remain to be elucidated, but seem to be explained by metabolic effects in other tissues (e.g. visceral adipose tissue, the liver or skeletal muscle).

\section{EXERCISE TRAINING INTERVENTIONS TO IMPROVE METABOLIC HEALTH}

Regular physical exercise has beneficial effects on cardiometabolic health and improves glucose tolerance, insulin sensitivity and circulating lipid concentrations $[48,49]$. These exercise-induced improvements in cardiometabolic risk profile have largely been attributed to changes in skeletal muscle metabolism and function, but physical exercise may also induce alterations in other metabolically active tissues, including the adipose tissue. Indeed, several rodent studies suggest that exercise training may improve adipose tissue metabolism and function [24], although the evidence for this in humans is limited [24-26].

\section{Effects on insulin sensitivity}

In Chapter $\mathbf{6}$ and Chapter $\mathbf{7}$ of this thesis, we investigated the metabolic effects of a 12-week supervised, combined endurance and resistance exercise training program in sedentary, middle-aged overweight/obese men with or without metabolic impairments (Chapter 6) and sedentary, middle-aged obese insulin sensitive, obese insulin resistant and age-matched lean insulin sensitive men (Chapter 7).

Both exercise training programs improved aerobic capacity, maximal power output and maximal muscle strength, indicating that the supervised, progressive nature of both programs was successful with respect to enhancement of physical fitness. This enhanced physical fitness was accompanied by improved peripheral insulin sensitivity, as determined using either a hyperinsulinemic-euglycemic clamp (Chapter 6) or HOMA-IR (Chapter 7). These findings are in line with previous studies showing an improved insulin sensitivity after 12 weeks of exercise in obese individuals $[50,51]$ and could, among other mechanisms, be explained by an altered body composition [52]. In Chapter 6 , we observed a modest but significant reduction in body fat mass $(\sim 0.7 \mathrm{~kg})$ and body fat percentage $(\sim 0.6 \%)$ in both obese groups, without significant changes in fat-free mass. In Chapter 7, we observed a significantly reduced fat mass $(\sim 2.1 \mathrm{~kg}$ in the obese insulin sensitive group and $\sim 1.9 \mathrm{~kg}$ in the obese insulin resistant group), body fat percentage ( 1.6 $\%$ in the obese insulin sensitive and $\sim 0.9 \%$ in the obese insulin resistant group), $\mathrm{BMI}$ and body weight, while fat-free mass remained unchanged and was not different between groups. Previous data showed that increased fat-free mass (e.g. skeletal muscle cross-sectional area) and improved skeletal muscle function (e.g. skeletal muscle capillarization, oxidative capacity) are associated with improved insulin sensitivity [53-56], while reduced fat-free mass induced insulin resistance 
$[57,58]$. However, our data suggest that peripheral insulin sensitivity can improve without significant changes in fat-free mass.

Although exercise training significantly increased peripheral insulin sensitivity it did not induce changes in adipose tissue and hepatic insulin sensitivity, as described in Chapter 6. These findings are in contrast with previous rodent [59,60] and human [61] studies. The unaltered adipose tissue and hepatic insulin sensitivity may be explained by the rather minor changes in fat mass loss. Nevertheless, our data showed that exercise training beneficially improved physical fitness, body composition and peripheral insulin sensitivity, irrespective of the baseline metabolic status.

\section{Effects on subcutaneous adipose tissue metabolism and adipokine expression}

Subcutaneous adipocyte size is closely associated with adipose tissue function and insulin resistance [4] and adipocyte hypertrophy is correlated with impairments in adipose tissue metabolism [62]. Since marked weight loss is associated with decreased adipocyte size in subcutaneous adipose tissue, which is accompanied by improved insulin sensitivity [63-66], we investigated if exercise training could alter adipocyte morphology, thereby contributing to an improved subcutaneous adipose tissue metabolism and whole-body insulin sensitivity.

In Chapter 6, we found that physical exercise training did not change abdominal subcutaneous adipocyte morphology, neither mean adipocyte size nor the proportion of small and large adipocytes, in metabolically healthy obese and metabolically compromised obese subjects. In contrast, a previous study demonstrated a decreased adipocyte size in young men, but not in women, after 20 weeks of endurance training [67]. Importantly, in the latter study, a more pronounced reduction in body weight $(\sim 3.0 \mathrm{~kg})$ was achieved. Since we observed a weight reduction of only $\sim 0.7 \mathrm{~kg}$ in Chapter 6 , it is likely that a more pronounced decrease in adipose tissue mass is needed to induce significant changes in adipocyte morphology and, consequently, adipose tissue metabolism. While exercise training duration was comparable between the exercise training studies in chapter 6 and 7, we observed a greater reduction in fat mass in Chapter 7, which is likely due to the higher training volume. In Chapter 6, subjects performed three training sessions per week, of which two sessions consisted of $30 \mathrm{~min}$ cycling at $70 \% \mathrm{VO}_{2 \max }$ and one training session included resistance training at $60 \%$ of $1 \mathrm{RM}$ (3 sets of 10 reps). In Chapter 7, the participants performed three training sessions per week of which all consisted of $45 \mathrm{~min}$ cycling at $65 \% \mathrm{VO}_{2 \max }$ combined with resistance training at $65 \%$ of $1 \mathrm{RM}$ (4 sets of 10 reps). Since a greater reduction in body weight (fat mass) induces more favorable metabolic effects $[68,69]$, the greater reduction in fat mass, as observed in Chapter 7, could have altered adipocyte morphology and improved adipose tissue metabolism, although this was not investigated.

However, it remains to be established how much weight loss is needed to induce a significant reduction in adipocyte size and which exercise training modality is most optimal $[63,68,70]$. 
Since adipocyte hypertrophy is associated with impairments in adipose tissue metabolism [4, 62], the minor reduction in fat mass and the lack of changes in adipocyte morphology seems to explain the unaltered expression of genes and proteins related to adipose tissue metabolism, as reported in Chapter 6. More specific, there were no exercise training-induced changes in expression of genes involved in inflammation (e.g. TNF-a, IL-6, MCP-1 and CD68), which have been implicated to induce insulin resistance. Additionally, gene expression of the adipokines leptin and adiponectin, which are related to adipocyte size [71, 72], also remained unchanged after exercise training. These observations are in line with most other exercise training studies in obese subjects [73-78]. Furthermore, an adequate mitochondrial function is essential to maintain adipose tissue function, glucose homeostasis [79] and protects against insulin resistance and type 2 diabetes [80]. Browning of white adipose tissue may increase cellular energy expenditure [81, 82], thereby improving whole-body glucose homeostasis and insulin sensitivity in humans [83]. We did not found changes in browning markers after training, which is in line with studies in lean $[84,85]$ and overweight $[85,86]$ subjects, but in contrast to rodent data [41, 87]. Furthermore, the exercise training intervention did not induce changes in mitochondrial gene expression and did not alter mitochondrial oxidative phosphorylation protein expression, which was in accordance with most [78] [84, 86, 88], but not all previous studies [89, 90]. When investigating adipose tissue lipolysis, which is one of the characteristics of adipose tissue dysfunction and relates to peripheral insulin resistance [91], we observed no major significant exercise training-induced effects on expression of genes related to lipolysis. Furthermore, ex vivo basal and maximal lipolysis, as well as $\beta_{2^{-}}$ adrenergic sensitivity of lipolysis in mature human adipocytes, were not affected by the exercise training program. The latter observation is in agreement with a previous study in obese non-diabetic men [92] and may be explained by the minor change in fat mass loss, since it has previously been demonstrated that substantial weight loss (BMI: $-6.1 \mathrm{~kg} / \mathrm{m}^{2}$ ) increased and normalized the sensitivity to catecholamine-stimulated lipolysis in obese subjects [93].

Overall, Chapter 6 demonstrated that a 12-weeks supervised, progressive exercise training intervention did not significantly affect abdominal subcutaneous adipocyte morphology, adipose tissue gene and protein expression of markers related to adipose tissue function, nor $\beta_{2}$-adrenergic sensitivity in obese subjects, irrespective of their baseline metabolic status.

Disturbances in subcutaneous adipose tissue (SCAT) lipolysis have been reported in obese humans [46], including a reduced adrenergically-mediated lipolysis [9496]. On the other hand, non-adrenergically-mediated lipolysis in SCAT substantially contributes to lipolysis during exercise in healthy young lean [38] and healthy young overweight men [39]. Atrial natriuretic peptide (ANP) is the most potent stimulator of human adipose tissue lipolysis [35] and one of the major lipolytic hormones produced upon exercise [35, 97]. However, ANP responsiveness has been shown to be impaired in middle-aged obese men with or without type 2 diabetes [98] and in middle-aged obese women and overweight men [99].

It has been shown that endurance exercise training can partly improve $\beta$ adrenoceptor sensitivity and reduce anti-lipolytic $\alpha_{2}$-adrenoceptor sensitivity in human SCAT [76, 100, 101], and can alleviate ANP-mediated lipolysis in 
subcutaneous adipocytes in young, metabolically healthy overweight individuals [102, 103]. However, it remained to be investigated if combined endurance and resistance exercise training could improve ANP-mediated lipolysis in metabolically compromised conditions.

In Chapter 7, we aimed to investigate the effects of local combined $\alpha$ - and $\beta$ adrenergic blockade on SCAT lipolysis in obese insulin sensitive, obese insulin resistant and age-matched lean insulin sensitive men. Therefore, we determined in situ SCAT lipolysis at rest, during a single bout of low-intensity endurance exercise and during recovery in age-matched obese insulin sensitive, obese insulin resistant and lean insulin sensitive men in the presence or absence of combined $\alpha$ - and $\beta$ adrenergic receptor blockade in abdominal SCAT. Next, the obese individuals participated in a 12-week combined endurance and resistance exercise training intervention to investigate whether exercise training was able to improve (non-) adrenergic-mediated abdominal SCAT lipolysis in obese insulin resistant subjects.

Our findings indicated a major contribution of non-adrenergically mediated lipolysis during exercise in abdominal SCAT of lean and obese individuals. Additionally, we found a greater increase in extracellular glycerol concentration in SCAT during exercise in obese insulin sensitive as compared to lean insulin sensitive individuals. Although extracellular glycerol concentration is a reflection of lipolysis, the higher exercise-induced lipolysis in the obese insulin sensitive group is likely explained by the lower adipose tissue blood flow (ATBF) in the obese state, which contributes to higher extracellular glycerol concentrations due to a lower removal of glycerol from the adipose tissue [39, 104]. Local $\alpha$-/ $\beta$-adrenergic blockade substantially reduced basal ATBF in lean but not in the obese individuals, which suggests that adrenergic sensitivity of ATBF is reduced in the obese state, which has been shown before $[105,106]$.

The exercise-induced SCAT lipolysis following combined $\alpha$-/ $\beta$-adrenergic blockade was substantially reduced in obese insulin sensitive but not in lean insulin sensitive or obese insulin resistant men. This might suggest differences in adrenergic receptor expression and/or sensitivity. Previous data showed that the lipolytic activity of the $\beta$-adrenergic receptors is attenuated in obese insulin resistant individuals [95, 96, 107], while inhibitory $\alpha_{2}$-adrenoceptors become predominant on adipocytes in the obese insulin resistant state [107, 108] and reduce exercisemediated SCAT lipolysis [105]. Additionally, obese insulin resistant individuals often show lower plasma catecholamine (adrenaline, noradrenaline) responses to physical exercise [109], although we did not find this in Chapter 7. Furthermore, the blunted SCAT lipolysis in the obese insulin resistant individuals, as compared to the obese insulin sensitive group, might also partly be explained by the significantly higher insulin concentrations, as shown previously [110]. In addition, increased plasma lactate concentrations, as observed in the obese insulin resistant individuals, may have contributed to the reduced lipolytic response, since lactate has been shown to inhibit lipolysis in mice [111] and human primary adipocytes in vitro [112].

In all groups, we found a major contribution of non-adrenergic components in SCAT lipolysis during low-intensity exercise, which is likely involving ANP-mediated lipolysis. This observation is in line with previous studies, which showed that nonadrenergically-mediated mechanisms are involved in SCAT lipolysis, accounting for 
$\sim 65 \%$ of the exercise-mediated lipolysis in young healthy lean and overweight men $[38,39]$. We found that SCAT lipolysis in obese insulin resistant men, compared to the obese insulin sensitive group, was not affected by local $\alpha$-/ $\beta$-adrenergic blockade, which may point toward an interaction between SCAT adrenergicallymediated lipolysis and whole-body insulin resistance. These findings support a catecholamine-resistant phenotype of the SCAT during exercise, especially in the obese insulin resistant state.

The 12-week combined endurance and resistance exercise training intervention did not improve exercise-induced abdominal SCAT lipolysis. Furthermore, the efficiency of local $\alpha$ - $/ \beta$-adrenergic blockade was not affected and these data combined suggest that the lipolytic disturbances in SCAT of obese insulin resistant individuals remain unaffected after 12 weeks of exercise training. This observation is in contrast with previous studies that showed beneficial changes in SCAT insulin sensitivity [61], SCAT adrenergic sensitivity [61, 92, 113] as well as ANP-mediated lipolysis [114] after exercise training in obese subjects.

Taken together, the results described in Chapter 7 show a major role for nonadrenergically-mediated lipolysis in SCAT during low-intensity exercise, likely involving ANP-mediated lipolysis. Furthermore, our data suggest that even after a substantial improvement in body composition, physical fitness and insulin sensitivity, lipolytic disturbances remain unaffected in SCAT of obese insulin resistant individuals after a 12-week exercise training intervention.

\section{CONCLUSION AND FUTURE PERSPECTIVES}

This thesis describes the effects of a pharmacological intervention as well as physical exercise interventions to improve metabolic health in obese individuals, with a focus on adipose tissue metabolism.

In Chapter 3, we found improved peripheral insulin sensitivity after 8 weeks of combination therapy with sacubitril/valsartan in obese hypertensive subjects, which was accompanied by an increased abdominal subcutaneous adipose tissue lipolysis at rest. However, this finding did not translate into alterations in wholebody lipolysis, energy expenditure and substrate oxidation. In Chapter 4, no changes were found for these parameters during a single bout of moderateintensity endurance exercise. Therefore, it seems that sacubitril/valsartan had no clinically relevant effects on adipose tissue and whole-body lipolysis and substrate utilization. In line, in Chapter 5, no significant effects on gene and protein expression of markers related to lipolysis, natriuretic peptide signaling and mitochondrial oxidative metabolism were found. Therefore, we conclude that treatment with sacubitril/valsartan improved peripheral insulin sensitivity, without altering the abdominal subcutaneous adipose tissue metabolic phenotype and substrate oxidation.

In Chapter 6, we showed that a 12-weeks supervised, progressive, combined endurance and resistance exercise training program improved body composition, physical fitness and peripheral insulin sensitivity in sedentary, middle-aged overweight/obese men with or without metabolic impairments. However, no significant effects on hepatic and adipose tissue insulin sensitivity, abdominal 
subcutaneous adipocyte morphology, adipose tissue gene and protein expression of markers related to adipose tissue function, nor $\beta_{2}$-adrenergic sensitivity of abdominal SCAT lipolysis were observed in obese subjects, irrespective of their baseline metabolic status.

In Chapter 7, we showed a major contribution of non-adrenergically-mediated lipolysis during exercise in SCAT of sedentary, middle-aged obese insulin sensitive, obese insulin resistant and age-matched lean insulin sensitive men. Furthermore, 12 weeks of supervised, combined endurance and resistance exercise training did not significantly improve exercise-induced abdominal SCAT lipolysis.

The outcomes of this thesis provide evidence for an improved metabolic health after sacubitril/valsartan treatment and exercise training interventions, but several questions and issues should be addressed in future research:

1. Since we used a combination drug, we cannot distinguish between the individual effects of sacubitril (NEP inhibition) and valsartan (RAS blockade) on the improved peripheral insulin sensitivity. It would be of interest to investigate the effects of NEP inhibition compared to placebo, valsartan (at a similar dosage as applied in this thesis), or a metabolically neutral blood pressure lowering agent and/or sacubitril/valsartan, to elucidate whether the observed effects of sacubitril/valsartan on peripheral insulin sensitivity are attributable to the slightly higher valsartan concentration as compared to a previous study [27], or whether the effects are attributable to the additional effects of increased neprilysin substrates, such as NP concentrations.

2. We treated obese hypertensive patients with sacubitril/valsartan for 8 weeks, since literature reports show that 8 weeks of treatment is sufficiently long to demonstrate metabolic effects of several drug classes used for the treatment of hypertension and heart failure, including ACE inhibitors and ARBs [27, 115, 116]. However, it remains to be investigated whether metabolic effects would be more pronounced after a more prolonged treatment period. Furthermore, the persistence and durability of the metabolic effects after cessation of treatment also remains to be established.

3. It remains to be established whether sacubitril/valsartan has effects on other key metabolic organs (i.e. visceral adipose tissue, the liver and skeletal muscle), which may underlie the observed improvement in peripheral insulin sensitivity.

4. Future studies should investigate the exercise-mediated metabolic responses in females, since there is a sex difference in metabolic regulation [117119], related to circulating sex hormones [120, 121]. Interestingly, there is a sex difference with respect to circulating NP concentrations [122], and estrogen administration in postmenopausal women increased circulating ANP concentrations [123]. The sex dependent NP regulation might contribute to the well-known differences in cardiovascular risk between men and women. Clearly, 
results obtained in a male population cannot simply be extrapolated to the female population.

5. There is a large inter-individual variation in several metabolic health outcomes following different interventions, including exercise training [124]. Metabolic phenotyping at baseline makes it possible to stratify subjects into different subgroups and may improve the effectiveness of a particular intervention in a specific subgroup of the population [125]. However, we do not find clear evidence that obese individuals with a different metabolic phenotype at baseline, differentially affected exercise training-induced study outcomes. Thus, based on the findings described in this thesis, we cannot conclude that a subgroup-based approach is more beneficial than a population-based approach. Therefore, studies including more detailed metabolic phenotyping such as tissue-specific profiling are needed not only to identify individuals or subgroups at increased risk of developing metabolic diseases but also to design optimized prevention and treatment strategies for specific subgroups of the population, and ultimately leading to personalized exercise strategies. 


\section{REFERENCES}

1. Despres JP, Lemieux I. Abdominal obesity and metabolic syndrome. Nature. 2006;444(7121):881-7.

2. Kahn SE, Hull RL, Utzschneider KM. Mechanisms linking obesity to insulin resistance and type 2 diabetes. Nature. 2006;444(7121):840-6.

3. Van Gaal LF, Mertens IL, De Block CE. Mechanisms linking obesity with cardiovascular disease. Nature. 2006;444(7121):875-80.

4. Goossens GH. The role of adipose tissue dysfunction in the pathogenesis of obesityrelated insulin resistance. Physiol Behav. 2008;94(2):206-18.

5. Rosen ED, Spiegelman BM. What we talk about when we talk about fat. Cell. 2014;156(1-2):20-44.

6. Goossens $\mathrm{GH}$. The renin-angiotensin system in the pathophysiology of type 2 diabetes. Obes Facts. 2012;5(4):611-24.

7. Frigolet ME, Torres N, Tovar AR. The renin-angiotensin system in adipose tissue and its metabolic consequences during obesity. J Nutr Biochem. 2013;24(12):2003-15.

8. Ramalingam L, Menikdiwela K, LeMieux M, Dufour JM, Kaur G, Kalupahana N, et al. The renin angiotensin system, oxidative stress and mitochondrial function in obesity and insulin resistance. Biochim Biophys Acta. 2016.

9. Borghi F, Seva-Pessoa B, Grassi-Kassisse DM. The adipose tissue and the involvement of the renin-angiotensin-aldosterone system in cardiometabolic syndrome. Cell Tissue Res. 2016;366(3):543-8.

10. Moro C. Targeting cardiac natriuretic peptides in the therapy of diabetes and obesity. Expert Opin Ther Targets. 2016;20(12):1445-52.

11. Goossens GH, Blaak EE, van Baak MA. Possible involvement of the adipose tissue renin-angiotensin system in the pathophysiology of obesity and obesity-related disorders. Obes Rev. 2003;4(1):43-55.

12. Unger $\mathrm{T}$. The role of the renin-angiotensin system in the development of cardiovascular disease. Am J Cardiol. 2002;89(2A):3A-9A; discussion 10A.

13. Wang TJ, Larson MG, Levy D, Benjamin EJ, Leip EP, Wilson PW, et al. Impact of obesity on plasma natriuretic peptide levels. Circulation. 2004;109(5):594-600.

14. Das SR, Drazner MH, Dries DL, Vega GL, Stanek HG, Abdullah SM, et al. Impact of body mass and body composition on circulating levels of natriuretic peptides: results from the Dallas Heart Study. Circulation. 2005;112(14):2163-8.

15. Khan AM, Cheng S, Magnusson M, Larson MG, Newton-Cheh C, McCabe EL, et al. Cardiac natriuretic peptides, obesity, and insulin resistance: evidence from two community-based studies. J Clin Endocrinol Metab. 2011;96(10):3242-9.

16. Langenickel T.H. DWP. Angiotensin receptor-neprilysin inhibition with LCZ696: a novel approach for the treatment of heart failure. Drug Discovery Today: Therapeutic Strategies. 2012;9(4):e131-e9.

17. Tschop MH, Finan B, Clemmensen C, Gelfanov V, Perez-Tilve D, Muller TD, et al. Unimolecular Polypharmacy for Treatment of Diabetes and Obesity. Cell Metab. 2016;24(1):51-62.

18. Levin PA. Practical combination therapy based on pathophysiology of type 2 diabetes. Diabetes Metab Syndr Obes. 2016;9:355-69.

19. McMurray JJ, Packer M, Desai AS, Gong J, Lefkowitz MP, Rizkala AR, et al. Dual angiotensin receptor and neprilysin inhibition as an alternative to angiotensinconverting enzyme inhibition in patients with chronic systolic heart failure: rationale for and design of the Prospective comparison of ARNI with ACEI to Determine Impact on Global Mortality and morbidity in Heart Failure trial (PARADIGM-HF). Eur J Heart Fail. 2013;15(9):1062-73. 
20. Tuomilehto J, Lindstrom J, Eriksson JG, Valle TT, Hamalainen H, Ilanne-Parikka P, et al. Prevention of type 2 diabetes mellitus by changes in lifestyle among subjects with impaired glucose tolerance. N Engl J Med. 2001;344(18):1343-50.

21. Corpeleijn E, Feskens EJ, Jansen EH, Mensink M, Saris WH, de Bruin TW, et al. Improvements in glucose tolerance and insulin sensitivity after lifestyle intervention are related to changes in serum fatty acid profile and desaturase activities: the SLIM study. Diabetologia. 2006;49(10):2392-401.

22. Lin X, Zhang X, Guo J, Roberts CK, McKenzie S, Wu WC, et al. Effects of Exercise Training on Cardiorespiratory Fitness and Biomarkers of Cardiometabolic Health: A Systematic Review and Meta-Analysis of Randomized Controlled Trials. J Am Heart Assoc. 2015;4(7).

23. Cassidy S, Thoma C, Houghton D, Trenell MI. High-intensity interval training: a review of its impact on glucose control and cardiometabolic health. Diabetologia. 2017;60(1):7-23.

24. Stanford KI, Middelbeek RJ, Goodyear LJ. Exercise Effects on White Adipose Tissue: Beiging and Metabolic Adaptations. Diabetes. 2015;64(7):2361-8.

25. Thompson D, Karpe F, Lafontan M, Frayn K. Physical activity and exercise in the regulation of human adipose tissue physiology. Physiol Rev. 2012;92(1):157-91.

26. Stanford KI, Goodyear LJ. Exercise regulation of adipose tissue. Adipocyte. 2016;5(2):153-62.

27. van der Zijl NJ, Moors CC, Goossens GH, Hermans MM, Blaak EE, Diamant M. Valsartan improves \{beta\}-cell function and insulin sensitivity in subjects with impaired glucose metabolism: a randomized controlled trial. Diabetes Care. 2011;34(4):845-51.

28. Top C, Cingozbay BY, Terekeci H, Kucukardali Y, Onde ME, Danaci M. The effects of valsartan on insulin sensitivity in patients with primary hypertension. J Int Med Res. 2002;30(1):15-20.

29. Fogari R, Derosa G, Zoppi A, Rinaldi A, Lazzari P, Fogari E, et al. Comparison of the effects of valsartan and felodipine on plasma leptin and insulin sensitivity in hypertensive obese patients. Hypertens Res. 2005;28(3):209-14.

30. Brook RD, Bard RL, Kehrer C, Bodary PF, Eitzman DT, Rajagopalan S. Valsartan Improves Insulin Sensitivity without Altering Vascular Function in Healthy Overweight Adults without the Metabolic Syndrome. Metab Syndr Relat Disord. 2007;5(3):255-61.

31. Pscherer S, Heemann U, Frank H. Effect of Renin-Angiotensin system blockade on insulin resistance and inflammatory parameters in patients with impaired glucose tolerance. Diabetes Care. 2010;33(4):914-9.

32. Ichikawa Y. Comparative effects of telmisartan and valsartan on insulin resistance in hypertensive patients with metabolic syndrome. Intern Med. 2007;46(17):1331-6.

33. Hill JO, Wyatt HR, Peters JC. Energy balance and obesity. Circulation. 2012;126(1):126-32.

34. Turner N, Cooney GJ, Kraegen EW, Bruce CR. Fatty acid metabolism, energy expenditure and insulin resistance in muscle. J Endocrinol. 2014;220(2):T61-79.

35. Sengenes C, Berlan M, De Glisezinski I, Lafontan M, Galitzky J. Natriuretic peptides: a new lipolytic pathway in human adipocytes. FASEB J. 2000;14(10):1345-51.

36. Birkenfeld AL, Boschmann M, Moro C, Adams F, Heusser K, Franke G, et al. Lipid mobilization with physiological atrial natriuretic peptide concentrations in humans. $J$ Clin Endocrinol Metab. 2005;90(6):3622-8.

37. Birkenfeld AL, Boschmann M, Moro C, Adams F, Heusser K, Tank J, et al. Betaadrenergic and atrial natriuretic peptide interactions on human cardiovascular and metabolic regulation. J Clin Endocrinol Metab. 2006;91(12):5069-75.

38. Moro C, Polak J, Hejnova J, Klimcakova E, Crampes F, Stich V, et al. Atrial natriuretic peptide stimulates lipid mobilization during repeated bouts of endurance exercise. Am J Physiol Endocrinol Metab. 2006;290(5):E864-9. 
39. Moro C, Pillard F, de Glisezinski I, Klimcakova E, Crampes F, Thalamas C, et al. Exercise-induced lipid mobilization in subcutaneous adipose tissue is mainly related to natriuretic peptides in overweight men. Am J Physiol Endocrinol Metab. 2008;295(2):E505-13.

40. Birkenfeld AL, Budziarek P, Boschmann M, Moro C, Adams F, Franke G, et al. Atrial natriuretic peptide induces postprandial lipid oxidation in humans. Diabetes. 2008;57(12):3199-204.

41. Bordicchia M, Liu D, Amri EZ, Ailhaud G, Dessi-Fulgheri P, Zhang C, et al. Cardiac natriuretic peptides act via p38 MAPK to induce the brown fat thermogenic program in mouse and human adipocytes. J Clin Invest. 2012;122(3):1022-36.

42. Souza SC, Chau MD, Yang Q, Gauthier MS, Clairmont KB, Wu Z, et al. Atrial natriuretic peptide regulates lipid mobilization and oxygen consumption in human adipocytes by activating AMPK. Biochem Biophys Res Commun. 2011;410(3):398403.

43. Engeli S, Birkenfeld AL, Badin PM, Bourlier V, Louche K, Viguerie N, et al. Natriuretic peptides enhance the oxidative capacity of human skeletal muscle. J Clin Invest. 2012;122(12):4675-9.

44. Goossens GH, Moors CC, van der Zijl NJ, Venteclef N, Alili R, Jocken JW, et al. Valsartan improves adipose tissue function in humans with impaired glucose metabolism: a randomized placebo-controlled double-blind trial. PLoS One. 2012;7(6):e39930.

45. Chatzinikolaou A, Fatouros I, Petridou A, Jamurtas A, Avloniti A, Douroudos I, et al. Adipose tissue lipolysis is upregulated in lean and obese men during acute resistance exercise. Diabetes Care. 2008;31(7):1397-9.

46. Hansen D, Meeusen R, Mullens A, Dendale P. Effect of acute endurance and resistance exercise on endocrine hormones directly related to lipolysis and skeletal muscle protein synthesis in adult individuals with obesity. Sports Med. 2012;42(5):415-31.

47. Wang TJ, Larson MG, Keyes MJ, Levy D, Benjamin EJ, Vasan RS. Association of plasma natriuretic peptide levels with metabolic risk factors in ambulatory individuals. Circulation. 2007;115(11):1345-53.

48. Joyner MJ, Green DJ. Exercise protects the cardiovascular system: effects beyond traditional risk factors. J Physiol. 2009;587(Pt 23):5551-8.

49. Goodyear LJ, Kahn BB. Exercise, glucose transport, and insulin sensitivity. Annu Rev Med. 1998;49:235-61.

50. Malin SK, Gerber R, Chipkin SR, Braun B. Independent and combined effects of exercise training and metformin on insulin sensitivity in individuals with prediabetes. Diabetes Care. 2012;35(1):131-6.

51. Malin SK, Haus JM, Solomon TP, Blaszczak A, Kashyap SR, Kirwan JP. Insulin sensitivity and metabolic flexibility following exercise training among different obese insulin-resistant phenotypes. Am J Physiol Endocrinol Metab. 2013;305(10):E1292-8.

52. Srikanthan $P$, Karlamangla AS. Relative muscle mass is inversely associated with insulin resistance and prediabetes. Findings from the third National Health and Nutrition Examination Survey. J Clin Endocrinol Metab. 2011;96(9):2898-903.

53. Roberts CK, Little JP, Thyfault JP. Modification of insulin sensitivity and glycemic control by activity and exercise. Med Sci Sports Exerc. 2013;45(10):1868-77.

54. Croymans DM, Paparisto E, Lee MM, Brandt N, Le BK, Lohan D, et al. Resistance training improves indices of muscle insulin sensitivity and beta-cell function in overweight/obese, sedentary young men. J Appl Physiol (1985). 2013;115(9):124553.

55. Prior SJ, Goldberg AP, Ortmeyer HK, Chin ER, Chen D, Blumenthal JB, et al. Increased Skeletal Muscle Capillarization Independently Enhances Insulin Sensitivity 
in Older Adults After Exercise Training and Detraining. Diabetes. 2015;64(10):338695.

56. Gysel T, Tonoli C, Pardaens S, Cambier D, Kaufman JM, Zmierczak HG, et al. Lower insulin sensitivity is related to lower relative muscle cross-sectional area, lower muscle density and lower handgrip force in young and middle aged non-diabetic men. J Musculoskelet Neuronal Interact. 2016;16(4):302-9.

57. Dirks ML, Wall BT, van de Valk B, Holloway TM, Holloway GP, Chabowski A, et al. One Week of Bed Rest Leads to Substantial Muscle Atrophy and Induces WholeBody Insulin Resistance in the Absence of Skeletal Muscle Lipid Accumulation. Diabetes. 2016;65(10):2862-75.

58. Thyfault JP, Krogh-Madsen R. Metabolic disruptions induced by reduced ambulatory activity in free-living humans. J Appl Physiol (1985). 2011;111(4):1218-24.

59. da Luz G, Frederico MJ, da Silva S, Vitto MF, Cesconetto PA, de Pinho RA, et al. Endurance exercise training ameliorates insulin resistance and reticulum stress in adipose and hepatic tissue in obese rats. Eur J Appl Physiol. 2011;111(9):2015-23.

60. Marcinko K, Sikkema SR, Samaan MC, Kemp BE, Fullerton MD, Steinberg GR. High intensity interval training improves liver and adipose tissue insulin sensitivity. Mol Metab. 2015;4(12):903-15.

61. Polak J, Moro C, Klimcakova E, Hejnova J, Majercik M, Viguerie N, et al. Dynamic strength training improves insulin sensitivity and functional balance between adrenergic alpha $2 \mathrm{~A}$ and beta pathways in subcutaneous adipose tissue of obese subjects. Diabetologia. 2005;48(12):2631-40.

62. Skurk T, Alberti-Huber C, Herder C, Hauner H. Relationship between adipocyte size and adipokine expression and secretion. J Clin Endocrinol Metab. 2007;92(3):102333.

63. Murphy J, Moullec G, Santosa S. Factors associated with adipocyte size reduction after weight loss interventions for overweight and obesity: a systematic review and meta-regression. Metabolism. 2017;67:31-40.

64. Andersson DP, Eriksson Hogling D, Thorell A, Toft E, Qvisth V, Naslund E, et al. Changes in subcutaneous fat cell volume and insulin sensitivity after weight loss. Diabetes Care. 2014;37(7):1831-6.

65. Pasarica M, Tchoukalova YD, Heilbronn LK, Fang X, Albu JB, Kelley DE, et al. Differential effect of weight loss on adipocyte size subfractions in patients with type 2 diabetes. Obesity (Silver Spring). 2009;17(10):1976-8.

66. Salans LB, Knittle JL, Hirsch J. The role of adipose cell size and adipose tissue insulin sensitivity in the carbohydrate intolerance of human obesity. J Clin Invest. 1968;47(1):153-65.

67. Despres JP, Bouchard C, Savard R, Tremblay A, Marcotte M, Theriault G. The effect of a 20-week endurance training program on adipose-tissue morphology and lipolysis in men and women. Metabolism. 1984;33(3):235-9.

68. Albu JB, Heilbronn LK, Kelley DE, Smith SR, Azuma K, Berk ES, et al. Metabolic changes following a 1-year diet and exercise intervention in patients with type 2 diabetes. Diabetes. 2010;59(3):627-33.

69. Mendelson M, Michallet AS, Monneret D, Perrin C, Esteve F, Lombard PR, et al. Impact of exercise training without caloric restriction on inflammation, insulin resistance and visceral fat mass in obese adolescents. Pediatr Obes. 2015;10(4):311-9.

70. You T, Murphy KM, Lyles MF, Demons JL, Lenchik L, Nicklas BJ. Addition of aerobic exercise to dietary weight loss preferentially reduces abdominal adipocyte size. Int $\mathrm{J}$ Obes (Lond). 2006;30(8):1211-6.

71. Lonnqvist F, Nordfors L, Jansson M, Thorne A, Schalling M, Arner P. Leptin secretion from adipose tissue in women. Relationship to plasma levels and gene expression. $J$ Clin Invest. 1997;99(10):2398-404. 
72. Bahceci M, Gokalp D, Bahceci S, Tuzcu A, Atmaca S, Arikan S. The correlation between adiposity and adiponectin, tumor necrosis factor alpha, interleukin-6 and high sensitivity C-reactive protein levels. Is adipocyte size associated with inflammation in adults? J Endocrinol Invest. 2007;30(3):210-4.

73. Sjogren P, Sierra-Johnson J, Kallings LV, Cederholm T, Kolak M, Halldin M, et al. Functional changes in adipose tissue in a randomised controlled trial of physical activity. Lipids Health Dis. 2012;11:80.

74. Trachta P, Drapalova J, Kavalkova P, Touskova V, Cinkajzlova A, Lacinova Z, et al. Three months of regular aerobic exercise in patients with obesity improve systemic subclinical inflammation without major influence on blood pressure and endocrine production of subcutaneous fat. Physiol Res. 2014;63 Suppl 2:S299-308.

75. Klimcakova E, Polak J, Moro C, Hejnova J, Majercik M, Viguerie N, et al. Dynamic strength training improves insulin sensitivity without altering plasma levels and gene expression of adipokines in subcutaneous adipose tissue in obese men. $\mathrm{J}$ Clin Endocrinol Metab. 2006;91(12):5107-12.

76. Polak J, Klimcakova E, Moro C, Viguerie N, Berlan M, Hejnova J, et al. Effect of aerobic training on plasma levels and subcutaneous abdominal adipose tissue gene expression of adiponectin, leptin, interleukin 6 , and tumor necrosis factor alpha in obese women. Metabolism. 2006;55(10):1375-81.

77. Christiansen T, Paulsen SK, Bruun JM, Pedersen SB, Richelsen B. Exercise training versus diet-induced weight-loss on metabolic risk factors and inflammatory markers in obese subjects: a 12-week randomized intervention study. Am J Physiol Endocrinol Metab. 2010;298(4):E824-31.

78. Alvehus M, Boman N, Soderlund K, Svensson MB, Buren J. Metabolic adaptations in skeletal muscle, adipose tissue, and whole-body oxidative capacity in response to resistance training. Eur J Appl Physiol. 2014;114(7):1463-71.

79. Chen L, Na R, Gu M, Salmon AB, Liu Y, Liang H, et al. Reduction of mitochondrial $\mathrm{H} 2 \mathrm{O} 2$ by overexpressing peroxiredoxin 3 improves glucose tolerance in mice. Aging Cell. 2008;7(6):866-78.

80. Kusminski CM, Scherer PE. Mitochondrial dysfunction in white adipose tissue. Trends Endocrinol Metab. 2012;23(9):435-43.

81. Yoneshiro T, Aita S, Matsushita M, Kayahara T, Kameya T, Kawai Y, et al. Recruited brown adipose tissue as an antiobesity agent in humans. $J$ Clin Invest. 2013;123(8):3404-8.

82. van der Lans AA, Hoeks J, Brans B, Vijgen GH, Visser MG, Vosselman MJ, et al. Cold acclimation recruits human brown fat and increases nonshivering thermogenesis. J Clin Invest. 2013;123(8):3395-403.

83. Chondronikola M, Volpi E, Borsheim E, Porter C, Annamalai P, Enerback S, et al. Brown adipose tissue improves whole-body glucose homeostasis and insulin sensitivity in humans. Diabetes. 2014;63(12):4089-99.

84. Camera DM, Anderson MJ, Hawley JA, Carey AL. Short-term endurance training does not alter the oxidative capacity of human subcutaneous adipose tissue. Eur $\mathrm{J}$ Appl Physiol. 2010;109(2):307-16.

85. Norheim F, Langleite TM, Hjorth M, Holen T, Kielland A, Stadheim HK, et al. The effects of acute and chronic exercise on PGC-1alpha, irisin and browning of subcutaneous adipose tissue in humans. FEBS J. 2014;281(3):739-49.

86. Ronn T, Volkov P, Tornberg A, Elgzyri T, Hansson O, Eriksson KF, et al. Extensive changes in the transcriptional profile of human adipose tissue including genes involved in oxidative phosphorylation after a 6-month exercise intervention. Acta Physiol (Oxf). 2014;211(1):188-200.

87. Bostrom P, Wu J, Jedrychowski MP, Korde A, Ye L, Lo JC, et al. A PGC1-alphadependent myokine that drives brown-fat-like development of white fat and thermogenesis. Nature. 2012;481(7382):463-8. 
88. Larsen S, Danielsen JH, Sondergard SD, Sogaard D, Vigelsoe A, Dybboe R, et al. The effect of high-intensity training on mitochondrial fat oxidation in skeletal muscle and subcutaneous adipose tissue. Scand J Med Sci Sports. 2015;25(1):e59-69.

89. Ruschke K, Fishbein L, Dietrich A, Kloting N, Tonjes A, Oberbach A, et al. Gene expression of PPARgamma and PGC-1alpha in human omental and subcutaneous adipose tissues is related to insulin resistance markers and mediates beneficial effects of physical training. Eur J Endocrinol. 2010;162(3):515-23.

90. Khadir A, Tiss A, Abubaker J, Abu-Farha M, Al-Khairi I, Cherian P, et al. MAP kinase phosphatase DUSP1 is overexpressed in obese humans and modulated by physical exercise. Am J Physiol Endocrinol Metab. 2015;308(1):E71-83.

91. Morigny $\mathrm{P}$, Houssier $\mathrm{M}$, Mouisel E, Langin D. Adipocyte lipolysis and insulin resistance. Biochimie. 2016;125:259-66.

92. De Glisezinski I, Crampes F, Harant I, Berlan M, Hejnova J, Langin D, et al. Endurance training changes in lipolytic responsiveness of obese adipose tissue. Am J Physiol. 1998;275(6 Pt 1):E951-6.

93. Reynisdottir S, Langin D, Carlstrom K, Holm C, Rossner S, Arner P. Effects of weight reduction on the regulation of lipolysis in adipocytes of women with upper-body obesity. Clin Sci (Lond). 1995;89(4):421-9.

94. Ryden M, Jocken J, van Harmelen V, Dicker A, Hoffstedt J, Wiren M, et al. Comparative studies of the role of hormone-sensitive lipase and adipose triglyceride lipase in human fat cell lipolysis. Am J Physiol Endocrinol Metab. 2007;292(6):E184755.

95. Jocken JW, Goossens GH, van Hees AM, Frayn KN, van Baak M, Stegen J, et al. Effect of beta-adrenergic stimulation on whole-body and abdominal subcutaneous adipose tissue lipolysis in lean and obese men. Diabetologia. 2008;51(2):320-7.

96. Lafontan M, Berlan M. Fat cell adrenergic receptors and the control of white and brown fat cell function. J Lipid Res. 1993;34(7):1057-91.

97. Follenius $\mathrm{M}$, Brandenberger $\mathrm{G}$. Increase in atrial natriuretic peptide in response to physical exercise. Eur J Appl Physiol Occup Physiol. 1988;57(2):159-62.

98. Verboven K, Hansen D, Moro C, Eijnde BO, Hoebers N, Knol J, et al. Attenuated atrial natriuretic peptide-mediated lipolysis in subcutaneous adipocytes of obese type 2 diabetic men. Clin Sci (Lond). 2016;130(13):1105-14.

99. Ryden M, Backdahl J, Petrus P, Thorell A, Gao H, Coue M, et al. Impaired atrial natriuretic peptide-mediated lipolysis in obesity. Int J Obes (Lond). 2016;40(4):71420.

100. Stich V, de Glisezinski I, Crampes F, Suljkovicova H, Galitzky J, Riviere D, et al. Activation of antilipolytic alpha(2)-adrenergic receptors by epinephrine during exercise in human adipose tissue. Am J Physiol. 1999;277(4 Pt 2):R1076-83.

101. Stich V, de Glisezinski I, Galitzky J, Hejnova J, Crampes F, Riviere D, et al. Endurance training increases the beta-adrenergic lipolytic response in subcutaneous adipose tissue in obese subjects. Int J Obes Relat Metab Disord. 1999;23(4):374-81.

102. Moro C, Crampes F, Sengenes C, De Glisezinski I, Galitzky J, Thalamas C, et al. Atrial natriuretic peptide contributes to physiological control of lipid mobilization in humans. FASEB J. 2004;18(7):908-10.

103. Moro C, Pillard F, De Glisezinski I, Harant I, Rivi??Re D, Stich V, et al. Training Enhances ANP Lipid-Mobilizing Action in Adipose Tissue of Overweight Men. Medicine \& Science in Sports \& Exercise. 2005;37(7):1126-32.

104. Bulow J. Human adipose tissue blood flow during prolonged exercise, III. Effect of beta-adrenergic blockade, nicotinic acid and glucose infusion. Scand J Clin Lab Invest. 1981;41(4):415-24.

105. Stich V, De Glisezinski I, Crampes F, Hejnova J, Cottet-Emard JM, Galitzky J, et al. Activation of alpha(2)-adrenergic receptors impairs exercise-induced lipolysis in 
SCAT of obese subjects. Am J Physiol Regul Integr Comp Physiol. 2000;279(2):R499-504

106. Ardilouze JL, Karpe F, Currie JM, Frayn KN, Fielding BA. Subcutaneous adipose tissue blood flow varies between superior and inferior levels of the anterior abdominal wall. Int J Obes Relat Metab Disord. 2004;28(2):228-33.

107. Reynisdottir S, Wahrenberg H, Carlstrom K, Rossner S, Arner P. Catecholamine resistance in fat cells of women with upper-body obesity due to decreased expression of beta 2-adrenoceptors. Diabetologia. 1994;37(4):428-35.

108. Mauriege P, Despres JP, Prud'homme D, Pouliot MC, Marcotte M, Tremblay A, et al. Regional variation in adipose tissue lipolysis in lean and obese men. J Lipid Res. 1991;32(10):1625-33.

109. Zouhal H, Lemoine-Morel S, Mathieu ME, Casazza GA, Jabbour G. Catecholamines and obesity: effects of exercise and training. Sports Med. 2013;43(7):591-600.

110. Zhang J, Hupfeld CJ, Taylor SS, Olefsky JM, Tsien RY. Insulin disrupts betaadrenergic signalling to protein kinase A in adipocytes. Nature. 2005;437(7058):56973.

111. Ahmed K, Tunaru S, Tang C, Muller M, Gille A, Sassmann A, et al. An autocrine lactate loop mediates insulin-dependent inhibition of lipolysis through GPR81. Cell Metab. 2010;11(4):311-9.

112. Liu C, Wu J, Zhu J, Kuei C, Yu J, Shelton J, et al. Lactate inhibits lipolysis in fat cells through activation of an orphan G-protein-coupled receptor, GPR81. J Biol Chem. 2009;284(5):2811-22.

113. Richterova B, Stich V, Moro C, Polak J, Klimcakova E, Majercik M, et al. Effect of endurance training on adrenergic control of lipolysis in adipose tissue of obese women. J Clin Endocrinol Metab. 2004;89(3):1325-31.

114. Moro C, Pasarica M, Elkind-Hirsch K, Redman LM. Aerobic exercise training improves atrial natriuretic peptide and catecholamine-mediated lipolysis in obese women with polycystic ovary syndrome. J Clin Endocrinol Metab. 2009;94(7):257986.

115. Aksnes TA, Reims HM, Guptha S, Moan A, Os I, Kjeldsen SE. Improved insulin sensitivity with the angiotensin II-receptor blocker losartan in patients with hypertension and other cardiovascular risk factors. $J$ Hum Hypertens. 2006;20(11):860-6.

116. Bahr IN, Tretter P, Kruger J, Stark RG, Schimkus J, Unger T, et al. High-dose treatment with telmisartan induces monocytic peroxisome proliferator-activated receptor-gamma target genes in patients with the metabolic syndrome. Hypertension. $2011 ; 58(4): 725-32$.

117. Cheneviere X, Borrani F, Sangsue D, Gojanovic B, Malatesta D. Gender differences in whole-body fat oxidation kinetics during exercise. Appl Physiol Nutr Metab. $2011 ; 36(1): 88-95$.

118. Lundsgaard AM, Kiens B. Gender differences in skeletal muscle substrate metabolism - molecular mechanisms and insulin sensitivity. Front Endocrinol (Lausanne). 2014;5:195.

119. Varlamov O, Bethea CL, Roberts CT, Jr. Sex-specific differences in lipid and glucose metabolism. Front Endocrinol (Lausanne). 2014;5:241.

120. Tarnopolsky MA. Sex differences in exercise metabolism and the role of 17-beta estradiol. Med Sci Sports Exerc. 2008;40(4):648-54.

121. Isacco L, Duche P, Boisseau N. Influence of hormonal status on substrate utilization at rest and during exercise in the female population. Sports Med. 2012;42(4):327-42.

122. Schlueter N, de Sterke A, Willmes DM, Spranger J, Jordan J, Birkenfeld AL. Metabolic actions of natriuretic peptides and therapeutic potential in the metabolic syndrome. Pharmacol Ther. 2014;144(1):12-27. 
123. Maffei S, Del Ry S, Prontera C, Clerico A. Increase in circulating levels of cardiac natriuretic peptides after hormone replacement therapy in postmenopausal women. Clin Sci (Lond). 2001;101(5):447-53.

124. Bohm A, Weigert C, Staiger H, Haring HU. Exercise and diabetes: relevance and causes for response variability. Endocrine. 2016;51(3):390-401.

125. Stefan N, Fritsche A, Schick F, Haring HU. Phenotypes of prediabetes and stratification of cardiometabolic risk. Lancet Diabetes Endocrinol. 2016;4(9):789-98. 
CHAPTER 9 
ADDENDA 


\section{SUMMARY}


Obesity is associated with an increased risk for metabolic impairments and chronic diseases, including insulin resistance, type 2 diabetes and cardiovascular diseases. Strategies to reduce body weight and obesity-related comorbidities include dietary (as discussed in Chapter 2), pharmacological and physical activity interventions. This thesis describes the effects of a pharmacological intervention as well as physical exercise interventions to improve metabolic health in obese individuals, with a focus on adipose tissue metabolism.

An increased renin-angiotensin system activity and a lower activity of the natriuretic peptide system have been linked to the development of type 2 diabetes and cardiovascular disease. Combination therapy with sacubitril/valsartan, a combined angiotensin receptor blocker (ARB) and neprilysin (NEP) inhibitor, facilitates the beneficial effects of the natriuretic peptide system, while inhibiting the detrimental effects of the renin-angiotensin system.

In Chapter 3, we performed a multi-centre, randomized, double-blind, doubledummy, parallel-group study to assess the effects of 8 weeks treatment with sacubitril/valsartan as compared to amlodipine on whole-body insulin sensitivity, determined by a hyperinsulinemic-euglycemic clamp in 98 obese hypertensive patients. We found that sacubitril/valsartan significantly improved peripheral insulin sensitivity without affecting body weight or waist circumference. Furthermore, abdominal subcutaneous adipose tissue lipolysis at rest was slightly but significantly increased in the sacubitril/valsartan as compared to the amlodipine group. Surprisingly, the increased subcutaneous adipose tissue lipolysis at rest did not translate into significant changes in whole-body lipolysis, as measured using the stable isotope $\left[1,1,2,3,3-{ }^{2} \mathrm{H}\right]$-glycerol. Moreover, we also found no significant changes in total energy expenditure and substrate oxidation in resting conditions.

In Chapter 4, we extended the outcome of this multi-centre trial by investigating the effects of sacubitril/valsartan on abdominal subcutaneous adipose tissue and whole-body lipolysis as well as energy metabolism and substrate oxidation during a single bout of moderate-intensity aerobic exercise, which is known to stimulate lipolysis. We observed no significant effects of sacubitril/valsartan as compared to amlodipine on abdominal subcutaneous adipose tissue lipolysis, whole-body lipolysis, energy expenditure and substrate oxidation. Therefore, it seems that sacubitril/valsartan had no physiological relevant effects on adipose tissue lipolysis, whole-body lipolysis and substrate utilization.

To obtain more detailed insight into possible mechanisms underlying the findings described in Chapters 3 and 4, we assessed the effects of sacubitril/valsartan on abdominal subcutaneous adipose tissue gene expression patterns using microarray analysis and determined adipose tissue protein expression profiles in Chapter 5. We showed no significant changes in expression of genes and proteins of factors involved in lipolysis, natriuretic peptide signalling and mitochondrial oxidative metabolism.

Collectively, these data indicate that alterations in abdominal subcutaneous adipose tissue lipolysis, whole-body lipolysis or whole-body substrate oxidation at rest and during exercise, do not seem to contribute to the sacubitril/valsartan- 
induced improvement in peripheral insulin sensitivity. It remains to be established whether sacubitril/valsartan has effects on other key metabolic organs, which may underlie the observed improvement in peripheral insulin sensitivity.

Beside pharmacological therapy, changes in lifestyle are effective in preventing the development of type 2 diabetes and related cardiometabolic complications. There is some evidence, mainly from rodent studies, that exercise training may improve adipose tissue function, thereby reducing obesity-related insulin resistance and other comorbidities. However, human studies that investigated the effects of exercise training on adipose tissue function are limited. Therefore, a second objective of this thesis was to determine the effects of physical exercise training on abdominal subcutaneous adipocyte morphology, adipose tissue gene and protein expression of markers related to adipose tissue function and ex vivo adipocyte lipolysis in metabolically healthy and metabolically compromised individuals.

In Chapter 6, we investigated the effects of a 12-weeks supervised, progressive, combined endurance and resistance exercise training program on insulin sensitivity and adipose tissue function in metabolically healthy and metabolically compromised sedentary, middle-aged men. We found that 12 weeks of exercise training improved body composition, physical fitness and peripheral insulin sensitivity, as assessed by a two-step hyperinsulinemic-euglycemic clamp. However, no significant effects on hepatic and adipose tissue insulin sensitivity, abdominal subcutaneous adipocyte morphology, adipose tissue gene and protein expression of markers related to adipose tissue function, nor $\beta_{2}$-adrenergic sensitivity of abdominal subcutaneous adipose tissue lipolysis were observed in obese subjects, irrespective of their baseline metabolic status. Importantly, since we observed only a slight but significant decrease in fat mass, it is likely that a more pronounced decrease in adipose tissue mass is needed to induce significant changes in adipocyte morphology and, consequently, adipose tissue metabolism.

Also, since atrial natriuretic peptide (ANP) increases during exercise and plays an important role in adipose tissue lipolysis, we investigated abdominal subcutaneous adipose tissue (non-)adrenergically-mediated lipolysis before, during and after a single bout of endurance exercise and after 12-weeks of exercise training in metabolically healthy and metabolically compromised individuals in Chapter 7. Therefore, we investigated the effect of local combined $\alpha$ - and $\beta$-adrenoceptor blockade on local subcutaneous adipose tissue lipolysis at rest, during low-intensity endurance-type exercise and during recovery from exercise in sedentary, middleaged obese insulin sensitive, obese insulin resistant and age-matched lean insulin sensitive men. In addition, we investigated whether a 12-week supervised, progressive, combined endurance and resistance exercise training improved the metabolic profile in obese men and (non-)adrenergically-mediated abdominal subcutaneous adipose tissue lipolysis in obese insulin resistant individuals. We demonstrated a major contribution of non-adrenergically-mediated lipolysis during exercise in all groups. Furthermore, we showed that the exercise training intervention improved body composition, physical fitness and exercise-induced changes in circulating free fatty acids, lactate and adrenalin concentrations in both obese groups and insulin sensitivity in the obese insulin resistant group. However, 
this was not accompanied by changes in adrenergically- and non-adrenergicallymediated lipolysis in the subcutaneous adipose tissue of obese insulin resistant individuals. Together, these data suggest that even after a substantial improvement in metabolic profile and body composition after a 12-week exercise intervention, lipolytic disturbances remain unaffected in subcutaneous adipose tissue of obese insulin resistant individuals. Optimized therapies are warranted to achieve enhancements in the regulation of subcutaneous adipose tissue lipolysis, especially in metabolically compromised individuals.

Combined, these data indicate that even after exercise-induced improvements in body composition, physical fitness and peripheral insulin sensitivity, changes in abdominal subcutaneous adipose tissue metabolism and function are lacking and will most likely occur only after a more pronounced decrease in adipose tissue mass. Currently, it remains to be established which exercise training duration and modality is most optimal to induce beneficial effects in abdominal subcutaneous adipose tissue. 


\section{VALORIZATION}




\section{SOCIAL RELEVANCE}

The worldwide prevalence of obesity has increased enormously over the last decades and numbers are still increasing every year. According to the World Health Organization, $13 \%$ of the world's adult population (11\% of men and $15 \%$ of women) was obese in 2014 , while in the same year, obesity affected around $18,5 \%$ of men and $19 \%$ of woman in The Netherlands [1]. If post-2000 trends continue, this global prevalence of obesity is suggested to reach $18 \%$ in men and $21 \%$ in women by 2025. Obesity is associated with an increased risk of developing chronic diseases, including insulin resistance [2], type 2 diabetes [3], cardiovascular diseases [4] and certain types of cancer [5, 6]. To reduce these obesity related comorbidities, nowadays, millions of people are in need of medication, such as glucose-, cholesterol- and/or blood pressure lowering medication, and surgical treatments such as gastric bypass or cardiovascular surgery. Since obesity is a major public health issue and one of the most important risk factors for the development of metabolic diseases, it is clear that the increasing obesity prevalence has major socioeconomic consequences [7]. National and international guidelines recommend changes in modifiable lifestyle characteristics, such as diet and physical activity for both prevention and management of metabolic diseases [8]. While weight loss has been shown to be effective in reducing disease risk, implementation of the recommended lifestyle in the long-term is often hard to maintain by the majority of people. Therefore, to reduce the incidence of obesity and thereby partly improving global health, it is important to obtain better insights in the development and treatment of obesity and related metabolic diseases and to implement new treatment strategies. The results described in this thesis contribute to a better understanding of the role of adipose tissue metabolism in cardiometabolic health and obesity, and provide leads for possible treatment strategies to reduce or prevent obesity and cardiometabolic complications.

\section{TARGET GROUPS}

\section{Scientific community}

The results described in this thesis have and will become available to the scientific community via publication of scientific articles in international peer-reviewed journals. Additionally, results have been presented at (inter)national conferences to scientists as well as physicians, healthcare professionals and dieticians, working in the fields of obesity, diabetes and metabolism.

\section{Industry}

A part of this thesis was accomplished by the close collaboration between academia and industry and research outcomes are of valuable information to the academic community and both the nutritional and the pharmaceutical industry. The industrial partners can translate the research outcomes to develop improved or novel treatment strategies or products that help to prevent or reduce the prevalence of obesity and obesity-related complications. More specific, the nutritional industry can translate the results from chapter 2 in defining new 
nutritional targets. The pharmacological industry can use the results from this thesis to develop and/or implement new or improved pharmacological therapies or to expand the rationale for prescribing certain cardiovascular drugs in metabolically compromised conditions, since in our studies combination therapy with sacubitril/valsartan was shown to improve both cardiovascular and metabolic risk factors.

\section{Health care professionals}

Healthcare professionals (e.g. dieticians, physiotherapists and physicians) play an important role in stimulating a healthy lifestyle among patients and people at increased risk of developing obesity and related complications. On the other hand, physicians prescribe drugs to reverse for example cardiovascular risk (e.g. hypertension) or to improve glucose metabolism and insulin sensitivity. Although the results from this thesis do no provide direct guidelines for healthcare, they provide better insight in metabolic parameters that can be targeted through certain interventions, applied by physicians and other healthcare professionals. Furthermore, this thesis might provide an indication for more targeted prevention programs in high risk groups of the population (i.e. people with both cardiovascular and metabolic risk factors) and it gives more insight in the mechanism of action of a cardiovascular drug (i.e. sacubitril/valsartan) that also has effects on insulin sensitivity and which therefore may alter the rationale for prescription.

\section{ACTIVITIES AND PRODUCTS}

In this thesis, we have put forward fatty acid metabolism-related pathways in several metabolically active organs that can be targeted by dietary interventions, thereby improving whole-body glucose metabolism and insulin sensitivity. The results from the nutritional review can potentially lead to novel functional foods or food supplements (e.g. pre- and probiotics, polyphenols, plant sterols, improved dietary fat quality). However, before these novel products will become available on the market, more scientific research is necessary to confirm mechanisms, safety and health benefits in individuals at increased risk of developing cardiometabolic diseases.

The results from the pharmacological intervention with sacubitril/valsartan, which has recently been approved by the U.S. Food and Drug Administration (FDA) and the European Medicines Agency (EMA) for the treatment of heart failure [9], showed that the combination therapy has a positive effect on cardiovascular and metabolic risk factors. We showed that treatment with sacubitril/valsartan, as compared to the metabolically neutral calcium antagonist amlodipine, improved peripheral insulin sensitivity in obese hypertensive patients. This knowledge will be immediately used by the pharmaceutical industry. Moreover, sacubitril/valsartan may be a promising alternative for the treatment of hypertension in patients who are at increased risk for developing chronic metabolic diseases (e.g. hypertensive individuals with impaired glucose metabolism). 
The exercise training intervention studies showed that exercise training beneficially affects body composition and physical fitness and is effective to improve obesityrelated disturbances like whole-body insulin resistance. Although 12 weeks of exercise training induced a slight but significant reduction in fat mass, no significant changes in abdominal subcutaneous adipocyte morphology, adipose tissue function, and abdominal subcutaneous adipose tissue lipolysis were observed in obese subjects, irrespective of their baseline metabolic status. Furthermore, we showed that 12 weeks of exercise training did not improve disturbances in subcutaneous adipose tissue lipolysis in obese insulin resistant individuals. It seems that a more pronounced decrease in adipose tissue mass is needed to induce significant changes in adipose tissue metabolism. Currently, it remains to be established which exercise training duration and modality is most optimal to induce beneficial effects in abdominal subcutaneous adipose tissue.

\section{INNOVATION \& IMPLEMENTATION}

All results described in this thesis are novel findings and have, partly, been performed at the Department of Human Biology and Movement Sciences of Maastricht University Medical Center+ and in close collaboration with other universities and industrial partners within The Netherlands and Europe, using stateof-the-art methodologies for both in vivo and ex vivo analyses.

An attractive approach, as described in this thesis, is the application of combination therapy, which simultaneously targets more than one biological pathway or mechanism and therefore may be more effective in reducing disease progression because of additional and/or synergistic effects as compared to monotherapies [10] [11]. With respect to the pharmacological treatment with the combination drug, sacubitril/valsartan, this thesis is the first to describe the beneficial metabolic effects (i.e. improved insulin sensitivity) in obese hypertensive patients. However, to implement these results in treatment strategies for the hypertensive population, more research is necessary to unravel the underlying mechanisms in different metabolic tissues (e.g. skeletal muscle) and long-term health outcomes of therapy with the combinational drug sacubitril/valsartan.

The innovative aspect of the exercise training interventions, was the investigation of exercise training-induced effects on abdominal subcutaneous adipose tissue, since these studies have mainly been performed in rodents whereas human studies are scarce. Our results contribute to the knowledge and provide better insight in exercise training-mediated metabolic changes in abdominal subcutaneous adipose tissue.

Furthermore, metabolic phenotyping at baseline makes it possible to stratify subjects into different subgroups and may improve the effectiveness of a particular intervention in a specific subgroup of the population [12]. However, in this thesis we did not find clear evidence that metabolic phenotype at baseline affected exercise training-induced study outcomes. Therefore, before extrapolating our findings to a larger population, more research is necessary in larger study populations following different intervention strategies (e.g. nutritional, pharmacological as well as 
prolonged exercise training interventions). Studies including more detailed metabolic phenotyping such as tissue-specific profiling are needed not only to identify individuals or subgroups at increased risk of developing metabolic diseases, but also to design optimized prevention and treatment strategies for specific subgroups of the population. These promising strategies will be further investigated by the current project team by performing human intervention trials including in vivo and laboratory analyses, performed via state-of-the-art research methodologies. 


\section{REFERENCES}

1. World Health Organization. Fact sheet: Obesity and overweight. Updated June 2016. 2016.

2. Despres JP, Lemieux I. Abdominal obesity and metabolic syndrome. Nature. 2006;444(7121):881-7.

3. Kahn SE, Hull RL, Utzschneider KM. Mechanisms linking obesity to insulin resistance and type 2 diabetes. Nature. 2006;444(7121):840-6.

4. Van Gaal LF, Mertens IL, De Block CE. Mechanisms linking obesity with cardiovascular disease. Nature. 2006;444(7121):875-80.

5. Bhaskaran K, Douglas I, Forbes H, dos-Santos-Silva I, Leon DA, Smeeth L. Bodymass index and risk of 22 specific cancers: a population-based cohort study of 5.24 million UK adults. Lancet. 2014;384(9945):755-65.

6. Lauby-Secretan B, Scoccianti C, Loomis D, Grosse Y, Bianchini F, Straif K, International Agency for Research on Cancer Handbook Working G. Body Fatness and Cancer--Viewpoint of the IARC Working Group. N Engl J Med. 2016;375(8):7948.

7. Di Cesare M, Bentham J, Stevens GA, Zhou B, Danaei G, Lu Y, Bixby H, Cowan MJ, Riley LM, Hajifathalian K, Fortunato L, Taddei C, Bennett JE, Ikeda N, Zhu D, Zimmermann E, J. ZC. Trends in adult body-mass index in 200 countries from 1975 to 2014: a pooled analysis of 1698 population-based measurement studies with 19.2 million participants. Lancet. 2016;387(10026):1377-96.

8. American Diabetes Association. Standards of Medical Care in Diabetes. Diabetes Care. 2016;39(Suppl 1).

9. US FDA. Entresto Prescribing Information 2015 [updated July 7, 2015. Available from:

http://www.accessdata.fda.gov/drugsatfda_docs/nda/2015/207620Orig1s000Lbl.pdf.

10. Tschop MH, Finan B, Clemmensen C, Gelfanov V, Perez-Tilve D, Muller TD, DiMarchi RD. Unimolecular Polypharmacy for Treatment of Diabetes and Obesity. Cell Metab. 2016;24(1):51-62.

11. Levin PA. Practical combination therapy based on pathophysiology of type 2 diabetes. Diabetes Metab Syndr Obes. 2016;9:355-69.

12. Stefan N, Fritsche A, Schick F, Haring HU. Phenotypes of prediabetes and stratification of cardiometabolic risk. Lancet Diabetes Endocrinol. 2016;4(9):789-98. 


\section{ACKNOWLEDGEMENTS}


Promoveren is iets dat je zeker niet alléén kunt doen en ik ben ervan overtuigd dat een goede samenwerking onmisbaar is tijdens het promoveren. Er zijn een heleboel mensen die een belangrijke bijdrage hebben geleverd aan het tot stand komen van deze thesis en ik wil deze mensen dan ook heel graag bedanken.

Allereerst wil ik mijn promotieteam, Prof. Ellen Blaak en Dr. Gijs Goossens bedanken voor het vertrouwen en de kans die ze mij ongeveer 5 jaar geleden hebben gegeven om aan dit promotieonderzoek te starten.

Ellen, bedankt dat je altijd voor me klaar stond wanneer dat nodig was. Dankzij jouw wetenschappelijk inzicht werd altijd de juiste breedte en diepgang aan de manuscripten gegeven. Ik bewonder het enorm dat je, ondanks je drukke agenda, steeds op korte termijn mijn papers na kon kijken en met constructieve commentaar had voorzien.

Gijs, bedankt voor de goede begeleiding. Zowel tijdens het praktisch werk op de testdagen en de planning daarvan als op wetenschappelijk gebied heb ik veel van je geleerd. Ik heb het enorm gewaardeerd dat ik altijd bij je terecht kon wanneer dat nodig was en dat je altijd bereid was om (uitgebreide ;) ) raad te geven. Jouw kritische blik op de manuscripten en de grammaticale aanpassingen (al vond ik die soms wat te uitgebreid :) ) hebben er steeds voor gezorgd dat de manuscripten verbeterden. Ik vond het ook leuk dat we naast het werk gezellige tijden hebben gehad, zoals bijvoorbeeld op de congressen in Barcelona, Götenborg of in Denemarken.

Ellen en Gijs, bedankt voor de zeer prettige samenwerking gedurende de afgelopen jaren!

Next, I would like to thank all members of the thesis assessment committee, Prof. C. Stehouwer, Prof. J. Glatz, Prof. S. Kersten, Prof. L. van Loon and Prof. B. Stallknecht for taking the time and effort to review my thesis and being present at the official dissertation.

I would also like to thank all co-authors for the pleasant cooperation and for the excellent contributions to improve the work that is presented in this thesis.

Zonder toegewijde proefpersonen had deze thesis nooit tot stand kunnen komen. Mijn oprechte dank gaat daarom uit naar alle mensen die hebben deelgenomen aan de experimentele onderzoeken; voor de moeite, flexibiliteit, gemaakte kilometers en het afstaan van bloed, vet- en spierweefsel en vooral heel veel zweet. Ik heb veel plezierige gesprekken en momenten gehad tijdens de verschillende test- en trainingsdagen.

Mijn paranimfen wil ik even extra bedanken!

Max, bijna 4 jaar lang hebben we een kamer gedeeld en ik had me eerlijk gezegd geen betere kamergenoot kunnen wensen! Wanneer er gewerkt moest worden, werd er gewerkt en wanneer we even wouden pauzeren, kon dat gewoon en konden we echt over vanalles praten. We hebben samen veel gelachen en een hele leuke tijd gehad op de summer school in Lissabon. Naast het werk ben je ook een hele gezellige persoon en ik kijk er naar uit om samen nog eens een whisky te gaan proeven ;) 
Birgitta, ook wij hebben een aanzienlijke tijd een kamer gedeeld. We zijn ongeveer tegelijkertijd begonnen op de universiteit en ik heb je leren kennen als een sociale, harde werker die heel direct en eerlijk is. Naast het werk kunnen we het goed met elkaar vinden en hebben we vele leuke gesprekken en tijden gehad, zoals bijvoorbeeld op het congres in Barcelona en op verschillende feestjes.

Max en Birgitta, ik ben blij dat jullie mijn paranimfen willen zijn en het is voor mij een hele geruststelling dat jullie aan mijn zijde staan!

Een groot deel van deze thesis gaat over een farmacologische interventie die synergetische effecten kan hebben. Het is terecht om te zeggen dat ons onderzoeksteam tijdens deze studie ook een synergetisch geheel was ;) Birgitta en Laura, ik wil jullie alle twee heel erg bedanken voor de hulp tijdens deze toch wel stressvolle periode, waarin we soms verschillende screeningen én $\mathrm{VO}_{2} \mathrm{max}$ testen op één ochtend moesten combineren met een testdag. Zonder jullie hulp zou het nooit gelukt zijn om deze studie binnen de deadline tot een goed einde te brengen! Ook Bas, Kirsten, Bart, Irene, Nicolaas en iedereen die op één of andere manier heeft geholpen, bedankt voor de flexibiliteit en bereidwilligheid!

Kenneth, ik wil jou ook even bedanken voor de aangename samenwerking tijdens onze studie in Hasselt. Je hebt enorm veel werk geleverd, hele lange dagen gemaakt (zeker wanneer er zowel in de ochtend als in de avond training sessies plaatsvonden) en heel veel cupjes geplakt ;) Ik ben blij dat we de studie samen tot een goed einde hebben gebracht, dat er mooie data zijn uitgekomen en dat we niet nog één aflevering van "In de gloria" of de film "Intouchable" moeten zien ;)

Ik wens je nog veel succes met het uitbouwen van je verdere academische carrière.

Ik wil ook alle (ex)collega's binnen onze onderzoeksgroep, Johan, Nicole $H$, Yvonne, Jasper, Emanuel, Dorien, Birgitta, Max, Kenneth, Laura, Mattea, Qing, Nicole V, Ruth, Rens, Manuel, Adriyan en Kelly bedanken voor de wetenschappelijke discussies en de leuke tijden die we samen hebben gehad tijdens onze meetings of congressen. De collegialiteit binnen onze onderzoeksgroep vind ik echt super! Wanneer er iemand hulp nodig heeft met testdagen of als iemand met een vraag zit is iedereen wel bereid om te helpen waar hij kan en niets is teveel gevraagd...echt top! Niet alleen professioneel, maar ook naast het werk kunnen we het goed met elkaar vinden. De leuke tijden op congressen, dagjes uit of (trouw)feesten ga ik zeker missen! Ik wil iedereen nog heel veel succes wensen met zijn verdere carrière, maar ik hoop dat we elkaar nog regelmatig kunnen zien. Nicole en Yvonne, bedankt voor de goede begeleiding en de leuke samenwerking op het lab en natuurlijk de leuke praatjes tussendoor!

Alle collega's van Humane Biologie en Bewegingswetenschappen (ik ga bewust geen namen noemen, want het zijn er gewoon te veel en ik wil niemand vergeten), bedankt voor de leuke momenten gedurende de afgelopen 5 jaar! Weekendjes in de Ardennen, dagjes uit, feestjes, de gezellige lunches of diners, de koffiepauzes, kerstdiners en de feestjes die daarop volgden en niet te vergeten de vrijdagmiddag borrels...jullie hebben allemaal bijgedragen aan deze leuke tijden en ik hoop dat de onderlinge sfeer binnen de afdeling zo goed blijt! 
Ik wil alle betrokken analisten bedanken voor het uitvoeren van de vele analyses. Zonder jullie was dit alles nooit gelukt!

De secretaresses wil ik bedanken voor alle ondersteuning en het administratieve werk.

Natuurlijk wil ik ook mijn vrienden bedanken voor de leuke en ontspannende momenten! Het is altijd heel gezellig met jullie en ik hoop dat we nog lang en regelmatig kunnen afspreken, zeker aangezien er nu bouwgronden worden gekocht en over gezinsuitbreiding wordt nagedacht ;)

Mijn familie mag zeker niet ontbreken...Peter en Els (en Amber), Inge en Geert (Stan en Sien), dank jullie voor de interesse, steun en vooral voor de gezellige en leuke momenten die we altijd samen hebben!

Liefste mama en papa, bedankt voor alles wat jullie voor mij gedaan hebben! Bedankt voor jullie onvoorwaardelijke liefde, om altijd voor ons klaar te staan wanneer dat nodig was, voor jullie interesse, raad en steun en dat jullie mij de mogelijkheden hebben gegeven om te komen waar ik nu ben! Ik hoop dat we nog lang in goede gezondheid bij elkaar kunnen zijn! Ik kan jullie niet genoeg bedanken, maar als teken van dank, wil ik deze thesis heel graag aan jullie opdragen! 
CURRICULUM VITAE 
Rudi Stinkens was born on June $2^{\text {nd }} 1983$ in Sittard, The Netherlands and raised in Belgium. He studied Social and Technical Sciences and completed secondary school at the Sint-Augustinus Insituut in Bree, Belgium in 2001. Consecutively, he started a Bachelor of Science in Nutrition and Dietetics at the Katholiek Hogeschool Kempen in Geel, Belgium, where he graduated in 2005. During the following years, he specialised in sports nutrition at both the HAN University of Applied Sciences in Nijmegen, The Netherlands and the Artesis \& Plantijn Hogeschool in Antwerpen, Belgium, while working in the food industry.

In 2010 he decided to make a switch in his career and started the Master of Science program in Physical Activity and Health at Maastricht University, The Netherlands, where he graduated in 2011. During this Master program, he investigated astaxanthin supplementation in endurance trained athletes during a 6months internship at the department of Human Movement Sciences at Maastricht University. Following his internship, he performed several months pro deo research towards beet root juice supplementation, under the supervision of Dr. Cermak N. and Prof. van Loon LJ.

In November 2012, he started as a Ph.D. candidate at the department of Human Biology at Maastricht University (NUTRIM School of Nutrition and Translational Research in Metabolism), under supervision of Prof. Dr. Ellen Blaak and Dr. Gijs Goossens. His research is described in this thesis and is entitled "Adipose tissue metabolism and cardiometabolic health in obesity - Effects of pharmacological and lifestyle interventions". During his Ph.D., Rudi was selected by the European Association for the Study of Obesity (EASO) to attend the $1^{\text {st }}$ Young Investigators United summer school in Lisbon, Portugal (2015). He was also selected as one of the 10 best candidates from the Netherlands to present his research findings at the North European Young Diabetologists (NEYD) meeting in cooperation with the Danish Diabetes Academy in Snekkersten, Denmark (2016). He presented his research findings at several national and international conferences. Furthermore, he received a travel grant from The Netherlands Association for the Study of Obesity (NASO) to present his research at the $1^{\text {st }}$ European Obesity Summit in Göteborg, Sweden (2016) as well as a travel grant from the European Association for the Study of Diabetes (EASD) to present his research at the $53^{\text {rd }}$ Annual Meeting of the European Association for the Study of Diabetes (EASD) in Lisbon, Portugal (2017). 


\section{LIST OF PUBLICATIONS}




\section{FULL PAPERS}

Improved insulin sensitivity with angiotensin receptor neprilysin inhibition in individuals with obesity and hypertension

Jordan J, Stinkens R, Jax T, Engeli S, Blaak EE, May M, Havekes B, Schindler C, Albrecht D, Pal P, Heise T, Goossens GH, Langenickel TH.

Clin Pharmacol Ther. 2017 Feb;101(2):254 - 263

Targeting fatty acid metabolism to improve glucose metabolism

Stinkens R, Goossens GH, Jocken JW, Blaak EE.

Obes Rev. 2015 Sep;16(9):715-757

Astaxanthin supplementation does not augment fat use or improve endurance performance

Res P, Cermak NM, Stinkens R, Tollakson TJ, Haenen GR, Bast A, van Loon LJC.

Med Sci Sports Exerc. 2013 Jun;45(6):1158-65

No improvement in endurance performance after a single dose of beetroot juice Cermak NM, Res P, Stinkens R, Lundberg JO, Gibala MJ, van Loon LJC.

Int J Sport Nutr Exerc Metab. 2012 Dec;22(6):470-8

Effect of sacubitril/valsartan on exercise induced lipid metabolism in individuals with obesity and hypertension

Engeli S, Stinkens R, Heise T, May M, Goossens GH, Blaak EE, Jax T, Albrecht D, Pal P, Tegtber U, Haufe S, Langenickel TH, Jordan J.

Submitted

The effects of angiotensin receptor neprilysin inhibition by sacubitril/valsartan on adipose tissue transcriptome and protein expression in obese hypertensive patients

Stinkens R, van der Kolk BW, Jordan J, Jax T, Engeli S, Heise T, Jocken JW, May M, Schindler C, Havekes B, Schaper N, Albrecht D, Kaiser S, Hartmann N, Letzkus M, Langenickel TH, Goossens GH, Blaak EE.

Submitted

Exercise training-induced effects on abdominal subcutaneous adipose tissue phenotype in obese humans

Stinkens $\mathbf{R}^{*}$, Brouwers $\mathrm{B}^{*}$, Jocken JW, Blaak EE, Theunissen-Beekman KF, Hesselink MK, van Baak M, Schrauwen P, Goossens GH.

To be submitted * $\quad$ Shared first authorship

Coordinated regulation of adipose tissue adrenergic- and non-adrenergic-mediated lipolysis during exercise in lean and obese individuals: the effect of exercise training

Stinkens $\mathbf{R}^{\star}$, Verboven $\mathrm{K}^{*}$, Hansen D, Wens I, Frederix I, Eijnde BO, Jocken JW, Goossens GH", Blaak EE."

Submitted

* Shared first authorship, ${ }^{\#}$ Shared last authorship 


\section{ABSTRACTS}

Coordinated regulation of adipose tissue adrenergic- and non-adrenergic-mediated lipolysis during exercise in lean and obese individuals: the effect of exercise training

Stinkens R, Verboven K, Hansen D, Wens I, Frederix I, Eijnde BO, Jocken JW, Goossens GH, Blaak EE.

Diabetologia, 2017; 60(Suppl.1):1

Exercise training-induced effects on abdominal subcutaneous adipose tissue gene and protein expression and adipose tissue morphology in obese humans

Stinkens R, Brouwers B, Jocken JW, Blaak EE, Hesselink M, Schrauwen P, Goossens GH.

Obesity Facts, 2016; 6(9) (Suppl 1):38

LCZ696 improves lipid mobilization from adipose tissue: A randomized, doubleblind, active-controlled, parallel-group study in obese hypertensive patients

Jordan J, Stinkens R, Jax T, Engeli S, Haufe S, Blaak EE, May M, Havekes B, Schindler C, Albrecht D, Pal P, Schaper N, van der Kolk B, Tegtbur U, Heise T, Goossens GH, Langenickel TH.

Obesity Facts, 2016; 6(9) (Suppl 1):152

LCZ696 improves lipid mobilization from adipose tissue: a randomized, doubleblind, active-controlled, parallel-group study in obese hypertensive patients Jordan J, Stinkens R, Jax T, Engeli S, Haufe S, Blaak EE, May M, Havekes B, Schindler C, Albrecht D, Pal P, Schaper N, van der Kolk B, Tegtbur U, Heise T, Goossens GH, Langenickel TH.

Circulation, 2015; 132 (Supp/ 3):A15144

Metabolic benefits of LCZ696: a randomized, double-blind, active-controlled, parallel-group study in obese hypertensive patients

Jordan J, Stinkens R, Jax T, Engeli S, Blaak EE, May M, Havekes B, Schindler C, Albrecht D, Pal P, Heise T, Goossens GH, Langenickel TH.

Diabetes, 2015; 64 (Suppl 1):A496-A574 